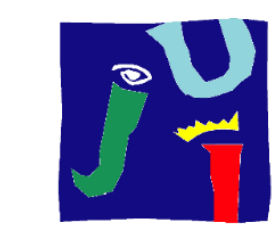

$\mathbf{U}_{\text {NIVERSITAT }}$

JAUME•I

Programa de Doctorado en Ciencias

Escuela de Doctorado de la Universitat Jaume I

\title{
SÍNTESIS Y CARACTERIZACIÓN DE MATERIALES MULTIFUNCIONALES BASADOS EN ÓXIDOS MIXTOS $\mathrm{ZrO}_{2}-\mathrm{Y}_{2} \mathrm{O}_{3}$
}

Memoria presentada por Marc Jovaní Beltran para optar al grado de doctor por la Universitat Jaume I 

Als meus pares

Als meus "abuelos" 



\section{AGRADECIMIENTOS}

Quisiera aprovechar estas líneas para agradecer a todas las personas que de una u otra forma me han ayudado a que la presente tesis sea hoy una realidad.

En primer lugar agradecer a mis directores de tesis, la catedrática Eloisa Cordoncillo Cordoncillo y al doctor Héctor Beltrán Mir, por la oportunidad brindada para realizar esta tesis. Mis palabras de agradecimiento por su ayuda supervisión y dedicación, sin los cuales la realización de la presente tesis hubiera sido imposible.

También mostrar mis agradecimientos al Professsor Anthony R. West por la ayuda prestada. Ha sido un placer poder trabajar y aprender de una persona de su importancia en el campo de la química del estado sólido.

También quisiera agradecer a todos los compañeros/as del grupo de Química del Estado Sólido de la Universitat Jaume I, con los que además de compartir despacho hemos compartido buenos momentos tanto dentro y fuera de la universidad. En especial me gustaría dar las gracias a Maria Domingo ("la ramanera"), Miriam, Alba, Ana, Thales y Maria Fortuño. Pero también a todos los compañeros que han pasado en algún momento del transcurso de la presente tesis por el grupo y con los que he compartido muchos momentos de los cuales guardo muy buen recuerdo.

Quisiera agradecer también a todos los técnicos y compañeros del Serveis Centrals d' Instrumentació Científica (SCIC), por las facilidades proporcionadas para el desarrollo de mi investigación. Además, he tenido el placer de trabajar durante dos años en este centro durante la realización de la presente tesis.

Agradecer a la Universitat Jaume I por la financiación proporcionada por el proyecto P11B2P13-65. 
También me gustaría mencionar a todos mis amigos por el apoyo mostrado, ya que sin ellos la realización de la presente tesis hubiera sido mucho más dificultosa. Sin lugar a duda, las reuniones en "little Xert" después del laboratorio han sido de gran inspiración para la realización de la misma.

Me gustaría hacer mención especial a mis padres, ya que me han apoyado siempre en todo lo que he hecho y sin su ayuda la realización de esta tesis hubiera sido imposible.

Ya por último, y no por ello menos importante, agradecerle a Esther ("la física loca"), por su ayuda y apoyo incondicional, que con su presencia ha hecho este último tramo de tesis mucho más llevadero.

A todos vosotros, muchas gracias. 


\section{Resumen}

Actualmente vivimos en una sociedad desarrollada en la que son muchas las comodidades que nos rodean y nos hacen en cierta forma la vida más fácil. Esta mejora en la calidad de vida en comparación con años atrás, ha venido dada por el desarrollo de la ciencia y por consiguiente, por las innovaciones tecnológicas que esta ha aportado. Es decir, disfrutamos de una calidad de vida envidiable en comparación con nuestros antepasados, gracias en gran parte al gran abanico de mejoras tecnológicas logradas a lo largo de los años. Y siguiendo con esta tendencia, parece ser que dentro de 30 años aún será mejor que la actual, gracias a las mejoras tecnológicas que están por venir.

Sin embargo, estas mejoras derivadas del desarrollo tecnológico, no llevan consigo solamente hechos positivos, ya que indirectamente la mejora de las condiciones de vida conlleva un incremento de la población mundial, un mayor gasto de energía, necesidad de mayor producción de alimentos, contaminación ambiental y otros fenómenos que se han convertido en cierta forma un problema para la humanidad. En consecuencia, parece necesario un desarrollo científico sostenible, que pueda ayudar a resolver muchos de estos problemas o que al menos no los empeore.

El campo en el que se encuentra enmarcada la presente tesis, el de los materiales cerámicos, está en constante desarrollo y es uno de los más prometedores a la hora de minimizar los impactos ambientales, promoviendo por tanto un desarrollo sostenible. Además, las nuevas necesidades sociales que surgen día a día, obligan a la generación constante de un sinfín de nuevos y mejores materiales. Es un hecho probado en la actualidad, la demanda creciente de nuevos materiales mucho más especializados capaces de satisfacer las diversas necesidades surgidas en la sociedad. 
En este sentido, la presente tesis aborda la síntesis, caracterización y aplicación de materiales con estequiometría $\mathrm{A}_{2} \mathrm{~B}_{2} \mathrm{O}_{7}$, a los que pertenece la composición $\mathrm{Y}_{2} \mathrm{Zr}_{2} \mathrm{O}_{7}$, los cuales presentan un gran abanico de aplicaciones en el campo de los materiales cerámicos. Este gran abanico de aplicaciones radica en la posibilidad de la estructura para albergar en su red gran variedad de cationes, confiriéndole una versatilidad de propiedades y por lo tanto, de aplicaciones en diferentes campos, es decir nos encontramos ante una estructura potencialmente multifuncional dependiendo de los iones dopantes que se utilicen.

De esta forma y haciendo uso del sistema $\mathrm{ZrO}_{2}-\mathrm{Y}_{2} \mathrm{O}_{3}$ se ha abordado el estudio de las distintas propiedades ópticas y eléctricas que estas composiciones pueden proporcionar.

Así pues, la presente tesis doctoral se divide en cuatro partes. Una primera parte introductoria, la cual está dedicada al estudio de las características de la propia estructura, las diferentes fases que se pueden formar en función de las composiciones, así como las ventajas que proporciona el método de síntesis utilizado a la hora de estabilizar dichas fases. Adicionalmente se introducirá la problemática existente en la industria de los pigmentos cerámicos, así como algunos conceptos previos y los diferentes antecedentes en materiales similares en el campo de la electrocerámica. Esta parte corresponde al capítulo 1.

Tras plantear los objetivos en el capítulo 2, la segunda parte está dedicada al desarrollo de nuevos pigmentos cerámicos basados en la estructura $\mathrm{Y}_{2} \mathrm{Zr}_{2} \mathrm{O}_{7}$ dopada con diversos iones cromóforos, para su aplicación como pigmento cerámico multifuncional. Esta sección, correspondiente a los capítulos 3 y 4, dio lugar a la publicación de dos artículos científicos en revistas de alto índice de impacto en este campo, como Dyes and Pigments y Journal of the European Ceramic Society.

Una tercera parte, correspondiente al capítulo 5, donde se han estudiado las propiedades eléctricas de compuesto pertenecientes al sistema $\mathrm{ZrO}_{2}-\mathrm{Y}_{2} \mathrm{O}_{3}$ y la 
influencia del itrio en el mecanismo de conducción predominante. Esta sección dio lugar a la publicación de un artículo científico en la revista de alto índice de impacto en este campo como es, Inorganic Chemistry.

Ya para terminar en un último capítulo, el capítulo 6 , se exponen en conjunto las diferentes conclusiones extraídas de los estudios realizados en la presente tesis. 



\section{ABREVIATURAS}

BT Barium Titanate (Óxido mixto de bario y titanio)

CIE Comission Internacionale de 1’Eclairage

XRD X-Ray Diffraction (Difracción de rayos X, DRX)

ECHA European Chemicals Agency (Agencia Europea de Sustancias y Mezclas Químicas)

EDX Energy Dispersive X-Ray (Energías dispersivas de rayos X)

PL Photoluminiscense (Fotoluminiscencia)

ICDD International Centre for Diffraction Data

IE Impedance Spectroscopy (Espectroscopia de impedancia, EI)

JCPPDS Joint Committee on Powder Diffraction Standards

NIR Near Infrared Region (Región Infrarroja Cercana)

PLD Pulsed Laser Deposition (Deposición Pulsada por Laser)

PMMA Poly (methyl methacrylate) (Polimetilmetracrilato)

REACH Registration, Evaluation, Authorization and Restriction (Registro, Evaluación, Autorización y Restricción de sustancias y mezclas químicas)

SEM Scanning Electron Microscopy (Microscopía electrónica de barrido, MEB)

SOFC Solide Oxide Fuell Cells (Celdas de combustible de óxido sólido)

UV/Vis Ultraviolet-Visible (Ultravioleta-visible)

XPS X-Ray Photoelectron Spectroscopy (Espectroscopia Fotoelectrónica de Rayos X)

YSZ Yttria-Stabilized Zirconia (Circona estabilizada con itria)

YSZ08 Circona estabilizada con un $\sim 8 \%$ molar de itria 


$\begin{array}{cl}\mathrm{C} & \text { Capacitancia } \\ \mathrm{c}-\mathrm{ZrO}_{2} & \text { Circona cúbica } \\ d c & \text { Corriente continua } \\ \mathrm{m}-\mathrm{ZrO}_{2} & \text { Circona monoclínica } \\ \mathrm{pO}_{2} & \text { Presión parcial de oxígeno } \\ \mathrm{r} & \text { Radio iónico } \\ \mathrm{R} & \text { Resistencia } \\ \mathrm{t}-\mathrm{ZrO}_{2} & \text { Circona tetragonal }\end{array}$




\section{TABLA DE CONTENIDOS}

CAPÍTULO 1: Aspectos generales 1

1.1.Introducción 3

1.2.La estequiometría $\mathrm{A}_{2} \mathrm{~B}_{2} \mathrm{O}_{7}$

1.3.Diagrama de equilíbrio de fases para el sistema $\mathrm{ZrO}_{2}-\mathrm{Y}_{2} \mathrm{O}_{3} \quad 8$

1.4.Método de síntesis $\quad 19$

1.5.Pigmentos Cerámicos $\quad 24$

1.5.1.Origen del color en sólidos inorgánicos 26

1.5.2.Problemática de los pigmentos cerámicos en la actualidad $\quad 28$

1.5.3.Nuevas aplicaciones de los pigmentos cerámicos 31

1.5.4.La estructura tipo $\mathrm{A}_{2} \mathrm{~B}_{2} \mathrm{O}_{7}$ como red huésped de diferentes 33

cromóforos

1.5.5. $\mathrm{Y}_{2} \mathrm{Zr}_{2} \mathrm{O}_{7}$ como red huésped y sus potenciales aplicaciones 36

1.6.Electroceramica 37

1.6.1.Mecanismo de conducción iónica en YSZ 38

1.6.2.Conducción electrónica en YSZ 41

1.6.3. Mecanismos de conducción electrónica tipo n y p en 42

electrocerámicas. Efecto de la atmósfera

1.6.4.Respuesta de un material cerámico a la aplicación de un voltaje $\quad 46$ externo

\section{CAPÍTULO 2: Objetivos}

\section{CAPÍTULO 3: Síntesis del pigmento multifuncional basado en $\mathrm{Y}_{2} \mathrm{Zr}_{2} \mathrm{O}_{7}$ dopado con $\mathrm{Tb}$ y $\mathrm{Fe}$}


3.1.Introducción

3.2.Objetivos del artículo científico $1 \quad 67$

3.3. Artículo científico $1 \quad 68$

3.4.Discusion de los resultados $\quad 77$

3.5.Conclusiones del artículo científico 1

CAPÍTULO 4: Síntesis del pigmento de coloración rojanaranja basado en $\mathrm{Y}_{2} \mathrm{Zr}_{2} \mathrm{O}_{7}$ dopado con $\operatorname{Pr}$ y $\mathrm{Fe}$

4.1.Introducción

4.2.Objetivos del artículo científico 2 90

4.3. Artículo científico 2

4.4.Discusion de los resultados 101

4.5.Conclusiones del artículo científico 2

CAPÌTULO 5: Conducción electrónica en conductores de ion

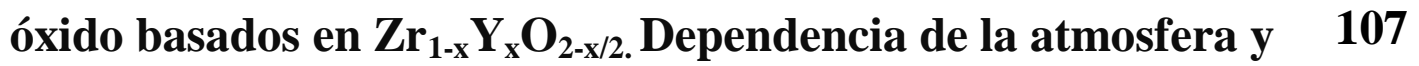
del voltaje aplicado

5.1.Introducción

5.2. Objetivos del artículo científico $3 \quad 111$

5.3. Artículo científico $3 \quad 112$

5.4. Discusión de los resultados 121

5.5.Conclusiones del artículo científico $3 \quad 126$ 
Anexo I: Técnicas de caracterización 137

A1.1.Difracción de rayos X, método del polvo cristalino 139

A1.2.Microscopía electrónica de barrido (SEM) y EDX 141

A1.3. Espectroscopia ultravioleta-visible-infrarrojo cercano 143

A1.4. Colorimetría 144

A1.5. Espectroscopia de fluorescencia 145

A1.6.Espectroscopia Fotoelectrónica de Rayos X (XPS) 148

A1.7. Espectroscopia de impedancia 152

Anexo II: Notación de Kröger-Vink 163

A2.1. Notación de Kröger-Vink 165

$\begin{array}{ll}\text { Bibliografía } & 167\end{array}$

$\begin{array}{ll}\text { Renuncia expresa de los coautores } & 183\end{array}$ 



\section{ÍNDICE DE FIGURAS}

Figura 1. Celda unidad parcial del pirocloro. Las esferas azules representan los

cationes $\mathrm{A}^{3+}$, las amarillas a $\mathrm{B}^{4+}$ y las rojas los aniones $\mathrm{O}^{2}$.

Figura 2. Celda unidad parcial de la estructura fluorita defectuosa. Las esferas azules

representan los cationes $\mathrm{A}^{3+}$, las amarillas a $\mathrm{B}^{4+}$ y las rojas los aniones $\mathrm{O}^{2-}$.

Figura 3. Diagrama de equilibrio de fase $\mathrm{ZrO}_{2}-\mathrm{YO}_{1.5}$.

Figura 4. Polimorfismo de la circona.

Figura 5. Representación visual de la introducción de óxido de itrio en la estructura de la circona, generando vacantes de oxígeno en las posiciones tetraédricas.

Figura 6. Diagrama de fases $\mathrm{ZrO}_{2}-\mathrm{YO}_{1.5}$ de la zona rica en $\mathrm{ZrO}_{2}$.

Figura 7. Diagrama de fases $\mathrm{ZrO}_{2}-\mathrm{Y}_{2} \mathrm{O}_{3}$ de la zona rica en $\mathrm{ZrO}_{2}$.

Figura 8. Distintos grados de gelificación de un gel polimérico inorgánico formado a partir de dos alcóxidos de metales diferentes.

Figura 9. Ejemplo de longitudes de onda reflejadas en una zanahoria.

Figura 10. Gráfico de Arrhenius para diferentes composiciones del sistema $\mathrm{Y}_{2} \mathrm{O}_{3}-\mathrm{ZrO}_{2}$.

Figura 11. Representación esquemática de los dominós electrónicos y iónicos en función de la presión parcial de oxígeno. 
Figura 12. a) Diagrama de bandas para un metal y un semiconductor tipo $\mathrm{n}$ antes del

contacto. b) Diagrama de bandas para un metal y un semiconductor tipo $\mathrm{n}$ en contacto, donde los niveles de Fermi de ambo se igualan formando la barrera de Schottky por inyección de electrones del semiconductor al metal. c) Esquema de una barrera de Schottky, observándose la "depleted región”, presentando un comportamiento muy similar a una unión pn. Donde $\mathrm{E}_{0}$ es el nivel de vacío, $\mathrm{E}_{\mathrm{FM}} \mathrm{y}$ $\mathrm{E}_{\mathrm{FS}}$ son los niveles de fermi del metal y el semiconductor respectivamente, $\mathrm{E}_{\mathrm{C}}$ es la banda de conducción y $E_{V}$ la banda de valencia. $\Phi_{\mathrm{M}} \Phi_{\mathrm{S}}$ son las funciones trabajo del metal y del semiconductor respectivamente.

Figura 13. Circuitos equivalentes teóricos usados como modelo (a y b); medidas de impedancia $Z^{*}$ experimentales (azul) y simulación teórica (rojo) (c y d); transporte electrónico en función del voltaje aplicado (f).

Figura 14. Diagrama de niveles energéticos y transiciones del $\mathrm{Tb}^{3+}$.

Figura 15. Diagrama de niveles energéticos y transiciones del $\operatorname{Pr}^{3+}$. 


\section{ARTICULOS RELACIONADOS CON ESTA TESIS}

"Pigments based on $\mathrm{Cr}$ and $\mathrm{Sb}$ doped $\mathrm{TiO}_{2}$ prepared by microemulsion-mediated solvothermal synthesis for inkjet printing on ceramics". Marc Jovaní, Maria Domingo, Thales R. Machado, Elson Longo, Héctor Beltrán-Mir, Eloisa Cordoncillo. Dye. Pigment, 116, 106-113 (2015)

"New red-shade environmental-friendly multifunctional pigment based on $\mathrm{Tb}$ and Fe doped $\mathrm{Y}_{2} \mathrm{Zr}_{2} \mathrm{O}_{7}$ for ceramic applications and cool roof coatings". Marc Jovaní, Ana Sanz, Héctor Beltrán-Mir, Eloisa Cordoncillo, Dye.Pigments 133, 33-40 (2016)

"Atmosphere- and Voltage-Dependent Electronic Condcutivity of Oxide-IonConducting $\operatorname{Zr}_{1-x} Y_{x} O_{2-x / 2 .}$ " Marc Jovaní, Héctor Beltrán-Mir, Eloisa Cordoncillo, Anthony R. West. Inorg. Chem., 56,7081-7088 (2017)

"Environmental-friendly red-orange ceramic pigment based on $\mathrm{Pr}$ and $\mathrm{Fe}$ codoped $\mathrm{Y}_{2} \mathrm{Zr}_{2} \mathrm{O}_{7}$ ". Marc Jovaní, Maria Fortuño Morte, Héctor Beltrán-Mir, Eloisa Cordoncillo, J. Eur, Ceram, Soc, 39, 2210-2217 (2018) 



\section{CAPÍTULO 1}

\section{Aspectos generales}

"Lo bueno de la ciencia es que es cierta, creas o no en ella" Neil de Grasse Tysson 



\subsection{Introducción}

En los últimos años los óxidos mixtos con estequiometría $\mathrm{A}_{2} \mathrm{~B}_{2} \mathrm{O}_{7}$, están siendo ampliamente estudiados debido a la gran variedad de propiedades que pueden presentar, tales como, alta estabilidad química, alta temperatura de fusión, baja temperatura de conducción, una excelente conducción iónica, etc. [1-6]. Estas propiedades hacen de este tipo de materiales altamente atractivos e interesantes para una gran variedad de aplicaciones [7]. Por ejemplo, una de las estructuras más ampliamente estudiadas en los últimos tiempos es el pirocloro $\mathrm{Y}_{2} \mathrm{Ti}_{2} \mathrm{O}_{7}$. Este pirocloro es un buen candidato para aplicaciones tales como: conductores iónicos de oxígeno [8], materiales alternativos para inmovilización de restos sólidos nucleares (en particular actínidos) [9], fotocatalizadores para la producción de hidrógeno a partir de agua [10], red huésped para incorporar cationes luminiscentes [11,12], red huésped para incorporación de cationes cromóforos en pigmentos cerámicos [13-15], etc. Por lo tanto, esta estequiometría posee una gran variedad de funcionalidades, hecho que permite su aplicación en diversos campos de la tecnología. No en vano, la importancia actual de este tipo de óxidos mixtos puede verse reflejada en el elevado número de publicaciones aparecidas en los últimos años en la literatura.

Los compuestos con estequiometría $\mathrm{A}_{2} \mathrm{~B}_{2} \mathrm{O}_{7}$ se pueden dividir en dos subgrupos dependiendo de la carga y del tamaño de los cationes. Se pueden encontrar los denominados pirocloros III-IV $\left(\mathrm{A}^{3+}\right)_{2}\left(\mathrm{~B}^{4+}\right)_{2} \mathrm{O}_{7}$ y los pirocloros II-V $\left(\mathrm{A}^{2+}\right)_{2}\left(\mathrm{~B}^{5+}\right)_{2} \mathrm{O}_{7}$. Los pirocloros III-IV con estados de oxidación $(+3,+4)$ son mucho más numerosos que los pirocloros II-V formados por iones $(+2,+5)$.

Además, los óxidos mixtos de tipo $\mathrm{A}_{2} \mathrm{~B}_{2} \mathrm{O}_{7}$ pueden presentar diferentes tipos de estructuras cristalinas, dependiendo de la relación de los radios iónicos (en la coordinación establecida para la estructura de pirocloro) de los elementos 
involucrados, es decir, $\mathrm{r}\left(\mathrm{A}^{3+}\right) / \mathrm{r}\left(\mathrm{B}^{4+}\right)$, dando lugar a estructuras tipo pirocloro, fluorita defectuosa o perovsquita laminar, presentando propiedades diversas en función de la estructura adoptada.

A presión atmosférica, se han establecido los límites que marcan la estabilidad de un tipo u otro de estructura. Así, la estructura pirocloro es estable cuando $\mathrm{r}\left(\mathrm{A}^{3+}\right) / \mathrm{r}\left(\mathrm{B}^{4+}\right)$ se encuentra dentro del intervalo 1.46-1.78 [16,17]. Valores de $r\left(\mathrm{~A}^{3+}\right) / \mathrm{r}\left(\mathrm{B}^{4+}\right)<1.46$ originan un desorden de las subredes catiónicas y aniónicas dando lugar a una estructura de fluorita defectuosa. Dicha transición ordendesorden, de pirocloro a fluorita, puede ser provocada además que por un cambio de composición, por un aumento de temperatura [18], presión [19] o también mediante radiaciones altamente energéticas [20].

Por otro lado, cuando la relación $\mathrm{r}\left(\mathrm{A}^{3+}\right) / \mathrm{r}\left(\mathrm{B}^{4+}\right)>1.78$, estos óxidos adquieren la estructura de perovsquita laminar. La fórmula general de esta familia de perovsquitas es $\mathrm{A}_{\mathrm{n}} \mathrm{B}_{\mathrm{n}} \mathrm{O}_{3 \mathrm{n}+2}$, donde $\mathrm{n}$ es el número de capas octaédricas. Su estructura se caracteriza por presentar octaedros $\left(\mathrm{BO}_{6}\right)$ compartiendo vértices, donde los cationes A pueden ocupar dos posiciones diferentes, o bien localizados en la estructura de la perovsquita, es decir, coordinados dodecaedricamente dentro de las capas de la perovsquita, o bien entre las regiones de las capas adyacentes, presentando un índice de coordinación seis y una geometría de prisma trigonal distorsionado [16,21].

Como consecuencia de lo anterior, la estequiometría de $\mathrm{A}_{2} \mathrm{~B}_{2} \mathrm{O}_{7}$ posee una versatilidad de composiciones y propiedades, haciendo uso del paradigma conocido: composición-estructura-propiedad.

La presente tesis aborda el estudio de óxidos mixtos de circonio e itrio, dentro del sistema $\mathrm{ZrO}_{2}-\mathrm{Y}_{2} \mathrm{O}_{3}$, mayoritariamente la estequiometría $\mathrm{Y}_{2} \mathrm{Zr}_{2} \mathrm{O}_{7}$. Esta estequiometría es capaz de albergar en su red gran variedad de cationes como dopantes, tanto en la posición del itrio como en la posición del circonio, lo cual le 
puede proporcionar una versatilidad de propiedades, y en consecuencia de aplicaciones, en diversos campos de la tecnología. Estas propiedades pueden ser moduladas en función de los cationes incorporados como dopantes, dependiendo de la propiedad que se pretenda desarrollar.

En el siguiente punto se abordará el estudio cristalográfico de las posibles estructuras adquiridas por las estequiometrías $\mathrm{A}_{2} \mathrm{~B}_{2} \mathrm{O}_{7}$ en función de la relación $\mathrm{r}\left(\mathrm{A}^{3+}\right) / \mathrm{r}\left(\mathrm{B}^{4+}\right)$, concretamente de las estructuras pirocloro y fluorita defectuosa, poniendo de manifiesto la importancia del valor adquirido por la relación $\mathrm{r}\left(\mathrm{A}^{3+}\right) / \mathrm{r}\left(\mathrm{B}^{4+}\right)$.

\subsection{La estequiometría $A_{2} B_{2} O_{7}$}

Como se ha comentado brevemente en la introducción, los óxidos mixtos de tipo $\mathrm{A}_{2} \mathrm{~B}_{2} \mathrm{O}_{7}$, presentan una gran variedad de propiedades diferentes las cuales les convierten en unos excelentes candidatos para su potencial aplicación en diferentes áreas de la tecnología. Estas propiedades son consecuencia de la estructura cristalina adoptada, lo cual a su vez, es una consecuencia directa del valor de la relación adquirida por los ràdios de los cationes $\mathrm{A}$ y $\mathrm{B}$.

Una estructura pirocloro ideal o completamente ordenada, presenta una estructura cúbica $F d-3 m$, con el mayor de los cationes ocupando la posición A y el catión más pequeño la posición $\mathrm{B}$, presentando en consecunecia dos tipos de oxígenos $\mathrm{A}_{2} \mathrm{~B}_{2} \mathrm{O}(1)_{6} \mathrm{O}(2)$. De acuerdo con la notación de Wyckoff, fijando el origen en el catión $\mathrm{B}^{4+}$, en la estructura pirocloro el catión $\mathrm{A}^{3+}$ ocupa la posición $16 d$ y el catión B la posición 16c, mientras que los dos diferentes tipos de oxígenos ocuparan la posición $48 f$ para $\mathrm{O}(1)$ y la posición $8 b$ para $\mathrm{O}(2)$, permaneciendo la posición $8 a$ desocupada [16,22]. Para una buena comprensión y visualización de 
dicha estructura, en la figura 1 se representa un octavo de la celda unidad del pirocloro.

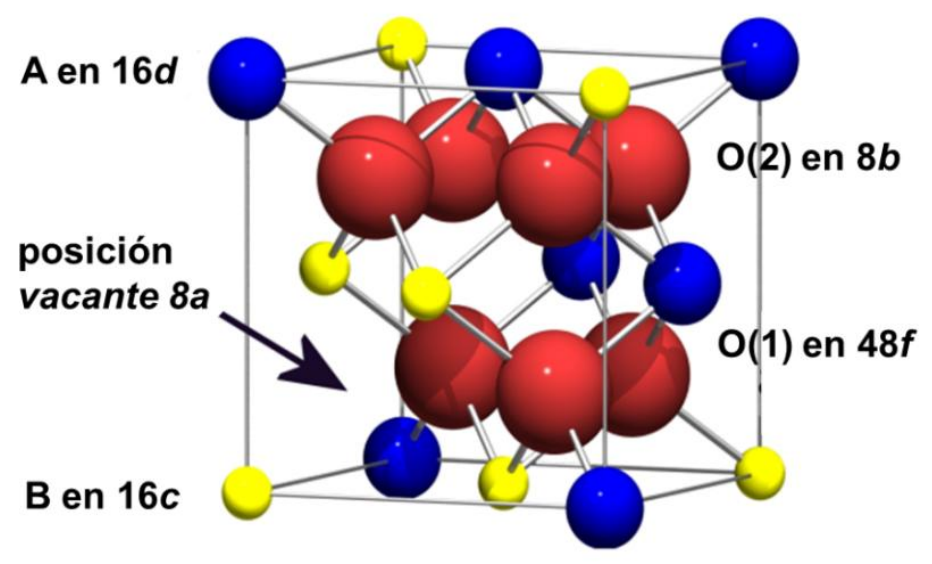

Figura 1. Celda unidad parcial del pirocloro. Las esferas azules representan los cationes $\mathrm{A}^{3+}$, las amarillas a $\mathrm{B}^{4+}$ y las rojas los aniones $\mathrm{O}^{2-}$.(Adaptación de la referencia [23].)

Una estructura estrechamente relacionada con la estructura pirocloro, es la fluorita defectuosa. La fluorita defectuosa, a diferencia de la estructura pirocloro, presenta las posiciones A y B cristalográficamente equivalentes, presentado un mayor grado de simetría pudiendo presentar vacantes de oxígeno no sólo en la posición $8 a$ sino en todas las posiciones de oxígeno.

La transición de la estructura pirocloro $(F d-3 m)$ a una estructura fluorita defectuosa $(\mathrm{Fm}-3 \mathrm{~m})$ es una transición de orden-desorden, que conlleva un desorden de los aniones $48 f, 8 b$ y la vacante $8 a$, así como un desorden de los cationes $16 c$ y $16 d$ [16]. Es decir, la estructura pirocloro se transforma en una estructura fluorita defectuosa mediante un desorden, provocando una distribución aleatoria tanto de los cationes como de los aniones. Este hecho genera un cambio en los índices de coordinación de los cationes, modificando tanto A como B con índices de 
coordinación de 8 y 6 respectivamente en la estructura pirocloro, a una media de 7 en la estructura fluorita defectuosa.

\section{La estequiometría $\mathrm{Y}_{2} \mathrm{Zr}_{2} \mathrm{O}_{7}$}

En el caso de la estequiometría que nos atañe en la presente tesis, el óxido mixto de itrio y circonio con una relación molar $\mathrm{Y}^{3+} / \mathrm{Zr}^{4+}$ de $50 / 50 \%$, cuya fórmula correspondería con la estequiometría $\mathrm{Y}_{2} \mathrm{Zr}_{2} \mathrm{O}_{7}$, la relación $\mathrm{r}\left(\mathrm{A}^{3+}\right) / \mathrm{r}\left(\mathrm{B}^{4+}\right)$ presenta un valor de 1.41, presentando por tanto una estructura cristalina tipo fluorita defectuosa. Esta fluorita defectuosa presenta un grupo espacial $F m-3 m$ con un parámetro de celda $\mathrm{a}=5.2042 \AA$ con $\mathrm{Z}=1$ [24]. De este modo, se podría decir que esta estructura de fluorita defectuosa consiste en una red cationes A y B distribuidos de una forma aleatoria en un ordenamiento cúbico. Sin embargo esta distribución aleatoria de los cationes A y B no es del todo cierta, ya que en el caso de la estequiometría $\mathrm{Y}_{2} \mathrm{Zr}_{2} \mathrm{O}_{7}$ se han predicho los índices de coordinación, aproximándose a 7.2 para el caso del catión $\mathrm{Y}^{3+}$ y 6.8 para el caso del catión $\mathrm{Zr}^{4+}$ [25]. La figura 2 muestra la representación de la celda unidad de una fluorita defectuosa.

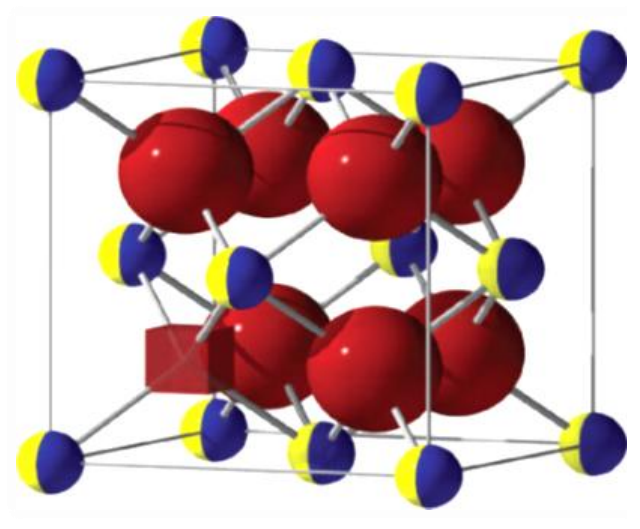

Figura 2. Celda unidad parcial de la estructura fluorita defectuosa. Las esferas azules representan los cationes $\mathrm{A}^{3+}$, las amarillas a $\mathrm{B}^{4+} \mathrm{y}$ las rojas los aniones $\mathrm{O}^{2-}[26]$. 
Además, se puede entender la estequiometría $\mathrm{Y}_{2} \mathrm{Zr}_{2} \mathrm{O}_{7}\left(\mathrm{Y}_{2} \mathrm{O}_{3} \cdot 2 \mathrm{ZrO}_{2}\right)$, como un óxido de circonio dopado con itrio, sustituyendo el itrio en la mitad de las posiciones del circonio en la estructura. Teniendo en cuenta dicha actuación y dada la importancia de conocer las fases y composiciones binarias presentes al combinar ambos óxidos, a continuación se abordará el estudio del diagrama de fases para el sistema $\mathrm{ZrO}_{2}-\mathrm{Y}_{2} \mathrm{O}_{3}$.

\subsection{Diagrama de equilibrio de fases para el sistema $\mathrm{ZrO}_{2}-\mathrm{Y}_{2} \mathrm{O}_{3}$}

Los compuestos pertenecientes al sistema $\mathrm{ZrO}_{2}-\mathrm{Y}_{2} \mathrm{O}_{3}$ han sido estudiados por diferentes autores.

En la tabla 1, se muestran los estudios más relevantes realizados hasta la actualidad.

Tabla 1. Resumen de los estudios más relevantes realizados en el sistema $\mathrm{ZrO}_{2}-\mathrm{Y}_{2} \mathrm{O}_{3}$.

\begin{tabular}{c|c|c} 
Año & Autores & Estudio \\
\hline 1951 & Duwes y col. [27] & Experimental \\
1963 & Fan y col. [28] & Experimental \\
1975 & Scoot [29] & Experimental \\
1983 & Pascual y col.[30] & Experimental \\
1988 & Stubican [31] & Experimental \\
1988 & Degtyarev y col. [32] & Teórico \\
1990 & Du y col. [33] & Teórico \\
2004 & Chen y col.[34] & Teórico
\end{tabular}


Antes de abordar el estudio de las diversas zonas del diagrama de equilibrio de fases, se debe destacar como bien es conocido, el principal problema de la reacción sólido-sólido, que es de tipo cinético. Estas reacciones están gobernadas por procesos de difusión iónica, siendo estos procesos extremadamente lentos, pudiendo ser acelerados por un aumento de la temperatura. Esta problemática, conlleva que los límites de las diferentes fases sólo se puedan establecer de una manera fiable a altas temperaturas. A temperaturas más bajas, debido a este problema se debe recurrir a cálculos teóricos [34], o métodos de síntesis con reactividades mayores que la reacción sólido-sólido, como por ejemplo la metodología de síntesis sol-gel (utilizada en la presente tesis).

Como se observa en la tabla 1, el primer diagrama de fases para el sistema fue publicado en 1951 por Duwez y col. [27]. A este le siguieron posteriormente una gran variedad de publicaciones entre las que podemos destacar las de Stubican [36] y de Degtyarev y Voronin [32]. Ya en la década de los 90 han sido muchas las publicaciones, las cuales han ido aportando nuevas evidencias en zonas del diagrama que no habían sido aún establecidas [33,34,35,37,38]. En la actualidad todavía existen algunas zonas del diagrama que son desconocidas.

En 2004, Chen y col. [34] diseñaron un diagrama de equilibrio de fases para el sistema $\mathrm{ZrO}_{2}-\mathrm{YO}_{1.5}$, el cual plasma las investigaciones experimentales realizadas hasta la fecha junto a cálculos teóricos termodinámicos, siendo por tanto el más reciente. La figura 3 muestra el diagrama de equilibrio resultante de dicho estudio $\mathrm{ZrO}_{2}-\mathrm{YO}_{1.5}$. Con el objetivo de describir el diagrama de una forma clara, se ha realizado la descripción del mismo de izquierda a derecha, es decir, desde la parte rica en $\mathrm{ZrO}_{2}$ hacia mayores cantidades de itrio. Por lo tanto, dada la importancia de conocer los cambios estructurales que se producen al añadir el itrio, parece necesaria la descripción de las diferentes fases adquiridas por la circona en ausencia de itrio en su estructura. 


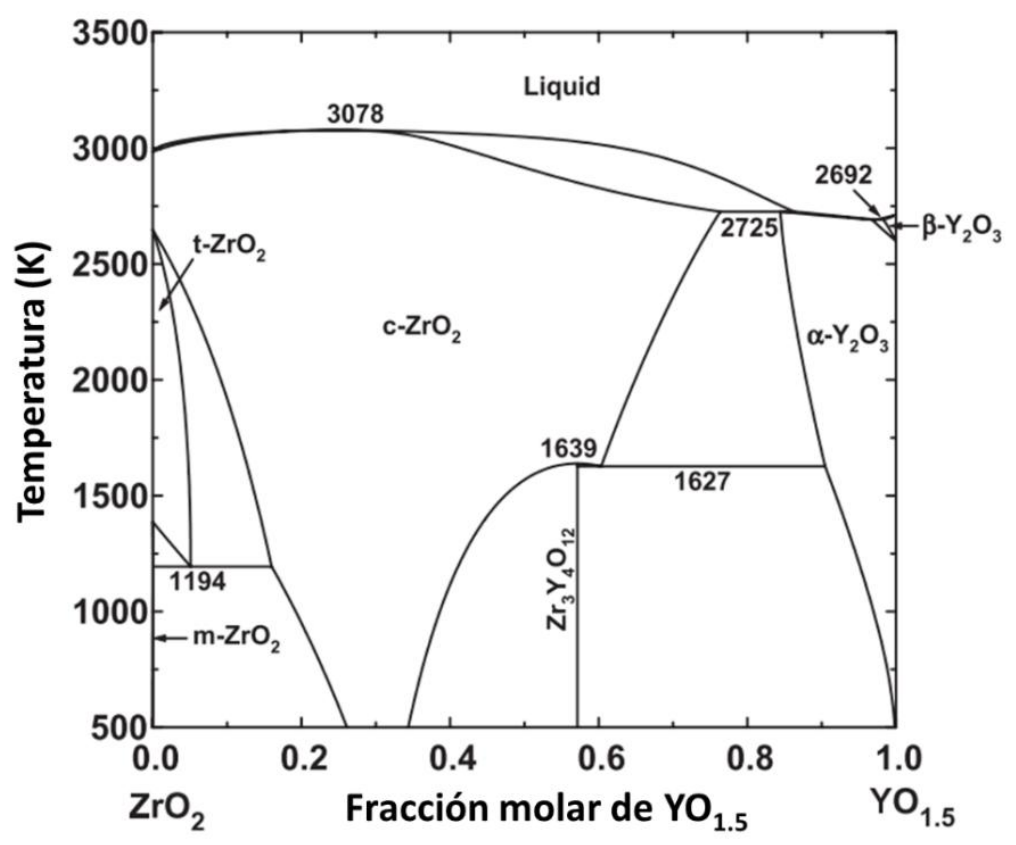

Figura 3. Diagrama de equilibrio de fase $\mathrm{ZrO}_{2}-\mathrm{YO}_{1.5}$ según Chen y col.(Adaptación de la referencia [34].)

\section{Polimorfismo de la circona}

La circona sin dopar presenta tres fases polimórficas, la fase monoclínica, la tetragonal y la cúbica [39].

La fase monoclínica es estable desde temperatura ambiente, hasta valores de $1170{ }^{\circ} \mathrm{C}$. A esta temperatura se produce la transición a la fase tetragonal, estable a temperaturas entre $1170{ }^{\circ} \mathrm{C}$ y $2370{ }^{\circ} \mathrm{C}$. La fase cúbica es estable desde $2370{ }^{\circ} \mathrm{C}$ hasta su temperatura de fusión alrededor de los $2680{ }^{\circ} \mathrm{C}$. La figura 4 muestra el polimorfismo de la circona, con las correspondientes celdas unidad de cada una de las estructuras cristalinas. 

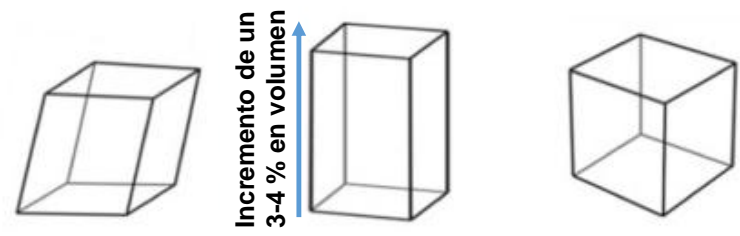

$\mathrm{ZrO}_{2}$

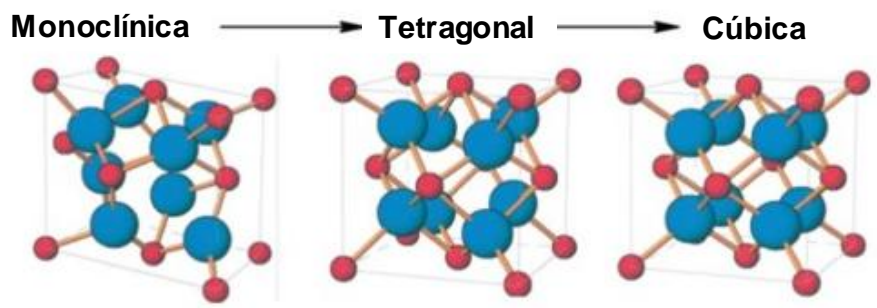

Figura 4. Polimorfismo de la circona. (Adaptación de la referencia [40].)

Como se observa en la figura 4 , las tres estructuras de la circona son parecidas a la estructura tipo fluorita, siendo la estructura de alta temperatura, la cúbica, la que presenta una estructura ideal. Sin embargo, tanto la fase tetragonal como la monoclínica muestran una ligera distorsión de la misma.

Con el objetivo de describir las fases cristalinas y las transformaciones que se producen en la circona, se han realizado diversos estudios. Se puede destacar el realizado por Kisi y Howard [41], estudiando los diferentes grupos espaciales, así como sus transformaciones. Adicionalmente diversos autores han determinado los parámetros de red de cada uno de los polimorfos de la circona [42,43]. En la tabla 2 se plasman a modo de ejemplo algunos de los resultados extraídos de los anteriores estudios. En la circona monoclínica $\left(\mathrm{m}-\mathrm{ZrO}_{2}\right)$, el $\mathrm{Zr}^{4+}$ presenta un número de coordinación de 7, teniendo dos tipos de coordinaciones con los distintos oxígenos, los denominados $\mathrm{O}(1)$ y $\mathrm{O}(2)$, presentando longitudes y ángulos de enlace distintos [42]. 
Tabla 2. Datos cristalográficos de la circona.

\begin{tabular}{c|c|c|c|c|c}
$\begin{array}{c}\text { Estructura } \\
\text { cristalina }\end{array}$ & Grupo & $\mathbf{a}(\mathbf{n m})$ & $\mathbf{b}$ & $\mathbf{c}$ & Otros \\
\hline Monoclínica & $\mathrm{P} 2{ }_{1} / \mathrm{C}(14)$ & 0.5156 & 0.5191 & 0.5303 & $\beta=98.9^{\circ}$ \\
Tetragonal & $\mathrm{P} 4{ }_{2} / \mathrm{nmc}(137)$ & 0.3624 & - & 0.5134 & $\mathrm{c} / \mathrm{a}=1.416$ \\
Cúbica & Fm-3m (225) & 0.5124 & - & - & -
\end{tabular}

En la fase tetragonal $\left(\mathrm{t}-\mathrm{ZrO}_{2}\right)$, el $\mathrm{Zr}^{4+}$ posee un número de coordinación 8 con cuatro iones óxido, situados a una distancia del ion circonio de $0.2065 \mathrm{~nm}$ y otros cuatro a $0.2455 \mathrm{~nm}$ [44].

La fase cúbica de alta temperatura $\left(\mathrm{c}-\mathrm{ZrO}_{2}\right)$, presenta una estructura cristalina de fluorita ideal, con el circonio rodeado de 8 oxígenos equidistantes, generando dos tetraedros iguales donde cada oxígeno se encuentra rodeado de cuatro circonios. En la figura 4 se muestran las celdas unidad de cada una de las estructuras cristalinas anteriormente mencionadas [40].

La transformación entre circona tetragonal $\left(\mathrm{t}-\mathrm{ZrO}_{2}\right)$ y monoclínica $(\mathrm{m}-$ $\mathrm{ZrO}_{2}$ ), tanto en el proceso de enfriamiento como de calentamiento, se produce mediante una trasformación martensítica [45]. Dicha transformación, lleva consigo un movimiento de átomos que da lugar a un cambio de forma en la región que se transforma, produciendo una distorsión de la propia estructura. La transformación producida al enfriar de circona tetragonal a monoclínica produce un aumento de volumen del 3-4\%. Sin embargo, en el cambio de monoclínica a tetragonal se produce el efecto contrario, es decir, la estructura se contrae. Estos cambios en el volumen producen un agrietamiento del material. Como consecuencia de ello, se evita el uso de ambos polimorfos en la fabricación de componentes cerámicos $[40,45]$. 
Estas tres fases de la circona presentan diferentes grados de noestequiometría, siendo la fase cúbica la que presenta un mayor desequilibrio estequiométrico. Dicha no-estequiometría generalmente debida a la deficiencia en oxígeno del material, hacen de esta fase la más estudiada por la gran cantidad de propiedades interesantes que presenta.

Por lo tanto, la formación de la fase cúbica, conlleva consigo una deficiencia de oxígeno en el material. La fase cubica presenta una elevada inestabilidad debido a la repulsión generada por los iones $\mathrm{O}^{2-}$ de gran tamaño, estabilizándose por la creación de vacantes, relajando dichas fuerzas de repulsión en la estructura [29,46,47]. En este sentido, el uso de dopantes, para la estabilización a temperatura ambiente de la fase cúbica de alta temperatura, parece una buena estrategia. Esta estabilización se produce por la sustitución del circonio (IV) por elementos de valencia menor, generando vacantes de oxígeno en la estructura con el objetivo de mantener la neutralidad eléctrica en el material $[48,49]$.

\section{Estabilización de la $\mathrm{ZrO}_{2}$ cúbica con iones itrio}

La formación de vacantes de oxígeno en la estructura juega un papel fundamental en la estabilización de la fase cúbica. En este sentido, la creación de vacantes en la estructura se genera mediante la adición de ciertos iones actuando como dopantes. Los óxidos más utilizados como fuente de iones dopantes son óxidos metálicos de alcalinotérreos como $\mathrm{MgO}$ y $\mathrm{CaO}$, o los óxidos de iones lantánidos e actínidos como $\mathrm{Sc}_{2} \mathrm{O}_{3}$ o $\mathrm{Y}_{2} \mathrm{O}_{3}$ [50]. De este modo la estructura cúbica tipo fluorita se estabiliza al añadir a la red cationes que sustituyen al circonio. Generalmente se utilizan cationes de menor valencia que el circonio, obligando a la estructura a compensar la carga eléctrica total del material, mediante la perdida de 
iones oxígeno. Dicha pérdida de oxígenos, genera huecos tetraédricos en la estructura fluorita, produciendo una relajación en las repulsiones de los iones $\mathrm{O}^{2-}$ y estabilizando la estructura cúbica [29,46,47]. Por tanto, el número de vacantes de oxígeno será proporcional a la cantidad de cationes dopantes que se añadan a la estructura.

Sin embargo, no sólo se puede conseguir la estabilización de la estructura cúbica mediante la generación de vacantes de oxígeno, sino también se puede estabilizar mediante la modificación de los parámetros de red. Por ejemplo mediante el uso de dopantes de igual valencia como $\mathrm{Ce}^{4+} \mathrm{o} \mathrm{Ge}^{4+}$, los cuales le confieren a la estructura una mayor rigidez [50]. Sin embargo, parece que la relajación estructural por pérdida de oxígeno también juega un papel fundamental en este caso [51].

El itrio es normalmente el catión más ampliamente utilizado para la estabilización de la fase cúbica de la circona. Así, agregando como dopante el óxido de itrio o itria $\left(\mathrm{Y}_{2} \mathrm{O}_{3}\right)$ a la estructura $\mathrm{ZrO}_{2}$ es posible estabilizar a temperatura ambiente la fase cúbica de la circona. La figura 5 muestra una representación visual de la introducción de $\mathrm{Y}_{2} \mathrm{O}_{3}$ en la estructura de $\mathrm{ZrO}_{2}$ con la correspondiente generación de vacantes de oxígeno [40].

El diagrama propuesto por Scott mostrado en la figura 6 [29], es el comúnmente aceptado para el sistema $\mathrm{ZrO}_{2}-\mathrm{Y}_{2} \mathrm{O}_{3}$ en la zona rica en $\mathrm{ZrO}_{2}$. Sin embargo, debido a la baja difusividad de los cationes en el método cerámico se debe recurrir a cálculos teóricos.

Como consecuencia de ello, no se conocen de una manera exacta los límites de la zona de existencia de las fases cúbica y tetragonal, sobre todo a bajas temperaturas, estimándose errores de al menos un 3-4\% en la proporción de las fases. 


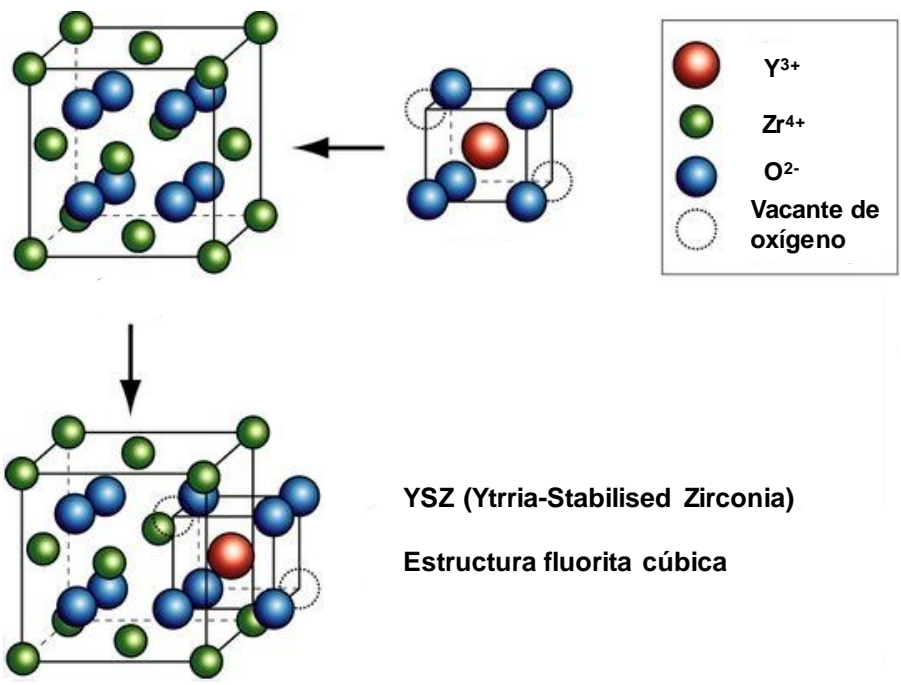

Figura 5. Representación visual de la introducción de óxido de itrio en la estructura de la circona, generando vacantes de oxígeno en las posiciones tetraédricas. (Adaptación de la referencia [40].)

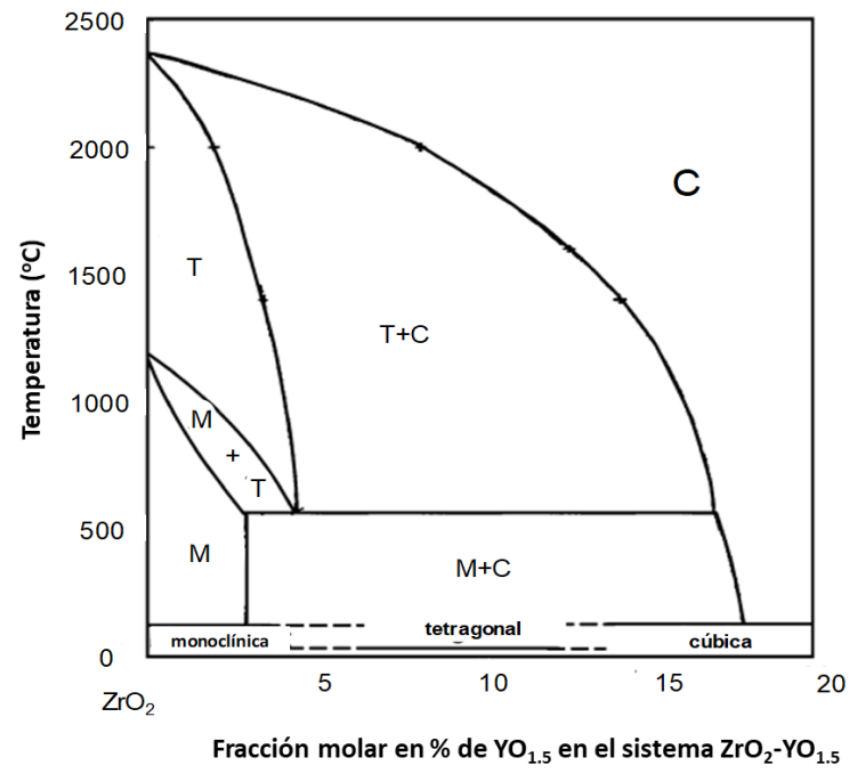

Figura 6. Diagrama de fases $\mathrm{ZrO}_{2}-\mathrm{YO}_{1.5}$ de la zona rica en $\mathrm{ZrO}_{2}$. (Adaptación de las referencias $[29,52]$.

Sin embargo, se ha demostrado experimentalmente por diversos autores, y teniendo en cuenta el error en los límites de las diferentes fases, que a cantidades superiores al $8 \%$ molar de $\mathrm{Y}_{2} \mathrm{O}_{3}$, se estabiliza la estructura cúbica [53]. Esta 
composición se referencia como YSZ08 en adelante. Debido a lo anteriormente expuesto una reconstrucción del diagrama de fases fue propuesto por Srivastava y col. [54]. Tal y como se muestra en la figura 7, este diagrama ha sido confirmado por diversos autores posteriormente $[53,55]$.

La figura 7 muestra el diagrama de fases para el sistema $\mathrm{ZrO}_{2}-\mathrm{Y}_{2} \mathrm{O}_{3}$ en la zona rica en $\mathrm{ZrO}_{2}$ y baja en $\mathrm{Y}_{2} \mathrm{O}_{3}$. En este diagrama la fracción molar de $\mathrm{Y}_{2} \mathrm{O}_{3}$ es la mitad que la utilizada en el diagrama anteriormente descrito por Scott [29], donde se representaba $\mathrm{ZrO}_{2}-\mathrm{YO}_{1.5}$.

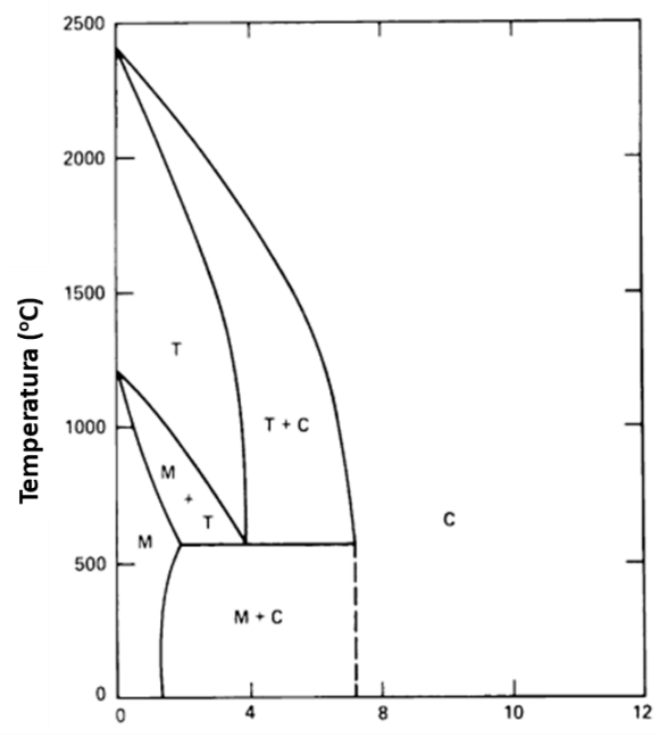

Fracción molar en \% de $\mathrm{Y}_{2} \mathrm{O}_{3}$ en el sistema $\mathrm{ZrO}_{2}-\mathrm{Y}_{2} \mathrm{O}_{3}$

Figura 7. Diagrama de fases $\mathrm{ZrO}_{2}-\mathrm{Y}_{2} \mathrm{O}_{3}$ de la zona rica en $\mathrm{ZrO}_{2}$. (Adaptación de las referencia [53].)

Como se observa en el diagrama de fases, a temperatura ambiente la circona dopada con itria adoptará fundamentalmente una estructura monoclínica en cantidades de $\mathrm{Y}_{2} \mathrm{O}_{3}$ bajas, hasta alrededor del $2 \%$. La proporción de fase monoclínica disminuye gradualmente con el incremento del contenido de $\mathrm{Y}_{2} \mathrm{O}_{3}$. Srikanth y Subbarao [56], determinaron mediante DRX (Difracción de rayos X) 
una cantidad muy baja de fase monoclínica cuando el dopaje con itria fue del 6\%, presentado esencialmente fase cúbica. Bajo ciertas condiciones se ha demostrado la posibilidad de obtener, fase metaestable tetragonal a temperatura ambiente. A mayores cantidades de dopaje del 9-12 \% se presenta una fase cúbica pura. La estabilización de la fase cúbica de la circona a temperatura ambiente se conoce comúnmente como circona estabilizada con itria. La fase cristalina presentada por este tipo de sistemas se ve fuertemente influenciada por la cantidad de itrio que se introduce en la red de la circona.

\section{Diagrama de equilibrio de fases $\mathrm{ZrO}_{2}-\mathrm{Y}_{2} \mathrm{O}_{3}$ a mayores concentraciones de itrio}

Como muestra el diagrama de fases $\mathrm{ZrO}_{2}-\mathrm{YO}_{1.5}$ (figura 3), la fase cúbica de la circona $\left(\mathrm{c}-\mathrm{ZrO}_{2}\right)$ disuelve un mayor contenido molar de itrio respecto a la fase monoclínica $\left(\mathrm{m}-\mathrm{ZrO}_{2}\right)$ y a la fase tetragonal $\left(\mathrm{t}-\mathrm{ZrO}_{2}\right)$, disolviendo un máximo de $70 \%$ molar de $\mathrm{YO}_{1.5}$ a la temperatura de $2452{ }^{\circ} \mathrm{C}(2725 \mathrm{~K})$. En cambio, la circona tetragonal sólo admite un 5-6\% molar de $\mathrm{YO}_{1.5}$ a una temperatura de $921{ }^{\circ} \mathrm{C}$ (1194 K). Por lo que se refiere a la solubilidad de $\mathrm{YO}_{1.5}$, en la circona monoclínica, el diagrama muestra una cantidad muy baja, casi inapreciable. No obstante Ruh y col. [57] han establecido un máximo de solubilidad alrededor del 3\%.

A mayores concentraciones de $\mathrm{YO}_{1.5}$, más allá de la fase $\mathrm{c}-\mathrm{ZrO}_{2}$, se observa la formación del compuesto estequiométrico $\mathrm{Zr}_{3} \mathrm{Y}_{4} \mathrm{O}_{12}$. Esta composición fue descrita por primera vez por Stubican y col. [36] y Scott [58], y fue posteriormente confirmada por Pascual y Duran [30] y Jayaratna y col. [59]. Esta estructura está estrechamente relacionada con la fluorita isoestructural con los compuestos $\mathrm{M}_{7} \mathrm{O}_{12}$, siendo el límite de temperatura de $1250{ }^{\circ} \mathrm{C}(1523 \mathrm{~K})$. Posteriormente Scoot [58] observó el desorden de esta fase a una fase fluorita alrededor de $1370{ }^{\circ} \mathrm{C}(1643 \mathrm{~K})$. Pascual y Duran observaron el mismo comportamiento pero una temperatura 
superior de $1375{ }^{\circ} \mathrm{C}(1648 \mathrm{~K})$, en la que fueron necesarias varias horas para su descomposición [30]. Más tarde Stubican [31] sugirió que la estequiometría $\mathrm{Zr}_{3} \mathrm{Y}_{4} \mathrm{O}_{12}$ adquiría una simetría romboédrica y que descomponía a $1250{ }^{\circ} \mathrm{C}$ (1523 K). Investigaciones posteriores, revisaron la descomposición de esta fase, concluyendo en la existencia de una descomposición a $1382{ }^{\circ} \mathrm{C}(1655 \mathrm{~K})$. Con el objetivo de aclarar esta situación Stubican y col. calcinaron mezclas de polvos reactivos con porcentajes molares de 71.0, 78.8, 85.7 y $91.9 \%$ de $\mathrm{YO}_{1.5}$ a $1573 \mathrm{~K}$ durante 6 meses. Sólo se detectaron mezclas de $\mathrm{Zr}_{3} \mathrm{Y}_{4} \mathrm{O}_{12}$ y de $\alpha-\mathrm{Y}_{2} \mathrm{O}_{3}$ cúbica [36].

A concentraciones superiores de itrio, en la zona rica en $\mathrm{YO}_{1.5}, \alpha-\mathrm{Y}_{2} \mathrm{O}_{3}$ disuelve alrededor de $14 \%$ de $\mathrm{ZrO}_{2}$ y la solubilidad del $\mathrm{ZrO}_{2}$ en $\beta-\mathrm{Y}_{2} \mathrm{O}_{3}$ no es conocida a ciencia cierta.

Por lo que se refiere a la estequiometría abordada en la presente tesis $\mathrm{Y}_{2} \mathrm{Zr}_{2} \mathrm{O}_{7}$, no aparece en el diagrama de fases. Sin embargo esta estequiometría, con una distribución cúbica de los cationes, ha sido detectada por Roth y col. [60] mediante difracción de rayos X (DRX) y posteriormente confirmada por Fan y col. [28]. Estudios realizados por difracción de neutrones por Smith y col. [61] contradicen las evidencias mostradas por Roth [60] y Fan y col.[28], no pudiendo demostrar la existencia de la estequiometría $\mathrm{Y}_{2} \mathrm{Zr}_{2} \mathrm{O}_{7}$ como una fase ordenada. Esta dificultad para la identificación de esta fase, puede venir dada por la baja difusión que presentan los cationes de circonio e itrio por el método cerámico, requiriendo temperaturas muy elevadas o tiempos muy largos de calcinación. En este sentido existen estudios en los cuales se ha demostrado la posibilidad de sintetizar la estequiometría $\mathrm{Y}_{2} \mathrm{Zr}_{2} \mathrm{O}_{7}$ mediante métodos de síntesis diferentes al método cerámico, los cuales poseen una mayor reactividad y una mejor difusión de los cationes, tales como métodos de combustión [62-64] o síntesis hidrotermal [65].

En este sentido la metodología de síntesis vía sol-gel parece una metodología óptima para la síntesis de la estequiometría $\mathrm{Y}_{2} \mathrm{Zr}_{2} \mathrm{O}_{7}$, ya que presenta una serie de ventajas respecto al método cerámico debido a sus características, 
permitiendo la obtención de materiales no sintetizables por el método cerámico tradicional [66].

Por lo tanto, en el siguiente punto se describen las características generales del método sol-gel, utilizado en la presente tesis.

\subsection{Método de síntesis}

Debido a la demanda creciente desde el punto de vista tecnológico, en los sectores cerámicos se han ido desarrollado nuevos métodos de síntesis alternativos al método cerámico tradicional. Se ha alcanzado un alto grado de tecnología en campos de aplicación que hubiesen sido impensables años atrás [67]. En este sentido, el desarrollo de otros métodos de síntesis ha jugado un papel fundamental y han sufrido un gran avance desde el método cerámico tradicional hasta las metodologías no convencionales conocidas en la actualidad. Los métodos de síntesis vía sol-gel, coprecipitación, solvotermal/hidrotermal, permiten conseguir materiales con mayor pureza y homogeneidad a menores temperaturas y con un mayor control de las características finales del material que en el método cerámico. Incluso permiten la obtención de nuevos materiales difíciles de preparar por el método cerámico tradicional [66]. En la presente tesis se ha utilizado la metodología de síntesis sol-gel polimérico, por tanto, se va a describir en mayor detalle dicho método de síntesis.

En la denominación del procesado sol-gel se incluyen todas aquellas técnicas que utilizan un sol o un gel como etapa intermedia en la obtención de un determinado material. Inicialmente se tiene un sol, frecuentemente definido como una suspensión de partículas pequeñas y discretas que permanecen dispersadas en una fase líquida, que sufre una transformación transformándose en un gel, 
caracterizado por una estructura tridimensional infinita que se extiende uniformemente a través del medio líquido. Para ello se pueden seguir dos vías: la ruta coloidal y la ruta polimérica. Puesto que en la presente tesis sólo se va usar la síntesis sol-gel polimérica, se va a describir sólo esta última. En este caso los geles formados son macromoléculas muy grandes en equilibrio con el sol y que se obtienen a partir de un proceso de polimerización.

En esta ruta los geles se desarrollan a partir de sucesivas reacciones de hidrólisis, condensación y polimerización. Estos geles poliméricos se sintetizan casi exclusivamente a partir de alcóxidos de los elementos de la composición que se quiere preparar, aunque también pueden utilizarse sales u óxidos en menor medida. Los alcóxidos se disuelven en un disolvente, generalmente alcohol, formándose el sol de una forma homogénea [66].

Las reacciones importantes que se producen durante el proceso son las de hidrólisis y condensación-polimerización, formando cadenas poliméricas que al evolucionar forman redes de óxidos polimerizados.

Las reacciones de hidrólisis se producen por el ataque de un grupo hidroxilo de la molécula de agua en el átomo metálico, mediante la reacción 1, donde $\mathrm{M}(\mathrm{OR}) \mathrm{n}$ es el alcóxido metálico, $\mathrm{M}$ es el catión metálico y $\mathrm{R}$ es el grupo alquilo.

$$
\mathrm{M}(\mathrm{OR})_{\mathrm{n}}+\mathrm{xH}_{2} \mathrm{O} \rightarrow \mathrm{M}(\mathrm{OH})_{\mathrm{x}}(\mathrm{OR})_{\mathrm{n}-\mathrm{x}}+\mathrm{xROH}
$$

Las reacciones de hidrolisis vienen seguidas de las reacciones de condensación para formar las especies M-O-M a través de la deshidratación (reacción 2) o la desalcoholación (reacción 3). La deshidratación conlleva condensación por formación de puentes hidroxo (-OH) entre los dos centros 
metálicos, mientras que la desalcoholación es la condensación a través de puentes de oxígeno.

$$
\begin{aligned}
& -\mathrm{M}-\mathrm{OH}+\mathrm{HO}-\mathrm{M}-\rightarrow-\mathrm{M}-\mathrm{O}-\mathrm{M}-+\mathrm{H}_{2} \mathrm{O} \\
& -\mathrm{M}-\mathrm{OH}+\mathrm{RO}-\mathrm{M} \rightarrow-\mathrm{M}-\mathrm{O}-\mathrm{M}-+\mathrm{ROH}
\end{aligned}
$$

El mecanismo de todas estas reacciones influirá en la transformación del alcóxido en una red macromolecular. Dependiendo del control de las reacciones anteriores se pueden obtener tanto cadenas lineales como estructuras entrecruzadas. La obtención de un tipo u otro de estructura depende fundamentalmente de las velocidades relativas de hidrólisis y condensación, cuanto más lenta sea la reacción de hidrólisis comparada con la de condensación más se tenderá a la obtención de cadenas lineales. De este modo la estructura y la morfología del material resultante dependerá en gran medida de la contribución de cada una de estas reacciones. Por ello es fundamental controlar diversos factores como los precursores utilizados, la razón molar agua/alcóxido, el uso de catalizadores, adición de agentes complejantes, $\mathrm{pH}$, temperatura, disolvente, etc.[66].

La elección de los alcóxidos de partida es primordial para la consecución de un buen gel. Cuando se quiere realizar un gel de este tipo con más de un ion metálico, hay que tener en cuenta que el grado de homogeneidad del gel resultante, estará fuertemente influenciado no sólo por el nivel de homogeneización de los diferentes alcóxidos, sino también por la reactividad de cada uno de los alcóxidos, formándose desde un gel homogéneo, hasta una mezcla heterogénea de geles, o especies con diferentes grados de hidrólisis, como muestra la figura 8. 


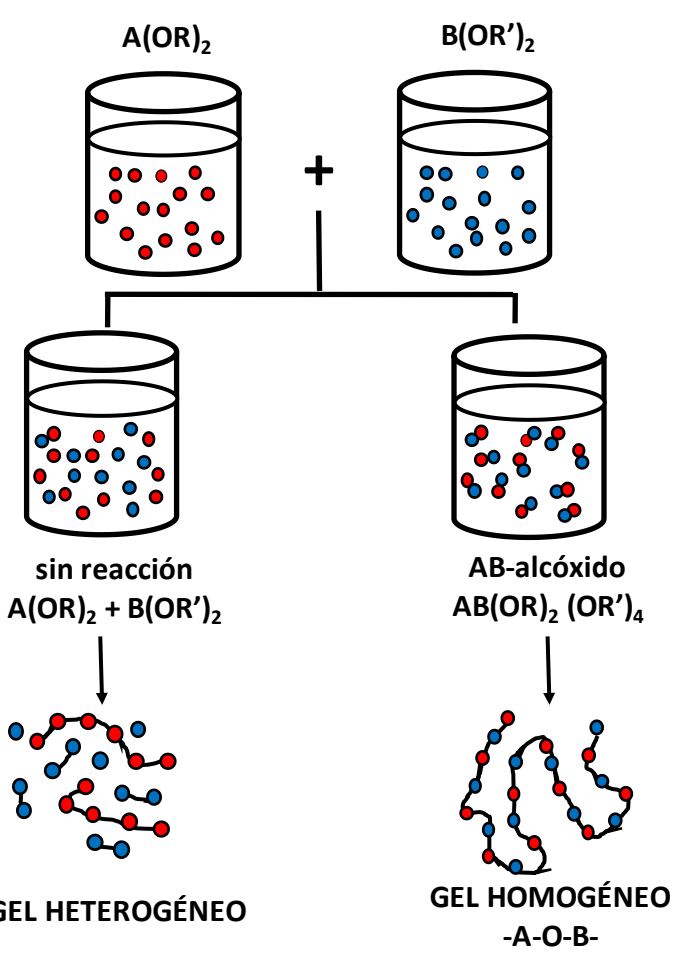

Figura 8. Distintos grados de gelificación de un gel polimérico inorgánico formado a partir de dos alcóxidos de metales diferentes.

Para adecuar la reactividad de los precursores se pueden emplear diferentes estrategias:

- Utilizar reactivos orgánicos como ácidos orgánicos o acetilacetona. La complejación del metal permite disminuir la funcionalidad del sistema y le protege frente la hidrólisis y la condensación, por aumento de su índice de coordinación $[68,69]$.

- Disminuir el pH de reacción favoreciendo la rotura de los puentes oxo o hidroxo (peptización del óxido). 
- Reducir la cantidad de agua "in situ”, o bien utilizando micelas inversas en el medio de reacción [70].

La elección del disolvente también es muy importante, influyendo fuertemente el proceso de gelificación. Los disolventes más comunes son alcoholes, eligiéndose por lo general los que tienen una presión de vapor alta. Hay que tener en cuenta, que el proceso final consiste en la eliminación del disolvente (secado) y posterior tratamiento térmico. El secado del gel está influenciado por el material que se pretende obtener, pero es bastante independiente del grado de cristalinidad o del estado de descomposición del gel [71].

Cuando el gel ya está seco, este debe ser tratado térmicamente para obtener el material cerámico final. Como todas las técnicas de preparación la vía sol-gel presenta ventajas e inconvenientes, en general podemos considerar como ventajas del método:

- La alta homogeneidad del producto final.

- La elevada pureza de los precursores, que permite un control de la nucleación y el crecimiento y por lo tanto un control del tamaño, distribución y la forma de las partículas.

- Bajas temperaturas de reacción, en comparación con otros métodos como el cerámico.

Por lo que se refiere a los inconvenientes, la mayor desventaja del método consiste en el alto coste de los precursores a utilizar en comparación a otros métodos como el método cerámico. Sin embargo, este coste económico adicional se contrarresta por las características optimas obtenidas del producto final, de una pureza mucho mayor y unas propiedades modulables, según los parámetros de 
síntesis, lo que comporta un valor añadido frente a otros métodos de síntesis tradicionales, como puede ser el método cerámico tradicional.

Por lo tanto, el método sol-gel polimérico confiere la posibilidad de sintetizar diferentes composiciones del sistema $\mathrm{ZrO}_{2}-\mathrm{YO}_{1.5}$ que difícilmente se pueden preparar por métodos menos reactivos, como el método cerámico tradicional. Esta posibilidad proporciona una variedad de estequiometrías las cuales potencialmente son capaces de abordar algunas problemáticas surgidas en diversas áreas en ciencia de materiales. En la presente tesis se aprovecha la multifuncionalidad de estos óxidos mixtos, para el estudio de su potencial uso en el campo de los pigmentos cerámicos, así como de las electrocerámicas. En consecuencia, en el siguiente punto, se abordará la problemática presente en el campo de los pigmentos cerámicos, así como el potencial de los compuestos con estequiometría $\mathrm{A}_{2} \mathrm{~B}_{2} \mathrm{O}_{7}$ para resolver algunos de los problemas que se plantean en el mismo.

\subsection{Pigmentos cerámicos}

Los pigmentos y colorantes han sido utilizados durante toda la historia de la humanidad y desde la antigüedad han estado directamente asociados a las manifestaciones culturales de las distintas civilizaciones. Desde los inicios de la alquimia ha existido relación entre esta y la producción de nuevos pigmentos. Dicha vinculación ha favorecido la obtención de numerosos pigmentos de origen artificial, y la paulatina aparición de estos nuevos productos ha permitido aumentar de una forma amplía la gama de colores utilizados [72]. Es bien conocido, que ya desde la época paleolítica eran utilizados los pigmentos de origen natural, de naturaleza inorgánica, como el ocre rojo [hematites $\left.\left(\alpha-\mathrm{Fe}_{2} \mathrm{O}_{3}\right)\right]$, ocre amarillo [goetita $\left(\alpha-\mathrm{FeO}(\mathrm{OH})\right.$ ], negro carbón y calcita $\left(\mathrm{CaCO}_{3}\right)$. 
En la actualidad se define como pigmento cerámico aquellos sólidos cristalinos e inorgánicas capaces de desarrollar color y de estabilizarlo a altas temperaturas. Estos deben presentar algunas características tales como, ser térmicamente estables, resistentes a los ataques agresivos causados por ejemplo por los vidriados, además de presentar una granulometría adecuada y no desarrollar emanaciones de gases en el seno de los vidriados [73]. Desde un punto de vista estructural, un pigmento cerámico está formado por una red huésped y uno o varios iones cromóforos, responsables del color. Además, también pueden incorporar posibles modificadores con el objetivo de estabilizar o resaltar el color u otras propiedades físicas, sin producir cambios en la estructura.

Los cromóforos comúnmente más utilizados se caracterizan por presentar electrones en orbitales $d$ (metales de transición) posibilitando fenómenos tales como, las transiciones electrónicas $d-d$ y por presentar transferencias de carga entre el ligando y el metal. Ambos fenómenos son responsables de la aparición del color en un sólido inorgánico. Por lo tanto, el color de un pigmento cerámico puede variar en función del número de coordinación del cromóforo, la naturaleza de su entorno y especialmente de su estado de oxidación [66].

Actualmente, en la industria de los pigmentos cerámicos, surgen una serie de problemáticas, las cuales vienen dadas en gran medida por las exigencias medioambientales actuales, así como por la necesidad de desarrollar nuevas coloraciones que cumplan dichas exigencias. En este sentido, sería interesante matizar la definición de pimento cerámico recién definida, adecuándola a las exigencias requeridas en el siglo XXI. A la definición anteriormente descrita, se le debería añadir como requisito el uso de elementos cromóforos no tóxicos, evitando todos aquellos elementos nocivos para la salud y para el medioambiente.

Adicionalmente, también se podría añadir la posibilidad, que la estructura pigmentante fuera capaz de aportar otra funcionalidad a parte del desarrollo del propio color, proporcionado un valor añadido al pigmento. No en vano en la 
actualidad son muchas las investigaciones encaminadas con el propósito de desarrollar materiales que además del desarrollo del color, aporten una serie de propiedades adicionales, proporcionado al material una nueva o nuevas funcionalidades. A este tipo de materiales se les suele denominar comúnmente como materiales multifuncionales y/o materiales inteligentes.

En el siguiente punto se van a exponer los diferentes mecanismos causantes del color en sólidos inorgánicos.

\subsubsection{Origen del color en sólidos inorgánicos}

Se puede definir el color, como la sensación visual que experimenta el ojo humano de los objetos cuando hay luz. El color, sobrepasa el ámbito de lo puramente físico y alcanza el de la psicología, ya que lo percibimos a través del sentido de la vista, así pues se puede catalogar como una propiedad psicofísica. El ojo responde a la interacción de la luz con un objeto y el cerebro se encarga de interpretar esta información [74].

Desde el punto de vista físico, el color se origina cuando un material absorbe ciertas longitudes de onda que inciden sobre ellos y se reflejan el resto, siendo el color la combinación de aquellas longitudes de onda que inciden en el ojo [66].

La figura 9 muestra las longitudes de onda reflejadas por una zanahoria, proporcionándole el color naranja característico en la raíz y verde en el tallo. 

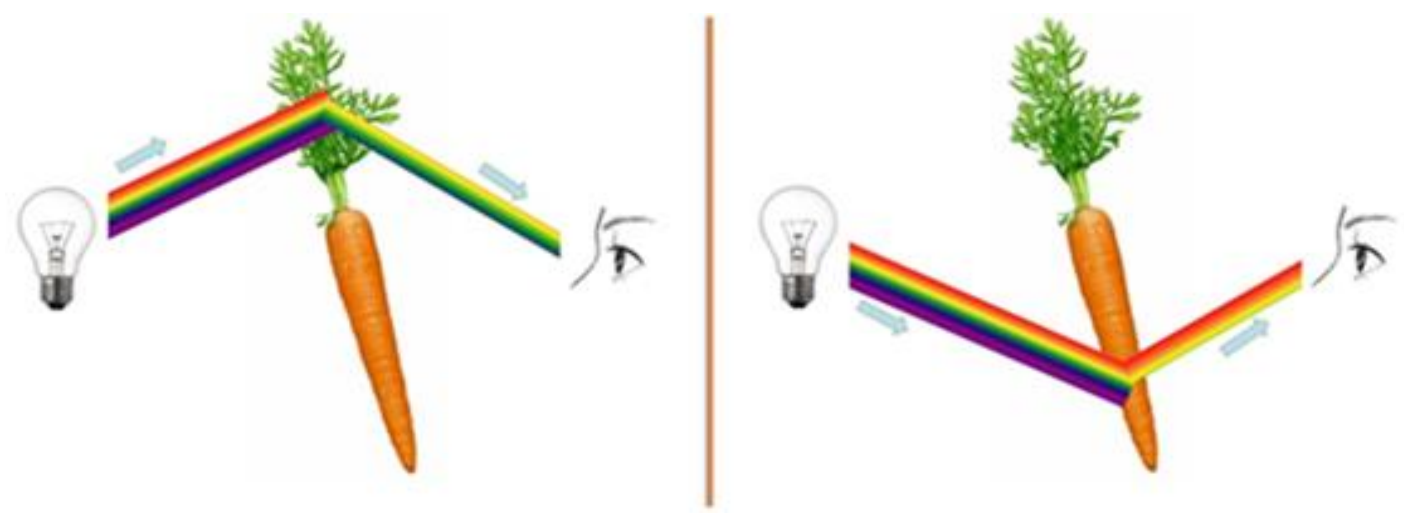

Figura 9. Ejemplo de longitudes de onda reflejadas en una zanahoria [75].

Los sólidos inorgánicos que poseen iones de metales de transición en su estructura, pueden absorber selectivamente longitudes de onda en la zona visible. Este hecho es debido a que presentan electrones en orbitales $d$ incompletos y por lo tanto posibilitan los dos fenómenos que son responsables del color, las transiciones electrónicas dentro de los niveles $d$ - $d$ y las transferencias de carga metal ligando. Las transiciones entre orbitales $d$ - $d$ del metal (transiciones $d$ - $d$ ) son de baja energía y aparecen habitualmente en la zona visible del espectro. En cambio en las transiciones de carga, un electrón de un orbital centrado en el ligando pasa a un orbital centrado en el metal o viceversa. En general estas transiciones aparecen en la región ultravioleta (UV), pero en ocasiones también pueden aparecer en la zona visible. Estos fenómenos explican la coloración presentada por compuestos que contienen elementos de transición en su estructura. No en vano, elementos de transición como el cromo, cobalto, vanadio, cadmio etc., han sido ampliamente utilizados en la industria de los pigmentos cerámicos. Sin embargo, estos elementos presentan problemas de toxicidad. Por lo tanto, en el siguiente punto se abordará una de las mayores problemáticas de la industria de los pigmentos cerámicos en la 
actualidad, que es la alta toxicidad de algunos de los elementos cromóforos utilizados, así como las posibles alternativas para su sustitución.

\subsubsection{Problemática de los pigmentos cerámicos en la actualidad}

Uno de los mayores retos de la industria de los pigmentos cerámicos en la actualidad, está centrado en la optimización de las propiedades ópticas de los pigmentos con tonalidad roja, así como el desarrollo de nuevos sistemas pigmentantes que presenten dicha tonalidad. Dicho reto, es debido a que la gran parte de los pigmentos rojos utilizados en la industria, no presentan una coloración roja "pura", sino que desarrollan coloraciones rosas o anaranjadas. Asimismo, estos pigmentos presentan como cromóforos metales de transición, tóxicos y/o contaminantes. Claros ejemplos de ello son: el corindón rosa de cromo-alúmina

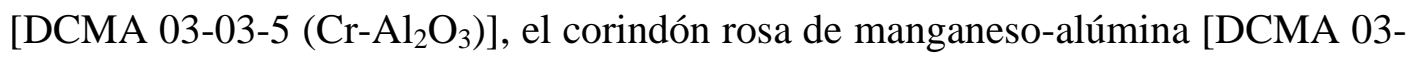
04-5 $\left(\mathrm{Mn}_{-} \mathrm{Al}_{2} \mathrm{O}_{3}\right)$ ], la casiterita malva de cromo-estaño [DCMA 11-23-5 (Cr$\left.\mathrm{SnO}_{2}\right)$ ], la esfena rosa de cromo-estaño [DCMA-12-25-5 ( $\left.\mathrm{Cr}-\mathrm{CaSnSiO}_{5}\right)$ ] y la espinela rosa de cromo-alúmina [DCMA-13-32-5 ( $\left.\mathrm{Zn}(\mathrm{Al}, \mathrm{Cr})_{2} \mathrm{O}_{4}\right)$ ] [76]. Todos ellos presentan el problema de no desarrollar una coloración rojiza pura, además de usar elementos de transición tóxicos tales como el cromo y el manganeso en sus estructuras.

El único pigmento cerámico conocido que presenta tonalidad roja "pura" es el sulfoseleniuro de cadmio $\left(\mathrm{CdSe}_{\mathrm{x}} \mathrm{S}_{1-\mathrm{x}}\right)$, el cual se encuentra encapsulado en una matriz de circón $\left(\mathrm{ZrSiO}_{4}\right)$. Este pigmento es altamente tóxico en su manufacturación, teniendo efectos negativos en el sistema reproductivo, respiratorio, locomotor además de ser una de las causas de diversos tipos de cáncer [77]. En consecuencia, en los últimos tiempos son muchos los esfuerzos realizados por la Unión Europea a través de la Agencia Europea de Sustancias y Mezclas 
Químicas (ECHA), con el objetivo de minimizar y/o reducir el uso de elementos tóxicos en la industria cerámica, siendo el cadmio una de las sustancias prohibidas por la regulación REACH (Registro, Evaluación, Autorización y Restricción de sustancias y mezclas químicas) [78].

Sin embargo, la inexistencia de otro pigmento que proporcione una coloración roja "pura", conlleva que sean numerosas las investigaciones para optimizar la síntesis de este pigmento tóxico, adaptándola a los requerimientos tecnológicos de la actualidad, más concretamente a las impresoras de chorro de tinta o industria "inkjet" [79,80].

Una de las alternativas utilizadas para la sustitución del sulfoseleniuro de cadmio, así como de los elementos altamente tóxicos utilizados como cromóforos en otros pigmentos, podría ser el uso de compuestos que contengan iones hierro como cromóforo. No en vano, es uno de los cromóforos más utilizados a lo largo de la historia, siendo todavía ampliamente utilizado en la industria actual. Este hecho es debido, a su gran disponibilidad en la naturaleza en forma de óxidos, no es tóxico y su precio es relativamente bajo. Estas características han hecho que estructuras que contienen cationes hierro, sean ampliamente utilizadas en una gran cantidad de productos industriales como fuente de color, como en cerámicas, pinturas, recubrimientos y plásticos [81].

En la industria actual es ampliamente usado el denominado "pink coral" o circón rosa de hierro-circonio [DCMA-14-44-5 (Fe- $\left.\mathrm{ZrSiO}_{4}\right)$, que como su propio nombre indica contiene hierro en su estructura [82]. El pink coral, presenta una coloración rojiza y no contiene en su estructura elementos tóxicos, ya que usa como elemento cromóforo el hierro, siendo estable a temperaturas superiores a $1200{ }^{\circ} \mathrm{C}$ y presentando una buena estabilidad química al ser mezclado en esmalte [83]. Sin embargo, este pigmento presenta otros tipos de problemas en términos de reproducibilidad. La coloración del pink coral es muy difícil de reproducir, en 
comparación con otros pigmentos cerámicos. Este grave problema de reproducibilidad, parece que tiene su origen en las etapas de la molturación de la circona y de la sílice, dependiendo el éxito en el color fuertemente del tamaño de partícula de los mismos, ya que a nivel industrial el método de síntesis utilizado en su producción es la reacción sólido-sólido [83,84]. Adicionalmente, otro de los problemas que presenta, es el desconocimiento del mecanismo de coloración, ya que no se conoce a ciencia cierta la posición del hierro en la estructura, ni su estado de oxidación. Existen diversos estudios, sobre ello pero con conclusiones dispares $[82,84,85]$. Por regla general es aceptado que el hierro se encuentra en estado de oxidación +3 , pero existe cierta controversia sobre el papel que juega el hierro en la formación de la red de circón y la posición que ocupa en la misma (substitucional o intersticial) $[82,86]$. Además, no desarrolla una coloración rojiza pura como la desarrollada por el sulfoseleniuro de cadmio.

En consecuencia, uno de los mayores retos de la industria de los pigmentos cerámico actual, radicaría en el desarrollo de nuevas estructuras pigmentantes de coloración rojiza, respetables con el medio ambiente y que no presenten problemas de reproducibilidad.

Sin embargo, ya en los últimos años han surgido otras áreas de aplicación, diferentes a la aplicación del pigmento en esmalte cerámico clásico. En este sentido existen gran variedad de aplicaciones de estos en diferentes materiales, como en pinturas y polímeros, donde se aplican por las propiedades que la mezcla con el pigmento cerámico le confiere al producto final. En el siguiente apartado se va a poner de manifiesto las diferentes áreas de aplicación donde los pigmentos cerámicos son potencialmente utilizables, y las propiedades que estos son capaces de conferir a los materiales a los que se aplican. 


\subsubsection{Nuevas aplicaciones de los pigmentos cerámicos}

Actualmente en el campo de la ciencia de materiales, son muchas las investigaciones encaminadas en el desarrollo de materiales que posean diversas propiedades en un mismo material, las cuales le permitan proporcionar diversas funcionalidades. Es decir, se están desarrollando una gran variedad de materiales multifuncionales. Se podría definir como material multifuncional aquellos que integran dos o más propiedades complementarias entre sí, con el fin de proveer ventajas que permitan desarrollar un sinfín de aplicaciones.

En este sentido el área de los pigmentos cerámicos, no es una excepción. Se puede destacar el desarrollo de los denominados pigmentos refrigerantes (cool pigments), los cuales son pigmentos de alta reflectividad en el infrarrojo cercano (NIR, 780-2100 nm). La aplicación de estos sobre la superficie de un edificio, conlleva un ahorro significativo en la climatización, protegiendo el edificio con una envolvente de revestimiento de alta reflectividad en la zona infrarroja [87-90]. Este aislamiento térmico es debido a que el material no presenta absorción en la región infrarroja. La radiación que alcanza a la tierra proveniente del Sol, presenta un intervalo de longitudes de onda de 295 a $2500 \mathrm{~nm}$. Este amplio rango de longitudes de onda se puede dividir en tres partes:

- Región Ultravioleta (295-400 nm): Se corresponde con el 5 \% de la energía solar que alcanza la superficie terrestre. Es la responsable de la degradación de las pinturas en los edificios.

- Región Visible (400-700 nm): Se corresponde al 50 \% de la energía solar. Absorciones en estas longitudes de onda son las responsables del color. 
- Región Infrarroja (700-2500 nm): El 45\% de la energía solar corresponde a esta región. No influye en el desarrollo del color. La consecuencia directa de la absorción de radiación infrarroja en los materiales es el calentamiento de los mismos. El calentamiento se produce sobretodo en el intervalo de 700 a $1100 \mathrm{~nm}[91]$.

Los denominados "cool pigments", son pigmentos inorgánicos capaces de reflejar la radiación en la región infrarroja, sin variar la absorción en la región visible, no provocando de este modo ninguna variación en el color presentado por el pigmento. Dicha propiedad les confiere la capacidad de evitar en gran medida un aumento de la temperatura del material sobre el que se depositará dicho pigmento. El uso de este tipo de pigmentos se ha incrementado notablemente para su aplicación en pinturas para tejados y fachadas de edificios. Esta medida contribuye a un ahorro del consumo energético, como por ejemplo en aparatos que generen aire acondicionado, destinados a enfriar las estancias, ya que reduce su uso considerablemente debido a un menor calentamiento de las mismas.

Además de una elevada reflectancia en la región infrarroja cercana (NIR), este tipo de pigmentos deben de poseer otro tipo de características, como una alta estabilidad e inercia química, ya que deben de resistir los ataques químicos a los que son sometidos, debido a su exposición al ambiente. Por lo tanto, no deben de sufrir perdida de color en presencia de gases contaminantes, tales como, $\mathrm{O}_{3}, \mathrm{SO}_{\mathrm{x}}$, $\mathrm{NO}_{\mathrm{x}} \mathrm{u}$ otros contaminantes comunes en los ambientes metropolitanos actuales. Esta característica de estabilidad química es imprescindible para la durabilidad del pigmento, ya que están diseñados para ser efectivos durante tiempos superiores a los 30 años. Estos pigmentos pueden ser utilizados en una gran variedad de aplicaciones como pinturas, tintas, plásticos, esmaltes cerámicos y vidrios [92]. 
En la actualidad el pigmento que presenta una mayor reflectancia en la zona del NIR está basado en la estructura de $\mathrm{TiO}_{2}$. Este pigmento de coloración blanca, presenta una reflectancia alrededor del $87 \%$ en la zona del infrarrojo cercano [93]. Sin embargo, debido a requerimientos estéticos se prefieren pigmentos que presenten una coloración diferente [94]. Dicha demanda, hace que se presente la misma problematica que en cualquier tipo de pigmento, el de la toxicidad de los cromóforos utilizados. La gran mayoría de los pigmentos utilizados como "cool pigments" en la actualidad, presentan iones metálicos tóxicos de elementos tales como $\mathrm{Co}, \mathrm{Pb}, \mathrm{Cd}, \mathrm{Cr}$, los cuales, como se ha visto, están restringidos por las regulaciones medioambientales [91,92,95].

Por consiguiente, análogamente al desarrollo de nuevos pigmentos cerámicos, el desarrollo de nuevos pigmentos inorgánicos con elevada reflectancia en la región infrarroja del espectro sin el uso de elementos dañinos para el medio ambiente, es otro de los retos en el campo de los pigmentos cerámicos.

Por todo lo anteriormente mencionado, son varios los retos que se presentan en la industria de los pigmentos cerámicos actual y parece que las composición $\mathrm{A}_{2} \mathrm{~B}_{2} \mathrm{O}_{7}$, pueden ser de gran utilidad con el objetivo de alcanzarlos, como se argumentará a continuación.

\subsubsection{La estructura tipo $A_{2} B_{2} O_{7}$ como red huésped de diferentes cromóforos}

Como se ha mencionado anteriormente, una de las redes huéspedes más prometedoras debido a la gran variedad de elementos que es capaz de incorporar en su red, es el óxido mixto $\mathrm{A}_{2} \mathrm{~B}_{2} \mathrm{O}_{7}$. Este tipo de óxidos mixtos presentan una estructura pirocloro / fluorita defectuosa / perovsquita laminar, dependiendo de la relación de radios $\left(\mathrm{A}^{3+}\right) / \mathrm{r}\left(\mathrm{B}^{4+}\right)$ que presenten. La posición $\mathrm{A}$ es ocupada por un 
catión grande (generalmente un lantánido) de baja valencia y B suele ser un metal de transición. El hierro parece ser un elemento idóneo para la incorporación en la estructura, ocupando parcialmente la posición B y actuando como elemento cromóforo, ya que presenta unas características y propiedades idóneas, debido a su configuración electrónica.

Otros tipos de elementos que serían apropiados considerar para una posible incorporación en la estructura, debido a sus características, son los elementos lantánidos. El uso de iones lantánidos en la industria de los pigmentos cerámicos se ha incrementado notablemente en los últimos años, debido a su nula toxicidad y sus buenas propiedades ópticas. Su uso, no sólo parece despertar interés en el campo de los pigmentos cerámicos, sino en una gran variedad de campos con aplicaciones tan diversas, como en el desarrollo de nuevos láseres [96,97], catalizadores [98,99], superconductores [100], conductores electrónicos [101], aplicaciones en medicina [102], etc.

Respecto al campo de los pigmentos cerámicos, los lantánidos son ampliamente utilizados en una gran variedad de estudios, sustituyendo a elementos de transición actuando como cromóforos o modificadores de la estructura en pigmentos cerámicos [103-105]. No en vano la principal aplicación industrial de los lantánidos es en el campo de los materiales cerámicos, consumiendo el $31 \%$ de la producción mundial. Claros ejemplos son el uso del $\mathrm{CeO}_{2}$ en los vidriados cerámicos, promoviendo la opacidad de los mismos o el uso del praseodimio en el pigmento amarillo de praseodimio circón $\left(\mathrm{Pr}-\mathrm{ZrSiO}_{4}\right)$, siendo el pigmento amarillo con mejor coloración producido industrialmente en la actualidad [106]. Su gran interés viene dado junto a su nula toxicidad, por la idoneidad de sus radios para su incorporación en una gran variedad de estructuras, junto a las propiedades ópticas que son capaces de aportar. Los elementos lantánidos, presentan la suma de las tres primeras energías de ionización relativamente bajas, por lo que son fuertemente 
electropositivos, presentando con facilidad un estado de oxidación de +3 , aunque algunos también puedan adoptar el estado de oxidación +4 . Los iones lantánidos trivalentes ([Xe] $4 f^{n}$ ), implican la actividad de los electrones $f$ fuertemente protegidos por las capas $5 \mathrm{~s}$ y 5 p. En consecuencia, el campo cristalino resultante por la interacción con los iones vecinos es muy bajo, por lo que a menudo el espectro de absorción de los compuestos lantánidos presenta bandas muy débiles. Algunas de las investigaciones más recientes en el campo de los pigmentos basadas en el uso de lantánidos, son el desarrollo de un pigmento de coloración marrón usando $\mathrm{La}^{3+}$ como dopante de la estructura de $\mathrm{BiFeO}_{3}$ [107] o el uso de $\mathrm{Tb}^{3+}$ en la estructura $\mathrm{Sr}_{2} \mathrm{MO}_{2}(\mathrm{M}=\mathrm{Sn}$ y $\mathrm{Zr})$ para dar un pigmento de coloración amarilla $[108]$.

Por lo que se refiere al uso de los lantánidos en la estructura tipo pirocloro/fluorita defectuosa como pigmentos cerámicos, no son muchas las investigaciones que se han realizado hasta el momento. Se puede resaltar el estudio realizado por Zhao y col. [109] desarrollando un pigmento cerámico de coloración amarilla, presentando una estructura pirocloro con una estequiometría $\mathrm{Y}_{2} \mathrm{Ce}_{2} \mathrm{O}_{7}$ dopado con $\mathrm{Fe}^{3+}$. También los estudios realizados por Martos y col. [104], desarrollando un pigmento de coloración rosa con una estructura pirocloro con estequiometría $\mathrm{Er}_{2} \mathrm{Ti}_{2} \mathrm{O}_{7}$. Otro estudio interesante, fue el realizado Stránská y col. [110], donde se abordó el estudio de las composiciones tipo $\mathrm{Ln}_{2} \mathrm{Zr}_{2} \mathrm{O}_{7}$ ( $\mathrm{Ln}=$ $\mathrm{Ho}, \mathrm{Er}, \mathrm{Yb}, \mathrm{Tm})$, donde el elemento lantánido jugó un papel fundamental en la posición A del pirocloro, determinando la coloración adoptada por el pigmento. En ningún caso se ha documentado hasta la fecha ningún sistema pigmentante basado en una estructura de fluorita defectuosa de itrio y circonio como red huésped.

En este sentido, en el siguiente apartado se abordará la potencial utilización del óxido mixto de itrio y circonio con estequiometría $\mathrm{Y}_{2} \mathrm{Zr}_{2} \mathrm{O}_{7}$, presentando una 
estructura de fluorita defectuosa, como red huésped de diferentes cationes para su potencial uso en el campo de los pigmentos cerámicos.

\subsection{5. $\mathrm{Y}_{2} \mathrm{Zr}_{2} \mathrm{O}_{7}$ como red huésped y sus potenciales aplicaciones}

En cuanto a la estructura fluorita defectuosa presentada por la estequiometría $\mathrm{Y}_{2} \mathrm{Zr}_{2} \mathrm{O}_{7}$, la cual nos atañe en la presente tesis, no existen en la bibliografía científica hasta el momento de la redacción, estudios con iones lantánidos como dopantes, a parte de las publicaciones generadas en la presente tesis, para aplicaciones en el campo de los pigmentos cerámicos.

En cambio, sí que se han realizado estudios en otros campos diferentes al de los pigmentos cerámicos. Compuestos con estequiometría $\mathrm{Y}_{2} \mathrm{Zr}_{2} \mathrm{O}_{7}$ han sido ampliamente utilizados como sistemas luminiscentes, donde los iones lantánidos dopan a la estructura, actuando como centros emisores. Claros son los ejemplos de $\mathrm{Y}_{2} \mathrm{Zr}_{2} \mathrm{O}_{7}$ dopado con iones lantánidos tales como, $\mathrm{Tb}^{3+}, \mathrm{Eu}^{3+}$ o $\mathrm{Dy}^{3+}$, presentando aplicaciones luminiscentes como fósforos [65,111-114]. Por lo tanto, los iones lantánidos por sus características y propiedades son óptimos para el dopaje de la estructura $\mathrm{Y}_{2} \mathrm{Zr}_{2} \mathrm{O}_{7}$. Además, parece interesante el codopaje de estructuras con iones lantánidos combinándolos con otro elemento dopante que actué como cromóforo como podría ser el hierro.

En la presente tesis se ha estudiado el uso de diferentes dopantes tanto en la posición del itrio como la del circonio. El itrio es sustituido parcialmente por diferentes elementos lantánidos (terbio y praseodimio), mientras que el circonio es sustituido en cantidades variables de hierro. Estos estudios han dado lugar a la publicación de dos artículos científicos en dos revistas internacionales de alto 
índice de impacto, tales como, Dyes and Pigmets y Journal of the European Ceramic Society.

En consecuencia, por todo lo mencionado en este punto, la producción de pigmentos respetables con el medio ambiente y multifuncionales, es de vital importancia en el campo de los pigmentos cerámicos actual, conllevando consigo un mayor valor añadido del producto final. Estos nuevos pigmentos serán mucho más atractivos y competitivos en comparación a los pigmentos cerámicos tradicionales teniendo una influencia directa en la calidad de vida en el siglo XXI, promoviendo un desarrollo sostenible y repercutiendo en una mejora de la calidad de vida actual, así como en la de las generaciones venideras.

En la presente tesis, poniendo de manifiesto la multifuncionalidad de estos óxidos mixtos, también se abordará el estudio de las propiedades de estos en un área bien diferente a la de los pigmentos cerámicos, como es la de las electrocerámicas. En el siguiente punto se revisarán, los antecedentes de estos óxidos mixtos en el campo de la electrocerámica, así como se explicarán algunos conceptos necesarios para el correcto entendimiento del trabajo realizado en la presente tesis.

\subsection{Electrocerámicas}

La electrocerámica es un término genérico que describe una clase de materiales inorgánicos, no metálicos, utilizados en la industria de la electrónica gracias a sus propiedades eléctricas, magnéticas u ópticas. El importante desarrollo en los dispositivos electrónicos que ha tenido lugar sobre todo en la segunda mitad del siglo XX, ha provocado un gran avance en los materiales cerámicos, siendo introducidos en una gran variedad de dispositivos y aplicaciones electrónicas. 
En este sentido, los óxidos mixtos pertenecientes al sistema $\mathrm{ZrO}_{2}-\mathrm{Y}_{2} \mathrm{O}_{3}$ en la zona rica en circonio, han sido ampliamente estudiados por sus buenas propiedades en el campo de la conducción iónica. No en vano, la circona estabilizada con un $\sim 8 \%$ molar de itria (YSZ08), es ampliamente utilizada como electrolito en celdas de combustible de óxido sólido de alta temperatura (SOFCs) o como sensor de oxígeno.

En el siguiente apartado se va a tratar en detalle el mecanismo de conducción mediante el cual el YSZ08 es capaz de conducir iones óxido a través de su estructura.

\subsubsection{Mecanismo de conducción iónica en YSZ}

Como se ha recalcado en el punto 1.3 del presente capítulo, es necesaria una estabilización de la estructura cúbica de la circona a temperatura ambiente para evitar problemáticas de cambios de fases y posibles agrietamientos. Este hecho es muy positivo, desde el punto de vista de un incremento en la conductividad iónica de la estructura, ya que está se verá incrementada debido al dopaje de la circona con cationes aliovalentes de menor valencia, en este caso la incorporación de $\mathrm{Y}^{3+}$, generando vacantes de oxígeno en la estructura cristalina, con el objetivo de mantener la electroneutralidad.

Usando la notación Kröger Vink [115], la sustitución de $\mathrm{Zr}^{4+}$ por $\mathrm{Y}^{3+}$, puede expresarse como:

$$
\mathrm{Y}_{2} \mathrm{O}_{3} \stackrel{\mathrm{ZrO}_{2}}{\longrightarrow} 2 \mathrm{Y}_{\mathrm{Zr}}^{\prime}+3 \mathrm{O}_{\mathrm{o}}^{\mathrm{x}}+\mathrm{V}_{\mathrm{o}}
$$

De este modo por cada ion $\mathrm{Y}^{3+}$ que se sustituye por un ion $\mathrm{Zr}^{4+}$, se generará una vacante de oxígeno por compensación de cargas, es decir una vacante por cada $\mathrm{Y}_{2} \mathrm{O}_{3}$ introducido en la estructura. Así, a mayor cantidad de catión estabilizador, en este caso el $\mathrm{Y}^{3+}$, se generarán más vacantes de oxígeno en la estructura, por lo que 
al tener más vacantes, el movimiento de los iones óxido debería ser mayor, comportando una menor energía de activación y una mayor conductividad iónica.

Sin embargo, esta tendencia descrita anteriormente, no se plasma experimentalmente, siendo necesaria una mínima cantidad de dopante para estabilizar la fase cúbica y obtener un máximo de conductividad. En el YSZ los valores de mínima energía de activación se dan con un dopaje del 8-9\% molar en $\mathrm{Y}_{2} \mathrm{O}_{3}$, generando una conductividad alrededor de $10^{-1}-10^{-2} \mathrm{~S} \cdot \mathrm{cm}^{-1}$ a $1000^{\circ} \mathrm{C}$ con unas energías de activación entre 0.8-0.9 eV [116].

Los estudios realizados a mayores concentraciones de dopante ponen de manifiesto un aumento de la energía de activación y una disminución de la conductividad iónica [117,118]. Dixon y col [118], han estudiado este fenómeno poniendo de manifiesto el aumento de la resistencia del material con el aumento de la cantidad de itria, como muestra el gráfico de Arrhenius de la figura 10.

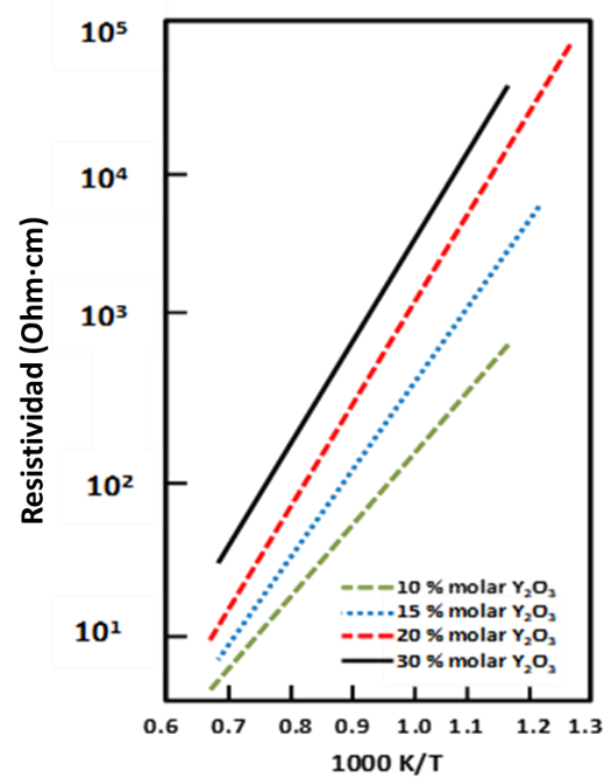

Figura 10. Gráfico de Arrhenius para diferentes composiciones del sistema $\mathrm{ZrO}_{2}-\mathrm{Y}_{2} \mathrm{O}_{3}$. (Adaptación de la referencia [118].) 
Como se observa, los valores de resistencia van aumentando progresivamente con el aumento de la concentración molar de óxido de itrio presente en la disolución sólida, adquiriendo valores máximos de resistencia la composición con mayor cantidad de itria, siendo está de un 30\% molar.

Una explicación bastante aceptada para esta tendencia, ha sido aportada por diversos autores, concluyendo a que es debida a la atracción de vacantes de oxígeno con el catión dopante, en este caso el itrio, formando especies complejas y disminuyendo la movilidad de los iones óxido [119]. El complejo formado presentará una carga positiva cuando la vacante de oxígeno se enlaza solamente con un catión dopante [120]:

$$
\mathrm{Y}_{\mathrm{Zr}}^{\prime}+\mathrm{V}_{\mathrm{o}} \leftrightarrow\left(\mathrm{Y}_{\mathrm{Zr}}^{\prime}-\mathrm{V}_{\mathrm{o}}\right)
$$

Un incremento mayor en la concentración de itrio y por tanto en la concentración de vacantes presentes en la estructura, conllevara una mayor formación de complejos itrio-vacante de oxígeno, formando los denominados "clusters".

$$
\mathrm{Y}_{\mathrm{Zr}}^{\prime}+\mathrm{V}_{\mathrm{o}}^{\ddot{4}} \leftrightarrow\left(\mathrm{Y}_{\mathrm{Zr}}^{\prime}-\mathrm{V}_{\mathrm{o}}-\mathrm{Y}_{\mathrm{Zr}}^{\prime}\right)^{\mathrm{x}}
$$

En dichos “clusters" la vacante de oxígeno está enlazada a dos cationes, disminuyendo en consecuencia la movilidad de las mismas, no teniendo la posibilidad de ser ocupada por un oxígeno, debido a la necesidad de una mayor cantidad de energía para mover el ion óxido a esta vacante, aumentando la energía de activación y disminuyendo la conducción iónica [119,121].

Sin embargo, existen fuertes evidencias, como se pondrá de manifiesto en la presente tesis, que análogamente a la formación de estos "clusters", responsables de una disminución de la conducción de iones óxido y el correspondiente aumento 
en la energía de activación del material, mayores concentraciones de itrio en la estructura, pueden favorecer la conducción electrónica frente a la conducción iónica. Una de las publicaciones derivadas de la presente tesis, sugiere la existencia de este comportamiento [122]. Por tanto, en el siguiente punto se van a exponer algunos antecedentes y conceptos de esta temática.

\subsubsection{Conducción electrónica en YSZ}

Los materiales basados en la disolución sólida circona estabilizada con un $8 \%$ molar de itria (YSZ08) se caracterizan por una conductividad alta de tipo iónico, marcada por la presencia de vacantes de oxígeno. A temperaturas de alrededor de $1.000{ }^{\circ} \mathrm{C}$, estos materiales son aptos para su aplicación como electrolitos sólidos en pilas de combustible, debido a la posibilidad de conducir iones $\mathrm{O}^{2-}$ a través de su estructura.

Dicha conducción de iones $\mathrm{O}^{2-}$ es independiente de las presiones parciales de oxígeno en un amplio intervalo de presiones. La conducción electrónica es tan pequeña respecto a la conducción de iones $\mathrm{O}^{2-}$ que se puede considerar despreciable en todo el dominio electrolítico. Schefold y col. [123] han determinado en el YSZ08, a una presión parcial de oxígeno de $10^{-11}$ atmósferas, el valor de la conducción electrónica, alcanzando valores mínimos de conductividad de alrededor de $10^{-8} \mathrm{~S} \cdot \mathrm{cm}^{-1}$, frente al valor de alrededor de $0.01 \mathrm{~S} \cdot \mathrm{cm}^{-1}$ correspondiente a la conducción iónica. Se considera que YSZ08 es estable en el dominio electrolítico por debajo de presiones parciales $10^{-20}$ atmósferas, hecho que permite su utilización como electrolito en contacto con el ánodo y con atmósferas reductoras. A mayores presiones parciales de oxígeno, no se conoce mucha información, ya que es difícil realizar medidas en intervalos superiores de presiones parciales de oxígeno a la presión atmosférica [122]. En el siguiente 
apartado se expone la dependencia del tipo de conducción predominante en el material en función de las presiones parciales de oxígeno.

\subsubsection{Mecanismos de conducción electrónica tipo n y p en electrocerámicas. Efecto de la atmósfera}

Como bien es conocido, los dominios electrolíticos e iónicos varían en función de las presiones parciales de oxígeno. La figura 11 muestra una representación esquemática general de los dominios iónicos y electrónicos, en función de la presión parcial de oxígeno [122].

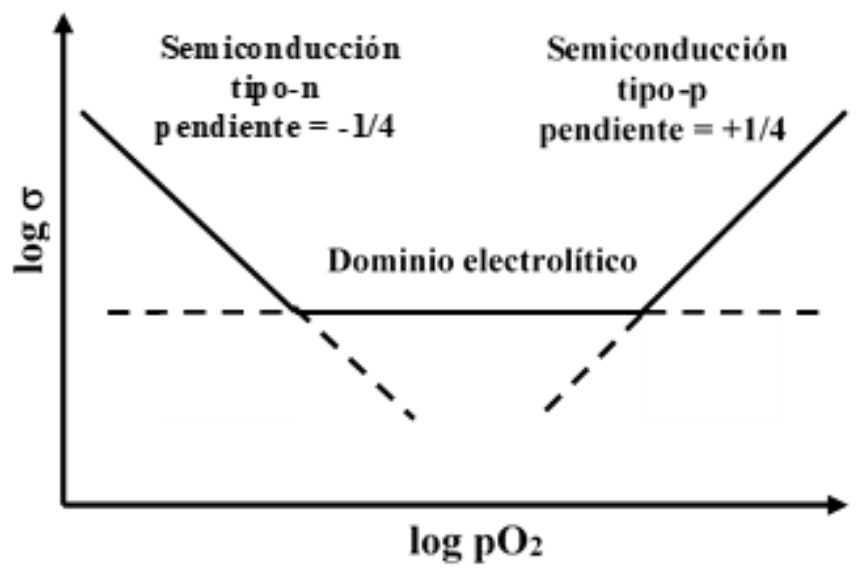

Figura 11. Representación esquemática de los dominós electrónicos y iónicos en función de la presión parcial de oxígeno [122].

Esta conducción iónica, en la zona del dominio electrolítico, está limitada por la zona de conducción electrónica tipo $n$ a presiones parciales de oxígeno bajas y por la zona de conducción electrónica tipo $p$ a presiones parciales de oxígeno altas.

A valores bajos de $\mathrm{pO}_{2}$ la conductividad aumenta con la pendiente, que adquiere un valor de $-1 / 4$ en una escala logarítmica y el material presenta una 
conducción tipo $n$. A mayores presiones parciales de oxígeno, donde la pendiente es 0 , la conducción es independiente de las presiones parciales del material, donde predomina la conducción iónica sobre la electrónica, esta zona se denomina dominio electrolítico.

A todavía mayores $\mathrm{pO}_{2}$, la conducción predominante es electrónica tipo $\mathrm{p}$, con una pendiente que adquiere un valor de $+1 / 4$ en escala logarítmica. En esta región la muestra absorbe oxígeno, ya sea en la superficie o por difusión en las vacantes aniónicas; los oxígenos absorbidos se disocian liberando electrones, tal y como muestra la ecuación 7:

$$
1 / 2 \mathrm{O}_{2}+\mathrm{V}_{\mathrm{o}}^{*}+2 \mathrm{e}^{\prime} \rightarrow \mathrm{O}_{\mathrm{o}}^{\mathrm{x}}
$$

De una forma alternativa y según la paridad electrón-hueco se puede reescribir también como:

$$
1 / 2 \mathrm{O}_{2}+\mathrm{V}_{\mathrm{o}} \rightarrow \mathrm{O}_{\mathrm{o}}^{\mathrm{x}}+2 h
$$

Cuando los huecos (h) creados en este proceso se convierten en las cargas dominantes, la conductividad será tipo $p$, como se muestra en la ecuación 8 . Sin embargo, si las cargas dominantes son los electrones creados, la conducción será tipo $n$, como muestra la ecuación 7 [124].

La conductividad de los materiales que son semiconductores extrínsecos tipo $n$ o $p$ es sensible a la atmósfera. En este sentido, con el objetivo de identificar el tipo de conducción de un material una buena estrategia es realizar medidas de impedancia en diferentes atmósferas, tales como nitrógeno, aire y oxígeno. En este caso y sin utilizar mezclas de gases, se pueden alcanzar presiones parciales de oxígeno de alrededor $10^{-2}$ atmósferas en mediciones realizadas en atmósfera de nitrógeno. Estas proporcionan una prueba simple para distinguir entre los diferentes mecanismos de conducción electrónica. 
Para los materiales que presentan una conducción tipo $n$, es decir donde las cargas dominantes son los electrones, al incrementar las presiones parciales de oxígeno, se induce a la absorción de oxígeno en la superficie de la muestra, lo que provocará una disminución de electrones en la misma. Si los electrones son los responsables de la conducción, al aumentar las presiones parciales de oxígeno la conductividad de la muestra disminuirá, presentando un mecanismo de conducción tipo $n$. El equilibrio asociado a esta absorción de moléculas de oxígeno en la superficie de la muestra se puede expresar como:

$$
\mathrm{O}_{2}+4 \mathrm{e}^{-} \leftrightarrow 20^{2-}
$$

Con los materiales que presentan una conducción tipo $p$ donde los portadores de carga mayoritarios son los huecos, el efecto que ocurre es justamente el contrario, los electrones que se capturan generan huecos, y por tanto un aumento de la presión parcial de oxígeno provoca el aumento de la conductividad. En este caso el equilibrio asociado vendrá dado por:

$$
\mathrm{O}_{2} \leftrightarrow 2 \mathrm{O}^{2-}+4 \mathrm{~h}^{+}
$$

Sin embargo, la ecuación 10 no especifica dónde se encuentran los huecos. Esta es una pregunta necesaria para entender el mecanismo de conducción, el cual dependerá del material a estudiar. Los huecos pueden ubicarse en los cationes si estos son capaces de oxidarse, o también en los iones óxido presentes en la estructura, si estos son capaces de ionizarse a un estado de valencia de menor carga [125].

Los equilibrios asociados idealmente con la absorción, disociación e ionización de las moléculas de oxígeno en las superficies de los materiales óxidos, son más complejos que los mostrados por las ecuaciones 9 y 10 . Los podemos representar de una forma más adecuada en la siguiente ecuación:

$$
\mathrm{O}_{2} \text { (g) } \stackrel{1}{\leftrightarrow} \mathrm{O}_{2} \text { (ads) } \stackrel{2}{\leftrightarrow} \mathrm{O}_{2}^{-} \stackrel{3}{\leftrightarrow} \mathrm{O}_{2}^{2-} / \mathrm{O}^{-} \stackrel{4}{\leftrightarrow} \mathrm{O}^{2-} \text { (superficie) } \stackrel{5}{\leftrightarrow} \mathrm{O}^{2-} \text { (bulk) }
$$


Los equilibrios 2, 3 y 4 de la ecuación 11 implican, la transferencia de electrones entre las especies de oxígeno que se originan a partir de la fase gaseosa y los electrones de la muestra. Los iones de la molécula de oxígeno, específicamente el peróxido $\left(\mathrm{O}_{2}{ }^{2-}\right)$ y el superóxido $\left(\mathrm{O}_{2}{ }^{-}\right)$, suelen estar presentes en la superficie, dependiendo de la temperatura y la atmósfera [126]. Normalmente, estas especies no tienen una influencia directa sobre las propiedades del "bulk", pero sí se producen desplazamientos en los equilibrios de la ecuación 11, provocando también cambios en la concentración de electrones presentes, afectando esto directamente a la conductividad. Si la muestra estudiada posee una elevada conductividad, estos pequeños cambios en la concentración de electrones o huecos, no se verán reflejados en la conductividad total. En cambio, si se está estudiando el efecto de la atmosfera en muestras aislantes o poco conductoras, este efecto será muy significativo afectando de una manera crítica los valores de la conducción, tal y como ocurre en semiconductores con comportamiento tipo $p$ o $n$ [124].

En este sentido, para estudiar dichos mecanismos, las medidas de espectroscopia de impedancia compleja (EI), a diferentes presiones parciales de oxígeno, proporcionan una ayuda fundamental para llegar a dilucidar el mecanismo de conducción predominante en un material determinado. Adicionalmente, la aplicación de voltajes externos ( $d c$ bias), puede proporcionar una gran información con el objetivo de dilucidar el mecanismo de conducción en electrocerámicas, como se argumentará en el siguiente punto. 


\subsubsection{Respuesta de un material electrocerámico a la aplicación de un voltaje externo}

Las medidas de impedancia no sólo pueden utilizarse para determinar el mecanismo de reacción a partir de los valores de resistencia en función de la presión parcial de oxígeno, sino también son útiles a la hora de identificar otro tipo de fenómenos a partir de la aplicación de un voltaje externo ( $d c$ bias). Los materiales cerámicos obedecen normalmente la ley de ohm, incluso cuando se les aplica voltajes pequeños. Esto es debido a que el gradiente de potencial que fluye a través de la muestra es demasiado pequeño para causar cambios estructurales, los cuales puedan afectar al tipo de conducción del material en cuestión. Como se ha visto a lo largo del presente capítulo, los materiales conducen electrónicamente por un mecanismo de saltos o bien iónico o electrónico. La aplicación de pequeños voltajes en corriente continua o $d c$ bias (direct current voltaje) simplemente aporta un flujo de carga en una dirección determinada, considerando que la conducción por saltos de iones, electrones o huecos no se ve influenciada al aplicar pequeños voltajes. Sin embargo, existen excepciones las cuales presentan una dependencia no lineal con la aplicación de un voltaje, no cumplen la ley de Ohm: materiales aislantes a los cuales se les aplican voltajes muy altos produciéndose la rotura dieléctrica del mismo [127], materiales cuya resistencia está controlada por efectos interfaciales entre la muestra y el electrodo como son las barreras de Scotttky [128] y materiales cuya resistencia depende de la magnitud y la polaridad del voltaje, como los memristores [129]. En este sentido diversos autores han demostrado el sorprendente comportamiento que presentan algunas electrocerámicas bajo la aplicación de un voltaje $d c$, mostrando una influencia directa en la conductividad del "bulk”[125,130,131]. 
Por lo tanto, la espectroscopia de impedancia combinada con la aplicación de pequeños voltajes $d c$, permite diferenciar entre fenómenos interfaciales y fenómenos de la propia muestra; ya que la técnica permite separar la contribución del bulk de los efectos interfaciales como las barreras de Scthottky. Estas barreras están asociadas a la diferencia entre los niveles de energía de Fermi de dos materiales en contacto, como pueden ser la muestra y el electrodo, un material de naturaleza aislante o semiconductora y un metal respectivamente. En la figura 12a se muestra la estructura de bandas para un metal y un semiconductor, en este caso tipo $n$, antes de producirse el contacto entre ambos. Cuando se produce el contacto, considerando una unión ideal, los niveles de fermi tienden a igualarse, provocando una difusión de electrones del semiconductor hacia el metal, provocando en consecuencia una región de carga espacial positiva en el semiconductor (de anchura d), tal y como muestra la figura $12 \mathrm{~b}$.

Por lo tanto, este fenómeno conlleva un cúmulo de carga en un lado de la interfase, la cual induce una polarización al otro lado, oponiéndose al movimiento de los electrones desde el metal hacia el semiconductor y en sentido opuesto. En esta situación el flujo de corriente a través del material, presenta una resistencia no lineal, ya que la corriente fluye con mayor facilidad en un sentido, del semiconductor al metal, que en el opuesto. La figura 12c, muestra de una forma esquemática la interfase formada (“depleted región”) al producirse las barreras de Schottky.

Este fenómeno no es exclusivo del contacto electrodo muestra, sino que también se puede dar en las fronteras de grano. En general, una muestra está compuesta por diferentes granos, los cuales pueden presentar estructuras electrónicas diferentes, presentando diferencias en sus niveles de Fermi, si bien es cierto, las diferencias entre los niveles serán más similares que las mostradas en la figura 12 para la interfase metal-semiconductor, produciéndose barreras de potencial mucho más pequeñas. Con el objetivo de mantener el equilibrio 
termodinámico, los electrones pueden fluir por las fronteras de grano quedando atrapados por los defectos del material o los posibles dopantes, aumentando los niveles de Fermi, hasta que se igualen en todo el material. Como consecuencia de dicho comportamiento se forman capas de carga negativa en los bordes de grano, las cuales son neutralizadas por sitios dadores de carga positiva en ambos lados del borde de grano, generando una región de carga espacial o de agotamiento (“depleted región”).
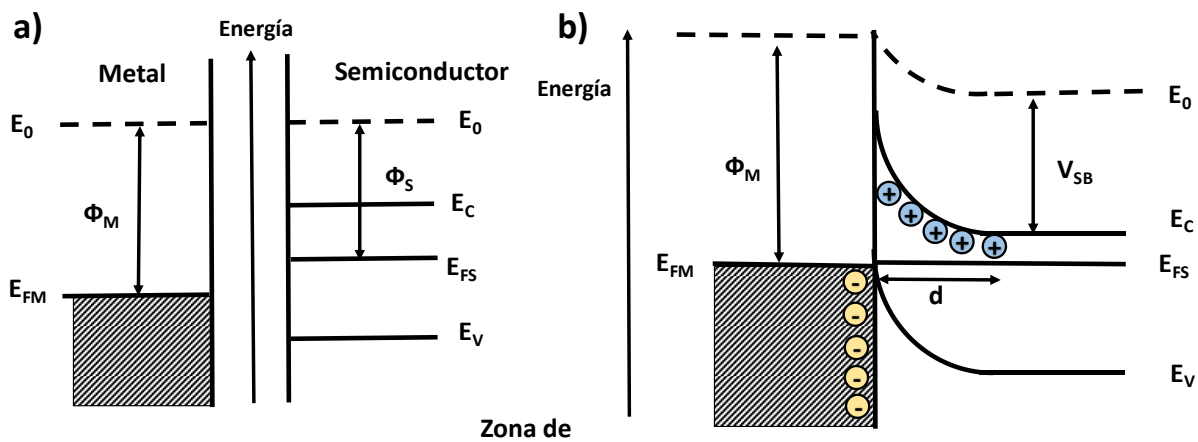

c)

depleción Semiconductor

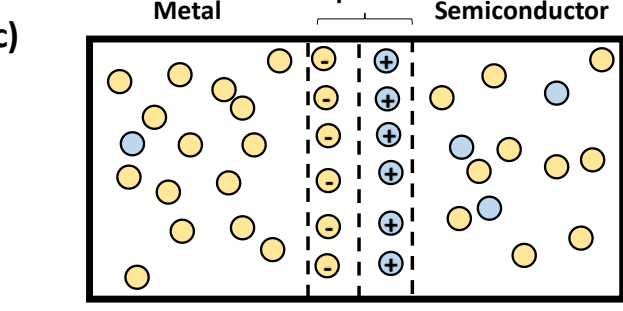

Figura 12. (a) Diagrama de bandas para un metal y un semiconductor tipo $\mathrm{n}$ antes del contacto. (b) Diagrama de bandas para un metal y un semiconductor tipo n en contacto, donde los niveles de Fermi de ambos se igualan, formando la barrera de Schottky por inyección de electrones del semiconductor al metal. (c) Esquema de una barrera de Schottky, observándose la "depleted región", presentando un comportamiento muy similar a una unión pn. Donde $\mathrm{E}_{0}$ es el nivel de vacío, $\mathrm{E}_{\mathrm{FM}} \mathrm{y} \mathrm{E}_{\mathrm{FS}}$ son los niveles de fermi del metal y el semiconductor respectivamente, $\mathrm{E}_{\mathrm{C}}$ es la banda de conducción y $\mathrm{E}_{\mathrm{V}}$ la banda de valencia. $\Phi_{\mathrm{M}} \Phi_{\mathrm{S}}$ son las funciones trabajo del metal y del semiconductor respectivamente . 
De esta forma se genera una doble barrera de Schottky en el borde de grano, la cual tendrá una influencia directa en las propiedades físicas que presenta el material. Los electrones necesitaran poseer una energía determinada para vencer esta barrera de potencial que se ha creado. Sólo unos pocos electrones serán capaces de superarla en ausencia de un potencial externo. Sin embargo, la introducción de un potencial externo ( $d c$ bias) proporcionará una energía adicional a estos electrones, los cuales serán capaces de superar esta barrera y alcanzar otro grano. Por lo tanto, la espectroscopia de impedancia combinada con la aplicación de un voltaje externo ( $d c$ bias), es capaz de variar la conducción a través de las interfases modificando dicha barrera, provocando una dependencia de la resistencia en función del voltaje. Así la aplicación de pequeños $d c$ bias, ha contribuido a entender en detalle las diferentes regiones de la muestra. De esta forma, como se verá a continuación, realizando medidas de impedancia junto a la aplicación de pequeños voltajes se han podido entender ciertos fenómenos difícilmente entendibles sin el uso de esta herramienta, por ejemplo los fenómenos observados al aplicar pequeños voltajes en electrocerámicas dopadas con dopantes aceptores.

\section{Aplicación de un voltaje en electrocerámicas dopadas con iones aceptores}

Diversos autores han realizado estudios del efecto de la aplicación de pequeños voltajes en electrocerámicas dopada con iones aceptores, como el $\mathrm{BaTiO}_{3}$ con dopantes tales como, magnesio, calcio y zinc [125,130,131]. El efecto del dopaje con estos iones divalentes sustituyendo al $\mathrm{Ti}^{4+}$ conlleva consigo la creación de una vacante de oxígeno por cada ion divalente sustituido, manteniendo la electroneutralidad estructural. En estos estudios se realizaron medidas de impedancia combinadas con la aplicación de diferentes voltajes, con el objetivo de estudiar el comportamiento del bulk y de la frontera de grano, para distinguir posibles resistencias asociadas a efectos interfaciales, de las asociadas a las de la 
propia muestra. En ambos casos la aplicación del voltaje conllevó una disminución de la resistencia. La resistencia de la frontera de grano disminuyó al aumentar el voltaje aplicado. Este comportamiento es el esperado para un fenómeno interfacial asociado a una diferencia en los niveles de Fermi. Sin embargo, la disminución de la resistencia en el bulk fue un resultado inesperado, mostrando una clara dependencia tanto con el tiempo de aplicación del $d c$ bias, como de la magnitud del voltaje aplicado [125]. Con la supresión del voltaje durante un tiempo suficiente, la muestra adquiría nuevamente sus valores iniciales. Este incremento de hasta de dos órdenes de magnitud en la conductividad del bulk, nunca había sido observado anteriormente.

Adicionalmente, este efecto se veía incrementado con el aumento de la cantidad de dopante. Los resultados obtenidos de las medidas realizadas en diferentes atmósferas, tanto en estado fundamental, como en el estado excitado (con aplicación de $d c$ bias), muestran para ambos casos un mecanismo de conducción tipo $p$, es decir donde las cargas dominantes son los huecos. ¿A qué es debido este aumento tan significativo en la conductividad con las aplicaciones de pequeños voltajes $d c$ ? Los autores plantearon una respuesta a dicha pregunta.

En esta estructura, ninguno de los componentes presentes en ella, puede ser oxidado generando los huecos necesarios para la conducción tipo $p$. Por lo tanto, la única fuente de huecos debe de recaer en una posible ionización de los iones $\mathrm{O}^{2-}$, coordinados con los metales en la estructura. El ion $\mathrm{O}^{2-}$ se estabiliza en estado sólido sólo como consecuencia de la energía reticular extra asociada con los iones óxido doblemente cargados; el ion óxido es termodinámicamente inestable en fase gas como resultado de la segunda afinidad electrónica presentada para la reacción $\mathrm{O}^{-}+\mathrm{e}^{-} \rightarrow \mathrm{O}^{2-}$, que es positiva. Sin embargo, bajo determinadas condiciones un ion $\mathrm{O}^{2-}$, puede desestabilizarse en el retículo cristalino y en consecuencia ionizarse fácilmente para dar el ion $\mathrm{O}^{-}[125,126,130,131]$. 
En las muestras donde la estructura se dopa con iones aceptores, se generan vacantes de oxígeno. En el caso del $\mathrm{BaTiO}_{3}$ dopado con iones divalentes, estos se encontrarán rodeados de cinco iones $\mathrm{O}^{2-}$ y una vacante de oxígeno. Los iones óxido adyacentes $\left(\mathrm{O}_{2}\right)$ estarán débilmente enlazados como consecuencia de la carga del ion divalente, dos veces menor que el $\mathrm{Ti}^{4+}$ en este caso. Bajo estas condiciones, estos iones $\mathrm{O}^{2-}$, absorbidos tal y como muestra la ecuación 12, pueden ionizarse según:

$$
\mathrm{O}^{2-} \rightarrow \mathrm{O}^{-}+\mathrm{e}^{-}
$$

Los iones $\mathrm{O}^{-}$resultantes constituyen la fuente de huecos y los electrones liberados probablemente ocupen a los orbitales $3 \mathrm{~d}$ del metal, en este caso el titanio.

Esta generación de $\mathrm{O}^{-}$ha sido asociada a la conducción p en atmósferas parciales de oxígeno altas, así como en la aplicación de pequeños voltajes. Estos comportamientos, han sido comprobados en diversas estructuras aceptoras tales como, $\mathrm{Ba}\left(\mathrm{Ti}_{1-\mathrm{x}} \mathrm{Mg}_{\mathrm{x}}\right) \mathrm{O}_{3-\mathrm{x}}, \mathrm{Ba}\left(\mathrm{Ti}_{1-\mathrm{x}} \mathrm{Ca}_{\mathrm{x}}\right) \mathrm{O}_{3-\mathrm{x}}, \mathrm{Ba}\left(\mathrm{Ti}_{1-\mathrm{x}} \mathrm{Zn}_{\mathrm{x}}\right) \mathrm{O}_{3-\mathrm{x}}, \quad \mathrm{Sr}\left(\mathrm{Ti}_{1-\mathrm{x}} \mathrm{Mg}_{\mathrm{x}}\right) \mathrm{O}_{3-\mathrm{x}}, \quad \mathrm{y}$ $\left(\mathrm{Bi}_{1-\mathrm{x}} \mathrm{Ca}_{\mathrm{x}}\right) \mathrm{FeO}_{3-\mathrm{x} / 2}[125,126,130-133]$.

El aumento de la conductividad, en estas estructuras es debido a la ionización de los $\mathrm{O}_{2}$ débilmente enlazados (debido a las vacantes generadas en la estructura), generando $\mathrm{O}^{-}$. Los electrones generados son "atrapados" en el equilibrio propuesto en la ecuación 11, actuando como fuerza motriz, permitiendo la generación de más iones $\mathrm{O}^{-}$y en consecuencia de huecos, repercutiendo en un aumento de la conductividad del bulk y en consecuencia del material.

Además, este modelo de conductividad junto con la realización de medidas impedancia aplicando diferentes voltajes, puede ayudar a entender la disminución de la resistencia asociada al bulk, no sólo para el $\mathrm{BaTiO}_{3}$, sino también para otros materiales dopados con iones aceptores como puede ser el caso del YSZ08. 


\section{Aplicación de pequeños voltajes en YSZ08}

La estructura YSZ08, presenta una estructura cúbica, debido a la estabilización de la circona por el dopaje con iones $\mathrm{Y}^{3+}$, creando vacantes de oxígeno las cuales estabilizan la estructura. Estas vacantes, además de estabilizar la estructura cúbica, le proporcionan una elevada conducción iónica, siendo la conducción electrónica en esta composición despreciable a temperaturas intermedias [134-136]. Steele y col [136], han demostrado la existencia de una elevada conductividad iónica, en un amplio rango de presiones parciales de oxígeno, lo cual indica que la muestra se encuentra en el dominio electrolítico, siendo la conducción fundamentalmente iónica, presentando una conducción electrónica despreciable $[123,136]$. No en vano, se ha documentado que a $800^{\circ} \mathrm{C}$ la conducción electrónica se iguala a la conducción iónica a un valor de presión parcial de $\mathrm{pO}_{2} \sim 10^{-38}$ bar [123].

Con el objetivo de estudiar el comportamiento eléctrico del YSZ08, Masó y West [137] estudiaron el efecto de la aplicación de pequeños voltajes a diferentes temperaturas en atmósfera de aire, obteniendo resultados sorprendentes. Igual que en los casos mencionados anteriormente, se observó una disminución en los valores de la resistencia asociada al bulk a bajas temperaturas, análogamente a lo acontecido en las estructuras $\mathrm{BaTiO}_{3}$ con dopantes aceptores. Al eliminar la aplicación de dicho voltaje, la muestra recuperaba sus valores iníciales rápidamente. Adicionalmente, a esta variación en la conductividad del bulk, se observó también un colapso en el "spike" a bajas frecuencias. Este hecho sugiere la posible introducción de la conducción electrónica en la muestra [138,139].

Con el objetivo de comprobar estas evidencias, los autores realizaron un ajuste teórico de las medidas de impedancia ("fitting"), para la muestra con voltaje y sin voltaje. La figura 13 muestra, los circuitos propuestos, así como los datos experimentales comparados con los simulados mediante circuitos teóricos. 
En la figura 13 se observa el efecto de la aplicación de un voltaje en YSZ08. Se ha comentado, que al aplicar el voltaje se aprecia una disminución de la resistencia, junto a un colapso del "spike" a bajas frecuencias tal y como se observa comparando la Figura 13c y d.

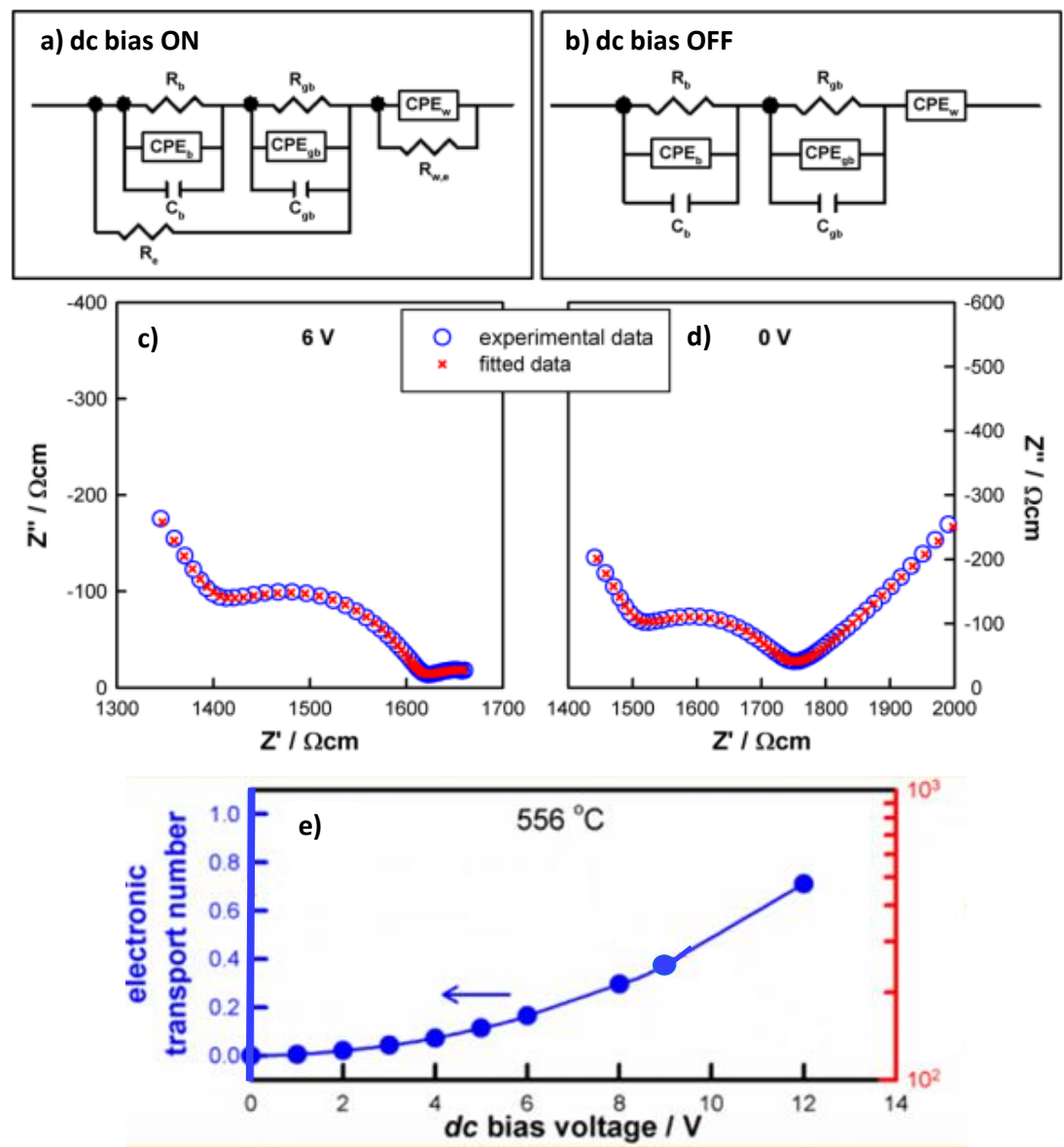

Figura 13. Circuitos equivalentes teóricos usados como modelo (a y b); medidas de impedancia $Z^{*}$ experimentales (azul) y simulación teórica (rojo) (c y d); transporte electrónico en función del voltaje aplicado (f). (Adaptación de la referencia [137].)

Este hecho es debido a la introducción de la conducción electrónica en el circuito. Si comparamos la figura 13 a y b, ambas tienen los mismos componentes, la única diferencia es la introducción de una resistencia en paralelo asociada a la conducción electrónica cuando el voltaje está aplicado. Además, podemos observar 
que las simulaciones teóricas son muy similares a las impedancias obtenidas experimentalmente, como muestra la figura $13 \mathrm{c}$ y d. Además, otra clara evidencia de la introducción de la conducción electrónica con la aplicación de voltajes se muestra en la figura 13e, donde los autores han representado el número de transporte electrónico, en función del voltaje aplicado. Esta figura muestra claramente el aumento del transporte electrónico, con el aumento del voltaje, siendo una clara evidencia de la introducción electrónica en una muestra puramente iónica.

Como se ha visto anteriormente en composiciones con dopantes aceptores [125,126,131-133], la generación de vacantes de oxígeno está íntimamente ligada con la generación de huecos, los cuales son los responsables de la conducción, en este caso tipo $p$.

En estas composiciones, donde ninguno de sus elementos es susceptible de reducirse, la generación de dichos huecos debe de recaer en los oxígenos, generando especies $\mathrm{O}^{-}$.Este mecanismo explica la introducción de la conducción electrónica tipo $p$ en paralelo en el YSZ08, mediante la aplicación de pequeños voltajes, ya que permite una mayor ionización de los oxígenos débilmente enlazados, debido a la formación de vacantes de oxígeno, al mismo tiempo que proporciona electrones al equilibrio descrito en la ecuación 11, hecho que favoreceré aún más si cabe, la creación de huecos y por lo tanto la conducción tipo $p$.

Este comportamiento puede estar íntimamente ligado a la cantidad de ion estabilizador, en este caso el itrio, presente en la estructura, ya que puede permitir una mayor ionización de los $\mathrm{O}^{-}$tal y como se describe en la ecuación 12, debido a una mayor presencia de $\mathrm{O}_{2}$ débilmente enlazados, presentando una influencia directa en los valores de conductividad del bulk y en consecuencia del material. 
Por lo tanto, en la presente tesis se va a estudiar la existencia de este comportamiento en diferentes composiciones de $\mathrm{ZrO}_{2}-\mathrm{Y}_{2} \mathrm{O}_{3}$, más concretamente a cantidades superiores al $8 \%$ molar en itria, como se verá de una forma detallada en el capítulo 5. 



\section{CAPÍTULO 2}

\section{Objetivos}

"Lo que sabemos es una gota de agua; lo que ignoramos es el océano" Issac Newton 



\section{Objetivos generales}

Como se ha visto, la estequiometría $\mathrm{Y}_{2} \mathrm{Zr}_{2} \mathrm{O}_{7}$ es capaz de albergar una gran diversidad de diferentes elementos en su estructura. Esta posibilidad hace que dicha estequiometría, sea potencialmente útil en una gran variedad de aplicaciones. En este sentido, la presente tesis está centrada en dos campos diferentes de la ciencia, como son el campo de los pigmentos cerámicos y el campo de la electrocerámica.

Los objetivos generales de la presente tesis en el ámbito de los pigmentos cerámicos son los siguientes:

- Diseñar la síntesis de nuevos sistemas pigmentantes de coloración rojiza respetables con el medioambiente, basados en la estequiometría $\mathrm{Y}_{2} \mathrm{Zr}_{2} \mathrm{O}_{7}$ dopados con diferentes iones lantánidos, usando el hierro como elemento cromóforo.

- Caracterizar estructural y microestructuralmente los pigmentos cerámicos obtenidos en polvo.

- Estudiar la estabilidad y caracterizar ópticamente las estructuras pigmentantes en diferentes medios, tales como esmaltes cerámicos, polímeros y pinturas.

- Estudiar los mecanismos de coloración a través del seguimiento de los estados de oxidación de los cromóforos, antes y después de su incorporación en el esmalte.

- Estudiar las propiedades proporcionadas por el pigmento al incorporarse a los diferentes medios para su aplicación como "cool pigment". 
Como se ha expuesto el sistema $\mathrm{ZrO}_{2}-\mathrm{Y}_{2} \mathrm{O}_{3}$, es ampliamente utilizado también en el campo de la electrocerámica por sus propiedades en el campo de la conducción de ion óxido, sobre todo en la zona rica en circonio.

Sin embargo, los estudios realizados en zonas más ricas en $\mathrm{Y}_{2} \mathrm{O}_{3}$, son muy pobres, ya que se ha comprobado una reducción de la conducción iónica con el aumento de contenido en itrio. No existen a mayores composiciones al $30 \%$ molar en itria, estudios de caracterización eléctrica con cambio de atmósfera o con aplicación de pequeños voltajes $d c$. Por lo tanto, es interesante la realización de un estudio, en el cual se podrían establecer los siguientes objetivos generales:

- Sintetizar mediante el método sol-gel polimérico disoluciones sólidas del sistema $\mathrm{ZrO}_{2}-\mathrm{Y}_{2} \mathrm{O}_{3}$ con composiciones molares de $\mathrm{Y}_{2} \mathrm{O}_{3}$ superiores al $30 \%$.

- Estudiar el efecto en las propiedades eléctricas de la variación en la cantidad molar de itrio presente en la disolución sólida.

- Estudiar el efecto en las propiedades eléctricas del cambio de atmósfera en las diferentes composiciones del sistema $\mathrm{ZrO}_{2}-\mathrm{Y}_{2} \mathrm{O}_{3}$.

- Estudiar el efecto en las propiedades eléctricas de la aplicación de un voltaje $d c$ en las diferentes composiciones del sistema $\mathrm{ZrO}_{2}-\mathrm{Y}_{2} \mathrm{O}_{3}$.

- Comparar los resultados obtenidos con aquellos obtenidos para el $\mathrm{ZrO}_{2}$ dopado con un $8 \%$ molar en $\mathrm{Y}_{2} \mathrm{O}_{3}$. 


\section{CAPÍTULO 3}

\section{Síntesis del pigmento multifuncional basado en $\mathrm{Y}_{2} \mathrm{Zr}_{2} \mathrm{O}_{7}$ dopado con $\mathrm{Tb}$ y $\mathrm{Fe}$}

"El peligro radica en que nuestro poder para dañar o destruir el medio ambiente o a nuestros pares, aumenta a mucha mayor velocidad que nuestra sabiduría en el uso de ese poder" Stephen Hawkings 



\subsection{Introducción}

En el presente capítulo, se aborda la síntesis de un nuevo pigmento cerámico basado en una estructura fluorita defectuosa con estequiometría $\mathrm{Y}_{2} \mathrm{Zr}_{2} \mathrm{O}_{7}$ dopado con terbio en la posición del itrio y con hierro en la posición del circonio.

El terbio es uno de los lantánidos más utilizados en el campo de los pigmentos cerámicos, debido a su configuración electrónica [108,140-142]. Al igual que el resto de los lantánidos, el terbio presenta la suma de las tres entalpias de reacción relativamente baja, por lo que es fuertemente electropositivo, formando con facilidad iones +3 (configuración $4 f^{8}$ ), si bien es cierto que también se puede presentar en estado de oxidación +4 (configuración $4 f^{7}$ ). El terbio en estado de oxidación +3 , presenta propiedades fotoluminiscentes, las cuales dependen fuertemente de las propiedades físicas y químicas de la red que los aloja, así como de su estructura y dimensiones [143]. Existen investigaciones en las cuales el $\mathrm{Y}_{2} \mathrm{Zr}_{2} \mathrm{O}_{7}$ se dopa con iones $\mathrm{Tb}^{3+}$ para aplicaciones fotoluminiscentes como fósforos [65]. Esta fotoluminiscencia es debida a transiciones entre los orbitales $f$, como se observa en la figura 14, donde se muestra esquemáticamente mediante el diagrama de energía, el mecanismo de emisión para el $\mathrm{Tb}^{3+}[144]$.

En la figura 14, se observa la promoción de un electrón del nivel fundamental $4 f$ a un estado excitado localizado en el orbital $5 d$ bajo una excitación óptica. El electrón excitado se relaja no radiactivamente hasta el nivel ${ }^{5} \mathrm{D}_{3}$ del

orbital $f$. La transición ${ }^{5} \mathrm{D}_{3} \rightarrow{ }^{5} \mathrm{D}_{4}$ tiene lugar por un proceso de relajación no radiativa [145]. Las transiciones radiativas ${ }^{5} \mathrm{D}_{4} \rightarrow{ }^{7} \mathrm{~F}_{\mathrm{n}=3,4,5,6}$ con longitudes de onda en la zona verde del espectro visible, producen una luminiscencia de dicha coloración, siendo la más intensa la transición ${ }^{5} \mathrm{D}_{4} \rightarrow{ }^{7} \mathrm{~F}_{5}$. 


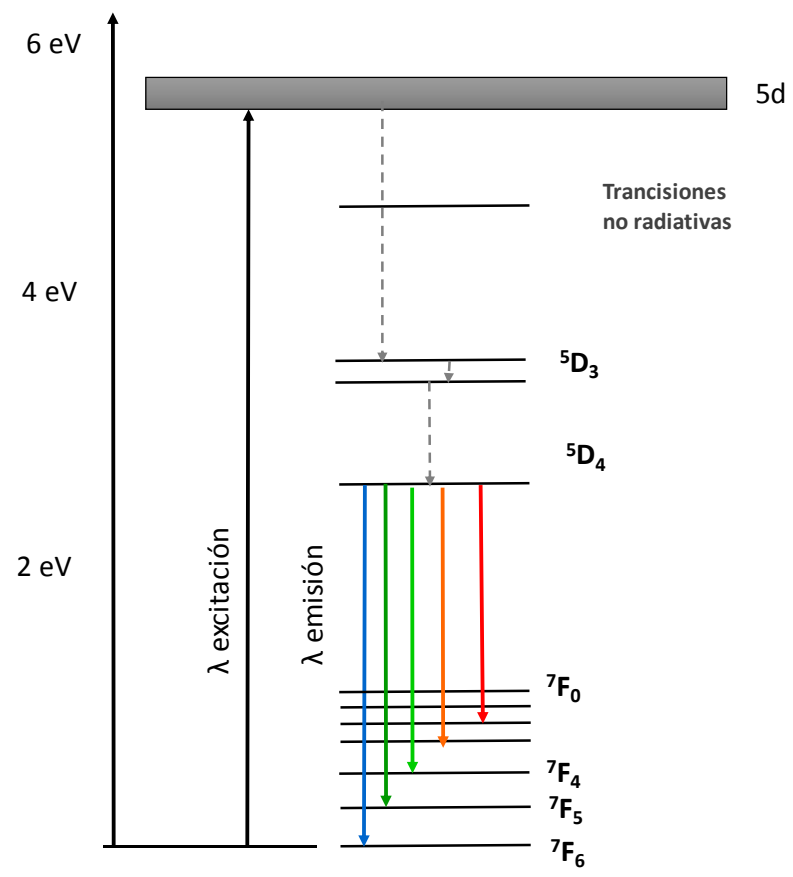

Figura 14. Diagrama de niveles energéticos y transiciones del $\mathrm{Tb}^{3+}$.

Este fenómeno ocurre exclusivamente cuando el estado de oxidación del terbio es +3 , en cambio no sucede cuando su estado de oxidación es +4 . Esto es debido al fenómeno denominado auto-ionización seguido de una transición no radiativa inhibiendo la transición ${ }^{5} \mathrm{D}_{3} \rightarrow{ }^{7} \mathrm{~F}_{\mathrm{j}}$ cuando el estado de oxidación es +4 $[146,147]$.

El conocimiento de los mecanismos de coloración en los pigmentos cerámicos es fundamental para desarrollar nuevos pigmentos cerámicos, pudiendo optimizar la síntesis, modificando los parámetros de reacción con el objetivo de favorecer el desarrollo del color final deseado. En este sentido el comportamiento del terbio puede ser de gran ayuda en el estudio de los mecanismos de coloración en el campo de los pigmentos cerámicos, donde el estado de oxidación de los elementos tiene una fuerte influencia en el color del 
producto final. Un claro ejemplo de la influencia en el color final del estado de oxidación de los pigmentos cerámicos, se pone de manifiesto en el estudio realizado por De la Luz y col. [105]. En este estudio se prepararon disoluciones sólidas de $\mathrm{Y}_{2} \mathrm{O}_{3}$ codopadas con terbio y calcio o bario, corroborando que la coloración amarilla-naranja del pigmento cerámico final estaba relacionada principalmente con la presencia de $\mathrm{Tb}^{4+}$. Esto se asocia al hecho de que el terbio en estado de oxidación +4 presenta una transferencia de carga, atribuida a $\mathrm{Tb}^{4+} \rightarrow \mathrm{O}^{2-}$ en la región de azul-verde del espectro visible $(400-550 \mathrm{~nm})$, intensificando por tanto la coloración amarillenta, ya que es reflejada por el pigmento, proporcionándole su color final $[105,144,148]$. Por tanto, tal y como muestra este trabajo, los diferentes estados de oxidación del terbio tienen una fuerte influencia en la coloración final presentada por el pigmento.

Por otra parte, como se ha expuesto en el capítulo 1, el hierro es uno de los cromóforos íntimamente relacionados con el desarrollo de una coloración rojiza en su estado de oxidación +3 .

Por lo anteriormente expuesto, en el presente estudio se realizó una síntesis mediante el método sol-gel de las composiciones $\mathrm{Y}_{2-\mathrm{x}} \mathrm{Tb}_{\mathrm{x}} \mathrm{Zr}_{2-\mathrm{y}} \mathrm{Fe}_{\mathrm{y}} \mathrm{O}_{7-\delta}$ donde " $\mathrm{x}$ " presenta un valor de 0 o 0.25 y la " $y$ " valores variables $0.15,0.25 \mathrm{y}$ 0.35. La elección del terbio como ion dopante, fue debida a las propiedades ópticas descritas en los párrafos anteriores. Además, se realizó un estudio detallado de la coloración tanto en las muestras en polvo como en las muestras aplicadas a un esmalte y se compararon con la del pigmento rojo usado industrialmente, el pink coral. Posteriormente se relacionó la coloración con los estados de oxidación de los cromóforos y además se estudió detalladamente el papel que juega el terbio en la disolución sólida. 
Adicionalmente, se ampliaron aplicaciones del pigmento preparado como "cool pigment" tanto en esmalte cerámico como en pintura.

En el siguiente punto se definen los objetivos que se plantearon para dicho estudio. 


\subsection{Objetivos del artículo científico 1}

Los objetivos generales del presente trabajo son:

- Optimizar los parámetros en la síntesis vía sol-gel polimérico, de un pigmento cerámico de coloración rojiza basado en la composición con estequiometría $\mathrm{Y}_{2} \mathrm{Zr}_{2} \mathrm{O}_{7}$ dopado con hierro y terbio.

- Establecer el límite de solubilidad para las posibles disoluciones sólidas con estequiometria, $\mathrm{Y}_{2} \mathrm{Zr}_{2-\mathrm{y}} \mathrm{Fe}_{\mathrm{y}} \mathrm{O}_{7-\delta}$ y $\mathrm{Y}_{1.75} \mathrm{~Tb}_{0.25} \mathrm{Zr}_{2-\mathrm{y}} \mathrm{Fe}_{\mathrm{y}} \mathrm{O}_{7-\delta}$ adquiriendo "y" valores de $0.15,0.25,0.35$.

- Estudiar el papel del terbio en la estructura y la influencia de este en la formación de disoluciones sólidas.

- Estudiar los estados de oxidación de los iones dopantes, así como su posible cambio al incorporarse al esmalte y relacionarlos con la coloración desarrollada en el producto final.

- Comprobar la estabilidad del pigmento en un esmalte cerámico y el desarrollo del color que se produce en el mismo.

- Comprobar el potencial uso del pigmento en el área de los pigmentos fríos o "cool pigments", en diferentes medios de dispersión, tales como el propio esmalte o al mezclarlo con una pintura. 


\subsection{Artículo científico 1}

Título: New red-shade environmental-friendly multifunctional pigment based on $\mathrm{Tb}$ and $\mathrm{Fe}$ doped $\mathrm{Y}_{2} \mathrm{Zr}_{2} \mathrm{O}_{7}$ forceramicapplications and cool roof coatings

Autores: Marc Jovaní, Ana Sanz, Héctor Beltrán-Mir, Eloisa Cordoncillo

Revista: Dyes and Pigments

Índice de Impacto: 3.473

Revista dentro del $25 \%$ : $\mathrm{Si}$

Categoría: Química Aplicada

Posición: 11/72 


\title{
New red-shade environmental-friendly multifunctional pigment based on $\mathrm{Tb}$ and Fe doped $\mathrm{Y}_{2} \mathrm{Zr}_{2} \mathrm{O}_{7}$ for ceramic applications and cool roof coatings
}

\author{
Marc Jovaní, Ana Sanz, Héctor Beltrán-Mir, Eloisa Cordoncillo \\ Departamento de Química Inorgánica y Orgánica, Universitat Jaume I de Castellón, Avda. Sos Baynat s/n, 12071, Castellón de la Plana, Spain
}

\section{A R T I C L E I N F O}

\section{Article history:}

Received 26 February 2016

Received in revised form

16 May 2016

Accepted 22 May 2016

Available online 24 May 2016

\section{Keywords:}

Red pigment

Sol-gel

NIR reflectance

Terbium

Defect fluorite

\begin{abstract}
A B S T R A C T
A new red-shade environmental friendly ceramic pigment with the general formula $\mathrm{Y}_{2-\mathrm{x}} \mathrm{Tb}_{\mathrm{x}} \mathrm{Zr}_{2-\mathrm{y}} \mathrm{Fe}_{\mathrm{y}} \mathrm{O}_{7-\delta}$ $[\mathrm{x}=0,0.25$ and $\mathrm{y}=0.15,0.25,0.35]$ was prepared by a polymeric sol-gel process and after calcination at $1300{ }^{\circ} \mathrm{C}$ for $12 \mathrm{~h}$. The solid solution range with defect fluorite structure was determined by XRD. The presence of terbium ions promoted the incorporation of iron ions in the structure. The evolution of the colour was studied by UV/Vis absorption measurements and CIELab chromatic coordinates were determined. The colour was related to the oxidation states of the terbium and iron ions studied by XPS and photoluminescence. Pigments presented the best chromatic coordinates when the amount of $\mathrm{Fe}(\mathrm{III})$ and $\mathrm{Tb}(\mathrm{IV})$ ions were higher. The sample with good red colouration $(\mathrm{x}=0.25, \mathrm{y}=0.35)$ was tested with an industrial frit, presenting slightly better chromatic coordinates to those of the commercial ceramic pigment currently used. The pigment also showed an optimal NIR reflectance to be applied as "cool pigment" for roof isolation.
\end{abstract}

๑) 2016 Published by Elsevier Ltd.

\section{Introduction}

Compounds with $\mathrm{A}_{2} \mathrm{~B}_{2} \mathrm{O}_{7}$ stoichiometry have been extensively studied for their extensive field of applications and also for their ability to incorporate a wide range of chromophore ions in both position A and B [1,2]. This structure is optimal in order to develop pure colours due to the possibility of incorporating a great range of chromophores. The research of new ceramic pigments is a high priority field. One of the biggest current challenges is the preparation of a new red-shade ceramic pigment, due to the scarce variety of existing pigments available [2]. Red-shade pigments produced on an industrial scale usually employ toxic transition metal ions as chromophores like $\mathrm{Al}_{2} \mathrm{O}_{3}: \mathrm{Cr}$ (corundum), $\mathrm{ZnAl}_{2} \mathrm{O}_{4}: \mathrm{Cr}$, $\mathrm{CaSnSiO}_{5}: \mathrm{Cr}$ (sphene), $\mathrm{MnAl}_{2} \mathrm{O}_{4}: \mathrm{Cr}$ (spinel), $\mathrm{Y}_{2} \mathrm{Sn}_{2} \mathrm{O}_{7}: \mathrm{Cr}$ (pyrochlore), and CdS:CdSe (greenockite) [3], where chromium (III) and cadmium (II) ions are an important source of pollution due to their high toxicity. Transitions metal ions are also usually incorporated as chromophores into compounds with stoichiometry $\mathrm{A}_{2} \mathrm{~B}_{2} \mathrm{O}_{7}$ in order to develop coloured compounds. As a result of the high toxicity of several of them $(\mathrm{Cr}, \mathrm{Ni}, \mathrm{Co} \ldots)$, other nontoxic species could play this

\footnotetext{
* Corresponding author.

E-mail address: mir@uji.es (H. Beltrán-Mir).
}

role. Subsequently, lanthanides have the optimum characteristics to replace the toxic chromophore ions in the $\mathrm{A}_{2} \mathrm{~B}_{2} \mathrm{O}_{7}$ structure obtaining environmental friendly pigments $[4,5]$. In the ceramic industry, the most used inorganic red pigment is $\mathrm{Fe}-\mathrm{ZrSiO}_{4}$ (zircon) called pink coral. It is very difficult to control the colour of the $\mathrm{Fe}-\mathrm{ZrSiO}_{4}$ pigments compared to other inorganic pigments, so there must be very precise fine grinding of the starting materials (zirconia and silica). Finer particles can give a better coral pink colour that is more reddish and yellowish in tone. Therefore, this pigment has a great problem of reproducibility and the final colour of the powder can vary widely [6].

Over the last few years, one of the interesting applications of these inorganic pigments is cool roof coatings with high nearinfrared reflectance (NIR) [7-9]. These kind of coatings, for example, can improve internal thermal comfort in buildings and minimize air conditioning. Cool roofing products are made of highly reflective cool materials and can remain cooler than traditional materials when irradiated. White pigments, mainly $\mathrm{TiO}_{2}$ [10], with high solar reflectance are often used as cool materials, but other coloured pigments have been developed because of the necessary aesthetic requirements. Cool pigments are used as coatings on building roofs in order to decrease the temperature in the interior of the house, and consequently, contribute to increased 
indoor thermal comfort levels in hot seasons, resulting in reducing the need for cooling [11]. Many of these coloured pigments with high NIR reflectivity also contain toxic metal elements like $\mathrm{Co}, \mathrm{Pb}$, $\mathrm{Cd}, \mathrm{Cr}$. These are restricted by the current environmental regulations [12-14]. In this way, some new potential cool pigments have been also reported in order to substitute these traditional toxic elements [15-18].

Among all the inorganic structures used for pigments, the pyrochlore structure, $\mathrm{A}_{2} \mathrm{~B}_{2} \mathrm{O}_{7}$, containing trivalent $\mathrm{A}$ and tetravalent $\mathrm{B}$ cations has been studied recently as a new pigment, due to its compositional versatility and high stability $[2,4]$. Of particular interest are oxides with $\mathrm{Zr}$ occupying the B-site and $\mathrm{Y}$ occupying the A-site, $\mathrm{Y}_{2} \mathrm{Zr}_{2} \mathrm{O}_{7}$ (YZ). This compound has been reported to show a moderate anionic conductivity and represent a potential material for use as either electrolytes in solid oxide fuel cells (SOFC) and oxygen separation or in photocatalytic applications [19]. YZ is also a promising host due to the possibility of incorporating a great variety of dopants in the structure. The potential to incorporate different elements provides a greater variety of possibilities highlighting the solid oxide fuel cells $[1,20,21]$ and photoluminescence material applications [22,23].

According to the radius ratios of the ions present [24], $\mathrm{Y}_{2} \mathrm{Zr}_{2} \mathrm{O}_{7}$ acquires a defect fluorite structure under ambient conditions. The ordered pyrochlore structure (space group $\mathrm{Fd}-3 \mathrm{~m}$ ) can be transformed to the disordered defect-fluorite structure (space group Fm$3 \mathrm{~m}$ ) by a random distribution of both cations and anions onto their respective sublattices with the A- and B-site coordination number changing from the 8 and 6 respectively to an average of 7 for both $[25,26]$. Zhang et al. [25] have recently predicted that the coordination of cations in the $\mathrm{Y}_{2} \mathrm{Zr}_{2} \mathrm{O}_{7}$ defect-fluorite varies depending on the species with the average coordination of $\mathrm{Y}$ and $\mathrm{Zr}$ being 7.2 and 6.8 , respectively.

On the other hand, this crystalline structure is significantly flexible, i.e., the lattice can stabilize large amounts of ionic substitutions in each one of its crystalline sites, as well as vacancies and structural defects. This characteristic could be explored to obtain new ceramic pigments using chromophore elements as dopant. As far as we aware, in the field of ceramic pigments, this doped $\mathrm{Y}_{2} \mathrm{Zr}_{2} \mathrm{O}_{7}$ structure has not been reported as yet.

The development of new solids with interesting colour applications is an attractive topic for researchers and industry; especially materials where the substitution of transition ions by lanthanide ions is explored. The use of lanthanides is increasing due to their known low toxicity and their unique optical properties make them a promising material in a wide range of applications, which includes inorganic pigments for ceramic glazes, tunable lasers, or Xray imaging. The terbium ion is one of the lanthanides used as a dopant ion in different structures in the field of new environmental pigments. For example, De la Luz et al. [27] prepared new environmentally inorganic yellow pigments with the general formula $\mathrm{Y}_{1.86-x} \mathrm{M}_{x} \mathrm{~Tb}_{0.14} \mathrm{O}_{3-x / 2}(\mathrm{M}=\mathrm{Ca}$ and/or $\mathrm{Ba})$ by the Pechini method. The intensity of the yellow colour in these samples was related to the presence of $\mathrm{Tb}(\mathrm{IV})$ ions. Athira et al. [3] also studied the role of $\mathrm{Tb}$ ions in the $\mathrm{Y}_{2} \mathrm{Ce}_{2-\mathrm{x}} \mathrm{Tb}_{\mathrm{x}} \mathrm{O}_{7}$ based ceramic pigments resulting in good red hues.

It should also be noted that, iron ion has been used as a chromophore ion in some pigments due to its negligible toxicity, its availability in nature as oxide, and the red shade that it provides to the matrix $[6,28,29]$. Moreover, iron ion is also an environmental friendly chromophore.

Taking into account all of the above-mentioned comments in this work, the synthesis of $\mathrm{Y}_{2-\mathrm{x}} \mathrm{Tb}_{\mathrm{x}} \mathrm{Zr}_{2-\mathrm{y}} \mathrm{Fe}_{\mathrm{y}} \mathrm{O}_{7-\delta}[\mathrm{x}=0,0.25$ and $y=0.15,0.25,0.35]$ by the sol-gel method is reported to obtain new red-shade pigments. Solid solution range and the role of terbium have also been studied. This new pigment has also been prepared to be used in different applications like a ceramic pigment and with a potential pigment for roof insulation. Therefore, the pigment stability in glass has been compared with the stability of the commercial pink coral pigment, and the high NIR reflectance of the same have also been compared with a commercial pigment used in coatings to reflect solar light.

\section{Experimental}

Samples based on $\mathrm{Y}_{2-\mathrm{x}} \mathrm{Tb}_{\mathrm{x}} \mathrm{Zr}_{2-\mathrm{y}} \mathrm{Fe}_{\mathrm{y}} \mathrm{O}_{7-\delta}[\mathrm{x}=0,0.25$ and $\mathrm{y}=0.15$, $0.25,0.35$ ] were prepared by polymeric sol-gel procedure using $\mathrm{Y}\left(\mathrm{OOCCH}_{3}\right)_{3} \cdot \mathrm{H}_{2} \mathrm{O}(99,9 \%$, Strem Chemicals $), \mathrm{Zr}\left(\mathrm{OCH}_{2} \mathrm{CH}_{2} \mathrm{CH}_{3}\right)_{4}(70 \%$ Sigma-Aldrich), $\quad \mathrm{TbCl}_{3} \cdot 6 \mathrm{H}_{2} \mathrm{O} \quad(99,9 \%$ Acros Organics), and $\mathrm{Fe}\left(\mathrm{NO}_{3}\right)_{3} \cdot 9 \mathrm{H}_{2} \mathrm{O}$ (98\% Strem Chemicals) as precursors. All reagents were of an analytical grade and used without further purification. Absolute ethanol (Scharlab, 99.9\%) was used as a solvent with the necessary amount of acetylacetone, acacH (99\% Alfa Aesar) as a stabilizing agent for nonsilicate metal alkoxide precursors. A scheme of the general preparation of the samples is shown in Fig. 1. Zirconium precursor was dissolved in ethanol and acetylacetone (acacH:Zr molar ratio 4:1). Then $\mathrm{Y}\left(\mathrm{OOCCH}_{3}\right)_{3} \cdot \mathrm{H}_{2} \mathrm{O}, \mathrm{Fe}\left(\mathrm{NO}_{3}\right)_{3} \cdot 9 \mathrm{H}_{2} \mathrm{O}$ and $\mathrm{TbCl}_{3} \cdot 6 \mathrm{H}_{2} \mathrm{O}$ (in the samples that contains terbium) were added, and the mixture was stirred for $10 \mathrm{~min}$. This mixture was transferred into a balloon flask and, heated at $70{ }^{\circ} \mathrm{C}$ for $72 \mathrm{~h}$. A gel was formed and it was dried in air at room temperature. Finally, the powder was fired at $1300{ }^{\circ} \mathrm{C}$ for $12 \mathrm{~h}$.

Powders of samples with optimal colour were mixed with one industrial frit (4\% in weight of the pigment) using water as a dispersing medium. Then, the dispersion was applied to white twice-fired bodies, to verify composition stability as a ceramic colourant. A commercial transparent frit was chosen. The frit composition used is given in Table 1. After drying, the pieces were fired in an electric kiln. The heat treatment applied, corresponds to a standard firing cycle used in a ceramic tile industry where the highest temperature of the cycle was $1080{ }^{\circ} \mathrm{C}$ for $5 \mathrm{~min}$. This cycle involves five steps: ramping to $800{ }^{\circ} \mathrm{C}$ in $18 \mathrm{~min}$, heating from $800^{\circ} \mathrm{C}$ to glaze firing temperature in $17 \mathrm{~min}, 5 \mathrm{~min}$ hold at $1080^{\circ} \mathrm{C}$, cooling to $600{ }^{\circ} \mathrm{C}$ in $20 \mathrm{~min}$, and finally cooling to room temperature in $15 \mathrm{~min}$.

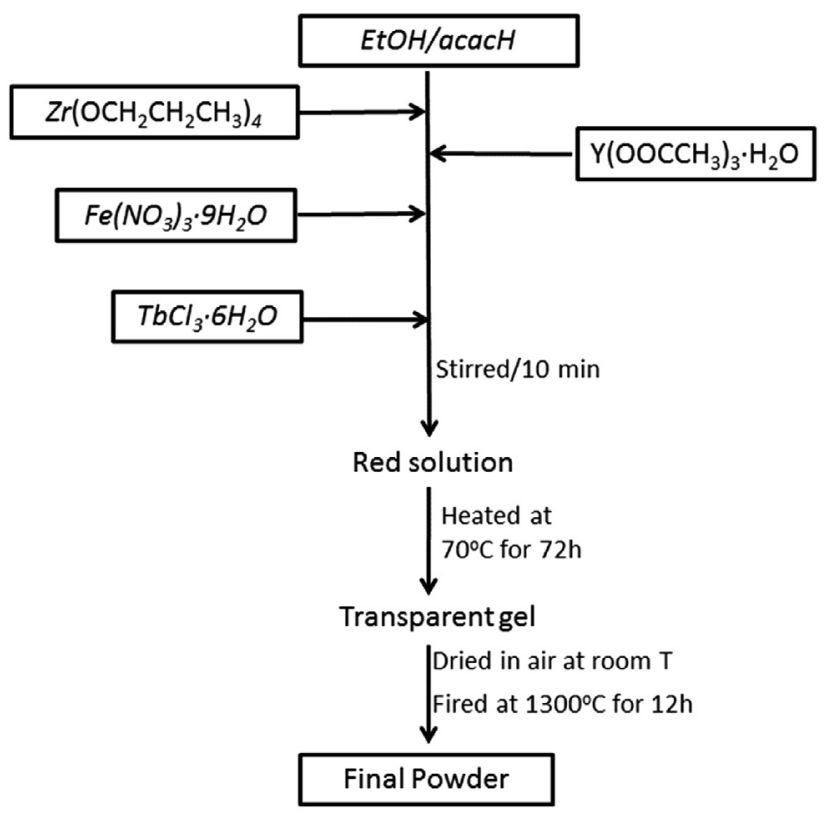

Fig. 1. Scheme for the synthesis procedure. 
Table 1

Frit composition

\begin{tabular}{llllll}
\hline \multicolumn{2}{l}{ Composition (wt\%) } \\
\hline $\mathrm{SiO}_{2}$ & $\mathrm{Al}_{2} \mathrm{O}_{3}$ & $\mathrm{RO}^{\mathrm{b}}$ & $\mathrm{R}_{2} \mathrm{O}^{\mathrm{b}}$ & $\mathrm{ZnO}$ & $\mathrm{ZrO}_{2}$ \\
\hline 67 & 13 & 9.4 & 10 & 0.4 & 0.2 \\
\hline
\end{tabular}

a The percentages do not represent quantitative analyses.

b $\mathrm{R}=$ alkaline or alkaline earth metals.

For the study of samples as cool pigments, the prepared pigments were mixed with siloxane transparent paint (12\% in weight of the pigment) and applied to a ceramic tile $(8 \mathrm{~cm} \times 8 \mathrm{~cm})$. Infrared lamps (Philips, BR 125 IR Red, $250 \mathrm{~W}$ ) were used in order to simulate solar NIR radiation. The indoor temperature difference of the buildings made of foam $(12.5 \mathrm{~cm} \times 8 \mathrm{~cm} \times 8 \mathrm{~cm})$ exposed under the lamps and covered with the ceramic tile treated with the paint (a mixture of the pigment and the siloxane) was measured. A low temperature thermocouple was used to record temperature at 5 min intervals.

\subsection{Characterization}

Phase analysis of the samples was performed by powder XRD using a Bruker D4 Endeavor diffractometer with $\mathrm{CuK}_{\alpha}$ radiation. Data were collected by step-scanning from $2 \theta=20$ to $70^{\circ}$ with a step size of $0.03^{\circ}$ and $6 \mathrm{~s}$ of counting time at each step.

Scanning electron micrographs of the samples were taken on a field emission scanning electron microscope (FE-SEM) JEOL 7001F, equipped with a spectrometer of energy dispersion of X-ray (EDX) from Oxford instruments, using acceleration voltage $=15 \mathrm{kV}$. Semiquantitative analysis was performed from EDX taking an average of ten measurements in different particles. Samples for microstructures and microanalysis determinations were deposited in an aluminium holder and sputtered by platinum.

UV-Visible (UV/Vis) diffuse reflectance spectroscopy, and colourimetric study of the samples were performed on a CARY 500 SCAN VARIAN spectrophotometer in the $380-800 \mathrm{~nm}$ range. $\mathrm{BaSO}_{4}$ was used as a reference. The CIELab colour parameters $L^{*}, a^{*}$, and $b^{*}$ of the samples were determined by coupling analytical software for colour measurements to the Varian spectrophotometer, using a standard illuminant D65, to differentiate the pigment in terms of colour. $L^{*}$ is the lightness axis [black (0) to white (100)], $a^{*}$ is the green $(<0)$ to red $(>0)$ axis, and $b^{*}$ is the blue $(<0)$ to yellow $(>0)$ axis. Near-infrared reflectance of powders and paint were performed on the same spectrophotometer in the $800-2400 \mathrm{~nm}$ range.

Photoluminescence (PL) measurements were performed at room temperature using a CARY ECLIPSE VARIAN fluorescence spectrophotometer in the $450-600 \mathrm{~nm}$ range. Emission spectra were excited at $290 \mathrm{~nm}$.

X-ray photoelectron spectroscopy (XPS) spectra were collected on a SPECS spectrometer equipped with a 150-MCD-9 detector and using a non-monochromatic $\mathrm{Al} \mathrm{K} \alpha(1486.6 \mathrm{eV}) \mathrm{X}$-ray source. Spectra were recorded using an analyser pass energy of $30 \mathrm{eV}$, an Xray power of $50 \mathrm{~W}$ and under an operating pressure of $10^{-9} \mathrm{mbar}$. During data processing of the XPS spectra, binding energy (BE) values were referenced to the $01 \mathrm{~s}$ signal $(529.9 \mathrm{eV})$. Spectra treatment has been performed using the CASA software.

\section{Results and discussion}

Different compositions were synthesized by the sol-gel method. Compositions with general formula $\mathrm{Y}_{2-\mathrm{x}} \mathrm{Tb}_{\mathrm{x}} \mathrm{Zr}_{2-\mathrm{y}} \mathrm{Fe}_{\mathrm{y}} \mathrm{O}_{7-\delta}[\mathrm{x}=0$, 0.25 and $\mathrm{y}=0.15,0.25,0.35$ ] were listed in Table 2 .
Table 2

Compositions prepared based on $\mathrm{Y}_{2-\mathrm{x}} \mathrm{Tb}_{\mathrm{x}} \mathrm{Zr}_{2-\mathrm{y}} \mathrm{Fe}_{\mathrm{y}} \mathrm{O}_{7-\delta}$.

\begin{tabular}{ll}
\hline Ref. & Composition \\
\hline YF015 & $\mathrm{Y}_{2} \mathrm{Zr}_{1.85} \mathrm{Fe}_{0.15} \mathrm{O}_{6.925}$ \\
YF025 & $\mathrm{Y}_{2} \mathrm{Zr}_{1.75} \mathrm{Fe}_{0.25} \mathrm{O}_{6.875}$ \\
YF035 & $\mathrm{Y}_{2} \mathrm{Zr}_{1.65} \mathrm{Fe}_{0.35} \mathrm{O}_{6.825}$ \\
\hline YTF015 & $\mathrm{Y}_{1.75} \mathrm{~Tb}_{0.25} \mathrm{Zr}_{1.85} \mathrm{Fe}_{0.15} \mathrm{O}_{6.925}$ \\
YTF025 & $\mathrm{Y}_{1.75} \mathrm{~Tb}_{0.25} \mathrm{Zr}_{1.75} \mathrm{Fe}_{0.25} \mathrm{O}_{6.875}$ \\
YTF035 & $\mathrm{Y}_{1.75} \mathrm{~Tb}_{0.25} \mathrm{Zr}_{1.65} \mathrm{Fe}_{0.35} \mathrm{O}_{6.825}$ \\
\hline
\end{tabular}

XRD patterns of the samples fired at $1300^{\circ} \mathrm{C}$ are shown in Fig. $2 \mathrm{a}$
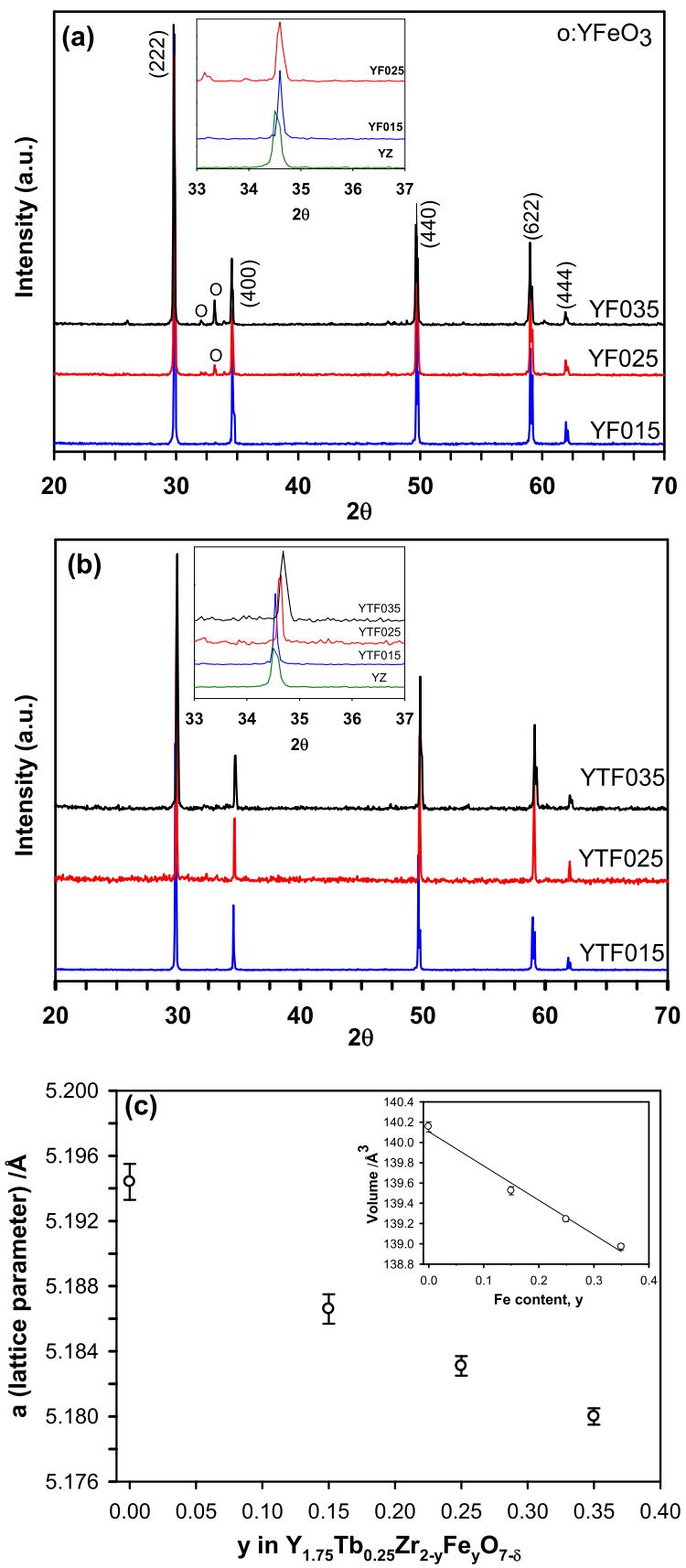

Fig. 2. XRD patterns for powder samples fired at $1300{ }^{\circ} \mathrm{C}$ for $12 \mathrm{~h}$ : (a) YF015, YF025 and YF035; (b) YTF015, YTF025 and YTF035. (c) Lattice parameter, a, and cell volume as a function of $\mathrm{y}$ (iron content) for terbium and iron doped YZ samples. 
and b. The five main characteristics peaks of the defect fluorite structure are identified in both figures. It is established that these peaks, from low to high $2 \theta$ angles, correspond to the (222), (400), (440), (622) and (444) reflections [30]. Therefore, the structure of the main phase in all samples is defect fluorite.

XRD of the samples without terbium are shown in Fig. 2a. YF015 sample was single phase, and a secondary phase was observed when the iron content was higher than 0.15 (YF025 and YF035). In both cases, the secondary phase was attributed to an orthorhombic ferrite phase, $\mathrm{YFeO}_{3}$ [JCPDS-ICDD 86-171]. In contrast, single phase of defect fluorite was observed in all samples that contain terbium, Fig. 2 b.

In order to confirm the formation of the solid solution, the displacement of the Bragg peaks in XRD patterns was analysed and it was compared with the $\mathrm{Y}_{2} \mathrm{Zr}_{2} \mathrm{O}_{7}$ (YZ) peaks. When a solid solution is formed, the position of the diffraction peaks are found to shift slightly with $y$. For a more distinct XDR pattern illustrating this dependence, an example is presented in the insets of Fig. 2a and b, in which (400) diffraction peak for all samples is compared with the $\mathrm{Y}_{2} \mathrm{Zr}_{2} \mathrm{O}_{7}(\mathrm{YZ})$ sample without dopants. In the first case, when iron is the only dopant present, $\mathrm{Y}_{2} \mathrm{Zr}_{2-\mathrm{y}} \mathrm{Fe}_{\mathrm{y}} \mathrm{O}_{7-\delta}$ (inset of Fig. 2a), the diffraction peak for YF015 is shifted with respect to the YZ peak, whereas the peak for YF025 is not shifted with respect to YF015. Therefore, this fact together with the presence of the secondary phase, suggests that the possible limit of iron in this solid solution is 0.15 . In contrast, the (400) peak is shifted towards higher angle sides when the amount of $\mathrm{Fe}$ ion increases for samples with terbium, $\mathrm{Y}_{1.75} \mathrm{~Tb}_{0.25} \mathrm{Zr}_{2-\mathrm{y}} \mathrm{Fe}_{\mathrm{y}} \mathrm{O}_{7-\delta}$ (inset of Fig. 2b). The shifting effect is qualitatively understandable according to Bragg's law $\mathrm{n} \lambda=2 d \sin \theta$, where $d$ is the interplanar distance, $\theta$ is the diffraction angle, and $\lambda$ is the diffraction wavelength. Considering the radii of $\mathrm{Zr}(\mathrm{IV})$ (78 pm, $7 \mathrm{CN}$ ), Y(III) (96 pm, $7 \mathrm{CN}$ ), Fe(III) (72pm, $7 \mathrm{CN}$ ) and $\mathrm{Tb}$ (III) (98 pm, $7 \mathrm{CN}$ ) [31], the above shifting effect of diffraction peaks should be ascribed to the lattice shrinkage caused by the increase of smaller Fe ion concentration. Therefore, a solid solution of iron into the $\mathrm{Y}_{2} \mathrm{Zr}_{2} \mathrm{O}_{7}$ lattice is formed at all studied compositions when the terbium ion is present.

The lattice parameter ( $a$, for cubic phase) and the cell volume of the $\mathrm{Y}_{1.75} \mathrm{~Tb}_{0.25} \mathrm{Zr}_{2-\mathrm{y}} \mathrm{Fe}_{\mathrm{y}} \mathrm{O}_{7-\delta}$ solid solution was also determined and its evolution with the nominal iron concentration is shown in Fig. 2c. The results indicated that there are changes in the lattice parameter, the "a" parameter and therefore, the cell volume, decreases when the concentration of iron increases. This variation in the parameter of network is consistent with the ionic radius values of the cations and dopants involved, and also demonstrate that terbium and iron were incorporated into the $\mathrm{Y}_{2} \mathrm{Zr}_{2} \mathrm{O}_{7}$ structure.

In order to confirm the results obtained by XRD, SEM and EDX analysis were performed in all samples. Grain size in the range $0.5-1.5 \mu \mathrm{m}$ for all samples was observed by SEM. Typical microstructures of the powder samples fired at $1300{ }^{\circ} \mathrm{C}$ for YF025 and YTF035 are shown in Fig. 3. EDX analysis are also shown in Table 3 for these samples. Fig. 3a shows the SEM micrograph of YF025 where two kinds of particles were identified. The major part of the sample is formed by well-sintered particles, but isolated particles through the sample are also identified. EDX results confirmed the presence of a minority secondary phase with $\mathrm{Y}, \mathrm{Fe}$ and $\mathrm{O}$ in this sample without terbium ions, which correspond to the perovskite $\mathrm{YFeO}_{3}$. Similar results were obtained for the YF035 sample, whereas no secondary phases were observed in YF015. SEM and EDX for YTF035, Fig. 3b and Table 3, confirmed that secondary phases were not observed in samples with terbium. The composition is close to the nominal stoichiometry confirming that the desired solid solution was formed in this case. This fact suggests that the presence of terbium promoted the incorporation of the iron ions in the defect fluorite structure and confirmed the results obtained by XRD.
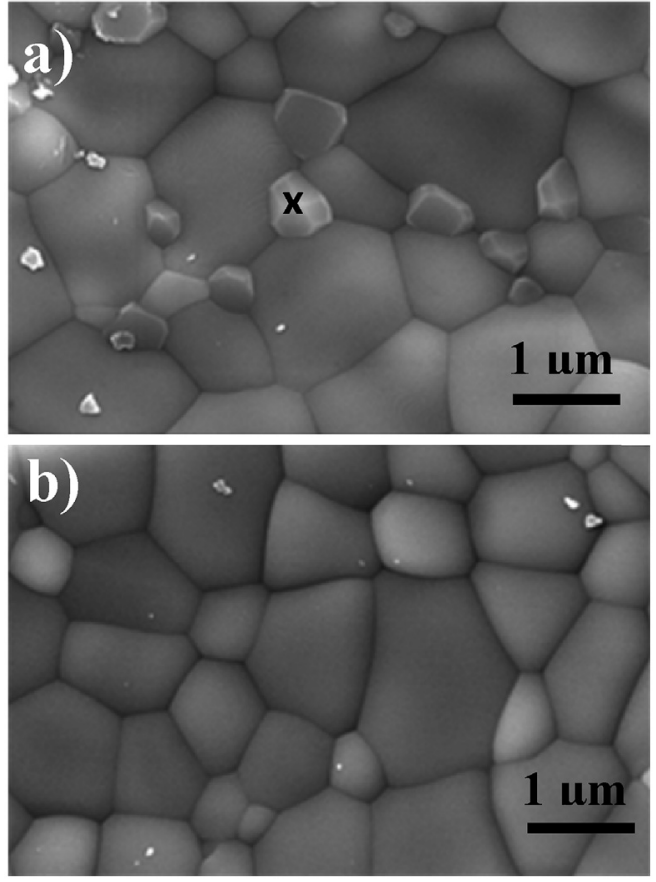

Fig. 3. SEM micrographs of the powders fired at $1300{ }^{\circ} \mathrm{C}$ : a) YF025 b) YTF035. The particle marked with a cross in (a) corresponds to the secondary phase $\mathrm{YFeO}_{3}$ identified by energy dispersive $\mathrm{x}$-ray analysis.

Once the structural evolution and homogeneity with composition of the samples was established, optical properties were investigated by UV/Vis spectroscopy. Optical spectra of the powders, which present single defect fluorite phase, are shown in Fig. 4 in the range $380-760 \mathrm{~nm}$. All powders exhibited a good red-shade colour after fired at $1300^{\circ} \mathrm{C}$ for $12 \mathrm{~h}$, but the intensity of the colour was different. The presence of terbium in the samples plays an important role in the UV/Vis absorption. A broad absorption band in the blue region of the spectra was observed in all samples with terbium. The presence of a broad absorption band around $400 \mathrm{~nm}$ is related to the presence of $\mathrm{Tb}(\mathrm{IV})$ ions, since this band is ascribed to a $\mathrm{Tb}^{4+}-\mathrm{O}^{2-}$ charge-transfer $[32,33]$. The intensity of the absorption in the blue region of the optical spectra increases when $\mathrm{Tb}$ is present and suggests a greater red colouration.

In order to study in more detail the effect of terbium ions in the structure, photoluminescence studies were performed on samples that contain terbium. PL measurements were conducted in order to evaluate whether $\mathrm{Tb}(\mathrm{IV})$ ions could be presented in the samples studied (YTF015, YTF025 and YTF035). The oxidation state of terbium ions, $\mathrm{Tb}(\mathrm{III})$ or $\mathrm{Tb}(\mathrm{IV})$, plays an important role for their fluorescence intensity. $\mathrm{Tb}(\mathrm{IV})$ ions do not participate in the emission process and they act as a fluorescence quenching centre, reducing the fluorescence efficiency [27]. Fig. 5 shows the emission spectra at room temperature for YTF015, YTF025 and YTF035 sintered in air at $1300{ }^{\circ} \mathrm{C}$ for $12 \mathrm{~h}$. These emission spectra were registered using the same wavelength excitation $(280 \mathrm{~nm})$ in the range of $450-600 \mathrm{~nm}$, where the characteristics ${ }^{5} \mathrm{D}_{4} \rightarrow{ }^{7} \mathrm{~F}_{\mathrm{J}}$ transitions of $\mathrm{Tb}$ (III) appear. The emission spectra for all samples consist of three main lines at $488 \mathrm{~nm}\left({ }^{5} \mathrm{D}_{4} \rightarrow{ }^{7} \mathrm{~F}_{6}\right), 543 \mathrm{~nm}\left({ }^{5} \mathrm{D}_{4} \rightarrow{ }^{7} \mathrm{~F}_{5}\right)$ and $586 \mathrm{~nm}\left({ }^{5} \mathrm{D}_{4} \rightarrow{ }^{7} \mathrm{~F}_{4}\right)$ which correspond to the emission bands of $\mathrm{Tb}$ (III) [34,35]. However, the intensity of these bands was different depending on the sample composition, showing that when the iron content increases the intensity of these bands decreases. In particular, the intensity of the bands in YTF035 sample is much lower than YTF015. It is related to the different amount of $\mathrm{Tb}(\mathrm{III})$ 
Table 3

EDX analysis for YF025 and YTF035 samples in mole \% of each element.

\begin{tabular}{|c|c|c|c|c|}
\hline \multirow[t]{2}{*}{ Element } & \multicolumn{2}{|l|}{ YF025 } & \multicolumn{2}{|l|}{ YTF035 } \\
\hline & Majority phase ${ }^{a}$ & Minority phase (x) & Majority phase ${ }^{a}$ & Minority phase \\
\hline $\mathrm{Y}$ & $17.7 \pm 0.3$ & 18.41 & $16.10 \pm 0.4$ & - \\
\hline $\mathrm{Zr}$ & $16.6 \pm 0.2$ & - & $16.14 \pm 0.5$ & - \\
\hline $\mathrm{Fe}$ & $1.73 \pm 0.4$ & 15.59 & $3.06 \pm 0.2$ & - \\
\hline $\mathrm{Tb}$ & - & - & $2.06 \pm 0.3$ & - \\
\hline $\mathrm{O}$ & $63.97 \pm 0.1$ & 66 & $62.64 \pm 0.2$ & - \\
\hline
\end{tabular}

${ }^{\text {a }}$ Average content of the elements from the EDX analysis in different particles.

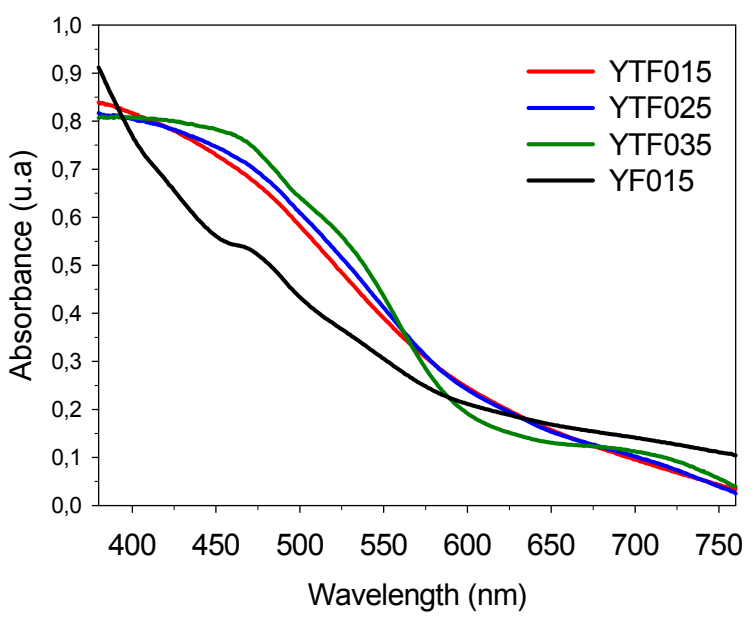

Fig. 4. Optical spectra for samples fired at $1300^{\circ} \mathrm{C}$ for $12 \mathrm{~h}$.

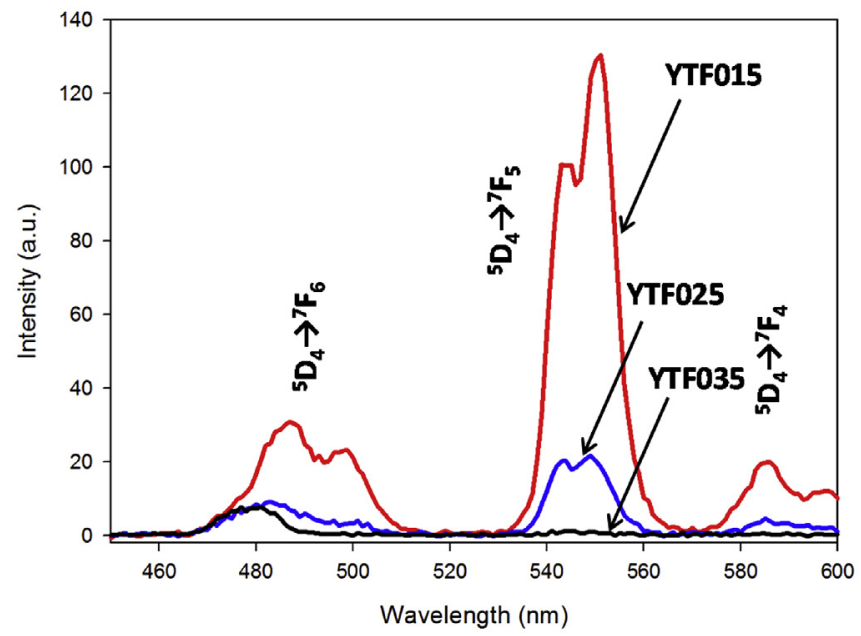

Fig. 5. Photoluminescence spectra for YTF015, YTF025 and YTF035 powders after fired at $1300{ }^{\circ} \mathrm{C}$ for $12 \mathrm{~h}$.

ions in the samples, which decreases when the iron content increases, and therefore, the amount of Tb(IV) ions increases.

With a view to verifying the oxidation state of the iron ions in two selected samples (YF015 and YTF015), XPS analysis was performed for one composition of iron, $\mathrm{y}=0.15$, with and without terbium. The background due to inelastic scattered electrons was calculated for each reference spectrum, using the formalism of Tougaard [36]. The oxygen $1 \mathrm{~s}$ was used as an internal standard to correct for charging of the catalyst samples. XPS peaks of Fe $2 \mathrm{p}_{3 / 2}$ and Fe2 $\mathrm{p}_{1 / 2}$ for the YF015 and YTF015 samples are shown in Fig. 6. The binding energy difference between the $\mathrm{Fe} 2 \mathrm{p}_{3 / 2}$ peak and the
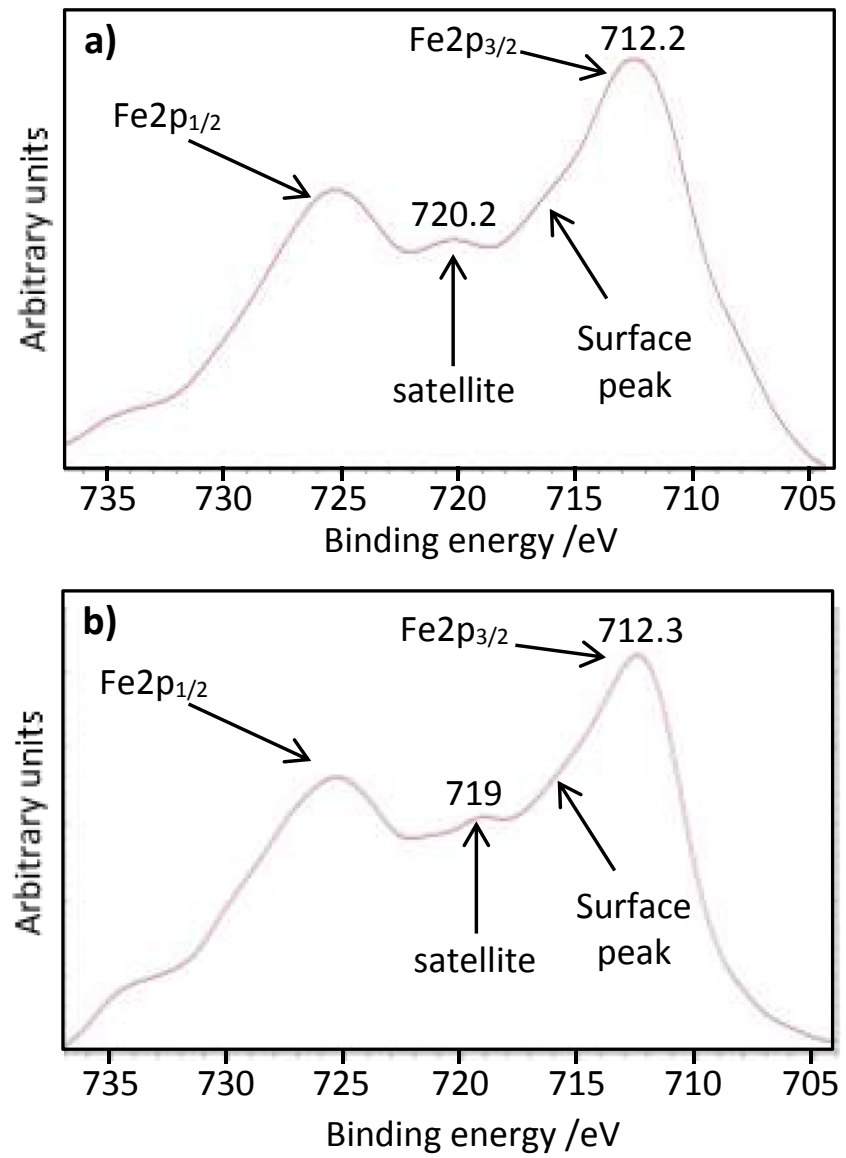

Fig. 6. XPS spectrum of Fe2p for samples: (a) YF015 and (b) YTF015.

satellite peak is approximately $8 \mathrm{eV}$ in YF015. This binding energy difference between these two peaks is consistent with the values observed in the literature for $\mathrm{Fe}_{2} \mathrm{O}_{3}$, and it is a clear evidence of the existence of $\mathrm{Fe}(\mathrm{III})$ ions in this sample [37-39]. However, the difference of the shoulder satellite peak and the blinding energy in the YTF015 sample is around $6.5 \mathrm{eV}$. This result is consistent with the existence of the $\mathrm{Fe}(\mathrm{II})$ in the sample [37-39]. However, the presence of the surface peak indicated that $\mathrm{Fe}(\mathrm{III})$ exists in both samples. According to Grosvenor et al. [38], a possible cause of the presence of this surface peak is the reduction in the coordination of the molecules located at the surface after the surface has been cleaved in a vacuum or crushed under either an $\mathrm{N}_{2}$ or an $\mathrm{Ar}$ atmosphere. According to this result, it is possible to conclude that the amount of $\mathrm{Fe}(\mathrm{II})$ ions in sample with terbium is higher than sample without this ion.

Once the oxidation states of terbium and iron ions were identified, in order to understand the variation of the colour, it is 
possible to establish the following discussion:

According to XPS, $\mathrm{Fe}(\mathrm{II})$ is present in the $\mathrm{Y}_{1.75} \mathrm{~Tb}_{0.25} \mathrm{Zr}_{1.85} \mathrm{Fe}_{0.15} \mathrm{O}_{6.925}$ (YTF015) sample. The presence of $\mathrm{Fe}(\mathrm{II})$ ions like the mainly oxidation state for iron in this sample could be related to the presence of $\mathrm{Tb}(\mathrm{IV})$. This oxidation process of $\mathrm{Tb}(\mathrm{III})$ to $\mathrm{Tb}(\mathrm{IV})$ involves the reduction of part of the $\mathrm{Fe}(\mathrm{III})$ to $\mathrm{Fe}(\mathrm{II})$. The presence of $\mathrm{Tb}(\mathrm{IV})$ has been previously observed by PL measurements in other systems [27], where the presence of divalent cations like calcium can promote the oxidation process of $\mathrm{Tb}(\mathrm{III})$ to $\mathrm{Tb}(\mathrm{IV}$ ) ions in samples of $\mathrm{Tb}$ (III) doped $\mathrm{Y}_{2} \mathrm{O}_{3}$.

The amount of terbium is the same in all samples, however, the mainly oxidation state of $\mathrm{Tb}$ ions is changing when the amount of iron increases, as can be observed by PL measurements. The amount of $\mathrm{Tb}(\mathrm{IV})$ ions increase when the amount of iron in the sample also increase. Nevertheless, due to the high presence of iron with respect to $\mathrm{Tb}$ in YTF035, only a certain amount of $\mathrm{Fe}(\mathrm{III})$ was reduced to $\mathrm{Fe}(\mathrm{II})$, and the rest remained as $\mathrm{Fe}(\mathrm{III})$ ions. As a consequence of this trend, the amount of Fe(III) in YTF035 was higher than YTF015. If the colouration mechanism depends on the $\mathrm{Fe}$ (III) concentration, it could be related to the colour of the pigment, and especially, with the intensity of the red colour obtained in each case.

The study of the colour was only based on the samples that presented single phase by XRD. Table 4 shows the chromatic coordinates of the powder samples together with the chromatic coordinates of the commercial pigment. All samples present a good red-shade colouration in the powder form, highlighting the YTF035 sample with the higher value of $a^{*}\left(a^{*}>0\right.$ red colouration and $b^{*}>0$ yellow colouration). Moreover, the $a^{*}$ and $L^{*}$ values were similar to those obtained for the commercial pigment. According to the values obtained for YF015 and YFT015 samples, terbium has a clear effect on the colouration, increasing the $\mathrm{a}^{*}$ coordinate and decreasing the lightness ( $\mathrm{L}^{*}$ coordinate). On the other hand, an increase of the $a^{*}$ and $b^{*}$ coordinate was also observed with the increasing of the amount of iron in the structure for samples containing terbium. This evolution is related to the oxidation states of Fe and Tb ions. YTF035 was the sample that presented the better chromatic coordinates and, based on XPS and PL results, $\mathrm{Tb}(\mathrm{IV})$ and $\mathrm{Fe}(\mathrm{III})$ were the mainly oxidation state together with $\mathrm{Fe}(\mathrm{II})$ in this sample.

From these results, it is possible to conclude that: first, chromatic coordinates were consistent with XRD, and demonstrated that solid solution was formed; second, it was also consistent with the UV/Vis results where there exists a greater absorption in the blue region of the visible spectra where the samples contain terbium; third, the pigment presented the best chromatic coordinates when the amount of $\mathrm{Fe}(\mathrm{III})$, and also $\mathrm{Tb}(\mathrm{IV})$ ions, was higher.

\subsection{Evaluation of the colouring performance of the pigment}

In order to determine the stability of the best red-shade powders (YTF025 and YFT035) prepared at $1300{ }^{\circ} \mathrm{C}$ after glazing, a powder/frit mixture was prepared and fired according the cycle set

\section{Table 4}

Chromatic coordinates $\left(\mathrm{L}^{*}, \mathrm{a}^{*}\right.$ and $\left.\mathrm{b}^{*}\right)$ for powder samples fired at $1300{ }^{\circ} \mathrm{C}$ for $12 \mathrm{~h}$. The $\mathrm{L}^{*} \mathrm{a}^{*} \mathrm{~b}^{*}$ values for the commercial pigment (pink coral) are also included.

\begin{tabular}{llrl}
\hline Ref. & L $^{*}$ & $\mathrm{a}^{*}$ & $\mathrm{~b}^{*}$ \\
\hline YF015 & 84 & 5 & 25 \\
YTF015 & 65 & 12 & 25 \\
YTF025 & 70 & 17 & 34 \\
YTF035 & 70 & 23 & 38 \\
Commercial & 71 & 25 & 18 \\
\hline
\end{tabular}

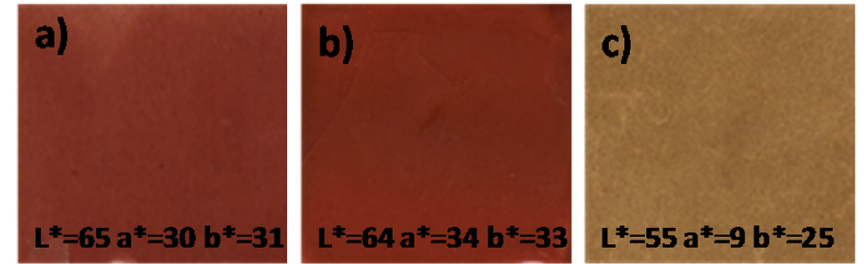

Fig. 7. Photographs and chromatic coordinates of the ceramic tiles for (a) commercial pigment and (b) YTF035 after mixed with the frit; (c) YTF025 after mixed with the paint.

out in the experimental part at the maximum temperature of $1080^{\circ} \mathrm{C}$. The pigment colour was also red after glazing with the frit for the two samples, but YTF035 showed the best chromatic coordinates. In this sample, red $a^{*}$ coordinate $\left(a^{*}=34\right)$ was better than the value obtained for the industrial pink coral red pigment $\left(a^{*}=30\right)$ mixed with the same frit. Moreover, $b^{*}$ values for this composition $\left(b^{*}=32\right)$ were very close to that of the industrial pigment $\left(b^{*}=31\right)$. Therefore, good chemical and thermal stability into the frit was obtained and good red colouration was observed for the $\mathrm{Y}_{1.75} \mathrm{~Tb}_{0.25} \mathrm{Fe}_{0.35} \mathrm{Zr}_{1.65} \mathrm{O}_{6.825}$ composition. Photographs for this composition and the commercial pigment, after mixed with the frit, are shown in Fig. 7a and b, respectively. Chromatic coordinates of the final colour are also indicated in this figure. These results make this pigment a potential candidate for environmental red ceramic pigment.

\subsection{Evaluation of the thermal performance of the pigmented coatings}

Powder compositions having the best chromatic properties (YTF025 and YTF035) were evaluated for their thermal performance by incorporating them into a coating paint.

First, in order to test the potential applications of this pigment like an IR reflective pigment, spectral reflectance in the NIR region for the YTF025 and YTF035 powder samples were done. YTF025 powder presented a high NIR reflectance reaching values of around $90 \%$, Fig. 8 . This value is higher than values reached by other similar "cool pigments" in the literature $[11,18,40]$. In order to study the coating applications of this novel red pigment, YTF025 was mixed with siloxane transparent paint. Siloxane is an impermeable paint providing a protection from the rain water and ice increasing the

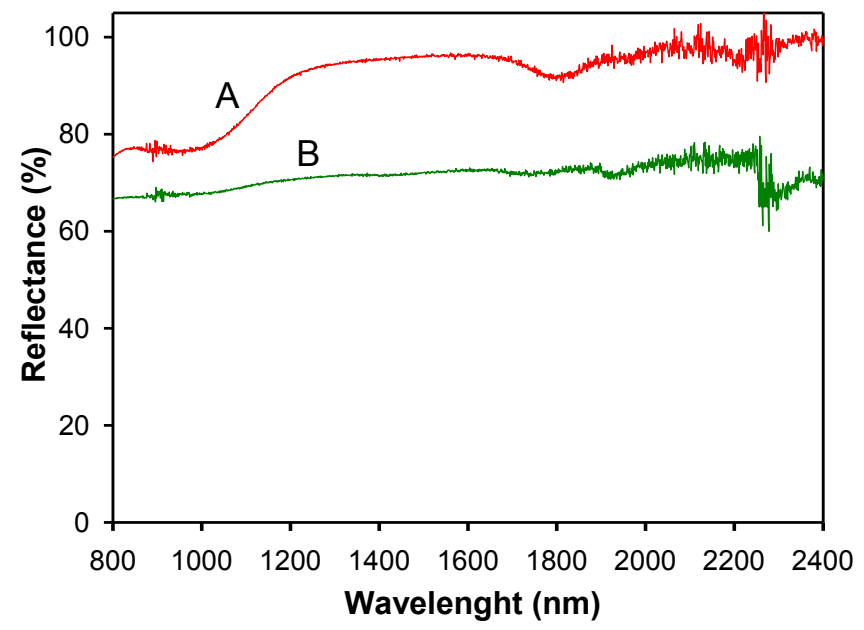

Fig. 8. NIR reflectance spectra of YTF025 powder (A) and the mixture of paint and YTF025 powder (B). 
Table 5

Indoor temperature at variable times for YTF025 pigment mixed with a siloxane paint used as cool roofing material.

\begin{tabular}{|c|c|c|c|c|c|c|}
\hline Time (min) & & 10 & 15 & 20 & 25 & 30 \\
\hline \multirow[t]{3}{*}{ Temperature } & $\begin{array}{l}\text { Paint (T1) } \\
\text { [0\% YTF025] }\end{array}$ & 28.5 & 29.7 & 30.4 & 31.8 & 32.8 \\
\hline & $\begin{array}{l}\text { Paint (T2) } \\
\text { [12\% YTF025] }\end{array}$ & 27 & 29 & 29.8 & 30.7 & 31.5 \\
\hline & $\Delta \mathrm{T}(\mathrm{T} 1-\mathrm{T} 2)$ & 1.5 & 0.7 & 0.6 & 1.1 & 1.3 \\
\hline
\end{tabular}

durability of the surface where the paint is applied. Therefore, it seems very interesting to add to these features a high reflectance in order to obtain paint for exterior coatings. Fig. 8 also shows the NIR reflectance of YTF025 mixed with the paint, reaching NIR reflectance values of around $70 \%$.

With the aim to test the potential application of this pigment as a cool roofing material, an evaluation of the different indoor air temperature was carried out after a foam building was covered with a painted ceramic tile. Fig. 7c shows that the YTF025 powder showed a good stability in the siloxane paint after applying it on the surface of the ceramic tile, decreasing slightly the initial colour of the powder. Table 5 lists the air temperature of different coatings (with and without pigment) at several times. The indoor air temperature for the paint without pigment (T1) is higher than the mixture of paint and pigment (T2). About 10 min later, the air temperature difference $(\Delta \mathrm{T})$ was about $1.5^{\circ} \mathrm{C}$. The $\Delta \mathrm{T}$ reaches $1.3^{\circ} \mathrm{C}$ after $30 \mathrm{~min}$. Therefore, the pigmented coatings could be used as cool roofing materials in future.

The optimal NIR reflectance and the potential applications in paint, together with the use of environmental friendly elements and the good red colouration; make this pigment optimal for these kind of applications.

\section{Conclusions}

A new, red-shade, environmentally friendly pigment based on $\mathrm{Y}_{2-\mathrm{x}} \mathrm{Tb}_{\mathrm{x}} \mathrm{Zr}_{2-\mathrm{y}} \mathrm{Fe}_{\mathrm{y}} \mathrm{O}_{7-\mathrm{y} / 2}$ was prepared by a polymeric sol-gel method and after calcination at $1300{ }^{\circ} \mathrm{C}$. Single phase of defect fluorite structure was obtained by XRD when terbium ion is presented in the structure. SEM/EDX confirmed the results obtained by XRD, and showed that homogenous samples with a particle size of around $0.5-1.5 \mu \mathrm{m}$ were synthesized. Optical properties were investigated by UV/Vis absorption measurements, and the colour of the pigment was determined by CIELab chromatic coordinates. The presence of terbium provided a higher intensity of the absorption in the blue region of the UV/Vis spectra obtaining a greater red colouration. Moreover, when the amount of iron increases this increase also occurs, leading to a better red colouration of the pigment.

Oxidation states of terbium and iron ions were related to the colour of the final powders. The oxidation state of both was studied by XPS, photoluminescence and it was related to the final colouration of the samples. Pigments presented best chromatic coordinates when the amount of $\mathrm{Fe}(\mathrm{III})$ ions, and also $\mathrm{Tb}(\mathrm{IV})$ ions, was higher (YTF035).

Powder samples of the pigment with the best red colouration (YTF035) have a good chemical and thermal stabilization into the frit, presenting slightly better chromatic coordinates than those of the commercial ceramic pigment currently used. Red-shade colour was kept after the application on glazes.

This pigment presented an optimal NIR reflectance in a powder form, and in a mixture with a siloxane paint applied in a ceramic tile. The optimal NIR reflectance and the potential applications in paint, together with the use of environmental friendly elements and the good red colouration; make this pigment optimal for applications like "cool pigments" used for building roofing materials.
Therefore, this new pigment could be a multifunctional material for applications such as ceramic tiles or roofing materials.

\section{Acknowledgements}

We thank the "Universitat Jaume I"-project No. P11B2013-65 (MJ, AS, HB, EC) for financial support. M.J. also thanks this project for a fellowship.

\section{References}

[1] Kumar M, Raj IA, Pattabiraman R. $\mathrm{Y}_{2} \mathrm{Zr}_{2} \mathrm{O}_{7}$ (YZ)-pyrochlore based oxide as an electrolyte material for intermediate temperature solid oxide fuel cells (ITSOFCs)-Influence of Mn addition on YZ. Mater Chem Phys 2008;108:102-8.

[2] Pavlov RS, Castello JBC. New red-shade ceramic pigments based on $\mathrm{Y}_{2} \mathrm{Sn}_{2-\mathrm{x}} \mathrm{Cr}$ ${ }_{x} \mathrm{O}_{7-\delta}$ pyrochlore solid solutions. J Am Ceram Soc 2002;202:1197-202.

[3] Raj AKV, Prabhakar Rao P, Sameera S, Divya S. Pigments based on terbiumdoped yttrium cerate with high NIR reflectance for cool roof and surface coating applications. Dye Pigment 2015;122:116-25.

[4] Stránská L, Šulcová P, Vlček M. Synthesis and properties of inorganic pigments based on pyrochlore compounds with different lanthanides. J Therm Anal Calorim 2013;113:127-35.

[5] Martos M, Julian-López B, Cordoncillo E, Escribano P. Structural and spectroscopic study of a novel erbium titanate pink pigment prepared by sol-gel methodology. J Phys Chem B 2008;112:2319-25.

[6] Pyon K, Lee B. Effect of iron content and annealing temperature on the color characteristics of $\mathrm{Fe}_{\mathrm{ZrSiO}}$ coral pink pigments synthesized by sol-gel method. J Ceram Soc Jpn 2009;117:258-63.

[7] Taylor DJ, Fleig PF, Page RA. Characterization of nickel titanate synthesized by sol - gel processing. Thin Solid Films 2002;408:104-10.

[8] Zou J, Zhang P, Liu C, Peng Y. Highly dispersed (Cr, Sb)-co-doped rutile pigments of cool color with high near-infrared reflectance. Dye Pigment 2014;109:113-9.

[9] Levinson R, Berdahl P, Akbari H. Solar spectral optical properties of pigmentsPart II: survey of common colorants. Sol Energy Mater Sol Cells 2005;89: $351-89$.

[10] Fang V, Kenedy J, Futter J, Manning J. A review of near infrared reflectance properties of metal oxide nanostructures. GNS Sci Rep 2013;39:23.

[11] Radhika S, Sreeram KJ, Nair BU. Effective synthesis route for red-brown pigments based on Ce-Pr-Fe-O and their potential application for near infrared reflective surface coating. J Chem Sci 2014;126:65-73.

[12] Tao Z, Zhang W, Huang Y, Wei D, Seo HJ. A novel pyrophosphate $\mathrm{BaCr}_{2}\left(\mathrm{P}_{2} \mathrm{O}_{7}\right)_{2}$ as green pigment with high NIR solar reflectance and durable chemical stability. Solid State Sci 2014;34:78-84.

[13] Levinson R, Berdahl P, Akbari H, Miller W, Joedicke I, Reilly J, et al. Methods of creating solar-reflective nonwhite surfaces and their application to residential roofing materials. Sol Energy Mater Sol Cells 2007;91:304-14.

[14] Libbra A, Tarozzi L, Muscio A, Corticelli MA. Spectral response data for development of cool coloured tile coverings. Opt Laser Technol 2011;43: 394-400.

[15] Han A, Zhao M, Ye M, Liao J, Zhang Z, Li N. Crystal structure and optical properties of $\mathrm{YMnO}_{3}$ compound with high near-infrared reflectance. Sol Energy 2013;91:32-6.

[16] Hao T, Yang C, Rao X, Wang J, Niu C, Su X. Facile additive-free synthesis of iron oxide nanoparticles for efficient adsorptive removal of Congo red and $\mathrm{Cr}(\mathrm{VI})$. Appl Surf Sci 2014;292:174-80.

[17] Kumari LS, George G, Rao PP, Reddy MLP. The synthesis and characterization of environmentally benign praseodymium-doped $\mathrm{TiCeO}_{4}$ pigments. Dye Pigment 2008:77:427-31.

[18] Zhao M, Han A, Ye M, Wu T. Preparation and characterization of $\mathrm{Fe}^{3+}$ doped $\mathrm{Y}_{2} \mathrm{Ce}_{2} \mathrm{O}_{7}$ pigments with high near-infrared reflectance. Sol Energy 2013;97: $350-5$.

[19] Tong Y, Xue P, Jian F, Lu L, Wang X, Yang X. Preparation and characterization of $\mathrm{Y}_{2} \mathrm{Zr}_{2} \mathrm{O}_{7}$ nanocrystals and their photocatalytic properties. Mater Sci Eng B 2008; 150:194-8.

[20] Kumar M, Kulandainathan MA, Raj IA, Chandrasekaran R, Pattabiraman R Electrical and sintering behaviour of $\mathrm{Y}_{2} \mathrm{Zr}_{2} \mathrm{O}_{7}(\mathrm{YZ})$ pyrochlore based materials: the influence of bismuth. Mater Chem Phys 2005;92:303-9.

[21] Yang F, Wang Y, Zhao X, Xiao P. Enhanced ionic conductivity in pyrochlore 
and fluorite mixed phase yttrium-doped lanthanum zirconate. J Power Sources 2015;273:290-7.

[22] Zhang A, Lü M, Qiu Z, Zhou Y, Ma Q. Multiband luminescence of Eu ${ }^{3+}$ based on $\mathrm{Y}_{2} \mathrm{Zr}_{2} \mathrm{O}_{7}$ nanocrystals. Mater Chem Phys 2008;109:105-8.

[23] Du Q, Zhou G, Zhou J, Jia X, Zhou H. Enhanced luminescence of novel $\mathrm{Y}_{2} \mathrm{Zr}_{2} \mathrm{O}_{7}$ : $\mathrm{Dy}^{3+}$ phosphors by $\mathrm{Li}^{+}$co-doping. J Alloys Compd 2013;552:152-6.

[24] Subramanian MA, Aravamudan G, Subba Rao GV. Oxide pyrochlores - a review. Prog Solid State Chem 1983;15:55-143.

[25] Zhang Z, Middleburgh SC, Reyes MDL, Lumpkin GR, Kennedy BJ, Blanchard PER, et al. Gradual structural evolution from pyrochlore to defectfluorite in $\mathrm{Y}_{2} \mathrm{Sn}_{2-\mathrm{x}} \mathrm{Zr}_{\mathrm{x}} \mathrm{O}_{7}$. J Phys Chem 2013;117:26740-9.

[26] Norberg ST, Hull S, Eriksson SG, Ahmed I, Kinyanjui F, Biendicho JJ. Pyrochlore to fluorite transition: the $\mathrm{Y}_{2}\left(\mathrm{Ti}_{1-\mathrm{x}} \mathrm{Zr}_{\mathrm{x}}\right)_{2} \mathrm{O}_{7}(0.0<\mathrm{x}<1.0)$ system. Chem Mater 2012;24:4294-300.

[27] De la Luz V, Prades M, Beltrán H, Cordoncillo E. Environmental-friendly yellow pigment based on $\mathrm{Tb}$ and $\mathrm{M}\left(\mathrm{M}=\mathrm{Ca}\right.$ or $\mathrm{Ba}$ ) co-doped $\mathrm{Y}_{2} \mathrm{O}_{3}$. J Eur Ceram Soc 2013;33:3359-68.

[28] Cappelletti G, Ardizzone S, Fermo P, Gilardoni S. The influence of iron content on the promotion of the zircon structure and the optical properties of pink coral pigments. J Eur Ceram Soc 2005;25:911-7.

[29] Longo E, Santos-Ju MRMC, Sczancoski JC, Santos V, Cavalcante LS, Nunes MGB. Sol-gel synthesis and characterization of $\mathrm{Fe}_{2} \mathrm{O}_{3} \cdot \mathrm{CeO}_{2}$ doped with Pr ceramic pigments. J Sol-Gel Sci Technol 2008:47:38-43.

[30] Zou X, Zhou G, Yi H, Zhang G, Wang S. Fabrication of transparent $\mathrm{Y}_{2} \mathrm{Zr}_{2} \mathrm{O}_{7}$ ceramics from combustion-synthesized powders. J Am Ceram Soc 2011;94:
$1002-4$.

[31] Shannon RD. Revised effective ionic radii and systematic studies of interatomic distances in halides and chalcogenides. Acta Crystallogr Sect A 1976;32:751-67.

[32] Lammers MJJ, Donker H, Blasse G. The luminescence of $\mathrm{Eu}^{3+}, \mathrm{Tb}^{3+}$ and $\mathrm{Tm}^{3-}$ activated $\mathrm{Gd}_{2} \mathrm{BaZnO}_{5}$ and $\mathrm{La}_{2} \mathrm{BaZnO}_{5}$. Mater Chem Phys 1985;13:527-39.

[33] Dachraoui H, Rupp RA, Lengyel K, Ellabban MA, Fally M, Corradi G, et al Photochromism of doped terbium gallium garnet. Phys Rev B 2006;74:1-11.

[34] Wakefield G, Keron HA, Dobson PJ, Hutchison JL. Structural and optical properties of terbium oxide nanoparticles 1999;60:503-8.

[35] Gao L, An Y, Zhu H, Wang L, Chen J, Wang N, et al. Hydrothermal synthesis and photoluminescence properties of $\mathrm{Y}_{2} \mathrm{Zr}_{2} \mathrm{O}_{7}: \mathrm{Tb}^{3+}$ phosphors. I Mater $\mathrm{SC}$ 2010;46:1337-40.

[36] Tougaard S. Low energy inelastic electron scattering properties of noble and transitions metals. Solid State Commun 1987;61:547-9.

[37] Grosvenor AP, Kobe BA, Biesinger MC, Mcintyre NS. Investigation of multiplet splitting of Fe2p XPS spectra and bonding in iron compounds 2004;36: $1564-74$.

[38] Roosendaal SJ, Van Asselen B, Elsenaar JW, Vredenberg AM, Habraken FHPM. The oxidation state of $\mathrm{Fe}(100)$ after initial oxidation in $\mathrm{O}_{2}$. Surf Sci 1999;442: $329-37$.

[39] Yamashita T, Hayes P. Analysis of XPS spectra of $\mathrm{Fe}^{2+}$ and $\mathrm{Fe}^{3+}$ ions in oxide materials. Appl Surf Sci 2008;254:2441-9.

[40] Bendiganavale AK, Malshe VC. Infrared reflective inorganic pigments. Recent Pat Chem Eng 2008;1:67-79. 


\subsection{Discusión de los resultados}

En el presente estudio se han conseguido sintetizar disoluciones sólidas con estequiometria $\mathrm{Y}_{2-\mathrm{x}} \mathrm{Tb}_{\mathrm{x}} \mathrm{Zr}_{2-\mathrm{y}} \mathrm{Fe}_{\mathrm{y}} \mathrm{O}_{7-\delta}[\mathrm{x}=0,0.25$ e $\mathrm{y}=0.15,0.25,0.35]$ a través del método de síntesis sol-gel polimérico calcinadas a $1300{ }^{\circ} \mathrm{C}$ durante $12 \mathrm{~h}$. Las composiciones y las correspondientes referencias de las muestras sintetizadas se muestran en la tabla 3.

Tabla 3. Composiciones preparadas basadas en $\mathrm{Y}_{2-\mathrm{x}} \mathrm{Tb}_{\mathrm{x}} \mathrm{Zr}_{2-\mathrm{y}} \mathrm{Fe}_{\mathrm{y}} \mathrm{O}_{7-\delta}$.

\begin{tabular}{c|c} 
Referencia & \multicolumn{1}{c}{ Composición } \\
\hline \hline YF015 & $\mathrm{Y}_{2} \mathrm{Zr}_{1.85} \mathrm{Fe}_{0.15} \mathrm{O}_{6.925}$ \\
YF025 & $\mathrm{Y}_{2} \mathrm{Zr}_{1.75} \mathrm{Fe}_{0.25} \mathrm{O}_{6.875}$ \\
YF035 & $\mathrm{Y}_{2} \mathrm{Zr}_{1.65} \mathrm{Fe}_{0.35} \mathrm{O}_{6.825}$ \\
\hline YTF015 & $\mathrm{Y}_{1.75} \mathrm{~Tb}_{0.25} \mathrm{Zr}_{1.85} \mathrm{Fe}_{0.15} \mathrm{O}_{6.925}$ \\
YTF025 & $\mathrm{Y}_{1.75} \mathrm{~Tb}_{0.25} \mathrm{Zr}_{1.75} \mathrm{Fe}_{0.25} \mathrm{O}_{6.875}$ \\
YTF035 & $\mathrm{Y}_{1.75} \mathrm{~Tb}_{0.25} \mathrm{Zr}_{1.65} \mathrm{Fe}_{0.35} \mathrm{O}_{6.825}$
\end{tabular}

Se obtuvieron disoluciones sólidas con una estructura de fluorita defectuosa en todas las muestras que contenían terbio y hierro (YTF015, YTF025, YTF035), así como en la muestra sin terbio con menor cantidad de hierro (YF015). Para ello se caracterizaron las muestras estructuralmente por difracción de rayos X (DRX) y microestructuralmente por microscopía electrónica de barrido (SEM) y microanálisis por energías dispersivas de rayos $\mathrm{X}$ (EDX). Las muestras sin terbio con cantidades de hierro superiores a 0.15 es decir, YF025 y YF035, presentaron una fase secundaria de perovsquita $\left(\mathrm{YFeO}_{3}\right)$ detectada tanto por DRX como por EDX. Además, las micrografías de estas muestras (figura 3 del artículo) mostraron ciertas segregaciones con una morfología muy diferente, que fueron atribuidas a la fase secundaria de perovsquita. Por tanto, se determinó que el límite de la disolución sólida fue de 0.15 cuando la estructura no presenta terbio. Sin embargo, en las muestras que contenían terbio el límite de disolución sólida fue de 0.35 . De 
las evidencias anteriores, se puso de manifiesto que la presencia del terbio juega un papel fundamental, en la incorporación del hierro en la estructura.

Con el objetivo de caracterizar ópticamente el pigmento, así como de estudiar los estados de oxidación del terbio en el mismo, se realizaron medidas de absorción a través de espectrofotometría ultravioleta-visible (UV/Vis), así como de espectroscopia de fotoluminiscencia (PL). En las medidas de UV/Vis, se identificó la presencia de una banda de absorción alrededor de $400 \mathrm{~nm}$ en las muestras que contenían terbio. Esta banda fue atribuida a la transferencia de carga $\mathrm{Tb}^{4+} \rightarrow \mathrm{O}^{2-}$, la cual tiene una repercusión en el color final de la muestra, presentando una coloración roja-naranja más elevada.

Además, las medidas de fotoluminiscencia realizadas permitieron estudiar de una forma más exhaustiva el efecto del terbio en la estructura. A través de estas se determinó que, a mayor cantidad de hierro en la estructura, la cantidad de iones $\mathrm{Tb}^{3+}$ es menor y por consiguiente mayor será la cantidad de $\mathrm{Tb}^{4+}$ (figura 5 del artículo). Este hecho está relacionado con los resultados obtenidos asociados a la presencia de $\mathrm{Tb}^{4+}$. También se realizaron medidas de espectroscopia de fotoelectrones emitidos por rayos X (XPS), con el objetivo de determinar el estado de oxidación del hierro. En la única muestra sin terbio que presentaba fase única, (YF015) el estado de oxidación presente en el hierro fue +3 mayoritariamente. En cambio, en la muestra con la misma cantidad de hierro (YTF015) pero en presencia de terbio, el estado de oxidación mayoritario fue +2 . A partir de este resultado y respaldados con las medidas de fotoluminiscencia se formuló un hipotético mecanismo, para explicar el papel del terbio, como se observa en la ecuación 13.

$$
\begin{gathered}
\mathrm{Tb}^{3+} \rightarrow \mathrm{Tb}^{4+}+1 \mathrm{e}^{-} \\
\mathrm{Fe}^{3+}+1 \mathrm{e}^{-} \rightarrow \mathrm{Fe}^{2+}
\end{gathered}
$$


La presencia de terbio en la estructura promueve la reducción del hierro presente en la misma. Esta tendencia se pone de manifiesto en las coloraciones de las disoluciones sólidas finales, ya que los estados de oxidación están directamente relacionados con el color.

Para ello se midieron las coordenadas cromáticas de las composiciones que presentaban fase única (YF015, YTF015, YTF025, YTF035). Todas las muestras presentaron una buena coloración rojiza. No obstante, aquellas que presentaban mejores coordenadas cromáticas eran las que contenían mayor cantidad de hierro. Esto es debido a que sólo una parte del hierro es capaz de reducirse a $\mathrm{Fe}^{2+}$, siguiendo el mecanismo descrito en la ecuación 13, ya que la cantidad de este es mayor que la del terbio. Por tanto, la muestra con una mayor coloración es la que presenta una mayor cantidad de $\mathrm{Fe}^{3+}$ y $\mathrm{Tb}^{4+}$ (YTF035), ambos asociados a una coloración rojiza y anaranjada respectivamente.

Con el propósito de estudiar su estabilidad en vidrio, las dos composiciones que presentaron mejor coloración fueron mezcladas con un esmalte cerámico. Ambas mostraron una buena estabilidad, presentando YTF035 una coloración rojiza mejor que el pink coral (pigmento industrial utilizado en la actualidad), tratado en las mismas condiciones (figura 7 del artículo). Ambas composiciones también fueron evaluadas para su potencial aplicación como "cool pigments". Para ello se mezclaron con una pintura de siloxano y se midió su reflectancia en la zona del infrarrojo cercano. Para la muestra YTF025 se obtuvieron valores muy satisfactorios de alrededor del $70 \%$ de reflectancia. Además, también se evaluó su rendimiento como "cool pigment" de forma experimental. Para ello se recubrieron pequeñas estructuras de espuma con la pintura de siloxano mezclada con un $12 \%$ del pigmento y otra sin mezclar, ambas se expusieron a radiación infrarroja. Se realizaron medidas de temperatura del aire del interior a diferentes tiempos de exposición con un termopar de baja temperatura. Con este experimento se concluyó que la aplicación de la pintura con el pigmento sintetizado evitaba un aumento de 
la temperatura de hasta $1.3{ }^{\circ} \mathrm{C}$ después de la exposición a una lámpara de infrarroja durante 30 minutos. Por lo tanto, los valores obtenidos de reflectancia en el NIR, así como los estudios experimentales realizados, hacen de este pigmento potencialmente utilizable en la industria de los revestimientos como aislante.

En resumen, se sintetizó un pigmento cerámico de coloración rojiza sin el uso de elementos tóxicos en su estructura. Este pigmento presentó una mejor coloración rojiza que el pink coral en esmalte, siendo altamente reproducible su síntesis mediante la metodología sol-gel polimérica. Además, presenta otro tipo de potenciales aplicaciones en el campo de los aislantes térmicos como "cool pigment", hecho que comportaría un ahorro de grandes cantidades de energía, por ejemplo en la refrigeración de los edificios. 


\subsection{Conclusiones del artículo científico 1}

- Se han optimizado los parámetros de síntesis de la ruta sol-gel polimérico y del posterior tratamiento térmico para la formación de disoluciones sólidas basadas en la estequiometría $\mathrm{Y}_{2} \mathrm{Zr}_{2} \mathrm{O}_{7}$, dopada con hierro y/o terbio.

- Se han determinado los límites de disolución sólida en todas las muestras sintetizadas. En ausencia de terbio solamente es posible incorporar una cantidad de hierro de 0.15. En cambio, fue posible la incorporación de hasta una cantidad de hierro de 0.35 en presencia de 0.25 de terbio. Todas las disoluciones sólidas presentaron una estructura de fluorita defectuosa.

- Se ha corroborado experimentalmente el papel fundamental que juega el terbio en la incorporación del hierro en la estructura, permitiendo una mayor incorporación de éste en la disolución sólida, sin la aparición de fases secundarias.

- Se ha formulado un mecanismo para explicar los estados de oxidación de los cromóforos y la influencia directa de estos en la coloración final presentada por el pigmento. De ello se concluyó, que la presencia de mayores cantidades tanto de iones $\mathrm{Fe}(\mathrm{III})$, así como de iones $\mathrm{Tb}(\mathrm{IV})$, desarrollan una mejor coloración de la estructura pigmentante.

- Se han comparado las coordenadas cromáticas en esmalte de los pigmentos sintetizados, con las coordenadas del pink coral (pigmento rojizo utilizado industrialmente). La disolución sólida $\mathrm{Y}_{1.75} \mathrm{~Tb}_{0.25} \mathrm{Zr}_{1.65} \mathrm{Fe}_{0.35} \mathrm{O}_{6.825}$ (YTF035), 
presentó mejores coordenadas en esmalte que el pink coral, exhibiendo una tonalidad más rojiza.

- Se ha comprobado el potencial usó de los pigmentos sintetizados en el campo de los denominados "cool pigments". Para ello se realizaron medidas de reflectancia tanto en polvo como de la mezcla del pigmento en pintura. La composición $\mathrm{Y}_{1.75} \mathrm{~Tb}_{0.25} \mathrm{Zr}_{1.75} \mathrm{Fe}_{0.25} \mathrm{O}_{6.875}$ (YTF025) presentó valores de reflectancia en el infrarrojo cercano muy satisfactorios, alrededor del $90 \%$ en polvo y del $70 \%$ en pintura.

- Se ha evaluado la potencial aplicación del pigmento como "cool pigment" experimentalmente, minimizando el aumento de la temperatura del aire del interior de una estructura recubierta con la pintura y el pigmento de hasta $1.3^{\circ} \mathrm{C}$ después de la exposición a una lámpara infrarroja durante 30 minutos.

Por lo tanto, se ha sintetizado un pigmento de coloración rojiza, sin el uso de elementos tóxicos, con una mejor coloración en esmalte que el pigmento rojo utilizado industrialmente. Además, presenta propiedades multifuncionales, ya que también puede ser utilizado en el área de los denominados "cool pigments", por sus propiedades ópticas, obteniendo unos valores de reflectancia en el infrarrojo cercano de alrededor del $70 \%$ en pintura. 


\section{CAPÍTULO 4}

\section{Síntesis del pigmento de coloración roja-naranja basado en $\mathrm{Y}_{2} \mathrm{Zr}_{2} \mathrm{O}_{7}$ dopado con Pr y Fe}

"Creemos que no hay color, pensamos que no es dulce, pensamos que no es amargo, pero en realidad hay átomos y el vacio" 



\subsection{Introducción}

En el presente capítulo, se aborda la síntesis de un nuevo pigmento cerámico basado en una estructura fluorita defectuosa con estequiometría $\mathrm{Y}_{2} \mathrm{Zr}_{2} \mathrm{O}_{7}$ dopado con praseodimio en la posición del itrio y hierro en la posición del circonio.

El praseodimio ha sido uno de los lantánidos usados históricamente en el campo de los pigmentos cerámicos. Un claro ejemplo de su uso, es el pigmento amarillo de praseodimio-circón [DCMA-14-43-2 ( $\left.\mathrm{Pr}-\mathrm{ZrSiO}_{4}\right)$ ], el cual es uno de los pigmentos cerámicos más producidos en la industria de los pigmentos cerámicos en los últimos años, debido a su nula toxicidad y su amplio intervalo de propiedades ópticas [66]. La formación de esta estructura fue documentada por primera vez por Fuji y Sono [149]. Desde entonces han sido muchos los estudios realizados sobre esta estructura pigmentante, su síntesis por diferentes métodos, el estudio de los precursores óptimos, así como estudios del mecanismo de coloración en dicho pigmento [150-154]. Además, existen diversos estudios en los cuales se combinan el praseodimio con otros cromóforos tales como el cerio o el hierro [155,156].

Por lo tanto, la combinación de hierro y praseodimio en una misma estructura parece una buena estrategia con el objetivo de desarrollar un nuevo pigmento cerámico sin la presencia de cromóforos tóxicos. Por esta razón, en el presente capítulo se estudia la posibilidad de preparar un nuevo pigmento rojizo basado en $\mathrm{Y}_{2} \mathrm{Zr}_{2} \mathrm{O}_{7}$ sustituyendo parcialmente al circonio por hierro, y el praseodimio sustituyendo parcialmente al itrio. Se estudiaron a su vez los posibles mecanismos de coloración de dicho pigmento. 
El praseodimio en estado de oxidación +3 igual que el terbio en este mismo estado de oxidación, exhibe propiedades luminiscentes. Como el resto de los lantánidos presenta la suma de las tres primeras entalpias de ionización relativamente baja, por lo que es fuertemente electropositivo, formando iones $\operatorname{Pr}^{3+}$ $\left(4 f^{2}\right)$ siendo este estado de oxidación más frecuente, aunque también se pueden producir iones $\operatorname{Pr}^{4+}\left(4 f^{1}\right)$. Esta característica, hace del praseodimio un elemento óptimo para su uso en una amplia gama de materiales luminiscentes, tales como láseres y fósforos [157-161].

El dopaje con praseodimio (III) en óxidos, proporcionará bandas de luminiscencia en la región azul-verde del espectro $\left({ }^{3} \mathrm{P}_{0} \rightarrow{ }^{3} \mathrm{H}_{4}\right)$ y roja $\left({ }^{1} \mathrm{D}_{2} \rightarrow{ }^{3} \mathrm{H}_{4}\right)$, con diferentes intensidades [162]. Estas bandas dependerán fuertemente de la concentración de praseodimio en la estructura, así como de la longitud de onda utilizada en la excitación. Estas emisiones fotolumiscentes son debidas a la desexcitación al estado fundamental $\left({ }^{3} \mathrm{H}_{4}\right)$ de un electrón, previamente excitado a estados superiores de una forma radiativa, con la emisión de un fotón. La figura 15 muestra esquemáticamente el diagrama de niveles energéticos del $\mathrm{Pr}^{3+}$, así como las emisiones correspondientes. Las transiciones dependen de la longitud de onda de excitación, así como de la red huésped en la que se encuentran. Además, existen estudios, donde el praseodimio sustituye al átomo de circonio en la circona, que muestran la existencia de un proceso de relajación del material a través de una transferencia de carga entre la red huésped $\mathrm{ZrO}_{2}$ y las bandas $4 f 5 d$ o los niveles $4 f$ del $\operatorname{Pr}^{3+}$ [163]. Respecto a la composición que en la presente tesis nos atañe, $\mathrm{Y}_{2} \mathrm{Zr}_{2} \mathrm{O}_{7}$, existen estudios de su dopaje con diferentes iones lantánidos, entre ellos el praseodimio, para sus utilización exclusiva cómo fósforos [65,111,113,164]. Estas propiedades luminiscentes de los iones $\operatorname{Pr}^{3+}$, son de gran utilidad no sólo en el campo de los materiales luminiscentes y/o pigmentos luminiscentes, sino también en el campo de los pigmentos cerámicos, ya que proporcionan una gran ayuda en el 
estudio de las variaciones los estados de oxidación de los cromóforos y su influencia en el color.

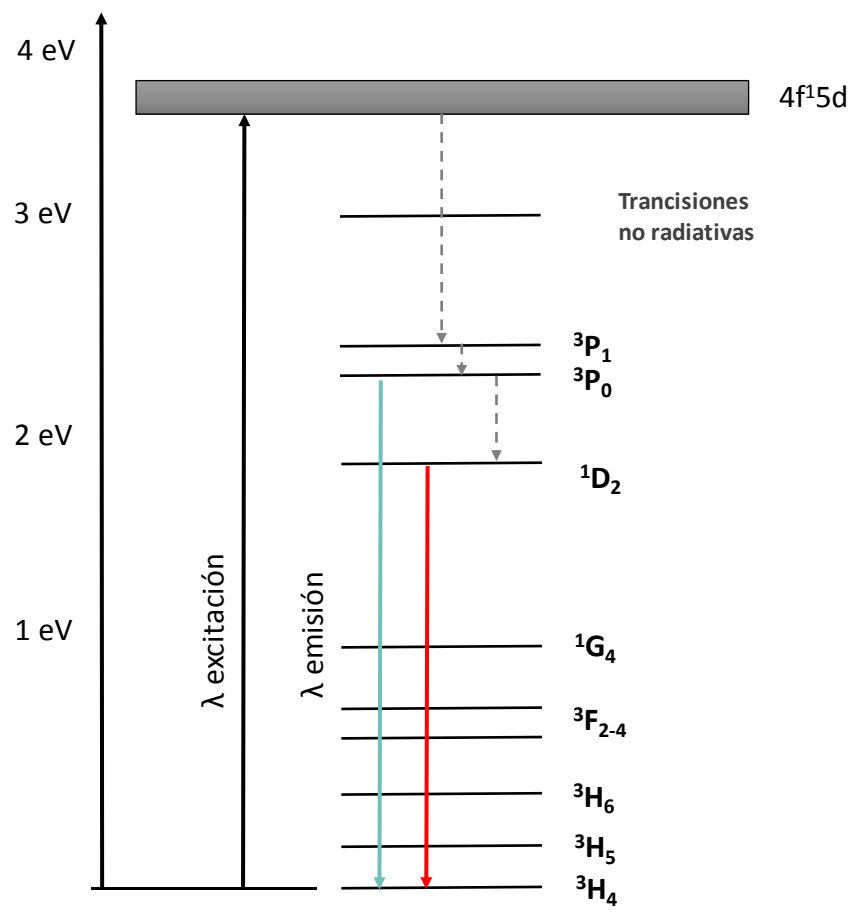

Figura 15. Diagrama de niveles energéticos y transiciones del $\operatorname{Pr}^{3+}$.

Además, como se ha visto en el capítulo anterior, el cambio de los estados de oxidación del lantánido, puede influir fuertemente en el estado de oxidación de los otros elementos cromóforos, y en consecuencia una variación del color final [165]. Asimismo, el elemento lantánido dopante podría actuar como "sonda" para poder predecir el estado de oxidación del otro cromóforo.

Por lo que se refiere al praseodimio (IV), este no presenta luminiscencia, sin embargo, se le atribuye una transferencia de carga de los iones óxido al nivel $4 f^{1}$ del $\mathrm{Pr}^{4+}$, así como las transiciones propias entre los niveles energéticos $4 f$ y $5 d$. Esta 
conlleva una banda ancha de absorción en el intervalo 330-590 nm teniendo una fuerte influencia en la coloración final del pigmento [153,166,167].

El conocimiento de los mecanismos de coloración en los pigmentos, es básico para una correcta optimización de las condiciones de síntesis y una mejora de las propiedades de los pigmentos ya existentes, con el objetivo de obtener el máximo rendimiento de los precursores utilizados, y también es de vital importancia en el desarrollo de nuevas estructuras pigmentantes. Siguiendo esta tendencia, son muchas las investigaciones existentes con el principal objetivo de conocer que provoca que un pigmento tenga uno u otro color $[168,169]$.

Tal y como se ha realizado en el capítulo anterior existen varias estrategias y técnicas para conocer el estado de oxidación de los elementos. Una de las técnicas más potentes en este sentido es la espectrometría fotoelectrónica de rayos X (XPS). Esta técnica es ampliamente utilizada para la determinación del estado de oxidación del hierro tanto en óxidos como en otro tipo de estructuras [170-173]. Por lo tanto, se disponen de las herramientas necesarias para poder conocer los estados de oxidación de los dos dopantes con los que se va a dopar el compuesto $\mathrm{Y}_{2} \mathrm{Zr}_{2} \mathrm{O}_{7}$, la fotoluminiscencia para el praseodimio y el XPS para el hierro.

Como se ha puesto de manifiesto en capítulos anteriores, la industria de los pigmentos actual, no sólo busca el desarrollo de color en esmaltes cerámicos, sino también la aplicación en otro tipo de medios, tales como polímeros o pinturas, para el desarrollo de color $[109,174,175]$. La aplicación de un pigmento en estos medios diferentes al esmalte tradicional, es altamente demandada y estudiada debido a las propiedades adicionales que el pigmento es capaz de proporcionar a la pintura o el polímero, lo cual le permitirá adquirir nuevas funcionalidades. En este sentido el desarrollo de pigmentos con un alta reflectancia en la zona del infrarrojo cercano (NIR), es un campo en crecimiento [109, 176-178]. 
En el campo de los pigmentos con una alta reflectancia en el infrarrojo, se plantean los mismos problemas de toxicidad que en el de los pigmentos cerámicos tradicionales, ya que la mayoría de los denominados "cool pigments" contiene elementos pesados, altamente tóxicos tales como el $\mathrm{Cr}, \mathrm{Cd}, \mathrm{Ni}, \mathrm{Pb}$, etc., cuyo uso está restringido en muchos países [179].

Por lo tanto, la posibilidad de desarrollar color en diferentes medios y las nuevas funcionalidades que la mezcla con el pigmento les aporta, le confieren al pigmento una multifuncionalidad y en definitiva un valor añadido, al desarrollo del propio color, haciéndolo multifuncional y en consecuencia mucho más atractivo.

El presente trabajo de investigación se basa en la síntesis mediante el método sol gel composiciones basadas en $\mathrm{Y}_{2-\mathrm{x}} \mathrm{Pr}_{\mathrm{x}} \mathrm{Zr}_{2-\mathrm{y}} \mathrm{Fe}_{\mathrm{y}} \mathrm{O}_{7-\delta}$ con diferentes valores de $\mathrm{x}=0.15,0.25,0.35$ e y $=0.15,0.25,0.35$. Se realizó un estudio detallado de los estados de oxidación de ambos cromóforos relacionándolos con la coloración final tanto en polvo como en esmalte. A partir de estos resultados se propuso un posible mecanismo de coloración.

Además, se estudió la estabilidad del pigmento en esmalte, pintura y PMMA estudiando su potencial uso como pigmento refrigerante o "cool pigment" en este tipo de matrices. 


\subsection{Objetivos del artículo científico 2}

Los objetivos generales de este trabajo son:

- Optimizar los parámetros de síntesis para la síntesis vía sol-gel polimérico de un pigmento cerámico de coloración rojiza basado en la estequiometría $\mathrm{Y}_{2} \mathrm{Zr}_{2} \mathrm{O}_{7}$ dopado con hierro y praseodimio.

- Establecer los límites de la disolución sólida en la estructura $\mathrm{Y}_{2-\mathrm{x}} \mathrm{Pr}_{\mathrm{x}} \mathrm{Zr}_{2-\mathrm{y}} \mathrm{Fe}_{\mathrm{y}} \mathrm{O}_{7-\delta}$ con valores de $\mathrm{x}=0.15,0.25,0.35$ e $\mathrm{y}=0.15,0.25,0.35$.

- Estudiar los estados de oxidación de los cromóforos, así como su influencia en el mecanismo de coloración del pigmento.

- Comprobar la estabilidad del pigmento en diferentes dispersantes tales como esmalte cerámico, pintura y polímero.

- Comprobar el potencial uso del pigmento en el área de los pigmentos fríos o "cool pigments", en diferentes medios de dispersión, tales como el esmalte, pintura o en un polímero. 


\subsection{Artículo científico 2}

Título: Environmental-friendly red-orange ceramic pigment based on $\mathrm{Pr}$ and $\mathrm{Fe}$ co-doped $\mathrm{Y}_{2} \mathrm{Zr}_{2} \mathrm{O}_{7}$

Revista: Journal of the European Ceramic Society

Autores: Marc Jovaní, Maria Fortuño Morte, Héctor Beltrán-Mir, Eloisa Cordoncillo.

Índice de Impacto: 3.794

Revista dentro del $25 \%: \mathrm{Si}$

Categoría: Ciencia de materiales cerámicos

Posición: $1 / 26$ 

Original Article

\title{
Environmental-friendly red-orange ceramic pigment based on $\mathrm{Pr}$ and $\mathrm{Fe}$ co- doped $\mathrm{Y}_{2} \mathrm{Zr}_{2} \mathrm{O}_{7}$
}

\author{
Marc Jovaní, Maria Fortuño-Morte, Héctor Beltrán-Mir*, Eloísa Cordoncillo \\ Departamento de Química Inorgánica y Orgánica, Universitat Jaume I de Castellón, Av. Sos Baynat s/n, 12071, Castelló de la Plana, Spain
}

\section{A R T I C L E I N F O}

\section{Keywords:}

Red-orange pigment

Environmental-friendly

Sol-gel

Defect fluorite

\begin{abstract}
A B S T R A C T
A new environmental-friendly red-orange ceramic pigment based on the general formula $\mathrm{Y}_{2-\mathrm{x}} \mathrm{Pr}_{\mathrm{x}} \mathrm{Zr}_{2-\mathrm{y}} \mathrm{Fe}_{\mathrm{y}} \mathrm{O}_{7-\delta}$ was prepared by polymeric sol-gel method at $1400{ }^{\circ} \mathrm{C}$. The solid solution range with defect fluorite structure was determined by XRD and SEM/EDX. The oxidation states of the dopants were studied by UV/Vis absorption measurements, XPS and photoluminescence, and a possible redox mechanism was proposed. The colour of the pigment was determined by CIEL*a*b* chromatic coordinates in powder, glass, paint and polymer. All compositions presented a good stability in powder and in glass, the $\mathrm{Y}_{1.75} \mathrm{Pr}_{0.25} \mathrm{Zr}_{1.75} \mathrm{Fe}_{0.25} \mathrm{O}_{6.875}$ composition being the best red good colouration. This composition also showed an optimal NIR reflectance $80 \%$ in paint to be applied as "cool pigment" for roof isolation. The solid solutions were also tested in PMMA showing a good stability in the sunlight. Therefore, a red-orange environmental-friendly pigment was synthesised with potential applications in the field of the ceramic tiles, cool roofing materials and polymers.
\end{abstract}

\section{Introduction}

The minimization of the human footprint on the earth is one of the most important challenges in the modern world. The ceramic pigments industry is no stranger to this trend; therefore, the research in this way is constantly increasing. A wide range of ceramic pigments contains toxic elements as chromophores. Nowadays, some alternatives are shown in literature to avoid the use of them [1-7]. Due to the low toxicity and their unique optical properties, lanthanides are included as promising elements in the inorganic pigments field. These elements can avoid the use of chromophores of high toxicity like cobalt, chromium or cadmium that are an important source of pollution $[5,8]$.

The environmental problem concerns all ceramic pigments, but the use of toxic chromophores is highlighted in red ceramic pigments [9]. Until now, the iron doped zircon pigment $\left(\mathrm{Fe}-\mathrm{ZrSiO}_{4}\right)$, commonly called pink coral, was a good alternative in order to develop red shade pigments environmentally friendly for the ceramic industry [10], but this pigment presents other kinds of problems. For example, the colour of the pink coral is difficult to control compared to other inorganic pigments. There must be very precise fine grinding of the starting materials (zirconia and silica) to obtain the desirable colour. Finer particles can give a better coral pink colour that is more reddish and yellowish in tone. As a consequence, this pigment has a significant problem of reproducibility and the final colour of the powder can vary widely $[11,12]$. Moreover, the colouring mechanism is not understood. The location of the iron and its valence in the zircon lattice is unclear. In spite of the controversial conclusions that there are in the literature $[10,12,13]$, it is generally accepted that the oxidation state of iron is three, but there exists certain disagreement about the role played by iron in the formation of the zircon lattice, and its position in the structure like interstitial or substitutional ion $[10,14]$.

Iron is commonly used as a chromophore ion in ceramic pigments $[10,15-18]$. The knowledge of the oxidation state of the iron is fundamental to understand the colouring mechanism of the ceramic pigment and to improve the colouration. Other dopant chromophore elements as the lanthanides can be used to change the original oxidation state of the iron and as a consequence the colour. The presence of these in the pigmenting structures not only plays the role of the chromophore, but also plays the role of the modifier of the oxidation state of others components of the lattice, modifying the crystal field, affecting greatly the final colour of the powders. In this sense, in a previous work, studying a pigment based on $\mathrm{Y}_{2-\mathrm{x}} \mathrm{Tb}_{\mathrm{x}} \mathrm{Zr}_{2-\mathrm{y}} \mathrm{Fe}_{\mathrm{y}} \mathrm{O}_{7-\delta}$ solid solution, authors observed that a variation in the oxidation state of terbium from $\mathrm{Tb}(\mathrm{III})$ to $\mathrm{Tb}(\mathrm{IV})$ produced the reduction of the $\mathrm{Fe}(\mathrm{III})$ to $\mathrm{Fe}(\mathrm{II})$. And also showed that this fact had a great influence in the final colour of the powders, changing the intensity of the red colour obtained [6].

For this work, praseodymium and iron doped $\mathrm{Y}_{2} \mathrm{Zr}_{2} \mathrm{O}_{7}$ was selected in order to study the influence of the oxidation state in the final colour of the pigment. This general structure, $\mathrm{A}_{2} \mathrm{~B}_{2} \mathrm{O}_{7}$, containing trivalent $\mathrm{A}$ and tetravalent $\mathrm{B}$ cations has been studied recently as a new pigment,

\footnotetext{
* Corresponding author.

E-mail address: mir@uji.es (H. Beltrán-Mir).
} 
due to its compositional versatility and high stability $[8,19]$. Compounds with $\mathrm{A}_{2} \mathrm{~B}_{2} \mathrm{O}_{7}$ stoichiometry have been studied for their extensive field of applications and also for their ability to incorporate a wide range of chromophore ions in both positions A and B [19]. This structure is optimal in order to develop pure colours due to the possibility of incorporating a great range of chromophores. According to the radius ratios of the ions present [20], $\mathrm{Y}_{2} \mathrm{Zr}_{2} \mathrm{O}_{7}$ acquires a defect fluorite structure under ambient conditions. Defect-fluorite structure presents Fm-3m space group with a random distribution of both cations and anions onto their respective sublattices. Zhang et al. [21] have recently predicted that the coordination of cations in the $\mathrm{Y}_{2} \mathrm{Zr}_{2} \mathrm{O}_{7}$ defect-fluorite varies depending on the species with the average coordination of $\mathrm{Y}$ and $\mathrm{Zr}$ being 7.2 and 6.8, respectively. This crystalline structure is significantly flexible, i.e., the lattice can stabilize large amounts of ionic substitutions in each one of its crystalline sites, as well as vacancies and structural defects.

Praseodymium is also used in a widely variety of structures with different applications like pigments or laser [22-26]. Among all these applications, in the field of the ceramic pigments praseodymium is present in a great variety of environmental-friendly pigments due to their optical properties $[3,4,27]$. Like the rest of the lanthanides, the praseodymium exhibits the first three ionization energies relatively low, being strongly electropositive acquiring the +3 oxidation state easily $\left(4 f^{2}\right)$. This is the most frequent oxidation state of this element. But it is also presented in the oxidation state $+4\left(4 f^{1}\right)$ being the mixture of both oxidation states $+3 /+4$ are also very frequent. This behaviour become the praseodymium an optimal chromophore due to the strong absorptions of $\mathrm{Pr}^{3+}$ in the blue and red regions as a consequence of the electronic transitions between $4 f^{2} \rightarrow 5 d^{1}$ states of $\mathrm{Pr}^{3+}$. However, the highly forbidden $4 f \rightarrow 4 f$ transitions of $\mathrm{Pr}^{+3}$ are practically without influence on the colouration. While the oxidation state +4 is related with the increase of the yellow colouration due to the different charge transfer bands of $\operatorname{Pr}^{+4}[28,29]$.

The development of new solids with interesting colour applications is an attractive topic for researchers and industry. The knowledge of the colouration mechanism in ceramic pigments is fundamental in order to develop new colourations and optimize the synthesis parameters and its precursors. This study is one of the main goals of this study. The colouring mechanism of the $\mathrm{Pr}$ and $\mathrm{Fe}$ doped $\mathrm{Y}_{2} \mathrm{Zr}_{2} \mathrm{O}_{7}$ system was studied, but also the potential application of this material in glass, paints and polymers as a multifunctional pigment was also tested.

Over the last years, one of the most interesting applications of the inorganic pigments is cool roof coatings with high near infrared reflectance (NIR) [30-32]. White pigments, mainly $\mathrm{TiO}_{2}$ [33], with high solar reflectance are often used as cool materials, but other coloured pigments have been developed because of the necessary aesthetic requirements. Cool pigments are used as coatings on building roofs in order to decrease the temperature in the interior of the house, and consequently, contribute to increased indoor thermal comfort levels in hot seasons, resulting in reducing the need for cooling [34]. Many of these coloured pigments with high NIR reflectivity also contain toxic metal elements like $\mathrm{Co}, \mathrm{Pb}, \mathrm{Cd}$ or $\mathrm{Cr}$. These are restricted by the current environmental regulations [35-37]. In this way, some new potential cool pigments have been also reported in order to substitute these traditional toxic elements $[6,38,39]$.

Another interesting application is the use of the ceramic pigments as a plastic colourant [40-44]. Regarding pigments for polymeric material, the main characteristics required are insolubility, high polymer dispersion, intense colour, and high colouring ability $[42,45]$. When opacity and higher stability regarding exposure to light and heating is desired, these pigments are also used. In addition, inorganic pigments present higher stability and lower migration effect when they are in contact with chemical products, and they are used to substitute organic pigments [45].

Thus, this work reports the preparation and the study of colouring mechanism of a novel red-orange $\mathrm{Y}_{2-\mathrm{x}} \mathrm{Pr}_{\mathrm{x}} \mathrm{Zr}_{2-\mathrm{y}} \mathrm{Fe}_{\mathrm{y}} \mathrm{O}_{7-\delta}$ system prepared
Table 1

Compositions prepared based on $\mathrm{Y}_{2-\mathrm{x}} \mathrm{Pr}_{\mathrm{x}} \mathrm{Zr}_{2-\mathrm{y}} \mathrm{Fe}_{\mathrm{y}} \mathrm{O}_{7-\delta}$.

\begin{tabular}{ll}
\hline Reference & Composition \\
\hline P015F025 & $\mathrm{Y}_{1.85} \mathrm{Pr}_{0.15} Z_{1.75} \mathrm{Fe}_{0.25} \mathrm{O}_{6.875}$ \\
P025F025 & $\mathrm{Y}_{1.75} \mathrm{Pr}_{0.25} \mathrm{Zr}_{1.75} \mathrm{Fe}_{0.25} \mathrm{O}_{6.875}$ \\
P035F025 & $\mathrm{Y}_{1.65} \mathrm{Pr}_{0.35} \mathrm{Zr}_{1.75} \mathrm{Fe}_{0.25} \mathrm{O}_{6.875}$ \\
P025F015 & $\mathrm{Y}_{1.75} \mathrm{Pr}_{0.25} \mathrm{Zr}_{1.85} \mathrm{Fe}_{0.15} \mathrm{O}_{6.925}$ \\
P025F035 & $\mathrm{Y}_{1.75} \mathrm{Pr}_{0.25} \mathrm{Zr}_{1.65} \mathrm{Fe}_{0.35} \mathrm{O}_{6.825}$ \\
\hline
\end{tabular}

by the sol-gel method. The multifunctional features of the pigment were studied and evaluated in glass, paint and polymer.

\section{Experimental}

Two different groups of compositions based on the general formula $\mathrm{Y}_{2-\mathrm{x}} \mathrm{Pr}_{\mathrm{x}} \mathrm{Zr}_{2-\mathrm{y}} \mathrm{Fe}_{\mathrm{y}} \mathrm{O}_{7-\delta}$, Table 1, were prepared by polymeric sol-gel procedure. In a first group, praseodymium content is fixed and the amount of iron is changing, while in a second group the praseodymium is changing with fix iron content. $\mathrm{Y}\left(\mathrm{OOCCH}_{3}\right)_{3} \cdot \mathrm{H}_{2} \mathrm{O}(99.9 \%$, Strem Chemicals), $\mathrm{Zr}\left(\mathrm{OCH}_{2} \mathrm{CH}_{2} \mathrm{CH}_{3}\right)_{4} \quad\left(70 \%\right.$ Sigma-Aldrich), $\operatorname{Pr}\left(\mathrm{NO}_{3}\right)_{3} \cdot 6 \mathrm{H}_{2} \mathrm{O}$ (99.9\% Acros Organics), and $\mathrm{Fe}\left(\mathrm{NO}_{3}\right)_{3} \cdot 9 \mathrm{H}_{2} \mathrm{O}$ (98\% Strem Chemicals) with an analytical grade and without purification were used as precursors. Absolute ethanol (Scharlab, 99.9\%) was used as a solvent. A scheme of the general preparation of the samples is shown in Fig. 1. Zirconium precursor was dissolved in ethanol. Then $\mathrm{Y}\left(\mathrm{OOCCH}_{3}\right)_{3} \cdot \mathrm{H}_{2} \mathrm{O}$, $\mathrm{Fe}\left(\mathrm{NO}_{3}\right)_{3} \cdot 9 \mathrm{H}_{2} \mathrm{O}$ and $\operatorname{Pr}\left(\mathrm{NO}_{3}\right)_{3} \cdot 6 \mathrm{H}_{2} \mathrm{O}$ were added, and the mixture was stirred for $10 \mathrm{~min}$. This mixture was transferred into a balloon flask and, heated at $70{ }^{\circ} \mathrm{C}$ for $72 \mathrm{~h}$. A gel was formed and it was dried in air at room temperature. Finally, the powder was fired at $1400{ }^{\circ} \mathrm{C}$ for $12 \mathrm{~h}$.

Powders of samples with optimal colour were milled and sieved at

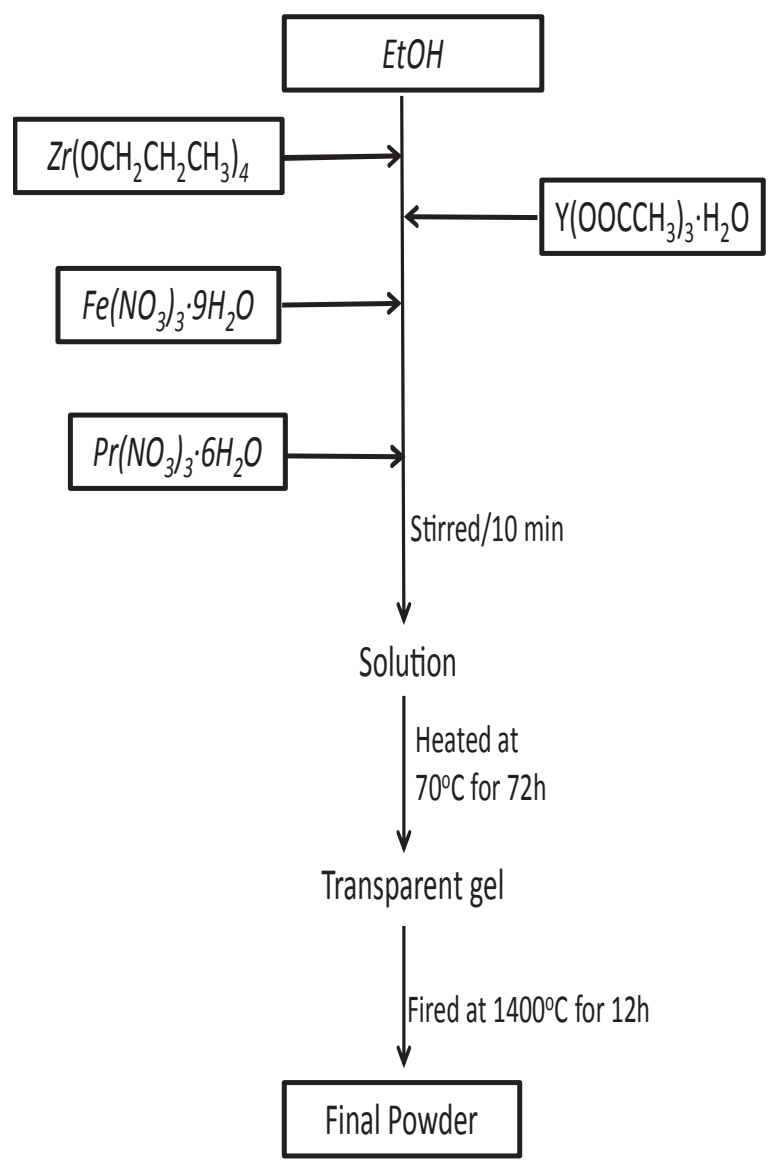

Fig. 1. Scheme for the synthesis procedure. 
Table 2

Frit composition.

\begin{tabular}{lccccc}
\hline \multicolumn{2}{l}{ Composition $(w t \%)^{\mathrm{a}}$} & & & & \\
\hline $\mathrm{SiO}_{2}$ & $\mathrm{Al}_{2} \mathrm{O}_{3}$ & $\mathrm{RO}^{\mathrm{b}}$ & $\mathrm{R}_{2} \mathrm{O}^{\mathrm{b}}$ & $\mathrm{ZnO}$ & $\mathrm{ZrO}_{2}$ \\
\hline 67 & 13 & 9.4 & 10 & 0.4 & 0.2 \\
\hline
\end{tabular}

a The percentages do not represent quantitative analyses.

${ }^{\mathrm{b}} \mathrm{R}=$ alkaline or alkaline earth metals.

$0.06 \mathrm{~mm}$, and mixed by ball milling with commercial transparent frit ( $4 \%$ in weight of the pigment) using water as a dispersing medium. The frit composition used is given in Table 2. Then, the dispersion was applied to white twice-fired bodies, to verify composition stability as a ceramic colourant. After drying, the pieces were fired in an electric kiln. The heat treatment applied, corresponds to a standard firing cycle used in a ceramic tile industry where the highest temperature of the cycle was $1080^{\circ} \mathrm{C}$ for $5 \mathrm{~min}$. This cycle involves five steps: ramping to $300{ }^{\circ} \mathrm{C}$ in $10 \mathrm{~min}$, heating from $300^{\circ} \mathrm{C}$ to glaze firing temperature in $17 \mathrm{~min}$, 5 min hold at $1080{ }^{\circ} \mathrm{C}$, cooling to $600{ }^{\circ} \mathrm{C}$ in $20 \mathrm{~min}$, and finally cooling to room temperature in $15 \mathrm{~min}$.

For the test of samples as cool pigments, the prepared pigments were mixed with siloxane transparent paint $(12 \%$ in weight of the pigment) and applied to a ceramic tile $(8 \mathrm{~cm} 8 \mathrm{~cm})$. Besides, these pigments were mixed with poly(methylmethacrylate) (PMMA) ( $\gg>99 \%$, Beuhler) (10\% in weight of the pigment) in order to test the stability in polymers.

\section{Characterization}

Phase analysis of the samples was performed by powder XRD using a Bruker D8 Advance diffractometer with $\mathrm{CuK}_{\alpha}$ radiation. Data were collected by step-scanning from $2 \mathrm{q}=20$ to $70^{\circ}$ with a step size of $0.05^{\circ}$ and $1.5 \mathrm{~s}$ of counting time at each step.

Scanning electron micrographs of the samples were taken on a field emission scanning electron microscope (FE-SEM) JEOL 7001F, equipped with a spectrometer of energy dispersion of X-ray (EDX) from Oxford instruments, using acceleration voltage $=15 \mathrm{kV}$. Semi-quantitative analysis was performed from EDX taking an average of ten measurements in different particles. Samples for microstructures and microanalysis determinations were deposited in an aluminium holder and sputtered by platinum.

UV-visible (UV/Vis) diffuse reflectance spectroscopy and colourimetric study of the samples were performed on a CARY 500 SCAN VARIAN spectrophotometer in the $380-750 \mathrm{~nm}$ range. $\mathrm{BaSO}_{4}$ was used as a reference. The CIELab colour parameters $L^{*}, a^{*}$, and $b^{*}$ of the samples were determined by coupling analytical software for colour measurements to the VARIAN spectrophotometer, using a standard illuminant D65, to differentiate the pigment in terms of colour. $\mathrm{L}^{*}$ is the lightness axis [black $(0)$ to white $(100)$ ], $a^{*}$ is the green $(\ll 0)$ to red $(\gg>0)$ axis, and $b^{*}$ is the blue $(\ll 0)$ to yellow $(\gg>0)$ axis. Nearinfrared reflectance of powders and paint were performed on the same spectrophotometer in the $800-2400 \mathrm{~nm}$ range.

Photoluminescence (PL) measurements were performed at room temperature using a CARY ECLIPSE VARIAN fluorescence spectrophotometer in the $400-600 \mathrm{~nm}$ range. Emission spectra were excited at $308 \mathrm{~nm}$.

X-ray photoelectron spectroscopy (XPS, K-ALPHA, Thermo Scientific) was used in order to collect the XPS spectra. All spectra were collected using Al- $\mathrm{K}_{\alpha}$ radiation $(1486.6 \mathrm{eV})$, monochromatized by a twin crystal monochromator, yielding a focused X-ray spot (elliptical in shape with a major axis length of $400 \mu \mathrm{m}$ ) at $3 \mathrm{~mA} \mathrm{x} 12 \mathrm{kV}$. The alpha hemispherical analyser was operated in the constant energy mode with survey scan pass energies of $200 \mathrm{eV}$ to measure the whole energy band and $50 \mathrm{eV}$ in a narrow scan to selectively measure the particular
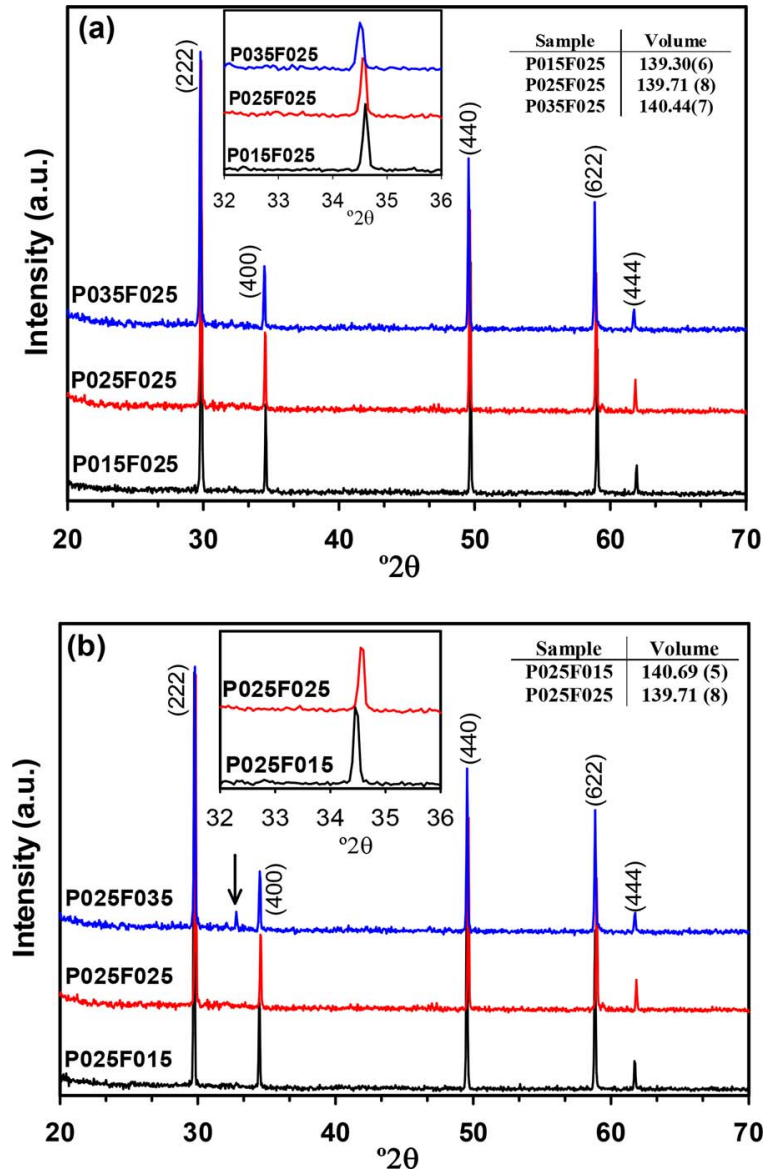

Fig. 2. XRD patterns for powder samples fired at $1400{ }^{\circ} \mathrm{C}$ for $12 \mathrm{~h}$ : (a) $\mathrm{P} 015 \mathrm{~F} 025$, P025F025 and P035F025; (b) P025F015, P025F025 and P025F035.

elements. XPS data were analysed with Avantage software. A smart background function was used to approximate the experimental backgrounds and surface elemental composition was calculated from background-substracted peak areas. Charge compensation was achieved with the system flood gun that provides low energy electrons and low energy argon ions from a single source.

\section{Results and discussion}

XRD patterns of the different compositions fired at $1400{ }^{\circ} \mathrm{C}$ are shown in Fig. 2. Single phase was obtained in all samples except in P025F035. The XRD patterns showed the characteristic peaks of defect fluorite structure [JCPDS-ICDD 49-1642] presenting five characteristic reflection peaks (222), (400), (440), (622) and (444) from low to high $2 \theta$ angles, respectively. The sample with highest amount of iron, presents a secondary phase, marked with an arrow in Fig. 2, that it can attribute to an orthorhombic ferrite phase $\mathrm{YFeO}_{3}$ [JCPDS-ICDD 86-171]. In order to confirm the formation of the solid solution, the displacement of the Bragg peaks in XRD patterns was analysed (insets of Fig. 2a and b). The shifting effect is qualitatively understandable according to Bragg's law $n \lambda=2 d \sin \theta$, where $d$ is the interplanar distance, $\theta$ is the diffraction angle, and $\lambda$ is the diffraction wavelength. A displacement of the diffraction peaks towards lower $2 \theta$ angles is observed when the amount of praseodymium increase and the iron content is fix, inset of Fig. 2a. Based on the radii of $\mathrm{Y}^{3+}(96 \mathrm{pm}, 7 \mathrm{CN})$ and $\mathrm{Pr}^{3+}(99$ pm, $6 \mathrm{CN} ; 112.6 \mathrm{pm}, 8 \mathrm{CN}$ ) [46] if the substitution occurs, the interplanar distance should be increased and therefore, $2 \theta$ angles decreased. According to this, a solid solution was formed in this case. On the other hand, the peaks shifted to higher $2 \theta$ angles when the amount of iron increases and the praseodymium content is fixed, inset of Fig. 2b. For 


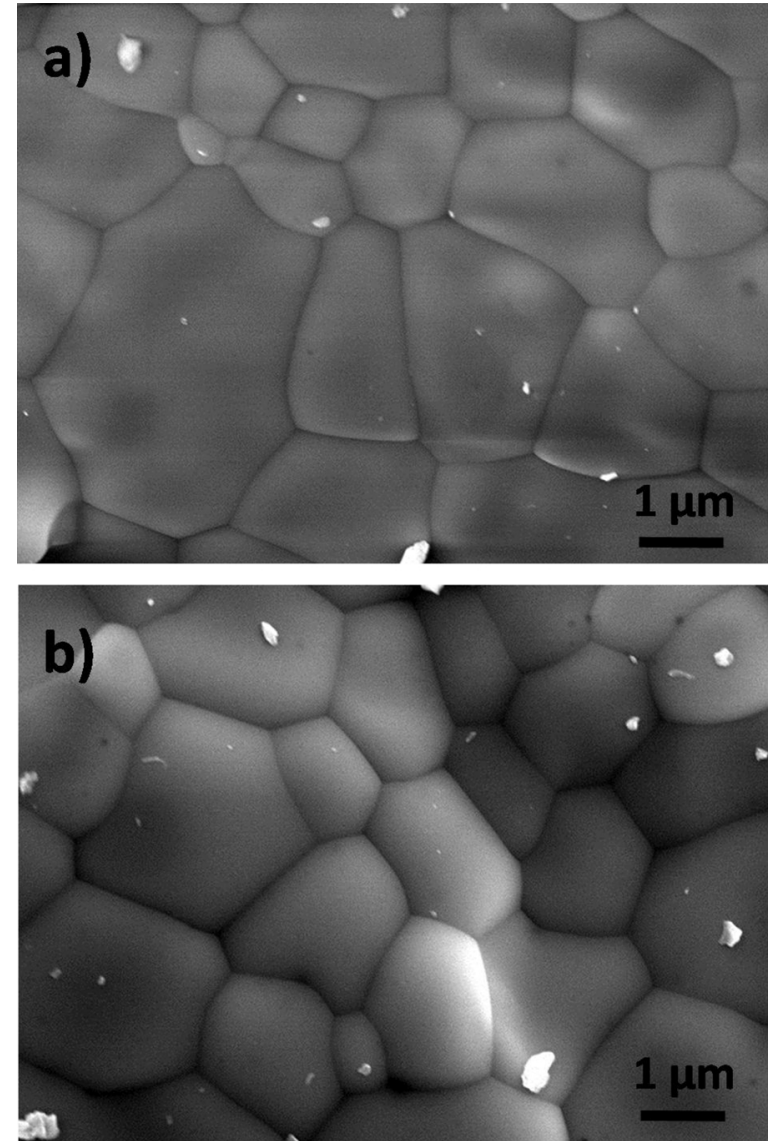

Fig. 3. Micrographs of the powders fired at $1400{ }^{\circ} \mathrm{C}$ a) P025F025 b) P035F025.

the same reason, according to the radii of $\mathrm{Zr}^{4+}(78 \mathrm{pm}, 7 \mathrm{CN})$ and $\mathrm{Fe}^{3+}$ (64.5 pm, $6 \mathrm{CN} ; 78 \mathrm{pm}, 8 \mathrm{CN}$ ) a solid solution also formed. The variation of the volume for all the samples is also shown in the inset of the figures as tables. These variations are also consistent with the ionic radius values of the cations and dopants involved, and demonstrate that Pr and Fe are incorporated into the structure.

In order to analyse the presence of secondary phases not detected by XRD, SEM and EDX analysis were performed in all single-phase samples. Fig. 3 shows the micrographs of the samples with the maximum amount of Fe and Pr on the solid solution, P025F025 and P035F025, respectively. The micrographs showed a good sinterization with welldefined grain boundaries, and grain size was in the range of $0.5-$ $2 \mu \mathrm{m}$. There was no evidence of secondary phases by EDX. In this way, the presence of praseodymium promoted the incorporation of the iron ions in the defect fluorite structure. This effect has also been recently reported by Jovani et al. [6] using terbium as the lanthanide ion. Therefore, it seems that lanthanides promote the incorporation of a higher amount of iron in the lattice of $\mathrm{Y}_{2} \mathrm{Zr}_{2} \mathrm{O}_{7}$.

In order to study the oxidation states of the dopants, optical properties of the samples with single phase were investigated by UV/Vis spectroscopy and photoluminescence (PL). All powders exhibit a redorange shade colour with a different hue. Absorbance spectra of the fired powders are shown in Fig. $4 \mathrm{a}$ and $4 \mathrm{~b}$ in the range of 380-750 nm. The absorbance spectra of $\mathrm{Y}_{1.75} \mathrm{Pr}_{0.25} \mathrm{Zr}_{2} \mathrm{O}_{7}$ (labelled as P025) have been included for comparison in both spectra. A broad band is observed between 400 and $550 \mathrm{~nm}$ in all spectra, which could be associated to different $\mathrm{Fe}$ and $\operatorname{Pr}$ transitions [16,47,48]. Pr(III/IV) and Fe(II/III) transitions exist in the visible region of the spectrum but in these samples, the random distribution of the atoms in this lattice $(\mathrm{CN}=6.8-7.2)$ and the overlapping of the transitions does not enable a more detailed study to be undertaken.
The P025F025 and P035F025 presents the two highest absorptions in the range blue-violet region $(400-500 \mathrm{~nm})$, Fig. $4 \mathrm{a}$, when the iron content is fixed. This suggests that these powders present a greater redorange colouration and it could be related to the presence of a higher Pr (IV) concentration. It is known that the presence of $\operatorname{Pr}(\mathrm{IV})$ ions is associated with a wide absorption in this region $(330-590 \mathrm{~nm})$ due to the $4 \mathrm{f} \rightarrow 5 \mathrm{~d}$ transitions [49]. This fact suggests a higher amount of population of Pr(IV) in samples P025F025 and P035F025. Moreover, if this band is compared with those of P025 sample where $\operatorname{Pr}(\mathrm{III})$ is presented as the majority oxidation state (see explanation of the luminescence spectra in the next paragraph), it is clear that Pr(IV) plays an important role for the absorption intensity. On the other hand, if the praseodymium content is fixed, Fig. $4 \mathrm{~b}$, the iron plays also an important role, because it increases the intensity of the absorption bands in comparison with the sample without iron (P025).

In order to study the presence of the different oxidation states of $\mathrm{Pr}$, photoluminescence (PL) measurements were done to identify $\operatorname{Pr}(\mathrm{III})$ and they were compared with a sample with praseodymium as the only dopant (P025). Fig. 4c shows the emission spectra at room temperature for the samples that contain different amounts of praseodymium. These emission spectra were registered using an excitation wavelength of $308 \mathrm{~nm}$ in the range of $470-750 \mathrm{~nm}$. All samples exhibited one of the characteristic transitions of $\operatorname{Pr}(\mathrm{III})$ around $615 \mathrm{~nm},{ }^{1} \mathrm{D}_{2} \rightarrow{ }^{3} \mathrm{H}_{4}[25,50]$. Therefore, it confirmed the presence of $\operatorname{Pr}(\mathrm{III})$ in all samples. A clear trend can be observed from these spectra; when the amount of praseodymium increases, the intensity of PL also increases. It could be attributed to the effect of concentration in the samples. However, the difference of Pr(III) population is highlighted if P025 and P025F025 samples are compared. The amount of Pr(III) is higher in the first one, demonstrating that iron has an influence in this oxidation state of praseodymium, promoting a decrease of the population of $\operatorname{Pr}(\mathrm{III})$. Fig. $4 \mathrm{~d}$ also confirmed the oxidation of praseodymium when iron is present, and it does not depend on the iron concentration for these compositions.

This fact suggests that in certain way the 1 and 2 equations occur to a greater or lesser degree in all samples that contain the two dopants.

$\operatorname{Pr}^{3+} \rightarrow \operatorname{Pr}^{4+}+1 \mathrm{e}^{-}$

$\mathrm{Fe}^{3+}+1 \mathrm{e}^{-} \rightarrow \mathrm{Fe}^{2+}$

The oxidation of $\mathrm{Pr}^{3+}$ to $\mathrm{Pr}^{4+}$ involves the reduction of certain amount of $\mathrm{Fe}^{3+}$ to $\mathrm{Fe}^{2+}$. Therefore, all samples presented both oxidation states of each codopant, $\mathrm{Pr}^{3+} / \mathrm{Pr}^{4+}$ and $\mathrm{Fe}^{2+} / \mathrm{Fe}^{3+}$.

In order to confirm the mixed oxidation state of the iron dopant and relate it with the final colour of the powders, XPS was performed for all single-phase samples. The background due to inelastic scattered electrons was calculated for each reference spectrum, using the "smart" option present in the Avantage software. The $\mathrm{C} 1 \mathrm{~s}$ was used as an internal standard. Table 3 shows the values of the Fe2p photoelectron peaks and the distance between the photoelectron peak of $\mathrm{Fe} 2 \mathrm{p}_{3 / 2}$ and its satellite for all samples, and Fig. 5 shows the XPS spectra of Fe2p for P025F015 and P025F025 samples. The XPS peaks of Fe2 $\mathrm{p}_{3 / 2}$ and Fe2 $\mathrm{p}_{1}$, ${ }_{2}$ are observed. $\mathrm{Fe} 2 \mathrm{p}_{3 / 2}$ peak is narrower and stronger than $\mathrm{Fe} 2 \mathrm{p}_{1 / 2}$ peak because in spin-orbit ( $\mathrm{j}-\mathrm{j}$ ) coupling; $\mathrm{Fe} 2 \mathrm{p}_{3 / 2}$ has degeneracy of four states whilst Fe2p $\mathrm{p}_{1 / 2}$ has only two. The peak positions of $\mathrm{Fe} 2 \mathrm{p}_{3 / 2}$ has been investigated by many researchers and the values of between 710.6 and $711.2 \mathrm{eV}$ have been reported [51-54]. Energy separation between the $\mathrm{Fe} 2 \mathrm{p}_{3 / 2}$ and $\mathrm{Fe} 2 \mathrm{p}_{1 / 2}$ is also dependent on $\mathrm{Fe}$ ionic state since the character of the multiple splitting affects it. The presence or the absence of charge transfer satellite near the photoelectron peak also gives information about the oxidation state of iron. In a compound with Fe(II), satellite of $\mathrm{Fe} 2 \mathrm{p}_{3 / 2}$ constitutes a shoulder in the photoelectron peak, whereas for species with $\mathrm{Fe}(\mathrm{III})$, this same satellite is very distinctive from the photoelectron peak [55].

In our samples, Table 3 , the binding energies of the two $\mathrm{Fe} 2 \mathrm{p}_{3 / 2}$ and $\mathrm{Fe} 2 \mathrm{p}_{1 / 2}$ peaks are in the range of $710.4-710.7 \mathrm{eV}(\mathrm{S} . \mathrm{D} .=0.11)$ and 

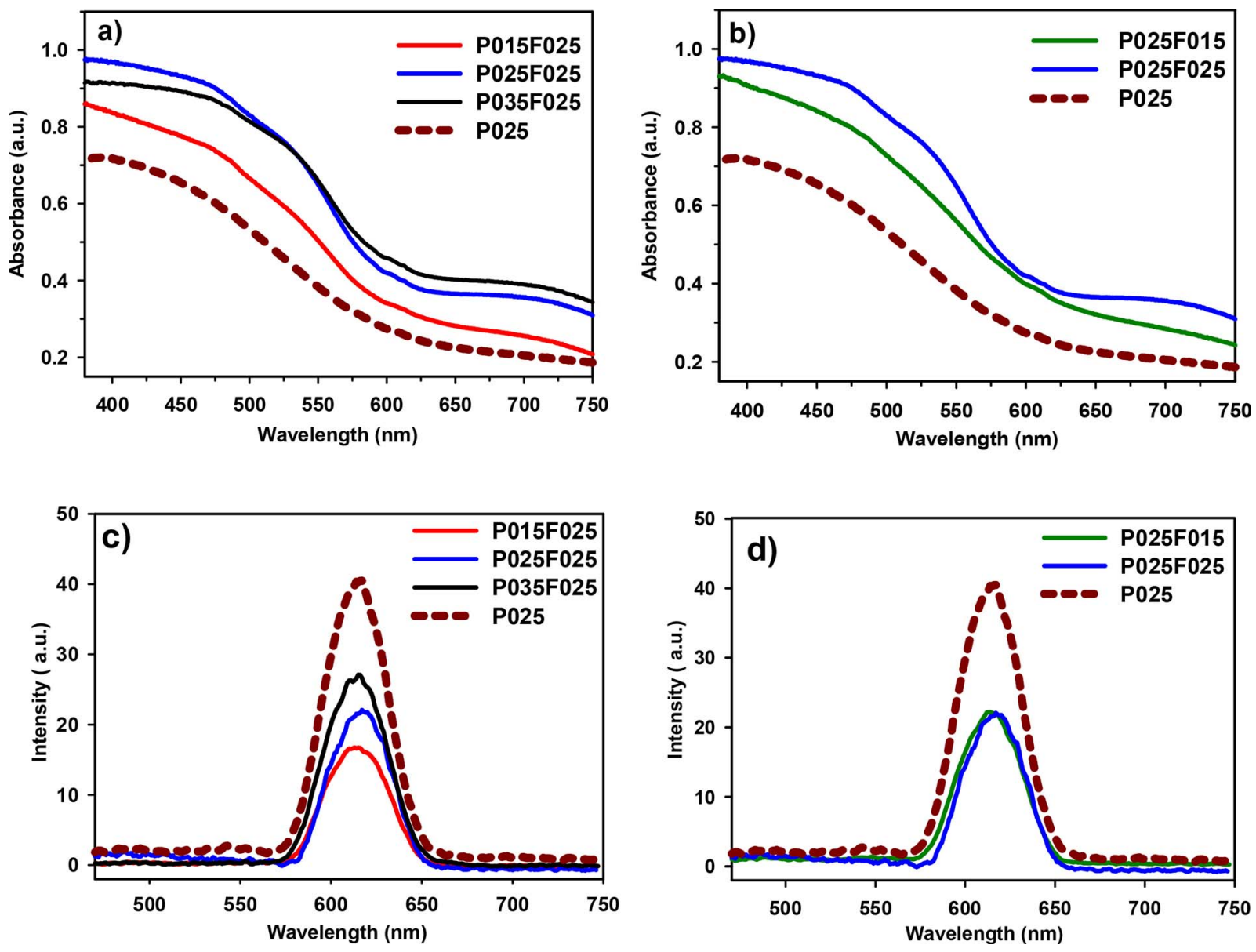

Fig. 4. UV-vis spectra (a and b) and photoluminescence spectra (c and d) of the powders fired at $1400{ }^{\circ} \mathrm{C}$.

Table 3

Peak positions of $\mathrm{Fe} 2 \mathrm{p}_{1 / 2}$ and $\mathrm{Fe} 2 \mathrm{p}_{3 / 2}$ for the different compositions.

\begin{tabular}{lllll}
\hline \multirow{2}{*}{ Sample } & \multicolumn{2}{l}{ Peak position $(\mathrm{eV})$} & \multirow{2}{*}{$\Delta \mathrm{E}\left(\right.$ Satellite-Fe2 $\mathrm{p}_{3 / 2}$ ) } \\
\cline { 2 - 4 } & $\mathrm{Fe} 2 \mathrm{p}_{1 / 2}$ & $\mathrm{Fe} 2 \mathrm{p}_{3 / 2}$ & Satellite & \\
\hline P015F025 & 724.4 & 710.6 & 715.7 & 5.1 \\
P025F025 & 724.2 & 710.4 & 716.1 & 5.7 \\
P035F025 & 724.4 & 710.6 & 716.7 & 6.1 \\
P025F015 & 723.7 & 710.7 & 717.1 & 6.4 \\
P025F025 & 724.2 & 710.4 & 716.1 & 5.7
\end{tabular}

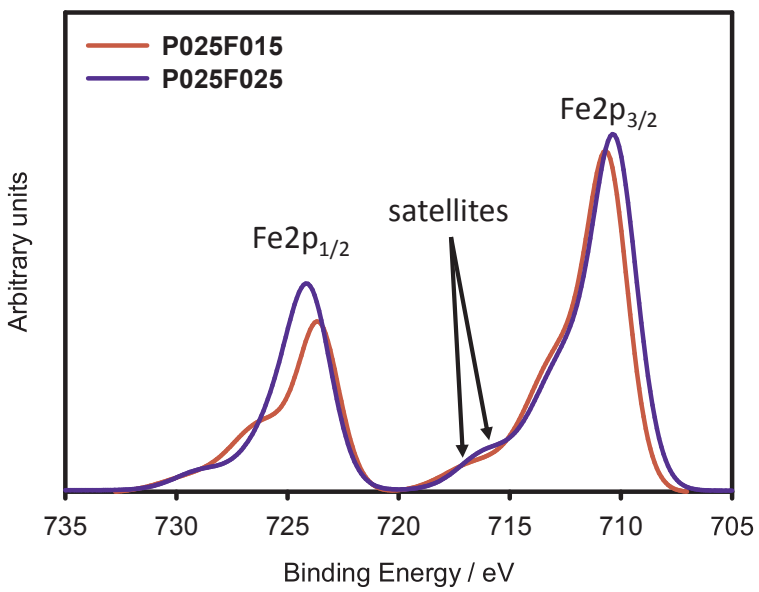

Fig. 5. XPS spectra for P025F015 and P025F025 samples.
723.7-724.4 eV (S.D. $=0.30$ ), respectively. The satellite peaks associated to $\mathrm{Fe}_{2} \mathrm{p}_{3 / 2}$ were observed in the range of $715.7-717.1 \mathrm{eV}$ $($ S.D. $=0.45)$. The binding energy difference between the Fe $2 \mathrm{p}_{3 / 2}$ peak and the satellite peak is approximately $6 \mathrm{eV}$. The presence of this 'shoulder' satellite peak and the binding energy difference are consistent with the results obtained by other authors [51-54,56], and is clear evidence of the existence of $\mathrm{Fe}(\mathrm{II})$ together with $\mathrm{Fe}(\mathrm{III})$ in all compositions studied.

Once the oxidation states of praseodymium and iron were identified, these could be related the final colour of the powders. The study of the colour is closely related with the oxidation state of the chromophores. Table 4 shows the chromatic coordinates of the powder fired at $1400{ }^{\circ} \mathrm{C}$. All samples present positive $\mathrm{a}^{*}$ and $\mathrm{b}^{*}$ values $\left(\mathrm{a}^{*} \gg>0\right.$ red colouration and $\mathrm{b}^{*} \gg>0$ yellow colouration) with good red-orange colouration, being P025F025 and P035F025 the two samples that present the best hue. These compositions have the highest value of $a^{*}$ and lowest of $\mathrm{b}^{*}$, and therefore, the best red-orange colouration.

Therefore, it seems clear that a suitable ratio of the oxidation states

Table 4

Chromatic coordinates of the powder samples and the powders in glass fired at $1080{ }^{\circ} \mathrm{C}$.

\begin{tabular}{|c|c|c|c|c|c|c|}
\hline & \multicolumn{3}{|c|}{ Powders } & \multicolumn{3}{|c|}{ Glass } \\
\hline & $\mathrm{L}^{*}$ & $a^{*}$ & $b^{*}$ & $\mathrm{~L}^{*}$ & $a^{*}$ & $b^{*}$ \\
\hline P015F025 & 50 & 13 & 28 & 55 & 18 & 28 \\
\hline P025F025 & 47 & 15 & 23 & 53 & 25 & 33 \\
\hline P035F025 & 45 & 14 & 21 & 51 & 22 & 29 \\
\hline P025F015 & 52 & 13 & 24 & 63 & 15 & 27 \\
\hline P025F025 & 47 & 15 & 23 & 53 & 25 & 33 \\
\hline
\end{tabular}




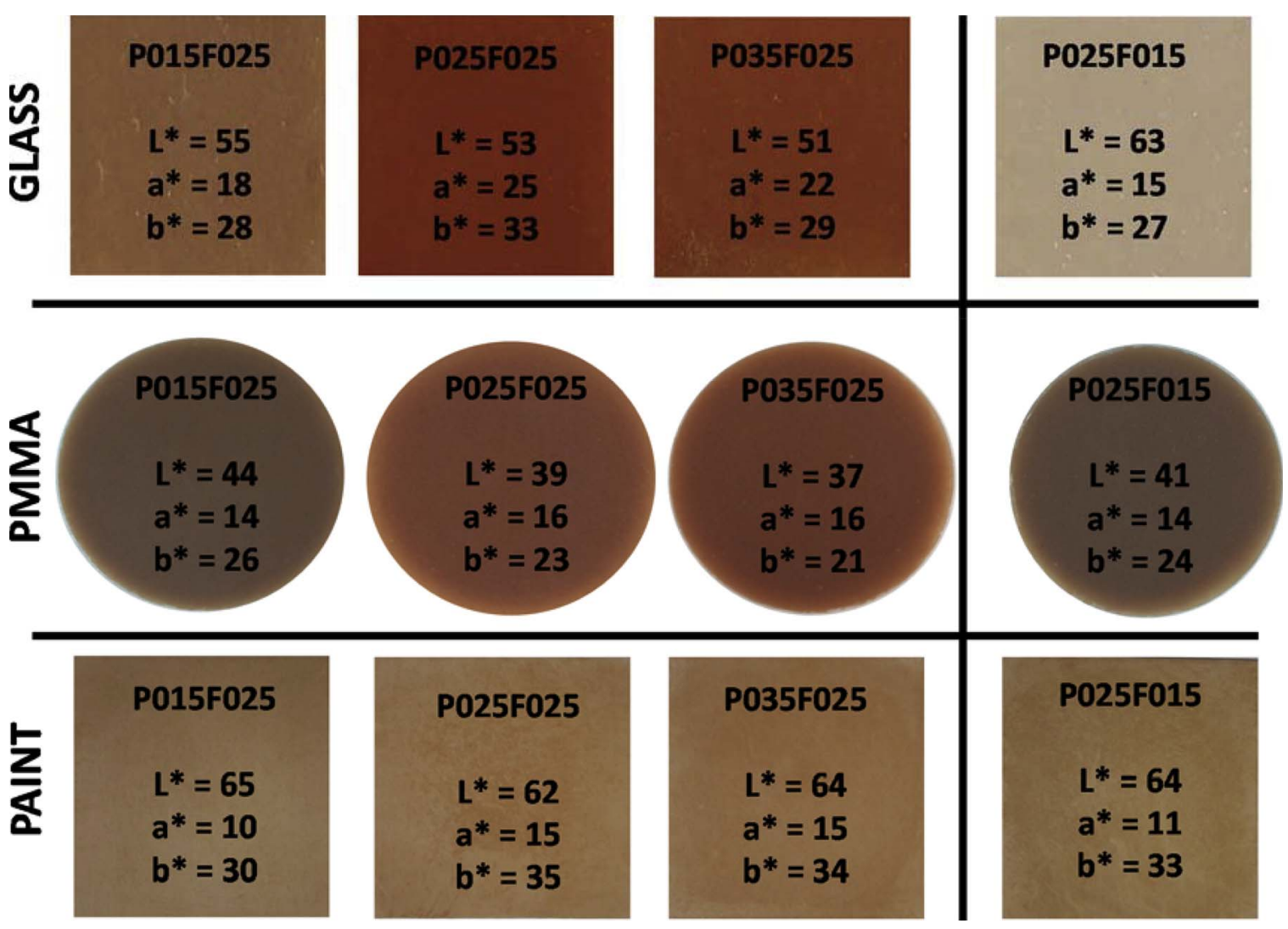

Fig. 6. Photographs and chromatic coordinates of the pigments mixed with glass, PMMA and siloxane paint.

of each codopant $\left(\mathrm{Pr}^{3+} / \mathrm{Pr}^{4+}\right.$ and $\left.\mathrm{Fe}^{2+} / \mathrm{Fe}^{3+}\right)$ is closely related with the colour final of the powder.

\subsection{Evaluation of the colouring performance of the pigment in glass}

The stability of the powders prepared at $1400{ }^{\circ} \mathrm{C}$ after glazing was tested. A powdered/frit mixture was prepared and fired according the cycle set out in the experimental part at the maximum temperature of $1080{ }^{\circ} \mathrm{C}$. The CIEL*a*b* parameters of the pieces with the glaze were obtained. A photograph of the compositions with a single phase after mixed with the frit, are shown in Fig. 6. Chromatic coordinates of the final coloured are also indicated in this figure. The pigment colour presented a better red colouration in glass than in powder for all samples. A significant increase of the $\mathrm{a}^{*}$ and $\mathrm{b}^{*}$ coordinates in the P025F025 and P035F025 samples was observed when they were mixed and fired in glass.

In order to understand the change in the colouration of the pigment in glass, PL of the mixture pigment/glass was studied for the P025F025 sample, which corresponds to that with the best chromatic coordinates. The results were compared with the PL of a mixture P025/glass. Fig. 7 shows the PL spectra of both samples. A change in the $\operatorname{Pr}(\mathrm{III}) / \operatorname{Pr}(\mathrm{IV})$ ratio is observed increasing the amount of $\operatorname{Pr}(\mathrm{IV})$ after glazing. Therefore, the glass promoted the oxidation of the praseodymium and as a consequence a possible change in the $\mathrm{Fe}(\mathrm{III}) / \mathrm{Fe}(\mathrm{II})$ ratio.

The importance of the $\mathrm{Pr}^{3+} / \mathrm{Pr}^{4+}$ and $\mathrm{Fe}^{2+} / \mathrm{Fe}^{3+}$ ratios have been reflected in the final colour of the pigments after introducing them in a glass. After this treatment, the oxidation states ratios were modified and the colours were changed. All samples present an optimal colouration, the P025F025 being the best composition. These results make this pigment a potential candidate for environmental red ceramic pigment.

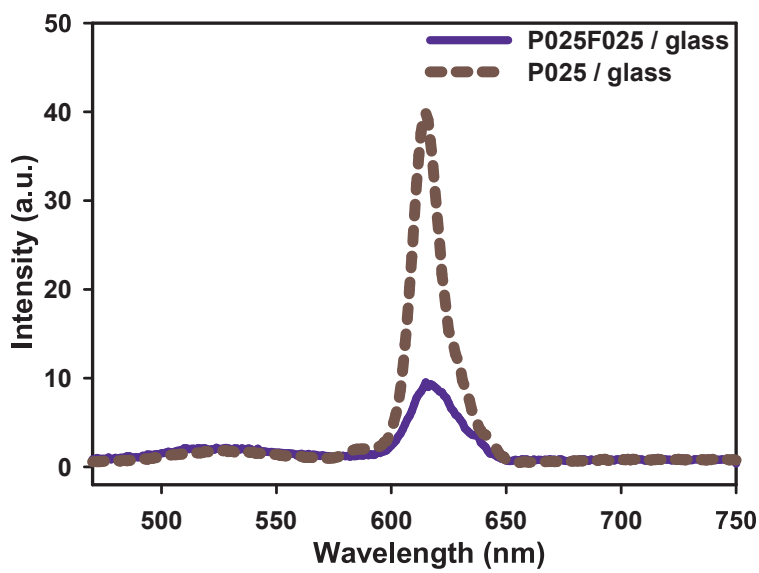

Fig. 7. Photoluminescence spectra for samples P025F025 and P025 mixed and fired in glass at the maximum temperature of $1080^{\circ} \mathrm{C}$

\subsection{Evaluation of the application of pigmented coatings and light resistance in plastic}

The colouring performance of the pigments was also investigated for its colouring application in a PMMA substrate. Typically, $10 \%$ of the pigment sample was dispersed in PMMA and compressed to a cylindrical disc. The photographs and the $\mathrm{L}^{*} \mathrm{a} * \mathrm{~b} *$ coordinates of the pieces are shown in Fig. 6. It becomes evident that the pigments promote colouration and opacity. In addition to that, the absence of deformities was verified, suggesting that the pigments were compatible with the polymer used. The colouration exhibited in PMMA was satisfactory, showing close chromatic coordinates to that in powder. Therefore, it suggests that there is no change in the oxidation state of the ions involved. 


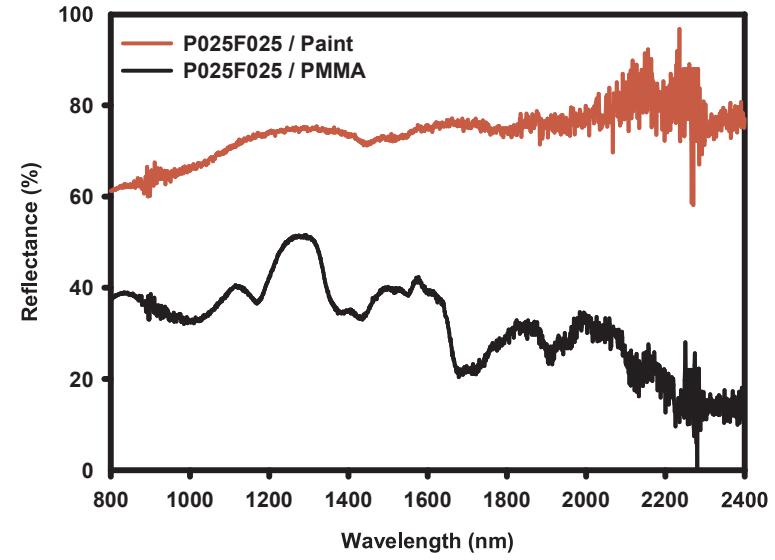

Fig. 8. NIR Reflectance spectra of P025F025 mixed with siloxane paint and PMMA.

The NIR solar pigmented PMMA was evaluated in Fig. 8. The solar reflectance of the pigmented PMMA acquired values around 50\% in reflectance, and suggested that this red-orange P025F025 pigment is a promising material for preparing building coatings with energy saving performance.

The light resistance of the typical pigmented polymer (10\%) was tested by exposing it to sunlight at various time intervals and measuring the colour coordinates. The $L^{*} \mathrm{a} * \mathrm{~b} *$ values after $6,12,24$ and $48 \mathrm{~h}$, were the same as that of the unexposed sample which indicates that the pigmented polymer is resistant to light. Thus, the developed pigments may find potential applications in colouring of various plastic materials.

\subsection{Evaluation of the thermal performance of the pigmented in paint}

Other possible applications of this pigment could be its use as "cool pigment" in paints. For this study, the samples were mixed with siloxane transparent paint. Siloxane is an impermeable paint providing a protection from the rainwater and ice increasing the durability of the surface where the paint is applied. Therefore, it seems very interesting to add to these features a high reflectance in order to obtain paint for exterior coatings. Fig. 6 shows the colour and CIEL*a*b* coordinates of each sample mixed with the paint and applied in a ceramic substrate. It shows that the P025F025 powder showed a good stability in the siloxane paint after applying it on the surface of the ceramic tile, decreasing slightly the initial colour of the powder.

In order to test the potential application of this pigment like an IR reflective material, spectral reflectance in the NIR region were done. Fig. 8 shows the NIR reflectance for the P025F025 sample in paint. The mixture paint/pigmented reached values around $80 \%$. This value is a higher than the majority of the commercially available IR reflective pigments [57]. Therefore, the optimal NIR reflectance of this pigment in paint, together with the use of environmental friendly elements, make this pigment optimal for these kinds of applications.

\section{Conclusions}

A new red-orange shade, environmentally friendly pigment based on the general formula $\mathrm{Y}_{2-\mathrm{x}} \mathrm{Pr}_{\mathrm{x}} \mathrm{Zr}_{2-\mathrm{y}} \mathrm{Fe}_{\mathrm{y}} \mathrm{O}_{7-\delta}$ was prepared by polymeric sol-gel method at $1400{ }^{\circ} \mathrm{C}$. Single phase of defect fluorite was obtained by XRD and SEM/EDX in all samples except in the composition with the highest amount of iron, therefore, the limit of the solid solution was $0.15 \leq \mathrm{x} \leq 0.35$ when $\mathrm{y}=0.25$ and $0 \leq \mathrm{y} \leq 0.25$ when $\mathrm{x}=0.25$. The oxidation states of both dopants were studied by UV/Vis absorption measurements, XPS and photoluminescence, and a possible redox mechanism was proposed. The oxidation of $\mathrm{Pr}^{3+}$ to $\mathrm{Pr}^{4+}$ involves the reduction of certain amount of $\mathrm{Fe}^{3+}$ to $\mathrm{Fe}^{2+}$. Therefore, all samples presented both oxidation states of each codopant. Praseodymium acts as a source of electrons for the reduction of iron, and this effect is increased when the pigment is incorporated in a glass.

The colour of the pigment was determined by CIEL*a*b* chromatic coordinates in powder, glass, paint and PMMA. All single-phase compositions presented a good stability and a good red-orange colouration in powder and in glass, the P025F025 being the composition with a best red good colouration in glass $\left(\mathrm{a}^{*} \gg>0\right.$ ). A suitable ratio of the oxidation states of each codopant $\left(\mathrm{Pr}^{3+} / \mathrm{Pr}^{4+}\right.$ and $\left.\mathrm{Fe}^{2+} / \mathrm{Fe}^{3+}\right)$ is close related with the final colour.

The pigments were also tested in siloxane paint. P025F025 also presents an optimal NIR reflectance acquiring values around $80 \%$ in paint. These results encourage this pigment to be used as cool colourants for roofing applications to mitigate the increase of the temperature inside buildings.

Finally, the performance of the pigments was studied for their application as colouring in a PMMA substrate. The CIEL*a*b* values obtained are very close to those of the powders, presenting a uniform distribution of the pigment particles in the polymer matrix. The typical total colour difference of chromatic coordinates after exposing to sunlight was negligible. This indicates that the pigmented polymer is light resistant.

A new red-orange shade multifunctional pigment, which does not contain toxic elements, was developed for its possible incorporation in different applications such as material in ceramic tiles, roofing materials and in plastics colouration.

\section{Acknowledgements}

M.J, M.F-M, H.B-M and E.C. thank the Universidad Jaume I (Project UJI-B2016-38) and Ministerio de Economia, Industria y Competitividad (Project MAT2016-80410-P) for financial support. The authors are grateful to Dr. Elena Serrano and Dr. Noemí Linares from Universidad de Alicante for the XPS analyses.

\section{References}

[1] T. Masui, T. Honda, N. Imanaka, Novel and environmentally friendly (Bi,Ca,Zn)VO yellow pigments, Dye Pigment. 99 (2013) 636-641.

[2] Wendusu, D. Kato, T. Masui, N. Imanaka, Novel environmentally friendly inorganic yellow pigments based on $\mathrm{CeO}_{2}-\mathrm{SiO}_{2}-\mathrm{Al}_{2} \mathrm{O}_{3}-\mathrm{Bi}_{2} \mathrm{O}_{3}$, Bull. Chem. Soc. Jpn. 86 (2013) 283-288.

[3] R.C. Olegário, E.C. Ferreira de Souza, J.F. Marcelino Borges, J.B. Marimon da Cunha, A.V. Chaves De Andrade, S.R. Masetto Antunes, A.C. Antunes, Synthesis and characterization of $\mathrm{Fe}^{3+}$ doped cerium-praseodymium oxide pigments, Dye. Pigment. 97 (2013) 113-117.

[4] B. Bae, S. Tamura, N. Imanaka, Novel environment-friendly yellow pigments based on praseodymium(III) tungstate, Ceram. Int. 43 (2017) 7366-7368.

[5] M. Martos, B. Julian-López, E. Cordoncillo, P. Escribano, Structural and spectroscopic study of a novel erbium titanate pink pigment prepared by sol-gel methodology, J. Phys. Chem. B. 112 (2008) 2319-2325.

[6] M. Jovaní, A. Sanz, H. Beltrán-Mir, E. Cordoncillo, New red-shade environmentalfriendly multifunctional pigment based on $\mathrm{Tb}$ and $\mathrm{Fe}$ doped $\mathrm{Y}_{2} \mathrm{Zr}_{2} \mathrm{O}_{7}$ for ceramic applications and cool roof coatings, Dye Pigment. 133 (2016) 33-40.

[7] V. De la Luz, M. Prades, H. Beltrán, E. Cordoncillo, Environmental-friendly yellow pigment based on $\mathrm{Tb}$ and $\mathrm{M}(\mathrm{M}=\mathrm{Ca}$ or $\mathrm{Ba})$ co-doped $\mathrm{Y}_{2} \mathrm{O}_{3}$, J. Eur. Ceram. Soc. 33 (2013) 3359-3368.

[8] L. Stránská, P. Šulcová, M. Vlček, Synthesis and properties of inorganic pigments based on pyrochlore compounds with different lanthanides, J. Therm. Anal. Calorim. 113 (2013) 127-135.

[9] R. Zallen, M.P. Moret, The optical absorption edge of brookite $\mathrm{TiO}_{2}$, Solid State Commun. 137 (2006) 154-157.

[10] M. Llusar, J.A. Badenes, J. Calbo, M.A. Tena, G. Monrós, Environmental and colour optimisation of mineraliser addition in synthesis of iron zircon ceramic pigment, $\mathrm{Br}$. Ceram. Trans. 99 (2000) 14-22.

[11] K. Pyon, B. Lee, Effect of iron content and annealing temperature on the color characteristics of $\mathrm{Fe}_{\mathrm{ZrSiO}}$ coral pink pigments synthesized by sol-gel method, J. Ceram. Soc. Jpn. 117 (2009) 258-263.

[12] G. Cappelletti, S. Ardizzone, P. Fermo, S. Gilardoni, The influence of iron content on the promotion of the zircon structure and the optical properties of pink coral pigments, J. Eur. Ceram. Soc. 25 (2005) 911-917.

[13] E. Carreto, C. Piña, H. Arriola, C. Barahona, A.N. Nava, V. Castaño, Mössbauer study of the structure of Fe - Zircon system, J. Radioanal. Nucl. Chem. 250 (2001) $453-458$.

[14] R.A. Eppler, Kinetics of Formation of an Iron-Zircon Pink Color, J. Am. Ceram. Soc. 62 (1979) 47-49.

[15] M. Llusar, J. Calbo, J.A. Badenes, M.A. Tena, G. Monrós, Synthesis of iron zircon 
coral by coprecipitation routes, J. Mater. Sci. 36 (2001) 153-163.

[16] M. Llusar, G. Monrós, C. Gargori, S. Cerro, R. Galindo, A. García, Iron and chromium doped perovskite $\left(\mathrm{CaMO}_{3} \mathrm{M}=\mathrm{Ti} \mathrm{Zr}\right)$ ceramic pigments, effect of mineralizer, Ceram. Int. 38 (2012) 4453-4460.

[17] T.E. Rodrigues Fiuza, J.F. Marcelino Borges, J.B. Marimon da Cucha, S.R. Masetto Antunes, A.V. Chaves de Andrade, A. Celso Antunes, E.C. Ferreira de Souza, Ironbased inorganic pigments from residue: preparation and application in ceramic, polymer, and paint, Dye. Pigment. 148 (2018) 319-328.

[18] E.A. Medina, J. Li, M.A. Subramanian, Colored oxides with hibonite structure II: Structural and optical properties of $\mathrm{CaAl}_{12} \mathrm{O}_{19}$-type pigments with chromophores based on Fe Mn, Cr and Cu, Prog. Solid State Chem. 45-46 (2017) 9-29.

[19] R.S. Pavlov, J.B. Carda Castelló, V. Blasco Marzá, J.M. Hohembergerger, New RedóShade ceramic pigments based on $\mathrm{Y}_{2} \mathrm{Sn}_{2-\mathrm{x}} \mathrm{Cr}_{\mathrm{x}} \mathrm{O}_{7-\delta}$ pyrochlore solid ssolutions, J. Am. Ceram. Soc. 85 (2002) 1197-1202.

[20] M.A. Subramanian, G. Aravamudan, G.V. Subba Rao, Oxide pyrochlores - a review, Prog. Solid State Chem. 15 (1983) 55-143.

[21] Z. Zhang, S.C. Middleburgh, M. de los Reyes, G.R. Lumpkin, B.J. Kennedy, P.E.R. Blanchard, E. Reynolds, Gradual structural evolution from pyrochlore to defect-Fluorite in $\mathrm{Y}_{2} \mathrm{Sn}_{2-\mathrm{x}} \mathrm{Zr}_{\mathrm{x}} \mathrm{O}_{7}$ : average vs local structure, J. Phys. Chem 117 (2013) 26740-26749.

[22] K. Asami, K. Kusakabe, N. Ashi, Y. Ohtsuka, Synthesis of ethane and ethylene from methane and carbon dioxide over praseodymium oxide catalysts, Appl. Catal. A 156 (1997) 43-56.

[23] B. Savoini, J.E. Muñoz Santiuste, R. González, Optical characterization of $\mathrm{Pr}^{3+}$ - doped yttria-stabilized zirconia single crystals, Phys. Rev. B. 56 (1997) 5856-5865.

[24] M. Fibrich, H. Jelínková, J. Šulc, K. Nejezchleb, V. Škoda, Pr: $\mathrm{YAlO}_{3}$ microchip laser, Opt. Lett. 35 (2010) 2556-2557.

[25] P. Boutinaud, R. Mahiou, E. Cavalli, M. Bettinelli, Red luminescence induced by intervalence charge transfer in $\operatorname{Pr}^{3+}$-doped compounds, J. Lumin. 122-123 (2007) 430-433.

[26] G. Del Nero, G. Cappelletti, S. Ardizzone, P. Fermo, S. Gilardoni, Yellow Pr-zircon pigments: the role of praseodymium and of the mineralizer, J. Eur. Ceram. Soc. 24 (2004) 3603-3611.

[27] L.S. Kumari, G. George, P.P. Rao, M.L.P. Reddy, The synthesis and characterization of environmentally benign praseodymium-doped $\mathrm{TiCeO}_{4}$ pigments, Dye. Pigment. 77 (2008) 427-431.

[28] J.H. Lee, Y.J. Kim, Luminescent properties of Pr doped $\mathrm{ZrSiO}_{4}$ phosphors, Ceram. Int. 34 (2008) 1113-1116.

[29] U. Kempe, S.M. Thomas, G. Geipel, R. Thomas, M. Plötze, R. Böttcher, G. Grambole, J. Hoentsch, M. Trinkler, Optical absorption, iuminescence, and electron paramagnetic resonance (EPR) spectroscopy of crystalline to metamict zircon: evidence for formation of uranyl, manganese, and other optically active centers, Am. Mineral. 95 (2010) 335-347.

[30] D.J. Taylor, P.F. Fleig, R.A. Page, Characterization of nickel titanate synthesized by sol-gel processing, Thin Solid Films 408 (2002) 104-110.

[31] J. Zou, P. Zhang, C. Liu, Y. Peng, Highly dispersed (Cr, Sb)-co-doped rutile pigments of cool color with high near-infrared reflectance, Dye. Pigment. 109 (2014) 113-119.

[32] R. Levinson, P. Berdahl, H. Akbari, Solar spectral optical properties of pigments - Part II: survey of common colorants, Sol. Energy Mater. Sol. Cells 89 (2005) 351-389.

[33] V. Fang, J. Futter, J. Kennedy, J. Manning, A Review of near Infrared Reflectance Properties of Metal Oxide Nanostructures GNS Science Report, (2013).

[34] S. Radhika, K.J. Sreeram, B.U. Nair, Effective synthesis route for red-brown pigments based on $\mathrm{Ce}-\mathrm{Pr}-\mathrm{Fe}-\mathrm{O}$ and their potential application for near infrared reflective surface coating, J. Chem. Sci. 126 (2014) 65-73.

[35] Z. Tao, W. Zhang, Y. Huang, D. Wei, H.J. Seo, A novel pyrophosphate $\mathrm{BaCr}_{2}\left(\mathrm{P}_{2} \mathrm{O}_{7}\right)_{2}$ as green pigment with high NIR solar reflectance and durable chemical stability, Solid State Sci. 34 (2014) 78-84.

[36] R. Levinson, P. Berdahl, H. Akbari, W. Miller, I. Joedicke, J. Reilly, Y. Suzuki, M. Vondran, Methods of creating solar-reflective nonwhite surfaces and their application to residential roofing materials, Sol. Energy Mater. Sol. Cells 91 (2007)
304-314

[37] A. Libbra, L. Tarozzi, A. Muscio, M.A. Corticelli, Spectral response data for development of cool coloured tile coverings, Opt. Laser Technol. 43 (2011) 394-400.

[38] A. Han, M. Zhao, M. Ye, J. Liao, Z. Zhang, N. Li, Crystal structure and optical properties of $\mathrm{YMnO}_{3}$ compound with high near-infrared reflectance, Sol. Energy 91 (2013) 32-36.

[39] L.S. Kumari, G. George, P.P. Rao, M.L.P. Reddy, The synthesis and characterization of environmentally benign praseodymium-doped $\mathrm{TiCeO}_{4}$ pigments, Dye. Pigment. 77 (2008) 427-431.

[40] F.G. Maranha, T.E. Rodrigues Fiuza, E.C. Ferreira de Souza, J.F. Marcelino Borges, J.B. Marimon da Cunha, A.V. Chaves de Andrade, S.R. Masetto Antunes, A.C. Antunes, Synthesis and characterization of pigments of the $\mathrm{LaAl}_{1-\mathrm{x}} \mathrm{Fe}_{\mathrm{x}} \mathrm{O}_{3}$ system ? Application in ceramic and polymer, Dye Pigment. 133 (2016) 304-310.

[41] V. James, P. Prabhakar Rao, S. Sameera, S. Divya, Multiferroic based reddish brown pigments: $\mathrm{bi}_{1-\mathrm{x}} \mathrm{M}_{\mathrm{x}} \mathrm{FeO}_{3}(\mathrm{M}=\mathrm{Y}$ and La) for coloring applications, Ceram. Int. 40 (2014) 2229-2235.

[42] G. George, L. Sandhya Kumari, V.S. Vishnu, S. Ananthakumar, M.L.P. Reddy, Synthesis and characterization of environmentally benign calcium-doped $\mathrm{Pr}_{2} \mathrm{Mo}_{2} \mathrm{O}_{9}$ pigments: applications in coloring of plastics, J. Solid State Chem. 181 (2008) 487-492.

[43] S. Soumya, A.P. Mohamed, K. Mohan, S. Ananthakumar, Enhanced near-infrared reflectance and functional characteristics of Al-doped $\mathrm{ZnO}$ nano-pigments embedded PMMA coatings, Sol. Energy Mater. Sol. Cells 143 (2015) 335-346.

[44] V.S. Vishnu, G. George, V. Divya, M.L.P. Reddy, Synthesis and characterization of new environmentally benign tantalum-doped $\mathrm{Ce}_{0.8} \mathrm{Zr}_{0.2} \mathrm{O}_{2}$ yellow pigments: applications in coloring of plastics, Dye. Pigment. 82 (2009) 53-57.

[45] R.M. Harris, Coloring Technology for Plastics, Plastics Design Library, New York, 1999.

[46] R.D. Shannon, Revised effective ionic radii and systematic studies of interatomic distances in halides and chalcogenides, Acta Cryst. A32 (1976) 751-767.

[47] L.S. Kumari, P.P. Rao, P. Koshy, Red pigments based on $\mathrm{CeO}_{2}-\mathrm{MO}_{2}-\mathrm{Pr}_{6} \mathrm{O}_{11}(\mathrm{M}=\mathrm{Zr}$ and Sn): Solid solutions for the coloration of plastics, J. Am. Ceram. Soc. 93 (2010) 1402-1408.

[48] M. Dondi, F. Matteucci, G. Cruciani, G. Gasparotto, D.M. Tobaldi, Pseudobrookite ceramic pigments: crystal structural, optical and technological properties, Solid State Sci. 9 (2007) 362-369.

[49] N. Montoya, G. Herrera, J. Alarco, Synthesis and characterization of praseodymium containing $\mathrm{ZrSiO}_{4}$ solid solutions from gels, Ceram. Int. 37 (2011) 3609-3616.

[50] E. Pinel, P. Boutinaud, R. Mahiou, Using a structural criterion for the selection of red-emitting oxide-based compounds containing $\mathrm{Pr}^{3+}$, J. Alloys Compd. 374 (2004) $165-168$.

[51] S.J. Roosendaal, B. Van Asselen, J.W. Elsenaar, A.M. Vredenberg, F.H.P.M. Habraken, The oxidation state of $\mathrm{Fe}(100)$ after initial oxidation in $\mathrm{O}_{2}$, Surf. Sci. 442 (1999) 329-337.

[52] P.C.J. Graat, M.A.J. Somers, Simultaneous determination of composition and thickness of thin iron-oxide films from XPS Fe2p spectra, Appl. Surf. Sci. 100-101 (1996) 36-40.

[53] P. Mills, J.L. Sullivan, A study of the core level electrons in iron and its three oxides by means of X-ray photoelectron spectroscopy, J. Phys. D Appl. Phys. 16 (1983) $723-732$.

[54] M. Muhler, R. Schlögl, G. Ertl, The nature of the iron oxide-based catalyst for dehydrogenation of ethylbenzene to styrene 2 . Surface chemistry of the active phase, J. Catal 138 (1992) 413-444.

[55] M. Descostes, F. Mercier, N. Thromat, C. Beaucaire, M. Gautier-soyer, Use of XPS in the determination of chemical environment and oxidation state of iron and sulfur samples: constitution of a data basis in binding energies for Fe and S reference compounds and applications to the evidence of surface species of an oxidized pyrite in a carbonate medium, Appl. Surf. Sci. 165 (2000) 288-302.

[56] T. Yamashita, P. Hayes, Analysis of XPS spectra of $\mathrm{Fe}^{2+}$ and $\mathrm{Fe}^{3+}$ ions in oxide materials, Appl. Surf. Sci. 254 (2008) 2441-2449.

[57] A.K. Bendiganavale, V.C. Malshe, Infrared reflective inorganic pigments, Recent Patents Chem. Eng. 1 (2008) 67-79. 


\subsection{Discusión de los resultados}

En el presente estudio se ha conseguido sintetizar disoluciones sólidas de la composición $\mathrm{Y}_{2} \mathrm{Zr}_{2} \mathrm{O}_{7}$ dopados con Pr y Fe a través del método sol-gel polimérico calcinadas a $1400^{\circ} \mathrm{C}$ durante $12 \mathrm{~h}$. Se realizaron dos grupos diferentes de composiciones basados la formula general $\mathrm{Y}_{2-\mathrm{x}} \mathrm{Pr}_{\mathrm{x}} \mathrm{Zr}_{2-\mathrm{y}} \mathrm{Fe}_{\mathrm{y}} \mathrm{O}_{7-\delta}$. Un primer grupo donde se fijó la cantidad de praseodimio en un valor de $\mathrm{x}=0.25$ y se varió la cantidad de hierro y un segundo grupo donde se fijó la cantidad de hierro y $=0.25$ y se varió la de praseodimio. Las composiciones y las referencias de las muestras sintetizadas se muestran en la tabla 4.

Tabla 4. Composiciones preparadas basadas en $\mathrm{Y}_{2-\mathrm{x}} \mathrm{Pr}_{\mathrm{x}} \mathrm{Zr}_{2-\mathrm{y}} \mathrm{Fe}_{\mathrm{y}} \mathrm{O}_{7-\delta}$.

\section{Referencia Composición}

\begin{tabular}{l|l}
\hline \hline P025F015 & $\mathrm{Y}_{1.75} \mathrm{Pr}_{0.25} \mathrm{Zr}_{1.85} \mathrm{Fe}_{0.15} \mathrm{O}_{6.925}$ \\
P025F035 & $\mathrm{Y}_{1.75} \mathrm{Pr}_{0.25} \mathrm{Zr}_{1.65} \mathrm{Fe}_{0.35} \mathrm{O}_{6.825}$ \\
\hline P015F025 & $\mathrm{Y}_{1.85} \mathrm{Pr}_{0.15} \mathrm{Zr}_{1.75} \mathrm{Fe}_{0.25} \mathrm{O}_{6.875}$ \\
P025F025 & $\mathrm{Y}_{1.75} \mathrm{Pr}_{0.25} \mathrm{Zr}_{1.75} \mathrm{Fe}_{0.25} \mathrm{O}_{6.875}$ \\
P035F025 & $\mathrm{Y}_{1.65} \mathrm{Pr}_{0.35} \mathrm{Zr}_{1.75} \mathrm{Fe}_{0.25} \mathrm{O}_{6.875}$
\end{tabular}

Se obtuvieron disoluciones sólidas con una estructura de fluorita defectuosa en todas las muestras excepto en la muestra con mayor cantidad de hierro (P025F035), la que presentó una fase secundaria atribuible a una estructura perovsquita $\mathrm{YFeO}_{3}$, detectada a partir de por difracción de rayos X (DRX). El análisis microestructural mediante, microscopía electrónica de barrido (SEM) y microanálisis por energías dispersivas de rayos X (EDX), confirmó los resultados obtenidos por DRX. 
Con el objetivo de estudiar los estados de oxidación y su influencia en el color, también ser realizarón medidas espectroscópicas de UV/Vis, fotolumiscencia (PL) y espectrometría fotoelectrónica de rayos X (XPS), de las muestras que presentaron fase única de fluorita defectuosa. La espectroscopia UV/Vis mostró una banda ancha en la zona de 400-550 nm asociada a las transiciones de ambos cromóforos. Sin embargo, las muestras con mayor cantidad de hierro y praseodimio (P025F025 y P035F025) mostraron las mayores absorciones en la zona azul-violeta del espectro (400-500 nm), asociadas a las transiciones $4 f \rightarrow 5 d$ del $\operatorname{Pr}^{4+}$. Esta mayor absorción en esta región provocara una mejor coloración roja-naranja, por lo que nos sugiere que el $\mathrm{Pr}^{4+}$ juega un papel fundamental en el desarrollo del color final. Por este motivo se realizaron medidas de fotolumiscencia para poder determinar la presencia de iones $\operatorname{Pr}^{3+}$ y su variación a diferentes cantidades de hierro. Los espectros de emisión de las muestras excitadas a $308 \mathrm{~nm}$ pusieron de manifiesto al comparar muestras con la misma cantidad de praseodimio con y sin hierro, la presencia de este último provoca una disminución en la población de iones $\operatorname{Pr}^{+3}$ provocando por tanto la oxidación de $\operatorname{Pr}^{3+}$ a $\operatorname{Pr}^{4+}$.

A partir de estos resultados y respaldados con los resultados de absorción se formuló un hipotético mecanismo, como muestra la ecuación 14, explicando cómo afecta la presencia del hierro al estado de oxidación del praseodimio.

$$
\begin{gathered}
\mathrm{Pr}^{3+} \rightarrow \mathrm{Pr}^{4+}+1 \mathrm{e}^{-} \\
\mathrm{Fe}^{3+}+1 \mathrm{e}^{-} \rightarrow \mathrm{Fe}^{2+}
\end{gathered}
$$

Por lo tanto, parece que la presencia de hierro promueve la oxidación del praseodimio, siendo las muestras con más cantidad de hierro las que son capaces de oxidar mayor cantidad de praseodimio. Estos resultados se confirmaron con 
medidas de XPS, en los que se detectó en todas las muestras ambos estados de oxidación en los dos elementos cromóforos $\mathrm{Pr}^{3+} / \mathrm{Pr}^{4+}$ y Fe ${ }^{2+} / \mathrm{Fe}^{3+}$.

Como se ha mencionado en la introducción del presente capítulo, los estados de oxidación están fuertemente ligados al desarrollo de la coloración final de los polvos sintetizados. Las coordenadas cromáticas mostraron que en las $\begin{array}{lllll}\text { muestras } & \text { P025F025 } & \left(\mathrm{Y}_{1.75} \mathrm{Pr}_{0.25} \mathrm{Zr}_{1.75} \mathrm{Fe}_{0.25} \mathrm{O}_{6.875}\right) & \text { y } & \text { P035F025 }\end{array}$ ( $\mathrm{Y}_{1.65} \mathrm{Pr}_{0.35} \mathrm{Zr}_{1.75} \mathrm{Fe}_{0.25} \mathrm{O}_{6.875}$ ) se obtuvieron los mayores valores de $\mathrm{a}^{*}$ (a*>0 rojo) y menores de coordenada $b^{*}$ (b*>0 amarilla), mostrando las mejores coloraciones rojizas. Parece claro que en esta estructura la cantidad de iones $\operatorname{Pr}^{4+}$ y de $\mathrm{Fe}^{3+}$ juegan un papel fundamental para la obtención de una coloración rojiza óptima.

Con el principal objetivo de comprobar el potencial uso de las disoluciones sintetizadas en diferentes tipos de aplicaciones, se mezclaron los pigmentos en vidriado, pintura y polimetilmetacrilato (PMMA).

Todas las composiciones presentaron una excelente estabilidad en el vidriado, exhibiendo una mejor coloración rojiza que en polvo. Este incremento de la coloración se puede explicar por un aumento de la cantidad de iones $\operatorname{Pr}^{4+}$ en el esmalte, como se confirmó realizando medidas de fotoluminiscencia también en el esmalte en la muestra P025F025.

Esta misma estabilidad también se puso de manifiesto en el PMMA, manteniéndose las mismas coordenadas cromáticas que en la muestra en polvo, por lo que se puede afirmar que no hay cambios de oxidación de los cromóforos, como sí que ocurre en el esmalte. Se realizaron medidas de reflectancia en la zona de NIR, para la muestra P025F025, para comprobar su potencial aplicabilidad en la industria de los polímeros como "cool pigment". Se obtuvieron valores muy satisfactorios de alrededor del 50\% de reflectancia. Además, también se estudió la estabilidad del pigmento en el polímero a la exposición solar, no produciéndose ninguna disminución en las coordenadas cromáticas. 
Adicionalmente, también se mezcló esta misma composición con pintura transparente de siloxano y se midió su reflectancia para comprobar su potencial aplicación. Se obtuvieron valores muy satisfactorios de alrededor del 80\%. Por lo tanto, parece que esta composición es óptima para su potencial aplicación como "cool pigment" en pinturas.

Por consiguiente, se sintetizó un nuevo pigmento cerámico de coloración rojiza-anaranjada sin el uso de elementos tóxicos como cromóforos. Se estableció el límite de la disolución sólida y se estudiaron los estados de oxidación y su influencia en el color final. Además, se comprobó su potencial uso en diferentes aplicaciones tales como esmalte cerámico, pintura y polímero, obteniéndose resultados altamente satisfactorios. 


\subsection{Conclusiones del artículo científico 2}

- Se han optimizado los parámetros de síntesis de la ruta sol-gel polimérico y del posterior tratamiento térmico para la formación de disoluciones sólidas basadas en la estequiometría $\mathrm{Y}_{2} \mathrm{Zr}_{2} \mathrm{O}_{7}$, dopadas con hierro y praseodimio.

- Se han determinado los límites de las disoluciones sólidas para la composición $\mathrm{Y}_{2-\mathrm{x}} \mathrm{Pr}_{\mathrm{x}} \mathrm{Zr}_{2-\mathrm{y}} \mathrm{Fe}_{\mathrm{y}} \mathrm{O}_{7-\delta}$, adquiriendo valores de $0.15 \leq \mathrm{x} \leq 0.35$ cuando $\mathrm{y}=0.25$, $\mathrm{y}$ $0 \leq \mathrm{y} \leq 0.25$ cuando $\mathrm{x}=0.25$. Presentando todas las composiciones en estos intervalos una estructura de fluorita defectuosa.

- Se han determinado los estados de oxidación de ambos cromóforos, en todas las disoluciones sólidas, presentando estos ambos estados de oxidación $\operatorname{Pr}^{3+} / \operatorname{Pr}^{4+} \mathrm{y}$ $\mathrm{Fe}^{2+} / \mathrm{Fe}^{3+}$.

- Se ha formulado un mecanismo para explicar los estados de oxidación de los cromóforos y cómo influye este en la coloración final. Se concluyó que la mejor coloración viene dada cuando más grandes son las cantidades de iones $\mathrm{Fe}^{3+}$ y de $\operatorname{Pr}^{4+}$.

- Se ha determinado el papel fundamental del ion $\mathrm{Pr}^{3+}$ actuando como fuente de electrones, oxidándose a $\operatorname{Pr}^{4+}$ y reduciendo cierta cantidad del hierro a iones $\mathrm{Fe}^{+2}$. 
- Se ha comprobado la estabilidad de las disoluciones sólidas en diferentes medios tales como esmalte, pintura y polímero. Las muestras mostraron una excelente estabilidad tanto en esmalte como en el polímero.

- La disolución sólida $\mathrm{Y}_{1.75} \mathrm{Pr}_{0.25} \mathrm{Zr}_{1.75} \mathrm{Fe}_{0.25} \mathrm{O}_{6.875}$ (P025F025) presentó la mejor coloración rojiza, tanto en polvo como en esmalte.

- Se ha comprobado el potencial uso de los pigmentos sintetizados en el campo de los "cool pigment". Para ello se realizaron medidas de reflectancia de las disoluciones sólidas en pintura y en polímero. La muestra P025F025 presentó unos valores óptimos de reflectancia en la zona del NIR de alrededor del 50\% en polímero y del $80 \%$ en pintura.

Se ha sintetizado un pigmento de coloración rojiza-naranja, sin el uso de elementos tóxicos, con una buena coloración en esmalte y en PMMA. Además, presenta propiedades multifuncionales, ya que presenta unas propiedades óptimas en el campo de los denominados "cool pigments" con una reflectancia en el infrarrojo cercano de alrededor del 80\% en pintura y 50\% en PMMA. 


\section{CAPítulO 5}

Conducción electrónica en conductores de ion óxido basados en $\mathrm{Zr}_{1-\mathrm{x}} \mathrm{Y}_{\mathrm{x}} \mathrm{O}_{2-\mathrm{x} / 2 \text {. }}$.

Dependencia de la atmosfera y del voltaje aplicado

"En algún lugar, alguna cosa increíble aguarda a ser descubierta" 



\subsection{Introducción}

En este capítulo se van a estudiar las propiedades eléctricas de diferentes disoluciones sólidas del sistema $\mathrm{ZrO}_{2}-\mathrm{Y}_{2} \mathrm{O}_{3}$, con cantidades mayores de dopante al $8 \%$ molar en itria. Para ello se sintetizaron diferentes composiciones con diferentes relaciones molares de $\mathrm{ZrO}_{2} / \mathrm{Y}_{2} \mathrm{O}_{3}$, mediante el método sol-gel polimérico y posteriormente se calcinaron a $1300^{\circ} \mathrm{C}$ durante $12 \mathrm{~h}$. Este método de síntesis proporciona una mayor reactivad que el método cerámico tradicional, permitiendo adicionalmente la posibilidad de estabilizar fases mestaestables y con una pureza muy elevada. El uso del mismo supone una ventaja a la hora de sintetizar composiciones, las cuales según los diagramas de fases conocidos no pueden ser estables a presiones y temperaturas ordinarias. Por lo tanto, a la temperatura de $1300^{\circ} \mathrm{C}$, según el diagrama de fases propuesto por Chen y col.[34], la máxima cantidad de $\mathrm{YO}_{1.5}$ que se puede introducir en la estructura de $\mathrm{ZrO}_{2}$ estabilizando la fase cúbica, es de alrededor de una fracción molar de 0.47 de $\mathrm{YO}_{1.5}$. A partir de esta composición y hasta alrededor de una fracción molar de $\mathrm{YO}_{1.5}$ de 0.57 , se obtendrán mezclas de la fase cúbica dopada con itrio y la fase romboédrica de estequiometría $\mathrm{Zr}_{3} \mathrm{Y}_{4} \mathrm{O}_{12}$. A fracciones molares superiores de 0.57 se estabiliza la fase romboédrica.

Posiblemente debido a la dificultad presentada para sintetizar estequiometrías ricas en $\mathrm{Y}_{2} \mathrm{O}_{3}$ por el método cerámico, son muy escasos los estudios de propiedades eléctricas a concentraciones superiores de itria al 8\% molar. Sin embargo, las propiedades eléctricas de la estructura $\mathrm{Y}_{2} \mathrm{Zr}_{2} \mathrm{O}_{7}$, es decir 50-50 \% molar de $\mathrm{ZrO}_{2}-\mathrm{YO}_{1.5}$, ha sido estudiada por dos autores hasta la época $[63,180,181]$. 
Kumar y col. han realizado dos estudios sobre la conductividad de la estructura $\mathrm{Y}_{2} \mathrm{Zr}_{2} \mathrm{O}_{7}$ dopada con bismuto y manganeso [63,180]. En ellos se determinó la conductividad del bulk, alcanzando valores de $3.75 \cdot 10^{-3} \mathrm{~S} \cdot \mathrm{cm}^{-1}$ a $800^{\circ} \mathrm{C}$. Claramente la conductividad exhibida es inferior a la presentada por el YSZ08 de $0.025 \mathrm{~S} \cdot \mathrm{cm}^{-1}$ a la misma temperatura [182].

Por otra parte, Gilardi y col han realizado estudios de las propiedades eléctricas de $\mathrm{Y}_{2} \mathrm{Zr}_{2} \mathrm{O}_{7}$ depositados vía PLD (Deposición Pulsada por Laser ) sobre substratos de óxido de magnesio [181].

En ningún caso, se han realizado estudios de conductividad a diferentes presiones parciales de oxígeno, ni se ha estudiado la influencia de la aplicación de un voltaje para esta estequiometría ni para otras estequiometrías ricas en itrio.

Por lo tanto, conociendo los antecedentes de aplicación de voltaje en electrocerámicas dopadas con iones aceptores, así como en el YSZ08 y conociendo la posible influencia en las propiedades eléctricas de las cantidades de dopante aceptor presentadas por el material, en este capítulo se estudiarán las propiedades eléctricas en diferentes composiciones del sistema $\mathrm{ZrO}_{2}-\mathrm{Y}_{2} \mathrm{O}_{3}$.

Para ello se sintetizaron composiciones $\mathrm{Zr}_{1-\mathrm{x}} \mathrm{Y}_{\mathrm{x}} \mathrm{O}_{2-\mathrm{x} / 2}(\mathrm{x}>0.4)$ mediante el método sol-gel polimérico y se estudiaron sus propiedades eléctricas, realizando medidas de impedancia a diferentes presiones parciales de oxígeno, así como con la aplicación de pequeños voltajes.

Este estudio dio lugar a una publicación en la revista internacional Inorganic Chemistry. En el siguiente punto se van a exponer los objetivos principales del presente capítulo. 


\subsection{Objetivos del artículo científico 3}

Los objetivos generales del presente trabajo son:

- Sintetizar mediante el método sol-gel polimérico disoluciones sólidas con

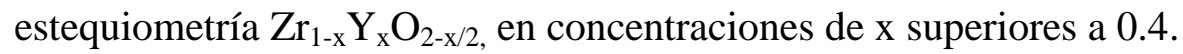

- Estabilizar la fase cúbica en la circona de estequiometría $\mathrm{Zr}_{1-\mathrm{x}} \mathrm{Y}_{\mathrm{x}} \mathrm{O}_{2-\mathrm{x} / 2}$ en concentraciones de itrio superiores 0.4 a $1300^{\circ} \mathrm{C}$.

- Determinar el límite de la disolución sólida en la estequiometría $\mathrm{Zr}_{1-\mathrm{x}} \mathrm{Y}_{\mathrm{x}} \mathrm{O}_{2-\mathrm{x} / 2}$.

- Estudiar las propiedades eléctricas de disoluciones sólidas preparadas con altas concentraciones de itrio mediante espectroscopia de impedancia compleja (EI).

- Estudiar los mecanismos de conducción de las diferentes disoluciones sólidas a diferentes presiones parciales de oxígeno.

- Estudiar el efecto de la aplicación de pequeños voltajes $d c$ en las composiciones sintetizadas y la influencia en sus propiedades eléctricas.

- Estudiar las propiedades eléctricas resultantes de la variación de las cantidades de ion estabilizador, para entender su influencia en las propiedades eléctricas. 


\subsection{Artículo científico 3}

Título: Atmosphere- and Voltage-Dependent Electronic Conductivity of Oxide-Ion-Conducting $\mathrm{Zr}_{1-\mathrm{x}} \mathrm{Y}_{\mathrm{x}} \mathrm{O}_{2-\mathrm{x} / 2}$

Revista: Inorganic Chemistry

Autores: Marc Jovaní, Héctor Beltrán-Mir, Eloisa Cordoncillo, Anthony R. West

Índice de Impacto: 4.7

Revista dentro del $25 \%$ : $\mathrm{Si}$

Categoría: Química inorgánica

Posición: 4/46 


\title{
Atmosphere- and Voltage-Dependent Electronic Conductivity of Oxide-lon-Conducting $\mathrm{Zr}_{1-x} \mathrm{Y}_{x} \mathrm{O}_{2-x / 2}$ Ceramics
}

\author{
Marc Jovaní, ${ }^{\dagger}$ Héctor Beltrán-Mir, ${ }^{\dagger}{ }^{\dagger}$ Eloisa Cordoncillo, ${ }^{\dagger}$ and Anthony R. West ${ }^{\ddagger}$ \\ ${ }^{\dagger}$ Departamento de Química Inorgánica y Orgánica, Universidad Jaume I, Avenida Sos Baynat s/n, Castellón 12071, Spain \\ ${ }^{\ddagger}$ Department of Materials Science \& Engineering, University of Sheffield, Mappin Street, Sheffield S1 3JD, U.K.
}

ABSTRACT: Cubic, fluorite-structured solid solutions $\mathrm{Zr}_{1-x} \mathrm{Y}_{x} \mathrm{O}_{2-x / 2}$ (YSZ; $x=0.4-0.7$ ) were prepared by sol-gel synthesis. Impedance measurements on pellets of $85 \%$ approximate density sintered at $1300{ }^{\circ} \mathrm{C}$ for $24 \mathrm{~h}$ showed strong evidence of oxide ion conduction with an inclined Warburg spike at low frequencies and capacitance values of $\sim 10^{-6} \mathrm{~F} \mathrm{~cm}^{-1}$ at $40 \mathrm{~Hz}$. Arrhenius plots of total pellet conductivities were linear with activation energies of 1.4-1.56 $\mathrm{eV}$. The conductivity decreased with $x$ and was 2-4 orders of magnitude lower than that with optimized YSZ, $x=0.08$. When the atmosphere was changed from $\mathrm{N}_{2}$ to $\mathrm{O}_{2}$ during impedance measurements, two reversible effects were seen: the Warburg spike contracted greatly, and the sample resistance decreased. These effects were more noticeable at higher $x$ and are attributed to the introduction of p-type electronic conduction, in parallel with the preexisting oxide ion conduction. A similar reversible result was observed upon application of a direct-current $(\mathrm{dc})$ bias during impedance measurements. When either $\mathrm{pO}_{2}$ is increased or a

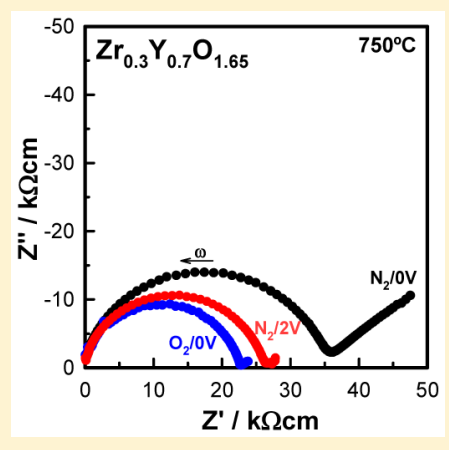
$\mathrm{dc}$ bias is applied, hole creation is believed to arise by the ionization of underbonded oxide ions situated near the $\mathrm{Y}^{3+}$ dopant ions. The ionized electrons are trapped at surface oxygen species, and the holes that are left on oxygen are responsible for p-type conduction. The electrolytic domain of $x=0.4-0.7$ extends up to approximately $10^{-2}$ atm of $\mathrm{O}_{2}$ before p-type conduction is observed. The upper $\mathrm{pO}_{2}$ limit of the electrolytic domain of $x=0.08$ is not known but is likely to be close to or slightly above 1 atm of $\mathrm{O}_{2}$.

\section{INTRODUCTION}

Yttria-stabilized zirconia (YSZ) is the most widely used oxide ion conductor in solid oxide fuel cells (SOFCs) because of its high conductivity, resistance to reduction, good mechanical strength, and stability at high temperatures. ${ }^{1}$ The key to this application is the high concentration of mobile anion vacancies created to charge-compensate for the introduction of $\mathrm{Y}^{3+}$ dopant onto the $\mathrm{Zr}^{4+}$ sites. YSZ is a solid solution series of the general formula $\mathrm{Zr}_{1-x} \mathrm{Y}_{x} \mathrm{O}_{2-x / 2}$, which has a fluorite structure in which the concentration of oxygen vacancies depends on the value of $x$. According to the $\mathrm{ZrO}_{2}-\mathrm{Y}_{2} \mathrm{O}_{3}$ phase diagram, ${ }^{2}$ Figure 1a, the cubic YSZ solid solution extends over the composition range $0<$ $x<0.7$ at high temperatures. At lower $x$ values, the solid solutions transform rapidly to tetragonal and/or monoclinic structures upon cooling. At higher $x$ values, the cubic solid solutions should transform or precipitate secondary phases upon cooling, but transformation rates may be very slow. The coordination numbers of zirconium and yttrium, which are disordered, depend on $x$ and are less than the ideal value of 8 in the fluorite structure. $^{3}$ The cation distribution may not be completely random, however; in composition $x=0.5$, corresponding to the formula $\mathrm{Y}_{0.5} \mathrm{Zr}_{0.5} \mathrm{O}_{1.75}$, there is a preference for a higher coordination number for yttrium of 7.2 compared to 6.8 for zirconium. ${ }^{3}$ The composition $x=0.5$ is sometimes referred to as a pyrochlore, ${ }^{4}$ but diffraction data and crystal structure modeling ${ }^{5}$ show little evidence of a pyrochlore structure apart from this indication of a nonrandom cation distribution.
The composition most widely used in SOFC applications with the highest oxide ion conductivity is $x=0.08$ and is labeled as YSZ08 in this work. ${ }^{1}$ At higher $x$, the oxygen vacancy concentration increases, but defect interactions are held responsible for mobile-ion trapping, leading to a reduction in the conductivity and an increase in the activation energy. ${ }^{6}$ For instance, $x=0.5$ has an oxide ion conductivity of $3.75 \times 10^{-3} \mathrm{~S}$ $\mathrm{cm}^{-1}$ at $800{ }^{\circ} \mathrm{C}$, compared to $2.50 \times 10^{-2} \mathrm{~S} \mathrm{~cm}^{-1}$ for YSZ08 used in SOFCs. ${ }^{1}$

For all oxide-ion-conducting solid electrolytes, the electrolytic domain is limited at low oxygen partial pressures, $\mathrm{pO}_{2}$, by the onset of n-type electronic conduction and at high $\mathrm{pO}_{2}$ by the onset of p-type conduction, as shown schematically in Figure $1 b^{8}$ YSZ08 is generally regarded as stable down to a $\mathrm{pO}_{2}$ value of $10^{-20} \mathrm{~atm}$, which leads to its use as an electrolyte in contact with the anode and reducing atmospheres. Little information is available on the upper $\mathrm{pO}_{2}$ limit of the electrolytic domain for YSZ08, probably because of the difficulty in achieving a wide range of $\mathrm{pO}_{2}$ values greater than atmospheric pressure. Nevertheless, in the p-type region and using Kroger-Vink notation, it is assumed that the uptake of oxygen may occur by a mechanism summarized as

$$
\mathrm{V}_{\mathrm{o}}^{\bullet \bullet}+\frac{1}{2} \mathrm{O}_{2} \rightarrow \mathrm{O}_{\mathrm{o}}^{\mathrm{x}}+2 \mathrm{~h}^{\bullet}
$$

Received: March 14, 2017

Published: May 26, 2017 


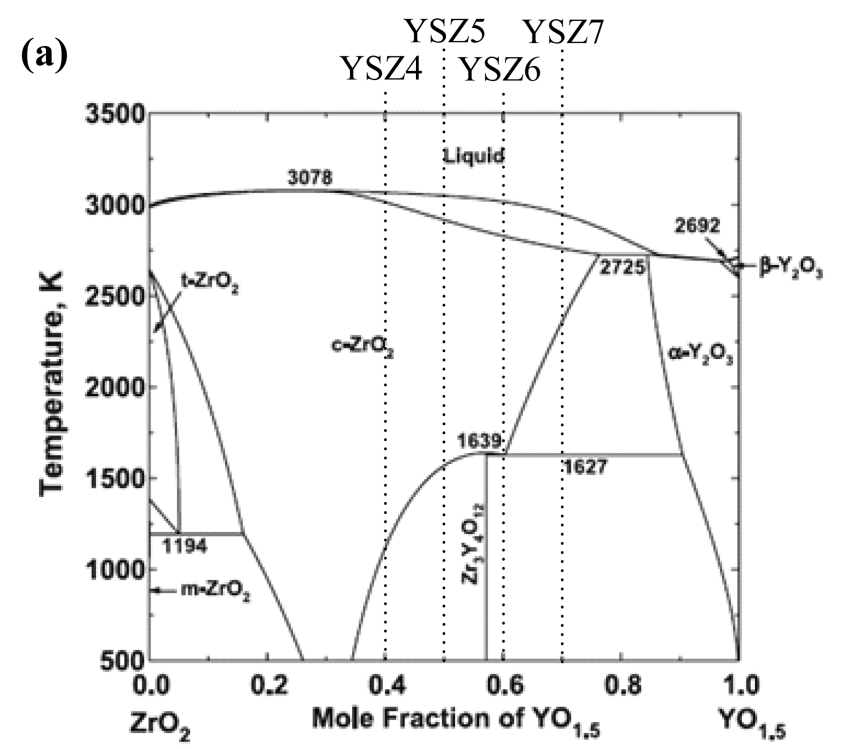

(b)

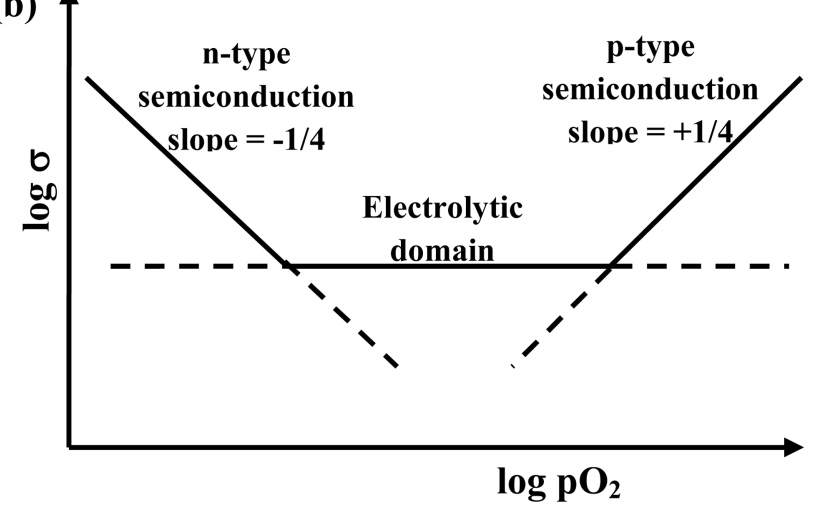

Figure 1. (a) Phase diagram of the $\mathrm{ZrO}_{2}-\mathrm{YO}_{1.5}$ system. ${ }^{2}$ The compositions prepared in this work are indicated. (b) Schematic ionic and electronic conductivity domains as a function of $\mathrm{pO}_{2}$, adapted from ref 8 .

leading to a gradient of $+{ }^{1} / 4$ in plots of $\log \sigma$ versus $\log \mathrm{pO}_{2}$. Usually, holes are assumed to be located on transition-metal ions, present as either controlled dopants or unavoidable impurities, but direct evidence for the site of the hole location seems to not be available.

Recently, Masó and West $^{9}$ demonstrated that electronic conduction is introduced into YSZ08 under the action of a small direct-current $(\mathrm{dc})$ bias at high temperature in air. Similar conductivity changes were seen with acceptor-doped titanate perovskites including calcium-, zinc-, and magnesium-doped $\mathrm{BaTiO}_{3}{ }^{10-12}$ and calcium-doped $\mathrm{BiFeO}_{3},{ }^{13}$ which all show enhanced conductivity with either an increase of $\mathrm{pO}_{2}$ in the measuring atmosphere or the application of a small dc bias. The enhanced conductivity was attributed to the ionization of underbonded $\mathrm{O}^{2-}$ ions in the vicinity of acceptor dopants, leading to hole creation on oxygen, which, conceptually, is the same as the creation of $\mathrm{O}^{-}$ions:

$$
\mathrm{O}_{\mathrm{o}}^{\mathrm{x}} \rightarrow \mathrm{O}_{\mathrm{o}}^{\bullet}+\mathrm{e}^{\prime}
$$

The $\mathrm{O}^{-}$ions are responsible for the enhanced electronic conductivity, which is p-type, because the ionized electrons have been immobilized at sample surfaces by participating in one, or more, of the equilibria

$$
\begin{aligned}
& \mathrm{O}_{2}(\mathrm{~g}) \leftrightarrow \mathrm{O}_{2}(\text { ads }) \leftrightarrow \mathrm{O}_{2}{ }^{-} \leftrightarrow \mathrm{O}_{2}{ }^{2-} / 2 \mathrm{O}^{-} \leftrightarrow 2 \mathrm{O}^{2-} \text { (surface) } \\
& \leftrightarrow 2 \mathrm{O}^{2-} \text { (bulk) }
\end{aligned}
$$

The p-type conduction in the acceptor-doped titanate perovskites is thermally activated, which means that the holes are localized rather than delocalized in the valence band. Therefore, it is a logical step to refer to the holes localized on oxygen as $\mathrm{O}^{-}$ ions.

Although the conductivity of YSZ with $x=0.08$ is not sensitive to $\mathrm{pO}_{2}$ in atmospheres such as $\mathrm{O}_{2}, \mathrm{~N}_{2}$, or air at atmospheric pressure, preliminary tests on compositions with higher $x$ showed that their conductivity is, indeed, sensitive to $\mathrm{pO}_{2}$. The present work reports a systematic study of the conductivity of YSZ with $0.4<x<0.7$ to determine the effects of $\mathrm{pO}_{2}$ and dc bias on the electrical properties.

\section{EXPERIMENTAL SECTION}

Four compositions based on the general formula $\mathrm{Zr}_{1-x} \mathrm{Y}_{x} \mathrm{O}_{2-x / 2}$, with $x=$ $0.4,0.5,0.6$, and 0.7 and labeled YSZ4, YSZ5, YSZ6, and YSZ7, respectively, were prepared by a polymeric sol-gel procedure using $\mathrm{Y}\left(\mathrm{OOCCH}_{3}\right)_{3} \cdot \mathrm{H}_{2} \mathrm{O}(99.9 \%$, Strem Chemicals $)$ and $\mathrm{Zr}$ $\left(\mathrm{OCH}_{2} \mathrm{CH}_{2} \mathrm{CH}_{3}\right)_{4}(70 \%$ Sigma-Aldrich) as precursors. All reagents were of analytical grade and were used without further purification. Absolute ethanol (Scharlab, 99.9\%) was used as a solvent with the necessary amount of acetylacetone, acacH (99\% Alfa Aesar), as a stabilizing agent for the zirconium alkoxide precursor. Zirconium alkoxide was dissolved in an ethanol-acetylacetone mixture (acacH: $\mathrm{Zr}$ molar ratio 4:1), the $\mathrm{Y}\left(\mathrm{OOCCH}_{3}\right)_{3} \cdot \mathrm{H}_{2} \mathrm{O}$ added, and the mixture stirred for $10 \mathrm{~min}$, then transferred to a balloon flask, and heated at $70^{\circ} \mathrm{C}$ for 72 h. A transparent gel formed and was dried in air under an IR lamp at room temperature for approximately 1 day. Finally, the dry gel was ground with an agate mortar and pestle, decomposed by heating at $5{ }^{\circ} \mathrm{C}$ $\mathrm{min}^{-1}$ to $500{ }^{\circ} \mathrm{C}$, left at $500^{\circ} \mathrm{C}$ for $1 \mathrm{~h}$, and then heated to $1300^{\circ} \mathrm{C}$ at a rate of $5{ }^{\circ} \mathrm{C} \mathrm{min}{ }^{-1}$ with a final hold at $1300^{\circ} \mathrm{C}$ for $12 \mathrm{~h}$. Powder samples were cooled, crushed, pressed into pellets of $5 \mathrm{~mm}$ diameter at 1 ton of uniaxial pressing, reheated at $1300{ }^{\circ} \mathrm{C}$ for $24 \mathrm{~h}$, and cooled slowly inside the furnace. The four compositions chosen lie in the fluorite-structure phase field at high temperatures, as shown in Figure 1a. Pellet densities for all compositions were $\sim 85 \%$.

The phase(s) present were analyzed by powder X-ray diffraction (XRD) using a Bruker D4 Endeavor diffractometer, Karlsruhe, Germany, with $\mathrm{Cu} \mathrm{K} \alpha$ radiation. Data were collected by step scanning from $2 \theta=20$ to $70^{\circ}$ with a step size of $0.03^{\circ}$ and $6 \mathrm{~s}$ counting time at each step. The cubic lattice parameter was determined by least-squares refinement for reflections in the range $15<2 \theta<70$, using WinXPow, version 1.06 (Darmstadt, Germany). Scanning electron microscopy (SEM) images of the pellets were taken on a field-emission scanning electron microscope (JEOL 7001F), equipped with a spectrometer for energy-dispersion analysis of X-rays (EDX) from Oxford Instruments, using an acceleration voltage of $15 \mathrm{kV}$. The samples were deposited on an aluminum holder and coated with platinum.

For electrical property measurements, electrodes were fabricated on opposite pellet faces from platinum paste (Pt Ink 6082, Metalor, Barcelona, Spain), which was dried and decomposed by gradual heating to $900{ }^{\circ} \mathrm{C}$. Samples with electrodes attached were placed in a conductivity jig and measured using an Agilent 4294A (Agilent, Madrid, Spain) analyzer over the frequency range of $40 \mathrm{~Hz}$ to $13 \mathrm{MHz}$ and the temperature range of room temperature to $900{ }^{\circ} \mathrm{C}$. Impedance data were corrected for overall pellet geometry and are reported in units of specific resistance $(\Omega \mathrm{cm})$ and capacitance $\left(\mathrm{F} \mathrm{cm}^{-1}\right)$; data were not corrected for the geometry of the regions such as grain boundaries because this was not accurately known. Data were not corrected for the sample porosity. In order to avoid any effect of water, and therefore any possible proton conduction, impedance data were recorded in dry atmospheres. Measurements in atmospheres of different $\mathrm{pO}_{2}$ values were carried out in a specially designed cell fitted with a built-in zirconia 
probe (MicroPoas by SETNAG) next to the sample to measure the $\mathrm{pO}_{2}$ value.

\section{RESULTS}

Pellets fired at $1300{ }^{\circ} \mathrm{C}$ for $24 \mathrm{~h}$ were ground and analyzed by $\mathrm{XRD}$. All samples were single phase and were indexed on a cubic unit cell, space group $F m \overline{3} m$, fully consistent with literature data. XRD data for $x=0.5$ are shown in Figure 2a. There was no
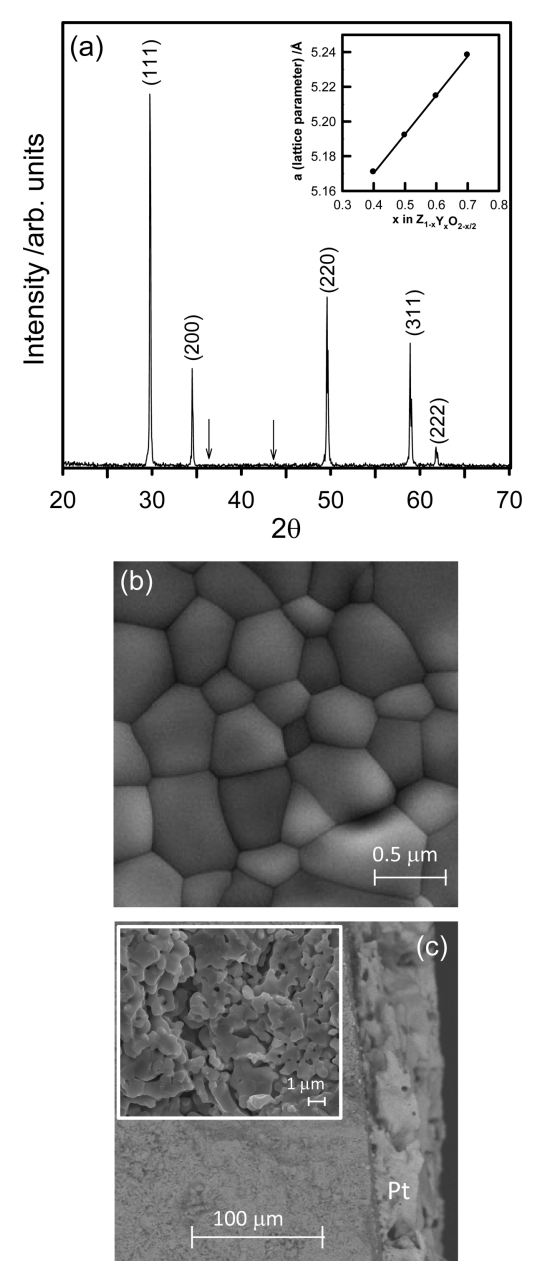

Figure 2. (a) XRD pattern and SEM images of (b) the pellet surface of YSZ5 sintered at $1300{ }^{\circ} \mathrm{C}$ and (c) the fracture surface of YSZ7 with the platinum electrode attached. Inset in part a: Variation in the lattice parameter with $x$. Inset in part c: Magnification of the YSZ7 microstructure of the fracture pellet.

evidence of a possible pyrochlore structure, whose XRD pattern is expected to contain extra peaks (indicated by arrows) at $2 \theta=$ 36.3 and 43.6 compared to the defect fluorite pattern. ${ }^{14}$ Lattice parameters as a function of $x$ (inset of Figure 2a) showed a linear increase with $x$.

The structure of the YSZ solid solution therefore contains a disordered mixture of $\mathrm{Y}^{3+}$ and $\mathrm{Zr}^{4+}$ distributed over the 8coordinate sites of the fluorite structure, with some oxide ion sites vacant, depending on the composition $x$. From Figure 1a, the cubic solid solutions at $1300{ }^{\circ} \mathrm{C}$ are expected to form over the range $0.15 \leq x \leq 0.52 .^{2,15,16}$ Our results showed single-phase cubic solid solutions over a more extensive range of compositions including $x=0.6$ and 0.7 . We do not know at present whether single-phase cubic solid solutions at these compositions are thermodynamically metastable, although clearly they are kineti- cally stable under the conditions of synthesis used here. These results may be consistent with the possibility of forming metastable phases by sol-gel synthesis. ${ }^{17-20}$ A typical microstructure of the pellet surface for YSZ5 is shown in Figure $2 \mathrm{~b}$. Similar microstructures and grain sizes in the range $0.2-0.8 \mu \mathrm{m}$ were observed for all compositions. An SEM image of a fracture surface through a pellet with the platinum electrode attached is shown in Figure 2c for sample YSZ7; the inset of this figure corresponds to a magnification of the YSZ7 microstructure and confirms the expected porosity. A homogeneous layer of the platinum electrode, in contact with the sample surface, is observed in this figure, showing that there is no penetration of the electrode into the interior of the sample. EDX mapping showed a homogeneous distribution of yttrium and zirconium, with no evidence of impurities for any of the compositions.

A typical set of impedance data, recorded in dry $\mathrm{N}_{2}$ at $650{ }^{\circ} \mathrm{C}$ and presented in different formats, are shown in Figure 3 for a pellet of YSZ5 sintered at $1300{ }^{\circ} \mathrm{C}$ for $24 \mathrm{~h}$ with $85 \%$ density. A similar response was observed for all compositions. The impedance complex plane plots (a) show a broad, depressed arc at high frequencies and an inclined spike with some curvature at low frequencies. The total resistance obtained from the intercept of either the arc or the spike on the $Z^{\prime}$ axis had a value of $\sim 20 \mathrm{k} \Omega \mathrm{cm}$. The $Z^{\prime \prime} / M^{\prime \prime}$ spectroscopic plots (b) show peaks at high frequencies that are much broader than expected for an ideal Debye peak, indicating some degree of probable electrical inhomogeneity in the sample but without clear separation of bulk and grain boundary impedances.

The plots of $\log C^{\prime} / \log f$ (Figure $3 \mathrm{c}, \mathrm{d}$ ) for the same data at two temperatures, 650 and $823{ }^{\circ} \mathrm{C}$, show evidence for a highfrequency plateau of the value $\sim 10 \mathrm{pF}$, which corresponds to the limiting high-frequency permittivity of $\sim 112$. The $C^{\prime}$ values increase with decreasing frequency, with some evidence for a poorly resolved intermediate plateau at $\sim 30 \mathrm{pF}$. This value is too small for it to represent a conventional grain boundary because it corresponds to a significant volume fraction of the sample. Instead, we attribute it to a combination of either a constriction grain boundary associated with the sample porosity and possible inhomogeneity in the distribution of the $\mathrm{Y}^{3+}$ dopant or a dipole orientation-related impedance such as that reported for YSZ08. ${ }^{21}$ Further studies are in progress to understand the nature of this impedance.

With decreasing frequency, $C^{\prime}$ increases rapidly to reach a value of approximately $1 \mu \mathrm{F}$ at $40 \mathrm{~Hz}$ and $823{ }^{\circ} \mathrm{C}$ (Figure $3 \mathrm{~d}$ ); this value is typical of ion blocking at a sample-electrode interface and the formation of a double-layer capacitance. The inclined spike shown in Figure $3 a$ at low frequencies is attributed to the diffusion of oxygen molecules toward and away from sample-electrode interfaces, which therefore places a limit on the rate of oxygen-exchange reactions that occur during lowfrequency measurements. Although this spike, attributed to a Warburg impedance, is not directly associated with oxide ion conduction, it is, nevertheless, an intrinsic component of redox reactions involving oxygen and is an indirect indication of oxide ion conduction. We therefore interpret the low-frequency impedance data to indicate that conduction is primarily by oxide ions but with the possibility of a small amount of electronic conduction (see later).

From data such as these, total sample resistances were obtained from intercepts of either the arc or the Warburg spike on the $Z^{\prime}$ axis and are shown as conductivity Arrhenius plots as a function of the reciprocal temperature in Figure 4; literature data for YSZ, $x=0.08,{ }^{22}$ are shown for comparison. The total 

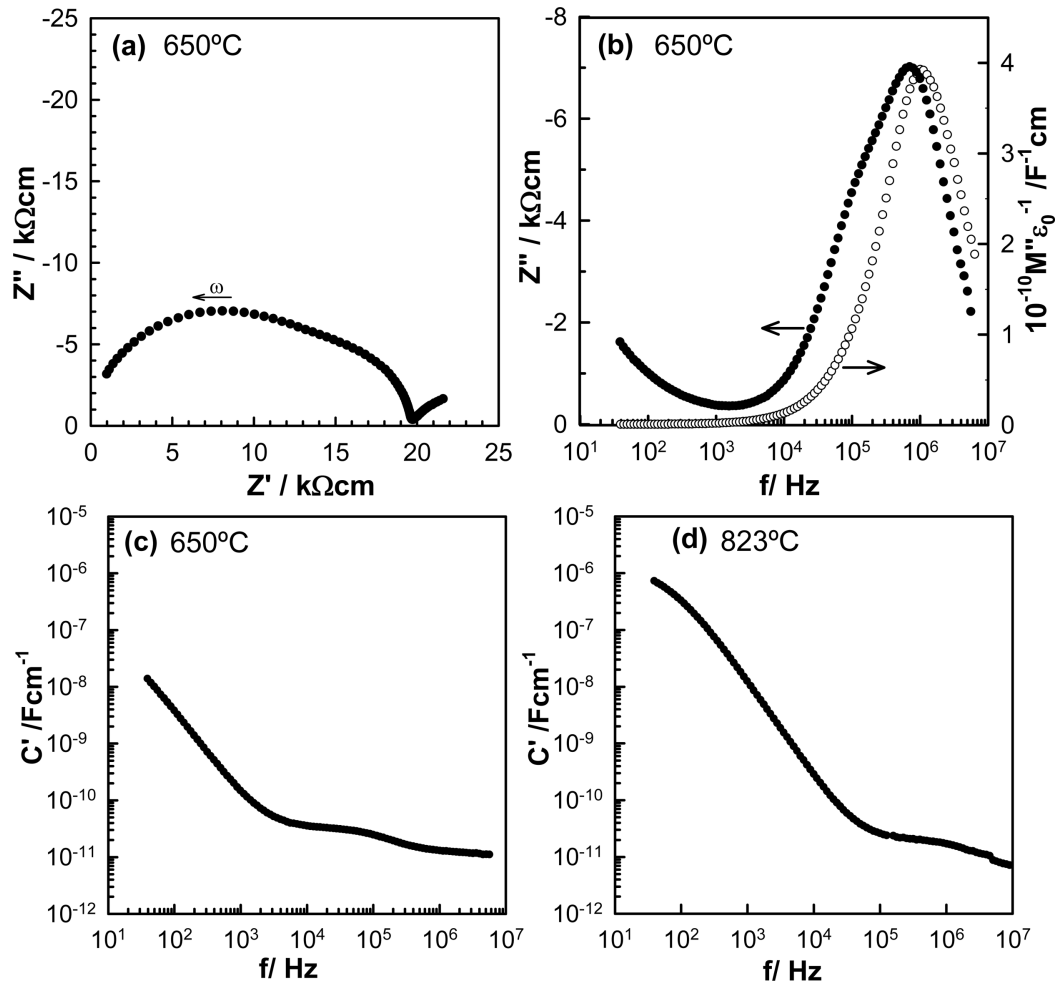

Figure 3. Impedance complex plane plots of (a) $Z^{*}$ and (b) $Z^{\prime \prime} / M^{\prime \prime}$ and (c and d) $C^{\prime}$ spectroscopic plots at different temperatures for YSZ5. Measurements are in dry $\mathrm{N}_{2} ; \omega=2 \pi f$.

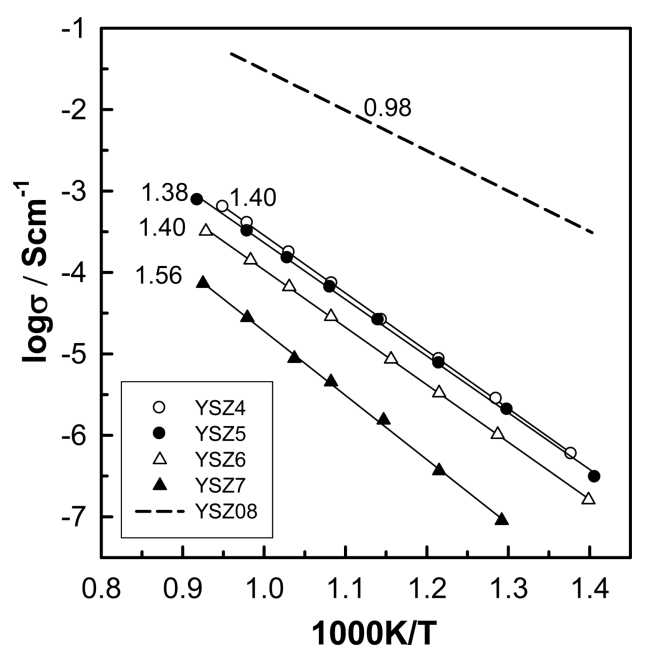

Figure 4. Arrhenius plots of the total conductivities for samples sintered at $1300{ }^{\circ} \mathrm{C}$ and measured in dry $\mathrm{N}_{2}$. Error bars are within the size of the data points. The activation energies $(\mathrm{eV})$, with errors in the range $0.02-$ $0.05 \mathrm{eV}$, are shown beside each data set. The YSZ08 ceramic sintered at $1600{ }^{\circ} \mathrm{C}$ for $6 \mathrm{~h}$ is included for comparison. ${ }^{22}$

conductivity of YSZ solid solutions decreases with $x$ and is $2-4$ orders of magnitude lower than that of YSZ with $x=0.08$. In addition, the activation energy increases with $x$. It is possible that the bulk conductivities are somewhat higher than the total conductivities shown in Figure 4 because either grain-boundaryconstriction or dipole-reorientation impedances are included in the total resistance values; also, conductivities were not corrected for the sample porosity. The impedance measurements were obtained upon both cooling and heating, and the data were reproducible, showing linear behavior.
The data shown in Figures 3 and 4 were recorded in an atmosphere of dry $\mathrm{N}_{2}$ with a $\mathrm{pO}_{2}$ value of approximately $10^{-4}$ atm. Data are shown in Figure 5 for pellets of each composition measured in different atmospheres. Data were measured first in dry $\mathrm{N}_{2}\left[\mathrm{pO}_{2} \sim(1-2) \times 10^{-4}\right.$ atm $]$; the atmosphere was then changed to dry $\mathrm{O}_{2}\left(\mathrm{pO}_{2} \sim 1 \mathrm{~atm}\right)$ and the system allowed to stabilize for a few minutes before impedance data were collected in $\mathrm{O}_{2}$. Two effects are seen in the impedance complex plane plots (Figure $5 \mathrm{a}-\mathrm{d}$ ), which were reversible upon switching between $\mathrm{O}_{2}$ and $\mathrm{N}_{2}$. First, the Warburg spike contracts greatly and becomes a small arc in $\mathrm{O}_{2}$; second, the total sample resistance decreases. The effects were more noticeable with higher $x$ compositions. It is most unlikely that these two phenomena could be attributed to changes in the oxide ion conductivity in the samples simply as a consequence of changing $\mathrm{pO}_{2}$ in the measuring atmosphere.

Changes in the conductivity with $\mathrm{pO}_{2}$ are widely used as an indication of extrinsic electronic conduction in materials because reactions such as eq 1 lead to changes in the electronic carrier concentration of either electrons or holes. We, therefore, interpret the data shown in Figure 5 to indicate the presence of p-type conduction when samples are measured in $\mathrm{O}_{2}$ because chemisorption of oxygen with increasing $\mathrm{pO}_{2}$ leads to hole creation (eq 1). This means that a certain amount of p-type electronic conduction ${ }^{23,24}$ occurs in parallel with the preexisting oxide ion conduction. The electronic conduction must arise from reactions involving $\mathrm{O}_{2}$ in the atmosphere. Equation 1 shows the creation of holes but gives no indication as to their location. Traditionally, holes are associated with transition-metal impurities, but we believe that a more likely location, at least in our materials, is underbonded oxide ions.

The loss of the Warburg spike is also attributed to the creation of a parallel electronic conduction pathway. ${ }^{9}$ It is further emphasized in the capacitance data (insets in Figure $5 a-d$ ), in 

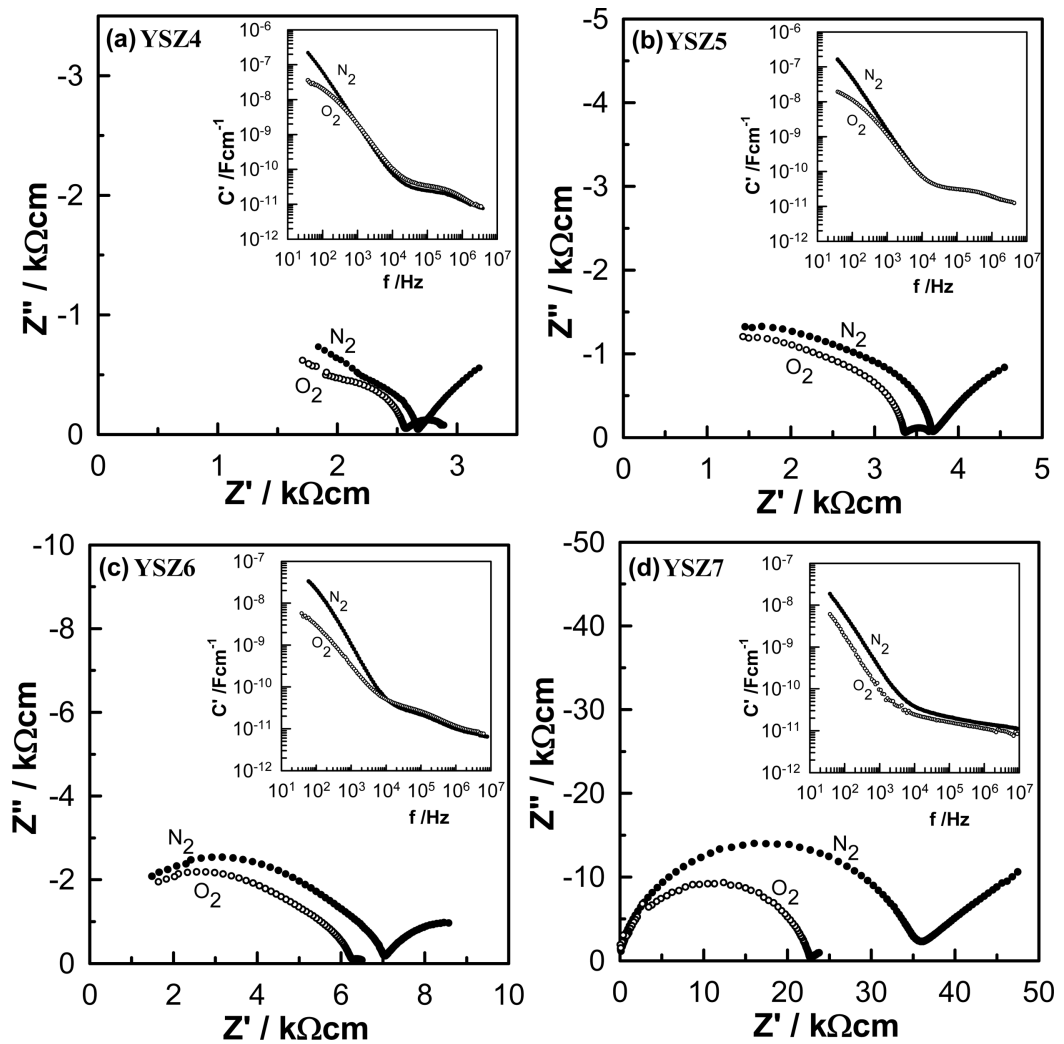

Figure 5. Impedance complex plane plots, $Z^{*}$, with $C^{\prime}$ spectroscopic plots as insets for all compositions measured in dry $\mathrm{N}_{2}$ and $\mathrm{O}_{2}$ at $750{ }^{\circ} \mathrm{C}$.

which the large, high-valued low-frequency capacitance decreases because it is no longer associated specifically with charge transfer at the thin, double-layer interface.

Impedance data were sensitive to $\mathrm{pO}_{2}$ over the complete temperature range studied, $500-850^{\circ} \mathrm{C}$, and are summarized in Arrhenius plots of the total conductivity for one composition, YSZ7, in Figure 6. The data recorded in $\mathrm{O}_{2}$ are higher by a factor of $2-3$ with a smaller activation energy and clearly must contain a contribution of both electronic and ionic conductivities. We do not know whether the data in $\mathrm{N}_{2}$ also contain a small electronic component or whether they represent exclusively ionic conduction. These results show that, with reference to Figure 1b, samples YSZ4-YSZ7 in air at atmospheric pressure are in the

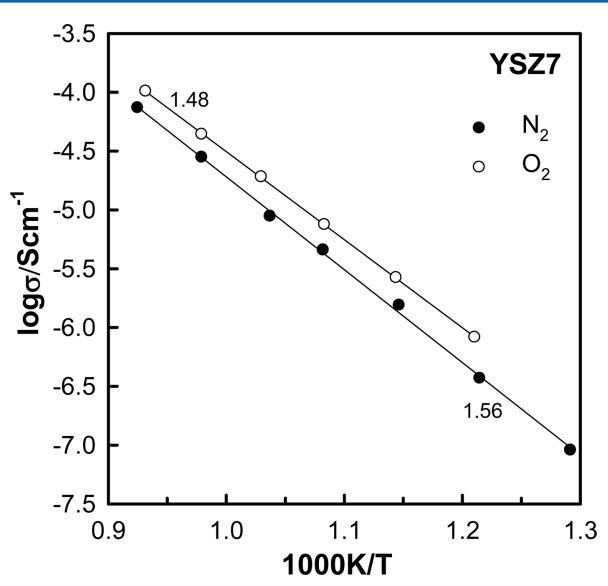

Figure 6. Arrhenius plots of the total conductivity for YSZ7 measured in dry $\mathrm{N}_{2}$ and $\mathrm{O}_{2}$. The activation energies $(\mathrm{eV})$ are shown beside each data set. crossover region between the electrolytic domain and p-type conduction. At present, we have not performed a detailed determination of the electronic transport number in our compositions, which would allow the contribution of each conduction mechanism to the electrical response to be determined as a function of the temperature, $\mathrm{pO}_{2}$ and $x$. Different methods are reported in the literature to determine the electronic and ionic components of the conductivity. 9,25

The effect on the impedance data of the application of a small $\mathrm{dc}$ bias, in the range $0.5-2 \mathrm{~V}$, was found to be very similar to that of increasing $\mathrm{pO}_{2}$. A selection of results is shown in Figure 7 for YSZ7 and YSZ4. In Figure 7a, data are shown in $\mathrm{N}_{2}$ and $\mathrm{O}_{2}$ without an applied bias and in $\mathrm{N}_{2}$ with $2 \mathrm{~V}$ bias. The data in $\mathrm{O}_{2}$ and $\mathrm{N}_{2}$ with $2 \mathrm{~V}$ bias are similar, and both show reduced total resistance and a much reduced Warburg spike. In Figure $7 \mathrm{~b}$, data are shown for $\mathrm{dc}$ bias in the range $1-10 \mathrm{~V}$; a systematic decrease in both the sample resistance and Warburg impedance is observed.

The changes in the conductivity with changing $\mathrm{pO}_{2}$ are not instantaneous but take a few minutes to reach the steady state, as shown in Figure 8 for YSZ5 at $750{ }^{\circ} \mathrm{C}$. The sample was first equilibrated in $\mathrm{N}_{2}$ for $24 \mathrm{~h}$ and the resistance measured as a function of time on switching to $\mathrm{O}_{2}$. Once a steady state had been reached, the atmosphere was switched back to $\mathrm{N}_{2}$; several minutes were needed to reach a steady state in both directions. Figure 8 therefore corresponds to a continuous time plot with a switch in the atmosphere at intermediate time.

\section{DISCUSSION}

The electrical properties of YSZ, $x=0.4-0.7$, without application of a dc bias, are consistent with oxide ion conduction, as is, of course, widely recognized for YSZ08. ${ }^{26}$ The conductivity is lower than that of YSZ08, which is usually used as a ceramic electrolyte 

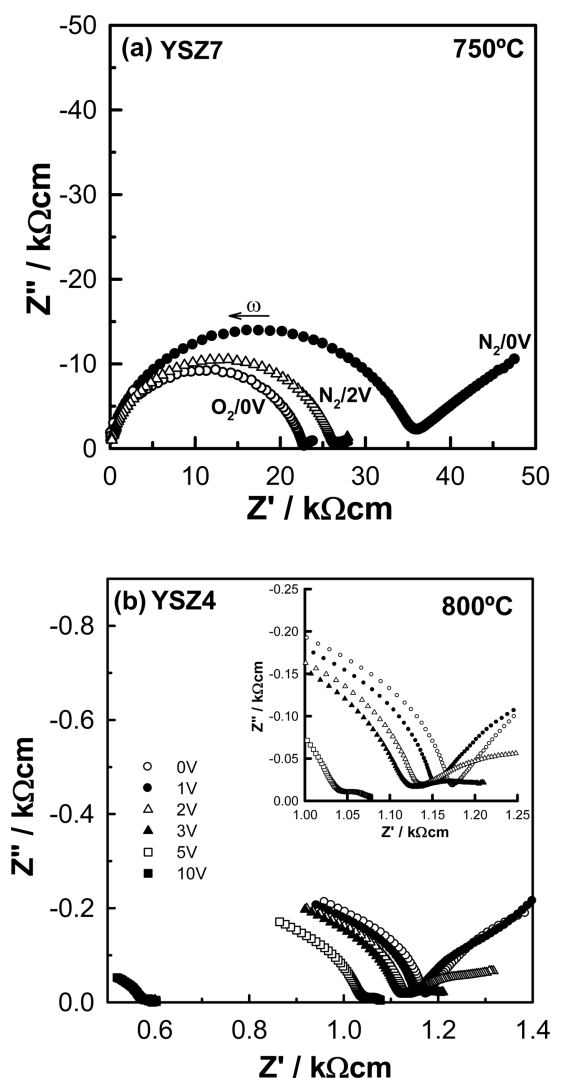

Figure 7. (a) Impedance complex plane plots for YSZ7 measured in dry $\mathrm{N}_{2}$ and $\mathrm{O}_{2}$ at $750{ }^{\circ} \mathrm{C}$ and with $2 \mathrm{~V}$ bias voltage in dry $\mathrm{N}_{2}$. (b) Impedance complex plane plots for YSZ4 measured in dry $\mathrm{N}_{2}$ after different bias voltages were applied at $800^{\circ} \mathrm{C}$.

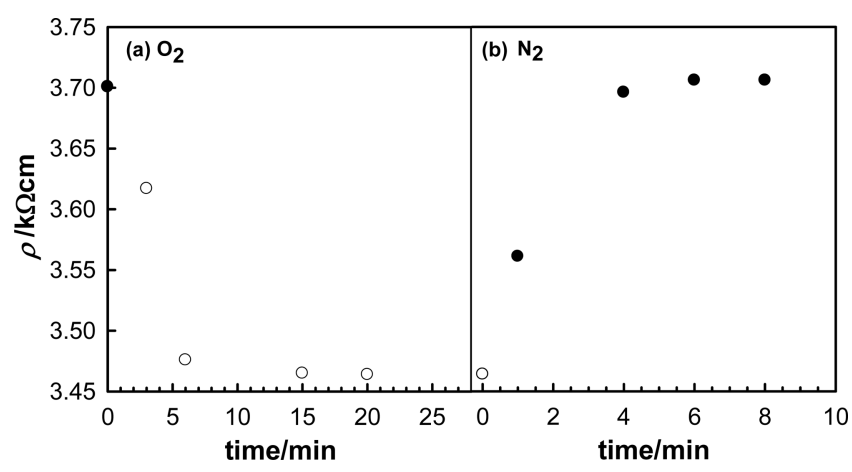

Figure 8. Time-dependent total resistivity for YSZ5 at $750{ }^{\circ} \mathrm{C}$ in dry (a) $\mathrm{O}_{2}$ and (b) $\mathrm{N}_{2}$.

in SOFC systems. Although the compositions prepared with higher $x$ have a higher concentration of oxygen vacancies, the reduction in the ionic conductivity with increasing $x$ is attributed to the trapping of oxygen vacancies in defect complexes associated with the $\mathrm{Y}^{3+}$ dopant; this is also consistent with the observed increase in the activation energy for ionic conduction in higher $x$ compositions. ${ }^{6}$

YSZ08 is used as the ceramic electrolyte in SOFCs, in part because it is very stable to reduction. However, it has recently been shown that electronic conduction can be induced in YSZ08 by the application of a small bias voltage. ${ }^{9}$ This causes YSZ08 to become a mixed conductor with, for instance, an electronic transport number, $t_{\mathrm{e}}$, of 0.5 with $10 \mathrm{~V}$ bias at $\sim 550{ }^{\circ} \mathrm{C}$. The electronic conductivity is lost upon removal of the dc bias.
In the present study, we report a similar dependence of the conductivity on the dc bias but also show that the conductivity is sensitive to $\mathrm{pO}_{2}$. The onset of the electronic conductivity is shown by two changes to the impedance data: first, the Warburg spike is greatly reduced and, second, the total sample resistance is reduced. Reversible changes of a similar nature are seen with both an increase in $\mathrm{pO}_{2}$ and a dc bias. There is no evidence from the literature that YSZ08 is sensitive to an increase in $\mathrm{pO}_{2}$, although it is sensitive to dc bias. ${ }^{9}$ From the results presented here, the introduction of the electronic conductivity becomes easier with increasing $x$, and it seems highly probable, therefore, that YSZ08 itself will be sensitive to $\mathrm{pO}_{2}$ at pressures just above 1 atm of $\mathrm{O}_{2}$. This result has clear significance for the use of YSZ-based materials either in an $\mathrm{O}_{2}$-rich atmosphere or in the presence of a bias voltage.

The sensitivity of the total sample conductivity to changes in $\mathrm{pO}_{2}$ (Figure 5) is evidence that the electronic conduction mechanism is p-type. This was presumed, but not established, in the earlier YSZ results ${ }^{9}$ and follows previous studies of acceptordoped titanate perovskites that showed enhanced p-type conductivity with a dc bias. In those studies, ${ }^{9-12}$ the mechanism of hole creation was attributed to the ionization of underbonded oxide ions associated with lower-valence acceptor dopants. The holes that are created are localized on oxygen, as $\mathrm{O}^{-}$ions, and are responsible for the thermally activated p-type conduction. The $\mathrm{Y}^{3+}$ component of the YSZ solid solutions may be regarded also as an acceptor dopant in which $\mathrm{Y}^{3+}$ substitutes for $\mathrm{Zr}^{4+}$; oxide ions in the immediate vicinity of $\mathrm{Y}^{3+}$ are effectively underbonded and can be readily ionized. The effect of increasing $x$ is to increase the $\mathrm{Y}^{3+}$ concentration and, therefore, the concentration of underbonded oxide ions; this may account for the increased sensitivity to $\mathrm{pO}_{2}$ that is seen with increasing $x$.

The chemisorption on sample surfaces that takes place with increasing $\mathrm{pO}_{2}$ is a commonly used diagnostic for extrinsic conductivity and to distinguish $\mathrm{p}$-type from n-type conduction. Using eq 1 to represent the chemisorption, electrons are removed from the sample, presumably close to the absorption sites, to form reduced oxygen species. In reality, several equilibria at sample surfaces may be involved, as indicated schematically in eq 3 , giving rise to species such as superoxide $\mathrm{O}_{2}{ }^{-}$, peroxide $\mathrm{O}_{2}{ }^{2-}$, $\mathrm{O}^{-}$, and finally $\mathrm{O}^{2-}$ ions, whose net negative charges are balanced by the holes that are created as lattice $\mathrm{O}^{-}$ions. Several of these equilibria require electrons and, hence, with increasing $\mathrm{pO}_{2}$, lead to an overall increase in the p-type conductivity, as shown in Figure 8. These processes commence at any part of sample surface exposed to the atmosphere.

Behavior similar to that seen with increasing $\mathrm{pO}_{2}$ is observed upon application of a small dc bias, which, for voltages of $1-2 \mathrm{~V}$, is below the probable decomposition voltage of the samples. This behavior may also be interpreted in terms of the equilibria shown in eq 3 because these equilibria are driven to the right-hand side by a positive bias, leading to the removal of electrons from the sample and an increase in p-type conduction. The positive electrode is therefore responsible for the observed conductivity changes, and there is no evidence of any opposite effect occurring at the negative electrode, which would lead to negative charge injection. Thus, if charge injection were to occur, this would lead to the onset of n-type conduction, as represented by the crossover region at low $\mathrm{pO}_{2}$ shown in Figure $1 \mathrm{~b}$, in contrast to the observed p-type behavior. There are reported instances of blackening and electronic conduction in YSZ ceramics that can be attributed to charge injection from the negative electrode under highly reducing conditions. If changes to the sample do 
occur at the negative electrode under dc bias, they are not directly responsible for the effects reported here. Thus, we also exclude the possibility of Schottky barrier formation and charge injection resulting from a reduction in the barrier height upon application of the dc bias.

\section{CONCLUSIONS}

There is huge interest in YSZ as the ceramic electrolyte for SOFC applications because, as well as its high oxide ion conductivity, it is very resistant to reduction; consequently, its "electrolytic window" extends down to very low partial pressures of oxygen before it shows the onset of n-type conduction. This work concerns the upper limit of the electrolytic window before the onset of p-type conduction.

Conductivity measurements in different atmospheres show that, with increasing yttrium content, YSZ compositions $x=0.4-$ 0.7 are already at the crossover between electrolytic and p-type conduction upon switching between $\mathrm{N}_{2}$ and $\mathrm{O}_{2}$ atmospheres; a similar conclusion is reached from impedance measurements in the presence of a small dc bias and is attributed to the creation of holes on underbonded $\mathrm{O}^{2-}$ ions associated with the $\mathrm{Y}^{3+}$ acceptor dopant. We do not know the value of the activation energy for the electronic component in the p-type region, but it is probably nonzero, which means that the holes are localized on oxygen and not delocalized in the valence-band structure. The activation energy of YSZ7 in $\mathrm{O}_{2}, 1.48 \mathrm{eV}$, is somewhat less than that in $\mathrm{N}_{2}$, $1.56 \mathrm{eV}$ (Figure 6), and, therefore, the activation energy for $\mathrm{p}$ type conduction is presumed to be less than that for oxide ion conduction.

These results are significant for several reasons:

First, they demonstrate that, depending on the yttrium content, YSZ samples may lose their exclusive oxide ion conductivity either in the presence of a small applied voltage or with increasing oxygen partial pressure. For samples of YSZ08 used in SOFC applications, the onset of p-type conduction is likely to occur at oxygen partial pressures close to, or slightly above, 1 atm.

Second, they demonstrate an alternative method by which electronic conductivity can be introduced into YSZ. Most focus in the literature is on the onset of possible $n$-type conductivity with either reducing atmospheres or decreasing $\mathrm{pO}_{2}$, whereas we demonstrate the very easy introduction of p-type conductivity under near-ambient conditions and in the presence of small dc voltages.

Third, they highlight that the mechanism of hole creation must involve redox activity of underbonded oxide ions, which challenges the widely held belief that oxygen in oxides is present solely as the double-negative oxidation state. In high-purity chemicals such as those used here, there seems to be no other possibility for location of the holes.

Fourth, the ready introduction of p-type conductivity into YSZ-based materials may have significant consequences for their application as electronically insulating ceramics either in a high$\mathrm{pO}_{2}$ environment or in the presence of extraneous voltages.

\section{AUTHOR INFORMATION}

\section{Corresponding Author}

*E-mail:mir@uji.es.

\section{ORCID 1}

Héctor Beltrán-Mir: 0000-0002-7836-1602

Notes

The authors declare no competing financial interest.

\section{ACKNOWLEDGMENTS}

M.J., H.B.-M., and E.C. thank the Universidad Jaume I (Project UJI-B2016-38) and Ministerio de Economía, Industria y Competitividad (Project MAT2016-80410-P), for financial support. M.J. also thanks the Universidad Jaume I for a fellowship.

\section{REFERENCES}

(1) Stambouli, A. B.; Traversa, E. Solid oxide fuel cells (SOFCs): a review of an environmentally clean and efficient source of energy. Renewable Sustainable Energy Rev. 2002, 6, 433-455.

(2) Chen, M.; Hallstedt, B.; Gauckler, L. J. Thermodynamic modeling of the $\mathrm{ZrO}_{2}-\mathrm{YO}_{15}$ system. Solid State Ionics 2004, 170, 255-274.

(3) Zhang, Z.; Middleburgh, S. C.; De los Reyes, M.; Lumpkin, G. R.; Kennedy, B. J.; Blanchard, P. E. R.; Reynolds, E.; Jang, L. Y. Gradual Structural Evolution from Pyrochlore to Defect-Fluorite in $\mathrm{Y}_{2} \mathrm{Sn}_{2-x} \mathrm{Zr}_{x} \mathrm{O}_{7}$ : Average vs Local Structure. J. Phys. Chem. C 2013, 117, 26740-26749.

(4) Panero, W.; Stixrude, L.; Ewing, R. First-principles calculation of defect-formation energies in the $\mathrm{Y}_{2}(\mathrm{Ti}, \mathrm{Sn}, \mathrm{Zr})_{2} \mathrm{O}_{7}$ pyrochlore. Phys. Rev. B: Condens. Matter Mater. Phys. 2004, 70, 054110-1-054110-11.

(5) Du, Q.; Zhou, G.; Zhou, J.; Jia, X.; Zhou, H. Enhanced luminescence of novel $\mathrm{Y}_{2} \mathrm{Zr}_{2} \mathrm{O}_{7}: \mathrm{Dy}^{3+}$ phosphors by $\mathrm{Li}^{+}$co-doping. J. Alloys Compd. 2013, 552, 152-156.

(6) Kilner, J. A.; Steele, B. C. H. Nonstoichiometric Oxides; Academic Press, 1981.

(7) Kumar, M.; Raj, I. A.; Pattabiraman, R. $\mathrm{Y}_{2} \mathrm{Zr}_{2} \mathrm{O}_{7}$ (YZ)-pyrochlore based oxide as an electrolyte material for intermediate temperature solid oxide fuel cells (ITSOFCs)-Influence of Mn addition on YZ. Mater. Chem. Phys. 2008, 108, 102-108.

(8) West, A. R. Solid State Chemistry and its Applications, 2nd ed.; Wiley, 2014.

(9) Masó, N.; West, A. R. Electronic Conductivity in Yttria-Stabilized Zirconia under a Small dc Bias. Chem. Mater. 2015, 27, 1552-1558.

(10) Masó, N.; Prades, M.; Beltrán, H.; Cordoncillo, E.; Sinclair, D. C.; West, A. R. Field enhanced bulk conductivity of acceptor-doped $\mathrm{BaTi}_{1-\mathrm{x}} \mathrm{Ca}_{\mathrm{x}} \mathrm{O}_{3-\mathrm{x}}$ ceramics. Appl. Phys. Lett. 2010, 97, 062907-1-0629073 .

(11) Beltrán, H.; Prades, M.; Masó, N.; Cordoncillo, E.; West, A. R. Voltage-Dependent Low-Field Bulk Resistivity in $\mathrm{BaTiO}_{3}: \mathrm{Zn}$ Ceramics. J. Am. Ceram. Soc. 2010, 93, 500-505.

(12) Prades, M.; Masó, N.; Beltrán, H.; Cordoncillo, E.; West, A. R. Field enhanced bulk conductivity of $\mathrm{BaTiO}_{3}: \mathrm{Mg}$ ceramics. J. Mater. Chem. 2010, 20, 5335-5344.

(13) Masó, N.; Beltrán, H.; Prades, M.; Cordoncillo, E.; West, A. R. Field-enhanced bulk conductivity and resistive-switching in Ca-doped $\mathrm{BiFeO}_{3}$ ceramics. Phys. Chem. Chem. Phys. 2014, 16, 19408-19416.

(14) Zou, X.; Zhou, G.; Yi, H.; Zhang, G.; Wang, S. Fabrication of Transparent $\mathrm{Y}_{2} \mathrm{Zr}_{2} \mathrm{O}_{7}$ Ceramics from Combustion-Synthesized Powders. J. Am. Ceram. Soc. 2011, 94, 1002-1004.

(15) Du, Y.; Jin, Z.; Huang, P. Thermodynamic Assessment of the $\mathrm{ZrO}_{2}-\mathrm{YO}_{1.5}$ System. J. Am. Ceram. Soc. 1991, 74, 1569-1577.

(16) Subramanian, M. A.; Aravamudan, G.; Subba Rao, G. V. Oxide pyrochlores - A review. Prog. Solid State Chem. 1983, 15, 55-143.

(17) Brinker, C. J.; Scherer, G. W. Sol-Gel Science; Academic Press: New York, 1990.

(18) Mackenzie, J. D. Unusual non-crystalline solids from gels in 2004. J. Non-Cryst. Solids 1985, 73, 631-637.

(19) Viazzi, C.; Bonino, J. P.; Ansart, F.; Barnabé, A. Structural study of metastable tetragonal YSZ powders produced via a sol-gel route. J. Alloys Compd. 2008, 452, 377-383.

(20) Beltrán, H.; Gómez, B.; Masó, N.; Cordoncillo, E.; West, A. R. Electrical properties of ferroelectric $\mathrm{BaTi}_{2} \mathrm{O}_{5}$ and dielectric $\mathrm{Ba}_{6} \mathrm{Ti}_{17} \mathrm{O}_{40}$ ceramics. J. Appl. Phys. 2005, 97, 084104-1-084104-6.

(21) Hernandez, M. A.; West, A. R. Dipolar relaxation and impedance of an yttria-stabilised zirconia ceramic electrolyte. J. Mater. Chem. A 2016, 4, 1298-1304. 
(22) Ren, P.; Masó, N.; Liu, Y.; Ma, L.; Fan, H.; West, A. R. Mixed oxide ion and proton conduction and p-type semiconduction in $\mathrm{BaTi}_{0.98} \mathrm{Ca}_{0.02} \mathrm{O}_{2.98}$ ceramics. J. Mater. Chem. C 2013, 1, 2426-2432.

(23) Jamnik, J.; Maier, J. J. Treatment of the Impedance of Mixed Conductors Equivalent Circuit Model and Explicit Approximate Solutions. J. Electrochem. Soc. 1999, 146, 4183-4188.

(24) Lai, W.; Haile, S. M. Impedance spectroscopy as a tool for chemical and electrochemical analysis of mixed conductors: a case study of ceria. J. Am. Ceram. Soc. 2005, 88, 2979-2997.

(25) Huggins, R. A. Simple method to determine electronic and ionic components of the conductivity in mixed conductors. Ionics 2002, 8, 300-313.

(26) Brune, A.; Lajavardi, M.; Fisler, D.; Wagner, J. B., Jr. The electrical conductivity of yttria-stabilized zirconia prepared by precipitation from inorganic aqueous solutions. Solid State Ionics 1998, 106, 89-101. 


\subsection{Discusión de los resultados}

En el presente estudio se han sintetizado diferentes composiciones en el sistema $\mathrm{ZrO}_{2}-\mathrm{YO}_{1.5}$, con estequiometría $\mathrm{Zr}_{1-\mathrm{x}} \mathrm{Y}_{\mathrm{x}} \mathrm{O}_{2-\mathrm{x} / 2}$ con valores de $\mathrm{x}>0.4$. La metodología de síntesis utilizada ha sido la de sol-gel polimérico, usando como precursores el acetato de itrio y el n-propóxido de circonio. Posteriormente las muestras se calcinaron a $1300^{\circ} \mathrm{C}$ durante $12 \mathrm{~h}$. Los resultados de difracción de rayos X (DRX) evidenciaron la formación de disoluciones sólidas con un contenido molar del 40\% (YSZ4), 50\% (YSZ5), 60\% (YSZ6) y 70\% (YSZ7) en itrio respectivamente tal como muestra la siguiente tabla (La figura 1a, del artículo muestra las composiciones en el diagrama de equilibrio de fases).

Tabla 5. Composiciones preparadas basadas en $\mathrm{Zr}_{1-\mathrm{x}} \mathrm{Y}_{\mathrm{x}} \mathrm{O}_{2-\mathrm{x} / 2}$.

\begin{tabular}{|c|c|c} 
Referencia & $\mathbf{x}$ & Composición \\
\hline YSZ4 & 0.4 & $\mathrm{Zr}_{0.6} \mathrm{Y}_{0.4} \mathrm{O}_{1.8}$ \\
YSZ5 & 0.5 & $\mathrm{Zr}_{0.5} \mathrm{Y}_{0.5} \mathrm{O}_{1.75}$ \\
YSZ6 & 0.6 & $\mathrm{Zr}_{0.4} \mathrm{Y}_{0.6} \mathrm{O}_{1.70}$ \\
YSZ7 & 0.7 & $\mathrm{Zr}_{0.3} \mathrm{Y}_{0.7} \mathrm{O}_{1.65}$
\end{tabular}

A concentraciones de itrio superiores al $70 \%$ molar se obtuvo fase secundaria.

Todas las disoluciones sólidas presentaron fase cúbica con estructura de fluorita defectuosa, estableciendo por tanto el límite de la disolución sólida en un 70\% molar de itrio. La formación de fases cúbicas en el intervalo de composiciones $\mathrm{x}=0.4-0.7$, es consistente con la posibilidad de formar estructuras metaestables mediante la metodología de síntesis sol-gel.

Para el estudio de sus propiedades eléctricas, se realizaron pastillas $5 \mathrm{~mm}$ del material en polvo calcinado a $1300^{\circ} \mathrm{C}$, mediante la aplicación uniaxial de una 
tonelada y se les realizó un tratamiento térmico a $1300^{\circ} \mathrm{C}$ durante $24 \mathrm{~h}$. Las pastillas obtenidas presentaron una densidad relativa de alrededor del 85\%.

La caracterización eléctrica de las muestras se realizó mediante la técnica de espectroscopia de impedancia. Inicialmente se realizaron medidas de impedancia en atmósfera de $\mathrm{N}_{2}$, a diferentes temperaturas para todas las composiciones, obteniendo unos resultados muy similares en todas las composiciones. Se observó en todas las muestras la existencia de una cierta inhomogeneidad, posiblemente atribuida a una diferencia de comportamiento eléctrico entre el bulk y las fronteras de grano. Esta inhomogeneidad también se observa en los valores de las capacidades (figura 3c del artículo) a frecuencias medias, pudiendo ser causada por la porosidad de la muestra o a la distribución heterogénea del $\mathrm{Y}^{3+}$ en la muestra. También se podría considerar la presencia de dipolos, tal y como se ha documentado en YSZ08 [183].

Por otra parte, se observó la presencia de un "spike" que se atribuye a la difusión del oxígeno en la interfase muestra-electrodo, siendo un claro indicio de conducción iónica (figura 3a y d, del artículo).

Adicionalmente se realizaron medidas de impedancia para todas las composiciones en el intervalo de temperaturas entre 850 y $400^{\circ} \mathrm{C}$, en atmósfera de $\mathrm{N}_{2}$ con el objetivo de obtener los gráficos de Arrhenius. Los datos mostrados en estos gráficos, han sido extraídos de la conductividad total de Z* (la intercepción del arco en el eje Z') y comparados con el YSZ08. Las conductividades totales exhibidas por las composiciones sintetizadas en el presente trabajo presentan valores dos órdenes de magnitud por debajo de las conductividades de YSZ08, tal y como se esperaba para disoluciones sólidas con concentraciones mayores de itrio. Por otra parte, hay que resaltar el aumento de la energía de activación con el aumento de la cantidad de itrio presente en la estructura. Este aumento es consistente con la formación de complejos entre las vacantes de oxígeno creadas y 
los iones $\mathrm{Y}^{3+}$, conllevando un aumento de la energía de activación y una disminución de la conductividad iónica en este caso [184].

Con el objetivo de estudiar como depende la conductividad de las muestras con diferentes atmósferas parciales de oxígeno, se han realizado medidas también en atmósfera de $\mathrm{O}_{2}$ seco, y han sido comparadas con las de $\mathrm{N}_{2}$ para todas las composiciones. De las medidas obtenidas se pueden destacar dos tendencias, el colapso del "spike" de bajas frecuencias y el aumento de la conductividad en las medidas realizadas en atmósfera de $\mathrm{O}_{2}$ (figura 5 del artículo). El aumento de la conductividad con la presión parcial de oxígeno, es un claro indicio de conducción electrónica extrínseca, en este caso tipo p debido a la creación de huecos, tal y como se ha discutido en el punto 1.6.3 del capítulo 1.

Por lo tanto, parece que altas presiones parciales de oxígeno, son capaces de introducir la conducción tipo p en la estructura mediante la creación de huecos, sin embargo no evidencian la localización de los mismos. Tradicionalmente los huecos han sido asociados a impurezas como metales de transición, pero como se ha discutido en el capítulo 1, en estructuras dopadas con iones aceptores, parece que se localizan en los oxígenos débilmente enlazados [125,130,131].

El aumento de la conducción electrónica tipo $p$, se ve reforzada por los valores de las capacidades mostradas a bajas frecuencias, como muestra la figura 5 del artículo. Estos valores decrecen en atmósfera de $\mathrm{O}_{2}$ debido a que no ocurren en este caso transferencias de carga en la interface muestra-electrodo, asociadas a una conducción iónica.

Se puede observar una tendencia muy clara al comparar las respuestas del cambio de atmósfera en las diferentes composiciones. Existe una respuesta mayor al cambio de atmósfera cuando mayor es la cantidad de itrio incorporado, siendo YSZ7 en la que se observa un mayor cambio. Este hecho puede ser atribuible a un posible desplazamiento desde la zona más cercana al dominio electrolítico al 
dominio electrónico, más concretamente al correspondiente a la zona $p$, aumentando la cantidad de dopante presente en la estructura y en consecuencia la cantidad de iones óxido enlazados débilmente.

Otro de los objetivos a investigar es el estudio de la respuesta de las diferentes composiciones a la aplicación de un voltaje determinado. En todas las composiciones el voltaje, originó un aumento de la conductividad de la muestra cuya magnitud es dependiente de la composición. La tendencia mostrada es la misma que la que ocurre en atmósfera de oxígeno, mostrando un aumento de la conductividad mínimo (a voltajes bajos) en la muestra YSZ4, siendo mayor el efecto en la muestra YSZ7 con mayor contenido en itrio, para la aplicación del mismo voltaje (figura 7 del artículo). En la muestra YSZ7 se observa un aumento en la conductividad y un colapso del "spike" también con el voltaje, similar al producido por el cambio de atmósfera de nitrógeno a oxígeno. Este hecho parece implicar la introducción de la conducción electrónica tipo p en paralelo a la conducción iónica. En la muestra con menor cantidad de itrio, YSZ4, también se observa un aumento de la conductividad, pero sin embargo con un voltaje de $2 \mathrm{~V}$, el cambio producido es menor que en la muestra YSZ7. El mismo comportamiento es similar con el oxígeno, siendo el cambio mayor en YSZ7 en comparación con YSZ4. Estas evidencias refuerzan la hipótesis de la posibilidad de encontrarse cerca de la zona electrolítica en la muestra YSZ4 y en el dominio $p$ en la muestra YSZ7.

Las medidas realizadas con altas presiones parciales de oxígeno, permiten una quimioabsorción de oxígeno en la superficie de la muestra. Los electrones generados son utilizados para la reducción de las especies de oxígeno, tal y como indican los pasos 2, 3 y 4 del equilibrio descrito en la ecuación 3 del artículo. Posteriormente las especies de oxígeno podrán ser ionizadas generando huecos tal y como indica la ecuación 2 del artículo. 
Los iones $\mathrm{O}^{-}$resultantes constituyen la fuente de huecos y los electrones liberados actúan como fuerza de motriz para contribuir en la reducción de las especies de oxígeno del equilibrio descrito en la ecuación 3 del artículo.

Un mecanismo similar ocurre cuando se aplican a la muestra voltajes bajos. La aplicación de un voltaje $d c$ facilitará la eliminación de electrones de la muestra, aportando electrones al equilibrio descrito en la ecuación 3 del artículo, produciendo el desplazamiento a la derecha. Por lo tanto, la conductividad tipo $p$ se verá incrementada con la aplicación de pequeños voltajes.

En consecuencia, en estas composiciones ha sido posible el aumento de la conductividad tipo $p$, mediante el aumento de las presiones parciales de oxígeno o mediante la aplicación de pequeños voltajes. En ambos casos la ionización de los oxígenos débilmente enlazados es fundamental, para la formación de $\mathrm{O}^{-}$, los cuales son la principal fuente de huecos, actuando como las cargas dominantes en la conducción tipo $p$. Los electrones liberados actuaran como fuerza motriz para contribuir en la reducción de las especies oxígenos del equilibrio descrito en la ecuación 3 del artículo. Tanto el efecto de la atmósfera como el de la aplicación de pequeños voltajes, presenta una mayor sensibilidad en las muestras que contiene una mayor cantidad de itrio, siendo el efecto máximo en la muestra YSZ7 y mínimo en la YSZ4. Este comportamiento está íntimamente ligado a la cantidad de itrio, presente en la estructura, ya que permite una mayor ionización de los $\mathrm{O}^{-}$, debido a una mayor presencia de oxígenos débilmente enlazados presentando una influencia directa en los valores de conductividad del bulk y en consecuencia del material.

En el siguiente apartado se van a exponer las principales conclusiones del presente trabajo. 


\subsection{Conclusiones del artículo científico 3}

- Se han sintetizado disoluciones sólidas de la estequiometría $\mathrm{Zr}_{1-\mathrm{x}} \mathrm{Y}_{\mathrm{x}} \mathrm{O}_{2-\mathrm{x} / 2}$ (YSZ; x=0.4-0.7), mediante el método sol-gel polimérico.

- Las medidas de impedancia con diferentes atmósferas parciales de oxígeno, han demostrado que se produce un aumento de la energía de activación cuando mayor es la cantidad de itrio presente en la estructura. Este aumento se atribuye a la formación de complejos entre las vacantes de oxígeno creadas y los iones $\mathrm{Y}^{3+}$, conllevando un aumento de la energía de activación y una disminución de la conductividad iónica.

- Las medidas de impedancia con diferentes atmósferas, han demostrado la posibilidad de desplazamiento de la parte electrolítica del dominio a la parte electrónica tipo $p$, cuando mayor es la cantidad de itrio presente en la muestra. Esta tendencia es atribuida a una mayor cantidad de $\mathrm{O}^{2-}$ débilmente enlazados debido al dopaje con $\mathrm{Y}^{3+}$, y en consecuencia a la posibilidad de una mayor ionización de estos dando iones $\mathrm{O}^{-}$con la consiguiente creación de huecos que ello conlleva. Esta tendencia se pone de manifiesto al comparar las energías de activación siendo menores a presiones parciales de oxígeno altas. Esto nos indica que la energía de activación para la conducción electrónica tipo p es menor que para la conducción iónica.

- Las medidas realizadas al aplicar bajos voltajes, muestran la misma tendencia que las realizadas a altas presiones parciales de oxigeno, debido a que estos contribuyen a la ionización de los oxígenos débilmente enlazados 
en la estructura, favoreciendo la creación de huecos y por lo tanto favoreciendo la conducción electrónica tipo p.

- La aplicación de pequeños voltajes $d c$, presenta un efecto similar al del aumento de las presiones parciales de oxígeno, en todas las muestras. Viéndose este efecto incrementado en las muestras con mayor contenido en itrio.

- Se ha corroborado la existencia del ion $\mathrm{O}^{-}$. El mecanismo de creación de huecos conlleva consigo la ionización de los oxígenos débilmente enlazados. En muestras como las sintetizadas en este trabajo, de una pureza muy elevada debido al método de síntesis, la única fuente de huecos es la ionización del $\mathrm{O}^{2-}$ a $\mathrm{O}^{-}$, siendo está la fuente de huecos y por lo tanto la responsable de la conducción electrónica tipo p.

- La estequiometría $\mathrm{Zr}_{1-\mathrm{x}} \mathrm{Y}_{\mathrm{x}} \mathrm{O}_{2-\mathrm{x} / 2}$ (YSZ; $\mathrm{x}=0.4$ - 0.7), presenta la posibilidad de modular el tipo de conducción predominante en la muestra en función de la cantidad de itrio presente en la misma. Por lo tanto, el aumento de la conducción electrónica es proporcional a la cantidad de itrio presente en la disolución sólida. Esta posibilidad de modular el mecanismo de conducción hace de estos materiales muy atractivos para su uso como cátodo o como electrolito en celdas de combustible de óxido sólido (SOFC), o también como membranas cerámicas de conducción mixta iónica-electrónica, para la producción de oxígeno puro. 



\section{CAPÍTULO 6}

\section{Conclusiones generales}

"Poco conocimiento hace que las personas se sientan orgullosas. Mucho conocimiento, que se sientan humildes"

Leonardo Da Vinci 



\section{Conclusiones}

En la presente tesis doctoral se ha puesto de manifiesto la potencial multifuncionalidad de la estructura $\mathrm{Y}_{2} \mathrm{Zr}_{2} \mathrm{O}_{7}$ y su importancia en diversos campos de la ciencia. Se han abordado campos tan diferentes dentro de la ciencia, y más concretamente en el campo de la química del estado sólido, como son el de los pigmentos cerámicos, así como el de la electrocerámica. Sin embargo, la red huésped utilizada en ambos casos, ha sido el óxido el mixto de $\mathrm{ZrO}_{2}-\mathrm{YO}_{1.5}$. Este hecho, pone de manifiesto las múltiples posibilidades que confiere este sistema, siguiendo diferentes estrategias, tales como modificar sus composiciones, o bien añadir ciertas cantidades de dopante a la estructura.

De esta forma se pueden establecer las siguientes conclusiones generales:

- Se han sintetizado disoluciones sólidas con fase fluorita defectuosa basadas en $\mathrm{Y}_{2} \mathrm{Zr}_{2} \mathrm{O}_{7}$ dopado con terbio / hierro y con praseodimio / hierro mediante el método sol-gel polimérico.

- Se han sintetizados dos nuevos pigmentos cerámicos de coloración rojiza, sin el uso de elementos tóxicos actuando como cromóforos, basados en la estructura $\mathrm{Y}_{2} \mathrm{Zr}_{2} \mathrm{O}_{7}$.

- Los pigmentos han exhibido una coloración excelente en esmalte, presentado uno de ellos (el dopado con terbio y hierro) unas coordenadas cromáticas superiores al pigmento cerámico de coloración rojiza utilizado actualmente en la industria cerámica. 
- Los pigmentos han exhibido su multifuncionalidad siendo potencialmente utilizables en el campo de los denominados "cool pigments", exhibiendo unas características optimas en este campo, siendo capaces de ahorrar grandes cantidades de energía, con su aplicación en fachadas y tejados.

- Se han estudiado los mecanismos de coloración tanto en el pigmento que contenía terbio como praseodimio, jugando un papel fundamental ambos en la determinación de los estados de oxidación del hierro y por lo tanto en la coloración final del pigmento.

- El pigmento dopado con praseodimio, ha mostrado una excelente estabilidad en plástico, pudiendo ser potencialmente aplicable en el campo de los polímeros.

- Se han sintetizado disoluciones sólidas con fase fluorita defectuosa de la estequiometría $\mathrm{Zr}_{1-\mathrm{x}} \mathrm{Y}_{\mathrm{x}} \mathrm{O}_{2-\mathrm{x} / 2}(\mathrm{YSZ} ; \mathrm{x}=0.4$ - 0.7), mediante el método solgel polimérico, poniendo de manifiesto la posibilidad de estabilizar la fase fluorita defectuosa, a cantidades mayores de itrio, mediante el método solgel polimérico.

- Se han estudiado detenidamente los tipos de conducción presentes en diferentes composiciones del óxido mixto de $\mathrm{ZrO}_{2}-\mathrm{YO}_{1.5}$, y la influencia del aumento de itrio en las mismas.

- Se ha comprobado la posibilidad de aumentar la conductividad tipo $p$ en las muestras mediante dos estrategias diferentes, como el aumento de las presiones parciales de oxígeno o la aplicación de pequeños voltajes a la muestra. Ambos comportamientos corroboran la ionización de los oxígenos débilmente enlazados, siendo responsables de la ionización de los mismos a 
O-, y en consecuencia generando los huecos responsables de la conducción electrónica tipo p en ambos casos.

- Se ha demostrado la posibilidad de desplazamiento de la parte electrolítica del dominio a la parte electrónica del dominio, simplemente modificando la relación $\mathrm{Y} / \mathrm{Zr}$ presente en la estructura. Esta posibilidad de modular el mecanismo de conducción hace de estos materiales muy atractivos para su uso como cátodo o como electrolito en celdas de combustible de óxido sólido (SOFC), o también como membranas cerámicas de conducción mixta iónica-electrónica, para la producción de oxígeno puro.

En conclusión, los resultados obtenidos en la presente tesis doctoral, ponen de manifiesto la multifuncionalidad de la estequiometría $\mathrm{Y}_{2} \mathrm{Zr}_{2} \mathrm{O}_{7}$, mostrando un abanico de propiedades muy prometedoras para su potencial aplicación en campos tan diversos como son el de los pigmentos cerámicos, los materiales respetables con el medio ambiente, así como en el campo de la electrocerámica y las celdas de combustible de ion sólido. 



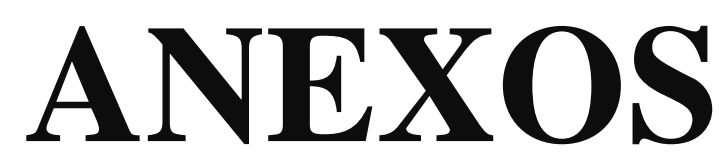

"La ciencia aún no nos ha comprobado si la locura es o no la cúspide sublime de la inteligencia"

Edgar Allan Poe 



\section{ANEXO I: TÉCNICAS DE CARACTERIZACIÓN}

“Un sutil pensamiento erróneo puede dar lugar a una indagación fructífera que revela verdades de gran valor" 



\section{A1.1. Difracción de rayos $X$, método del polvo cristalino}

La difracción de rayos X (DRX) es la técnica de caracterización más importante para dilucidar la estructura cristalina de un cierto material. Como su propio nombre indica esta técnica está basada en el fenómeno de difracción, que tiene lugar al incidir un haz de rayos $\mathrm{X}$ sobre el material cristalino. El método del polvo cristalino consiste en irradiar con un haz monocromático de rayos $\mathrm{X}$ una muestra en polvo finamente dividida, la cual presente pequeños cristales orientados al azar en cada una de las direcciones posibles. La condición de difracción en un sentido estricto, exige que las ondas dispersas por el conjunto de átomos del cristal presenten interferencias completamente constructivas, es decir que estén completamente en fase, condición que sólo se dará en determinadas direcciones concretas del espacio. Por lo tanto, para cada una de las familias de planos reticulares habrá algunos cristales orientados en el ángulo adecuado, los cuales satisfagan la ley de Bragg:

$$
n \lambda=2 d \operatorname{sen} \theta
$$

$\mathrm{n}=$ es el orden de reflexión $(\mathrm{n}=1,2,3, \ldots)$

$\lambda=$ longitud de onda de la radiación incidente

$\mathrm{d}=$ distancia interplanar

$\theta=$ angulo de Bragg

Como se observa en la figura A1.1, para que las ondas dispersadas por los distintos planos paralelos formen interferencias constructivas, la diferencia de camino recorrido por estas debe de ser múltiplo entero de la longitud de onda. 


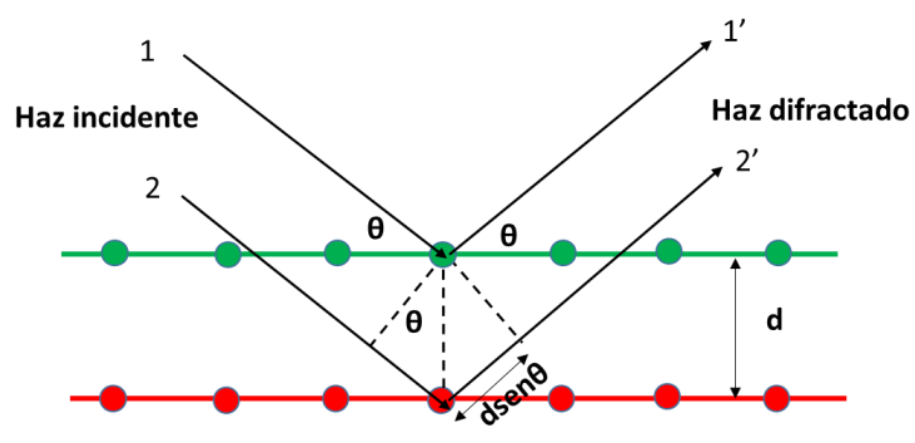

Figura A1.1. Representación esquemática de la ley de Bragg.

Por lo tanto, la diferencia de camino recorrido por el haz 2 en relación al haz 1 en su recorrido por el material, matemáticamente será 2 dsen $\theta$. Por lo tanto, para que se cumpla la ley de Bragg, n debe ser múltiplo entero de la longitud de onda.

Las direcciones de los rayos difractados vendrán determinadas por el tamaño y la forma de la red cristalina, aportando información acerca de la geometría de la celda unidad y proporcionando una medida precisa de los índices de Miller de los espaciados reticulares $(h k l)[66,124]$.

En resumen, el uso más importante del método en polvo es la identificación cualitativa de fases cristalinas o compuestos y una determinación inicial de las dimensiones y simetría de la celda unidad.

En la presente tesis los datos de difracción de rayos X, se obtienen con dos difractometros: Bruker D4 Endeavor y con Bruker D8 Advanced, ambos con radiación $\mathrm{CuK} \alpha 1$, aunque el segundo posee un monocromador primario de $\mathrm{CuK} \alpha 2$ Las condiciones de medida específicas están detalladas en la sección experimental de cada uno de los artículos. 


\section{A1.2. Microscopía electrónica de barrido (SEM) y EDX}

La microscopía electrónica de barrido es una técnica que aporta una gran información sobre la microestructura de los materiales cerámicos, pudiendo determinar la morfología de las fases presentes, el tamaño de partícula, así como identificar otras fases cristalinas no detectadas por DRX. Esta técnica se fundamenta en el estudio de las señales que emite una muestra al ser bombardeada por un haz de electrones acelerado. La información obtenida de estas señales puede ser muy variada, pudiéndose obtener información topográfica estructural y composicional. Las señales más utilizadas son de tres tipos proporcionando diferentes informaciones de la propia muestra:

- Electrones provenientes de los átomos de la muestra que han sido arrancados por el haz electrónico incidente, los denominados electrones secundarios. En una interacción inelástica, el electrón incidente transfiere parte de su energía a un electrón externo del átomo, la suficiente como para que sea capaz de salir expulsado del átomo. Los electrones secundarios provienen de una zona de la muestra muy poco profunda de alrededor de los 50-500 Å. En este sentido, la información obtenida es fundamentalmente topográfica, producida por la morfología de la superficie de la muestra.

- Electrones provenientes del propio haz incidente, que han rebotado con los núcleos de los átomos que componen la muestra, denominados electrones retrodispersados. En una interacción elástica, el conjunto del sistema que sufre la colisión mantiene constante el momento lineal y la energía cinética. En la descripción del proceso en microscopía electrónica se supone que el electrón incidente sufre una gran desviación angular de su trayectoria, manteniendo su 
energía cinética prácticamente invariable. Esta interacción elástica tiene lugar generalmente entre el electrón incidente y el núcleo atómico. Los electrones son dispersados con un ángulo superior a noventa grados. Estos electrones provienen de una zona mucho más profunda que los secundarios.

- Rayos X característicos de los elementos que componen la muestra, cuya generación ha sido explicada en el anexo A1.1. Básicamente, el electrón incidente suministra la energía necesaria para excitar el átomo, de forma que parte de su energía o su totalidad es empleada para expulsar un electrón de las capas internas del átomo, que queda excitado en un nivel superior de energía. Para retornar a su nivel fundamental de energía, un electrón de las capas externas va a ocupar el hueco dejado en la capa interna, emitiendo una radiación de energía en forma de fotón de rayos $\mathrm{X}$, característica del propio elemento. Estos permiten la realización del análisis elemental de la muestra. Este microanálisis o análisis por energías dispersivas de rayos X (EDX), permite realizar análisis semicuantitativos de las muestras preparadas [66].

En la presente tesis, el estudio microestructural de las muestras se ha realizado en un microscopio electrónico de barrido de JEOL, modelo $7001 \mathrm{~F}$, equipado con un voltaje de aceleración máximo de $30 \mathrm{Kv}$. Las condiciones de medida están detalladas en la sección experimental de cada artículo. 


\section{A1.3. Espectroscopia ultravioleta-visible- infrarrojo cercano.}

La espectroscopia ultravioleta-visible, está basada en el proceso de absorción de la radiación ultravioleta-visible (radiación con longitud de onda comprendida entre 200 y 780 nm aproximadamente) por una molécula o un material. La absorción de esta radiación causa la promoción de un electrón a un estado excitado. Estas transiciones electrónicas que se producen permiten evaluar la estructura y propiedades de la materia. En general, se consideran dos tipos de transiciones posibles en sólidos:

- Transiciones electrónicas de electrones $d$ y $f(d-d, f-f)$ en iones de metales de transición, lantánidos y actínidos.

- Transferencias de carga entre el metal y el ligando.

Estas transiciones son de baja energía, por lo tanto, aparecen habitualmente en la región visible del espectro, lo que explica el desarrollo del color en sólidos que presentan metales de transición $(d-d)$ [185].

La espectroscopia a longitudes de onda mayores a $800 \mathrm{~nm}$, corresponde a región del infrarrojo cercano (NIR). En esta zona del espectro electromagnético el proceso de absorción es debido a presencia de sobretonos y bandas originadas por transiciones vibracionales intensas que se producen a longitudes de onda mayores $[66,185]$.

En la presente tesis el estudio por espectroscopia ultravioleta-visibleinfrarrojo cercano (UV-VIS-NIR), se realizó a temperatura ambiente mediante un espectrofotómetro Ultravioleta-Visible IR Cary Varian Cary 500. Los espectros se registraron haciendo uso de una esfera integradora. Las condiciones de medida están detalladas en la sección experimental de cada artículo. 


\section{A1.4. Colorimetría}

La observación de un color u otro en un material, se debe a la absorción de una radiación con una determinada longitud de onda característica, y a su vez al reflejo de la longitud de onda del color complementario.

Existen varios sistemas con el objetivo de medir de una forma objetiva las características del color. El sistema más ampliamente empleado en el campo de la cerámica es el método denominado CIE-L*a*b*. Este sistema se representa tal y como se indica en la figura A1.2, como un sistema coordenado cartesiano definido por tres coordenadas colorimétricas: $\mathrm{L}^{*}, \mathrm{a}^{*} \mathrm{y} \mathrm{b}^{*}$. La coordenada $\mathrm{L}^{*}$ se refiere a la intensidad o luminosidad del color adoptando valores en el intervalo desde $L^{*}=100$ (blanco) hasta $\mathrm{L}^{*}=0$ (negro). Las coordenadas $\mathrm{a}^{*} \mathrm{y} \mathrm{b}^{*}$ se refieren a las características cromáticas o tono, siendo la coordenada $\mathrm{a}^{*}>0$ la correspondiente a la tonalidad roja, y la $\mathrm{a}^{*}<0$ a la verde. Análogamente, la coordenada $\mathrm{b}^{*}>0$ se refiere a la tonalidad amarilla y la $\mathrm{b} *<0$ a la azul $[66,74]$.

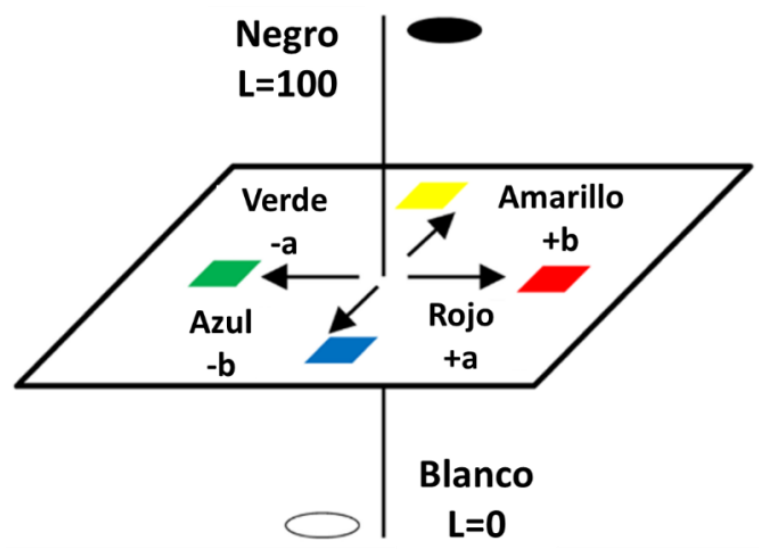

Figura A1.2. Representación espacial del sistema CIE-L*a*b*.(Adaptación de la referencia [186].) 
En la presente tesis, el análisis del color se evaluó de acuerdo con las especificaciones establecidas por la CIE (Comission Internacionale de l’Eclairage) a partir de los parámetros L*a*b*. El iluminante utilizado fue el D65, el cual representa la luz diurna media con excitación ultravioleta, utilizando el espectrofotómetro Ultravioleta-Visible Cary Varian Cary 500. Las coordenadas cromáticas fueron determinadas en un intervalo de longitudes de onda de 380 a 700 nm usando un blanco de $\mathrm{BaSO}_{4}$.

\section{A1.5. Espectroscopia de fluorescencia}

La energía de un estado excitado electrónicamente se puede perder de varias formas. Una de ellas es la perdida mediante mecanismos de vibración, rotación y translación, convirtiendo esta energía de excitación en calor. Sin embargo, existen otras posibilidades, como que una molécula tome parte en una reacción fotoquímica o la desintegración radiactiva que tiene lugar cuando una especie química despendre su energía de excitación en forma de fotón. Se conocen dos mecanismos de desintegración radiactiva, la fluorescencia y la fosforescencia. En el primero la radiación emitida cesa casi inmediatamente al extinguirse la radiación de excitación. En cambio en la fosforescencia la emisión puede persistir durante periodos largos de tiempo.

Dentro de la fluorescencia, existen dos tipos de fluorescencia. Cuando la frecuencia de resonancia en la cual la radiación absorbida es remitida sin cambio de la frecuencia; y la más habitual, donde las bandas de fluorescencia se encuentran centradas a longitudes de onda más largas que la línea de resonancia. Este fenómeno se denomina desplazamiento de Stokes.

Cuando uno de los electrones de una especie química es excitado a un nivel superior, da lugar a un estado excitado singlete o singulete (espines apareados) o a 
un estado triplete (espines desapareados). En el estado excitado singlete, el espín del electrón promocionado está todavía apareado con el electrón del estado fundamental; sin embargo, en el estado excitado triplete los espines de los dos electrones se han desapareado y están por tanto paralelos.

Los fenómenos de fluorescencia implican especies químicas que absorben radiación ultravioleta o visible de la frecuencia adecuada a uno o más de los electrones apareados (habitualmente electrones $\pi$ en el caso de moléculas orgánicas no saturadas) para a un estado excitado singlete, en el que el espín del electrón no ha variado y por tanto el espín neto sigue siendo nulo. La transición directa desde el estado fundamental singlete hasta el estado excitado triplete es una transición prohibida (muy poco probable) y por tanto, la única manera de poblar el estado excitado triplete es convirtiendo un estado excitado singlete en un estado excitado triplete.

La figura A1.3 muestra el diagrama parcial de energía para un sistema fotoluminiscente.

Las dos líneas gruesas de la izquierda representan los estados electrónicos excitados singlete primero $\left(\mathrm{S}_{1}\right)$ y segundo $\left(\mathrm{S}_{2}\right)$. El de la derecha $\left(\mathrm{T}_{1}\right)$ representa la energía del primer estado electrónico excitado.

Como muestra la figura A1.3, la excitación de puede tener lugar por absorción de dos bandas de radiación, una centrada alrededor de la longitud de onda $\lambda_{1}\left(\mathrm{~S}_{0} \rightarrow \mathrm{S}_{1}\right)$ y la segunda centrada alrededor de la longitud de onda más corta $\lambda_{2}\left(\mathrm{~S}_{0} \rightarrow \mathrm{S}_{2}\right)$. La excitación lleva a la especie a cualquiera de los diversos estados vibracionales $[187,188]$. 


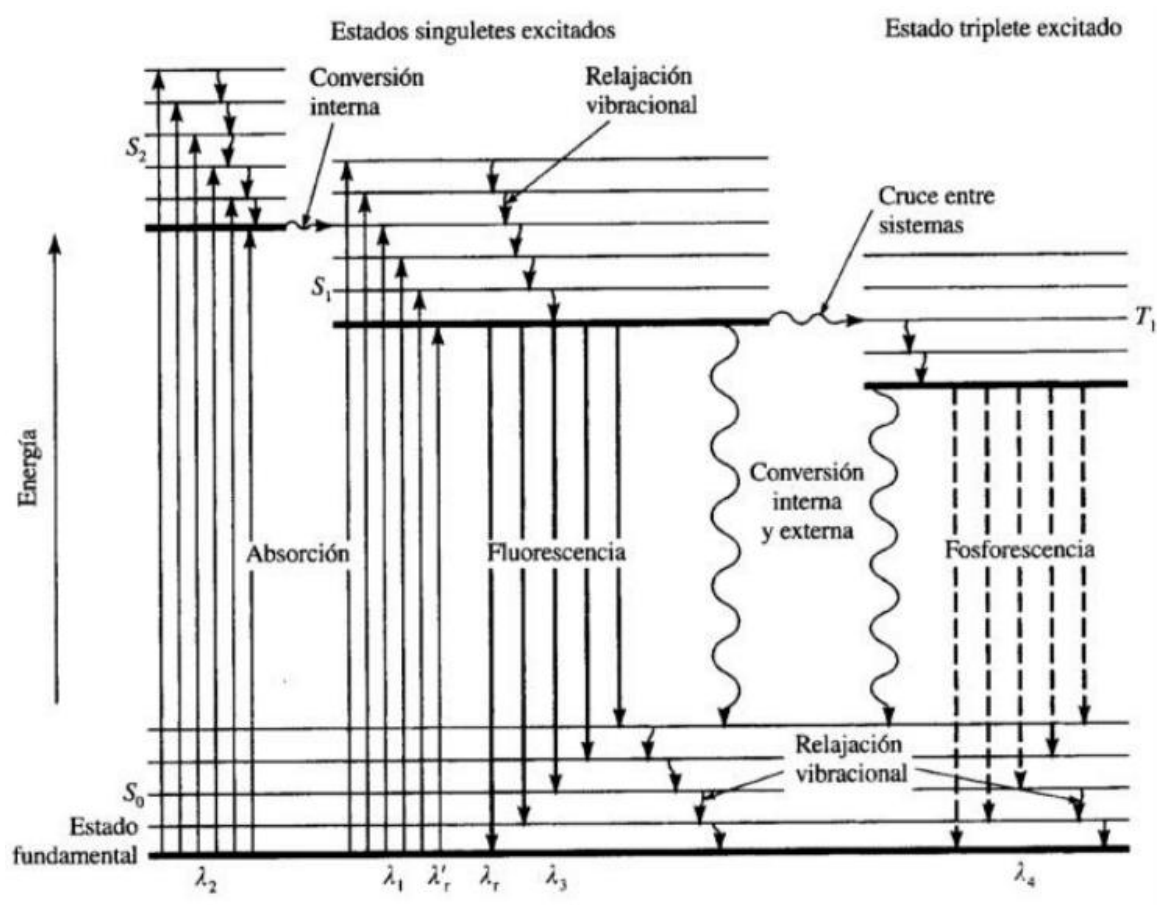

Figura A1.3. Diagrama parcial de energía para un sistema fotolumiscente [188].

Una especie puede volver a su estado fundamental por una combinación de varias etapas. Como se observa en la figura A1.3 dos de estas etapas la fluorescencia y la fosforescencia implican la emisión de un fotón de radiación. Las otras etapas de desactivación o relajación, están indicadas por las flechas onduladas y son procesos que no implican una radiación. El camino más probable hacia el estado fundamental es aquel que minimiza el tiempo de vida del estado excitado. Por tanto, si la desactivación por fluorescencia es rápida con respecto a los procesos sin radiación, se observa tal emisión. Por lo contrario si un camino sin radiación es más favorable cinéticamente, la fluorescencia no tendrá lugar o será menos intensa.

Con el objetivo de maximizar la población de especies que pueden alcanzar el estado excitado, previamente a la excitación de estos se debe de realizar un espectro de excitación. Los espectros de excitación se obtienen midiendo la intensidad luminiscente a una longitud de onda fija (generalmente la 
correspondiente al máximo de emisión fluorescente) mientras se varia la longitud de onda de excitación. En cambio, las medidas de los espectros de emisión se realizan mediante la excitación a una longitud de onda determinada, donde la absorción es máxima ( $\lambda$ excitación), mientras se registra la intensidad de emisión en función de la longitud de onda [187] .

En la presente tesis, las medidas de fotoluminiscencia se realizaron en un espectrofotómetro de fluorescencia Varian Cary Eclipse. Las condiciones de medida están detalladas en la sección experimental de cada artículo.

\section{A1.6. Espectroscopia Fotoelectrónica de Rayos X (XPS)}

La espectroscopia fotoelectrónica de rayos X (XPS) es una técnica analítica que mide las energías de los fotoelectrones que son emitidos por los átomos cuando estos son irradiados por fotones de rayos $X$ con baja energía (1-2KeV). Es una técnica altamente específica para superficies debido al corto rango de fotoelectrones que son excitados en el sólido. La energía de los fotoelectrones que abandonan la superficie de la muestra origina un espectro proporcionando información sobre la energía de cada nivel y por tanto, sobre la naturaleza de cada átomo emisor.

El análisis por XPS de una superficie puede proporcionar información tanto cualitativa y cuantitativa de todos los elementos presentes, excepto $\mathrm{H}$ y He. Las principales aplicaciones de esta técnica son:

- Identificación de todos los elementos presentes (excepto H y He) en concentraciones mayores al $0.1 \%$. 
- Determinación semicuantitativa de la composición elemental de la superficie.

- Información acerca del entorno molecular: estado de oxidación átomos enlazantes, orbitales moleculares, etc.

Para conocer la técnica XPS, previamente se ha de comprender el efecto fotoeléctrico y de fotoemisión. Cuando un fotón se encuentra con un átomo puede ocurrir:

- Que pueda atravesarlo sin interacción alguna.

- Que sea dispersado por un electrón de un orbital atómico con lo que ocurre una pérdida de energía.

- Que el fotón interaccione con un electrón de un orbital atómico con una transferencia total de la energía del fotón hacia el electrón, ocurriendo la emisión del electrón del átomo.

El tercer proceso resulta básico para la técnica XPS.

Cuando ningún electrón ha sido emitido por el átomo, se debe a que la frecuencia de excitación del fotón es demasiado baja. Cuando aumentamos gradualmente la energía del fotón se comienza a observar la fotoemisión de electrones del átomo. Una vez superada esta frecuencia umbral, el número de electrones emitidos será proporcional a la intensidad de iluminación (mayor número de fotones de alta frecuencia de excitación). Además, la energía cinética de los electrones emitidos es linealmente proporcional a la frecuencia de los fotones excitantes, si se utilizan fotones de energía muy superior a la umbral, el exceso de energía es transmitido al electrón que se emite. Este proceso de fotoemisión es extremadamente rápido y su física básica se describe mediante la ecuación de Einstein:

$$
\mathrm{E}_{\mathrm{B}}=h v-\mathrm{EK}
$$


Donde $\mathrm{E}_{\mathrm{b}}$ es la energía de enlace del electrón en el átomo, $\mathrm{h} v$ es la energía de la fuente de rayos X, y EK es la energía cinética del electrón detectado que es medida por el espectrómetro del XPS.

Por lo tanto un electrón cargado negativamente se unirá al átomo por atracción con su núcleo positivo. Cuanto más interno es el electrón, más fuerte será su enlace. Esta energía variara según el tipo de átomo y de los átomos que se unan a él. La energía de enlace que se mide en XPS se asocia a enlaces tipo iónico o covalente entre átomos, siendo las interacciones débiles entre átomos, tales como los enlaces de hidrogeno despreciables.

Por lo tanto la energía de enlace de un fotoelectrón emitido es simplemente la diferencia de energía entre el electrón $n-1$ del estado final y el electrón n del estado inicial de energía:

$$
\mathrm{E}_{\mathrm{B}}=\mathrm{E}_{\mathrm{F}}(n-1)-\mathrm{E}_{\mathrm{i}}(n)
$$

Si durante el proceso de fotoemisión no ocurre un reordenamiento de electrones en el átomo, entonces la energía de enlace será igual al valor negativo de la energía orbital, $-\varepsilon_{\mathrm{k}}$, para el electrón fotoemitido.

$$
\mathrm{E}_{\mathrm{B}} \approx-\varepsilon_{\mathrm{k}}
$$

Pero durante el proceso de fotoemisión los otros electrones de la muestran no permanecen imperturbables, sino que pueden responder a la creación del hueco electrónico mediante un reordenamiento de las capas internas, o minimizando la energía del átomo ionizado. La reducción de energía que se produce de esta manera se conoce como energía de relajación, y ocurre tanto para electrones del átomo que contiene el hueco electrónico (relajación atómica), como para electrones de átomos vecinos (relajación extra-atómica). De este modo la energía de enlace queda redefinida como: 


$$
\mathrm{E}_{\mathrm{B}} \approx-\varepsilon_{\mathrm{k}}-\mathrm{E}_{\mathrm{r}}(\mathrm{k})
$$

El estado inicial de energía es el estado fundamental de energía del átomo, antes de ser sometido a los procesos de fotoemisión. Los efectos de relajación pueden tener una importancia significativa en la energía de enlace medida. En todos los casos, el reordenamiento de electrones que ocurre durante el proceso de

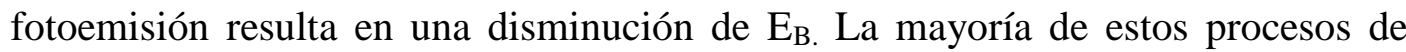
relajación derivan del reordenamiento de los electrones de las capas más externas, los cuales poseen una $E_{B}$ menor que la del fotoelectrón emitido.

Además también existen otros tipos de efectos sobre el estado final de energía y que contribuyen a la $\mathrm{E}_{\mathrm{B}}$. Estos son el desdoblamiento en multipletes y la aparición de picos satélites. El primero se origina por la interacción del hueco electrónico con electrones desapareados de orbitales más externos, mientras que el segundo surge cuando el fotoelectrón emitido pierde parte de su energía cinética para excitar a un electrón de valencia hacia un orbital desocupado (principalmente transiciones $\left.\pi-\pi^{*}\right)$.

Dado que la $\mathrm{E}_{\mathrm{B}}$ se determina midiendo la energía cinética del fotoelectrón emitido, se requiere una energía de enlace de referencia. Para ello se utilizan metales conductores que son colocados en contacto con el espectrómetro, como el cobre o el oro [187].

En la presente tesis los datos de espectroscopia fotoelectrónica de rayos $\mathrm{X}$ (XPS) se obtienen a partir de dos equipos: un espectrómetro SPECS equipado con un detector 150-MCD-9 y un X-Alpha Thermo Scinetific. Ambos utilizaron como fuente de rayos $\mathrm{X}$ un radiación $\mathrm{AlK} \alpha(1486.6 \mathrm{eV})$. Las condiciones de medida específicas, están detalladas en la sección experimental de cada artículo. 


\section{A1.7. Espectroscopia de impedancia}

La espectroscopia de impedancia (IS) es una técnica ampliamente utilizada para la caracterización y el estudio de las propiedades eléctricas de numerosos materiales, al igual que sus interfases. Uno de sus principales usos es en el estudio de electrocerámicas. Esta técnica, utiliza el comportamiento diferente con la frecuencia de cada uno de los constituyentes de una cerámica, permitiendo distinguir entre diferentes regiones de la misma. Para ello se mide la impedancia de una muestra en un intervalo amplio de frecuencias, por ejemplo de $10^{-2}$ a $10^{-7} \mathrm{~Hz}$, aplicando a través de la misma una corriente alterna. La corriente medida se puede analizar como una suma de funciones sinusoidales, asumiendo una respuesta lineal. La corriente producida por la aplicación del voltaje sinusoidal, es sinusoidal a la misma frecuencia pero se encuentra fuera de fase, debido al efecto inductivo. Como muestra la figura A1.4.

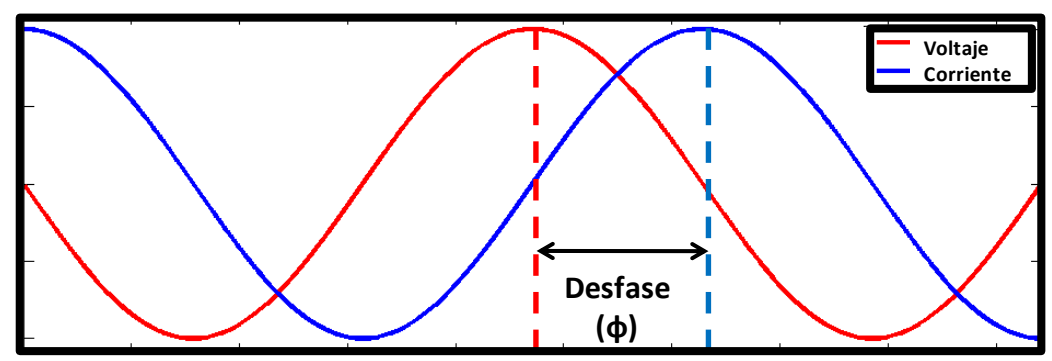

Figura A1.4. Representación de la respuesta de la corriente en un sistema lineal.

La señal de la aplicación del voltaje, expresada en función del tiempo, tiene la forma:

$$
E_{t}=E_{0} \sin (\omega t)
$$


Siendo $E_{t}$ el voltaje a un tiempo $t, E_{0}$ la amplitud de la señal y $\omega$ la frecuencia angular. La relación entre la frecuencia angular $\omega$ (radianes/segundo) y la frecuencia $f$ (Hertz) es:

$$
\omega=2 \pi f
$$

Debido al desfase entre la señal de excitación y la señal de respuesta, está tiene la siguiente forma:

$$
I_{t}=I_{0} \sin (\omega t+\phi)
$$

De este modo de una forma análoga a la ley de ohm, se puede calcular la impedancia del sistema como:

$$
R=\frac{V}{I}=Z=\frac{E_{t}}{I_{t}}=\frac{E_{0} \sin (\omega t)}{I_{0} \sin (\omega t+\phi)}=Z_{0} \frac{\sin (\omega t}{\sin (\omega t+\phi)}
$$

Por lo tanto se puede expresar la impedancia en términos de $\mathrm{Z}_{0} \mathrm{y}$ de un desfase $\phi$.

Mediante la relación de Euler,

$$
\exp (j \phi)=\cos \phi+\mathrm{j} \sin \phi
$$


$(j=\sqrt{-1})$ pudiendose expresar la impedancia como una función compleja. Por lo tanto el voltaje se puede describir como:

$$
E_{t}=E_{0} \exp (j \omega t)
$$

y la corriente como:

$$
I_{t}=I_{0} \exp (j \omega t+\phi)
$$

De este modo a partir de la ecuación A1.6, se puede representar la impedancia como:

$$
Z(\omega)=\frac{E}{I}=Z_{0} \exp (j \phi)=Z_{0}(\cos \phi+\mathrm{j} \sin \phi)=\mathrm{Z}^{\prime}+\mathrm{j} \mathrm{Z}^{\prime \prime}
$$

\section{Representación de los datos}

Para la representar los datos de impedancia, el sistema más habitual es mediante diagramas de Nyquist, donde se representa la parte imaginaria Z" (capacitiva) frente a la parte real Z' (resistiva). Representando la parte imaginaria (eje de ordenadas) frente a la parte real (eje de abscisas) se obtiene la representación del plano complejo de la impedancia, tal y como se muestra en la figura A1.5. Cuando un sistema se encuentra en un estado estacionario y es perturbado, por dicha corriente alterna, está tendera de nuevo a relajarse a su estado estacionario. 


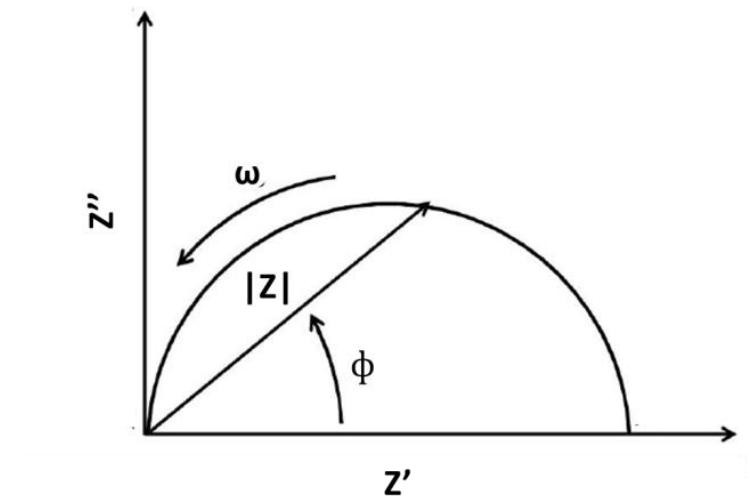

Figura A1.5. Representación del plano complejo de la impedancia (Adaptación de la referencia [189].)

Este tiempo necesario para que tenga lugar dicha relajación se denomina constante de tiempo $(\tau)$, y es característico de las diferentes regiones de una muestra. Los procesos rápidos, es decir con $\tau$ bajas, tienen lugar a altas frecuencias, en cambio los procesos lentos, tienen lugar a frecuencias bajas. Cada proceso se relaciona con una frecuencia característica.

$$
f=\frac{1}{2 \pi \tau}
$$

Las medidas de impedancia incluyen tanto un componente resistivo como otro reactivo (capacitancia / inductancia). Las diferentes regiones de una cerámica son caracterizadas por una resistencia y una capacitancia normalmente localizadas en paralelo. El tiempo de relajación cada elemento RC en paralelo viene dado por el producto de $\mathrm{R} \mathrm{y} \mathrm{C}$.

$$
\tau=R C
$$

A partir de la ecuación A1.14 y A1.15 y conociendo que la relación entre la frecuencia angular $\omega$ (radianes/ segundo) y la frecuencia $f(\mathrm{~Hz})$ es $\omega=2 \pi f$ se puede extraer el valor de frecuencia de perdida máxima en el espectro de impedancias, como muestra la siguiente ecuación.

$$
\omega_{\max } R C=1
$$


Por lo tanto, a través de un espectro de impedancia se podrán diferenciar diferentes elementos RC pudiendo asociar cada uno de ellos a una región característica de la muestra. Cada elemento RC da lugar a un semicírculo (idealmente) a partir del cual se pueden obtener los valores de R y C. Estos elementos RC en serie, están separados debido a que el máximo del semicírculo se cumple la ecuación A1.16.

Por lo tanto, los valores de resistencia de cada elemento se obtiene en el punto de corte sobre el eje Z', en cambio los valores de capacidad se obtienen aplicando la ecuación A1.16 a la frecuencia del máximo del semicírculo. Un ejemplo de ello se muestra en la figura A1.6.

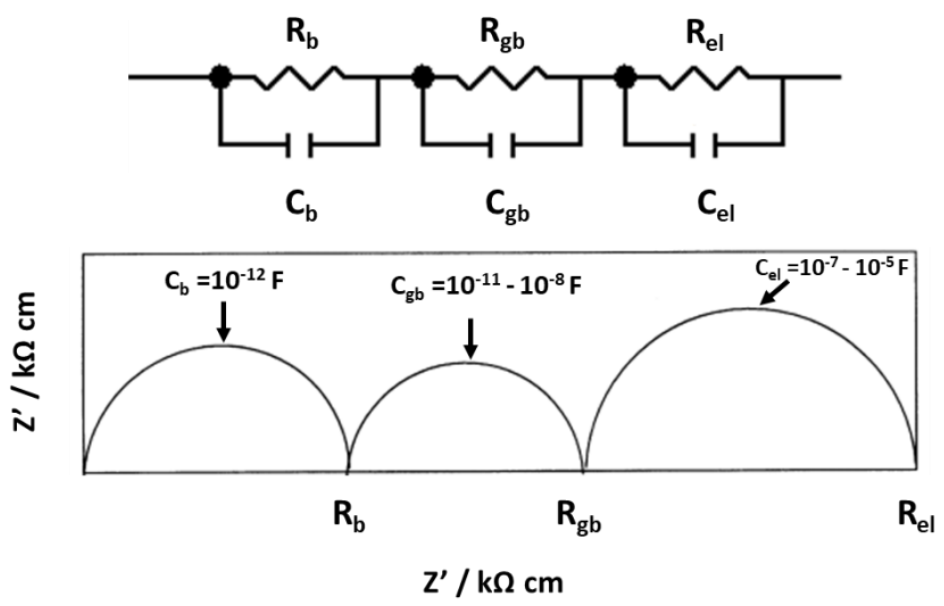

Figura A1.6. Datos de impedancia representados en forma de plano complejo de la impedancia, Z" frente a Z'. El circuito equivalente utilizado para interpretar los datos se muestra en el margen superior. Donde $\mathrm{R}_{\mathrm{b}} \mathrm{y} \mathrm{C}_{\mathrm{b}}$ se refieren a la resistencia y la capacidad asociada al bulk respectivamente; $\mathrm{R}_{\mathrm{gb}} \mathrm{y} \mathrm{C}_{\mathrm{gb}}$ a las de las fronteras de grano y $\mathrm{R}_{\mathrm{el}} \mathrm{y} \mathrm{C}_{\mathrm{el}}$ a la interfase muestra electrodo.

Una vez obtenidos estos valores, se pueden asociar a diferentes regiones de la muestra. Para ello se utiliza los valores de las capacidades mostrados en la siguiente tabla: 
Tabla A1.1. Valores de capacidad y su posible interpretación [190].

\begin{tabular}{cc}
\hline Capacidad/ F & Fenómeno responsable \\
\hline $\mathbf{1 0}^{-12}$ & Interior de grano (bulk) \\
$\mathbf{1 0}^{-11}$ & Segundas fases minoritarias \\
$\mathbf{1 0}^{-11}-\mathbf{1 0}^{-8}$ & Borde de grano \\
$\mathbf{1 0}^{-10}-\mathbf{1 0}^{-9}$ & Bulk en ferroeléctricos \\
$\mathbf{1 0}^{-9}-\mathbf{1 0}^{-7}$ & Capas superficiales \\
$\mathbf{1 0}^{-7}-\mathbf{1 0}^{-5}$ & Interfase electrodo-muestra \\
$\mathbf{1 0}^{-4}$ & Reacciones electroquímicas \\
\hline
\end{tabular}

Con el objetivo de entender los diferentes componentes existentes en una muestra cerámica caracterizada por espectroscopia de impedancia se muestra la figura A1.7.

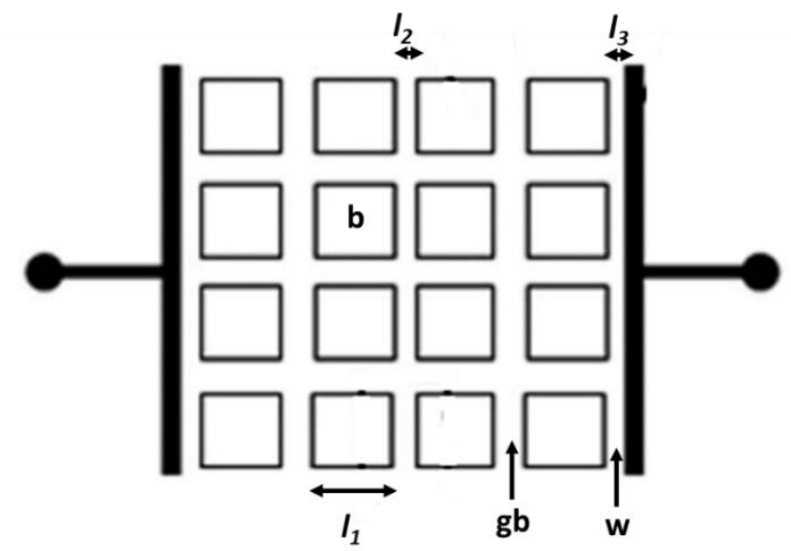

Figura A1.7. Modelo de ladrillos (brickwork model). Interior de grano (b), límite de grano (gb) y interfase electrodo muestra (w). (Adaptación de la referencia [190].)

En esta figura se describe una muestra cerámica mediante el modelo de ladrillos (brick model). En ella se observa una cerámica compuesta por el interior de grano (bulk) de una dimensión $\mathrm{l}_{1}$, separados entre ellos por los límites de grano de espesor $\mathrm{l}_{2}$. El material se encuentra confinado entre dos capas de un material 
conductor (normalmente oro o platino) actuando como conductor, de un espesor $l_{3}$, como se muestran en la figura. De esta forma podemos considerar una muestra de impedancia, como un condensador de láminas paralelas, con un área A y una separación entre las placas l (los electrodos).

\section{Procesado de datos}

Adicionalmente a la representación del plano complejo de la impedancia Z" frente a Z', para la interpretación de resultados existen otros formalismos alternativos capaces de proporcionar información adicional, difícilmente extraíble del plano complejo debido a la complejidad de la muestra. En muchas muestras no se van a observar necesariamente los elementos de la muestra (borde de grano y bulk) bien definidos, debido a que en muchos casos uno de los elementos presenta una resistencia mucho mayor que otro, por lo tanto, el elemento con mayor resistencia presentara un semicírculo mucho mayor, enmascarando al otro componente, observándose solamente un único semicírculo, o un semicírculo con una ligera distorsión, tal y como se observa en la figura A1.8a.

Con el objetivo de poder estudiar por separado la respuesta asociada al límite de grano y del interior del grano, los datos de impedancia se procesan y se representan en forma de gráfica espectroscópica de los componentes imaginarios Z" y M", tal y como se muestra en la figura A1.8b.

La representación espectroscópica de los componentes imaginarios Z' y M" se compone de dos graficas Z" y M" frente a la frecuencia, representadas ambas conjuntamente en una misma gráfica común, ya que:

$$
M^{*}=j \omega C_{0} Z^{*}=M^{\prime}+j M^{\prime \prime}
$$



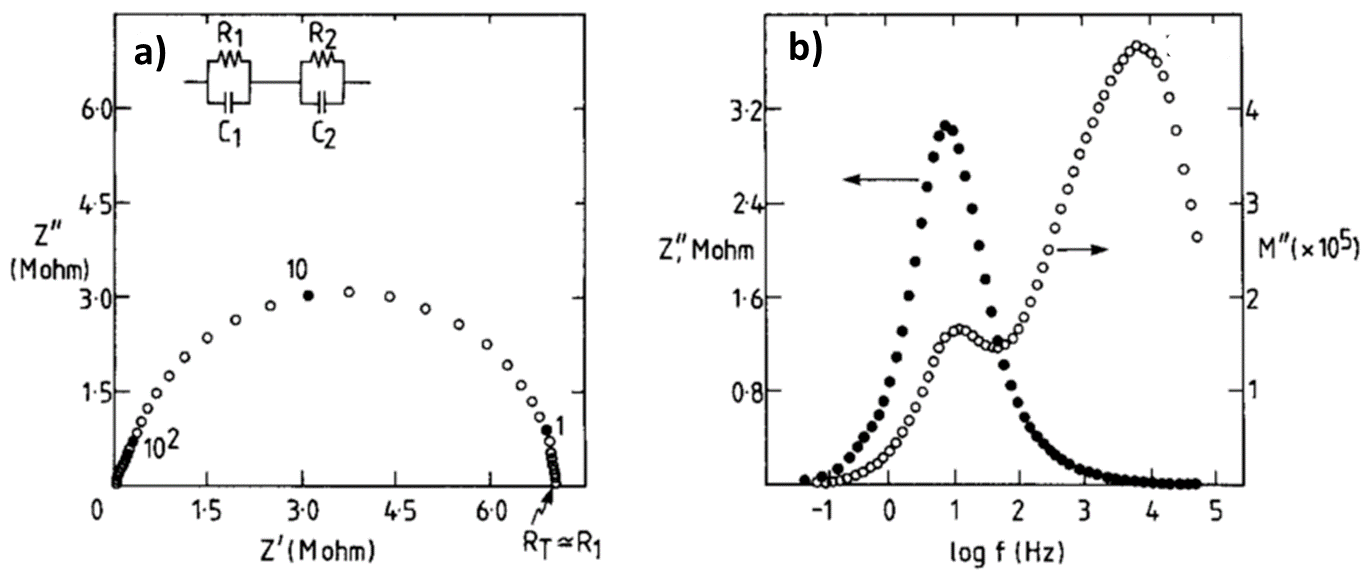

Figura A1.8. Datos de impedancia representados en formato de plano complejo de la impedancia (a) y de graficas espectroscópicas de los componentes imaginarios M' y Z' (b). El circuito equivalente utilizado para interpretar los datos se muestra en el margen superior izquierdo de la figura (a) (Adaptación de la referencia [190].)

Donde $j=\sqrt{-1}, \omega=2 \pi f$ (frecuencia angular), $C_{0}$ es la capacidad en el vacío, $M^{\prime}=j \omega C_{0} Z^{\prime \prime}$ y $M^{\prime \prime}=\omega C_{0} Z^{\prime}$, la representación espectroscópica de $Z$ "' y M"' se puede considerar como la representación de la parte real y imaginaria de la impedancia, $\mathrm{Z}^{*}$, frente a la frecuencia.

Por lo tanto, a partir de la gráfica de impedancia Z" se destacan los elementos más resistivos (borde de grano), en cambio mediante el modulo, M", destaca aquellos con menor capacidad (interior de grano).

En la figura A1.8a, se observa un único semicírculo con una ligera desviación de la idealidad en la parte de altas frecuencias. En la figura A1.8b en la gráfica espectroscópica de Z" se observa un único pico, correspondiente al semicírculo único observado en $Z^{*}$, en cambio en la gráfica de M' se observan dos picos. Esto es debido a que en $M$ "' se observa tanto la respuesta eléctrica del interior de grano (alta frecuencia) como la del borde de grano (bajas frecuencias). 
A partir de las gráficas espectroscopias de Z" y M" (figura A1.8b), se pueden obtener los valores de C y R asociados a cada componente, considerando que en un circuito RC en paralelo la impedancia total es la suma de las inversas de la impedancia de la resistencia $\left(Z_{R}=R\right)$ y de la impedancia del condensador $\left(Z_{C}=1 / j \omega C\right)$ :

$$
\frac{1}{Z_{T}}=\frac{1}{Z_{R}}+\frac{1}{Z_{C}}=\frac{1}{R}+j \omega C=\frac{R}{1+j \omega R C}
$$

Donde la parte real y la imaginaria de $Z^{*}\left(Z^{*}=Z^{\prime}+j Z^{\prime}{ }^{\prime}\right)$ son:

$$
\begin{gathered}
Z^{\prime \prime}=-R\left[\frac{\omega R C}{1+(\omega R C)^{2}}\right] \\
Z^{\prime}=\frac{R}{1+(\omega R C)^{2}}
\end{gathered}
$$

Teniendo en cuenta la ecuación A1.18 se obtiene la parte real y imaginaria de M'?

$$
\begin{aligned}
& M^{\prime}=\frac{C_{0}}{C}\left[\frac{(\omega R C)^{2}}{1+(\omega R C)^{2}}\right] \\
& M^{\prime \prime}=\frac{C_{0}}{C}\left[\frac{(\omega R C)}{1+(\omega R C)^{2}}\right]
\end{aligned}
$$

Teniendo en cuenta la ecuación a A1.16 y sustituyéndola en las ecuaciones A1.19 y A1.22 se obtiene la relación entre los valores de Z" ${ }_{\text {max }}$ y la resistencia, y los valores de M" ${ }_{\max } \mathrm{y}$ de capacidad. 


$$
\begin{gathered}
Z_{\text {max }}^{\prime \prime}=\frac{R}{2} \\
M_{\text {max }}^{\prime \prime}=\frac{C_{0}}{2 C}
\end{gathered}
$$

Siendo el valor de $\mathrm{C}_{0}=\varepsilon_{0}$ (permitividad del vacío, $8.854 \times 10^{-14} \mathrm{~F} \mathrm{~cm}^{-1}$ ). 



\section{ANEXO II: NOTACIÓN DE KRÖGER- VINK}

"Lo más incomprensible acerca de este mundo es que es comprensible" Albert Einstein 



\section{A2.1. Notación de Kröger-Vink}

En los materiales iónicos, la conducción ocurre mediante el movimiento difusivo de iones entre posiciones vacantes a altas temperaturas. Por lo tanto, la conducción iónica requiere de imperfecciones y vacantes en la estructura para que se produzca conducción. En este sentido, la notación Kroger-Vink [115] es la notación estándar utilizada en la descripción de defectos en materiales iónicos. Se esquematiza de la siguiente forma.

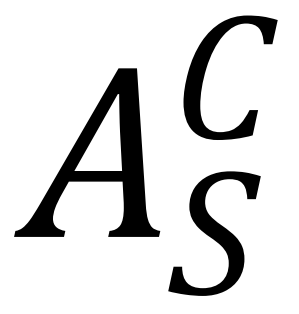

S: símbolo químico de la especie que debería ocupara la posición estructural.

A: símbolo químico de la especie o $\mathrm{V}$ si es una vacante que está ocupando la posición estructural del elemento S.

C: carga efectiva (o carga relativa) representada por $(\cdot)$ si es positiva, por (') si es negativa y por (x) si hay electroneutralidad.

El cristal perfecto se representa por nil, los electrones por $e$ y los huecos por $h$. En la siguiente tabla se enumeran los defectos más relevantes empleados en la presente tesis.

Tabla A2.1. Defectos puntuales más empleados en el desarrollo de esta tesis.

\begin{tabular}{cc}
\hline Tipo de defecto & Símbolo \\
\hline Vacante de oxígeno & $V_{o}^{*}$ \\
Itrio en la posición del $\mathrm{Zr}$ & $Y_{\dot{Z}_{r}}$ \\
Oxígeno en su posición estructural & $\mathrm{O}_{\mathrm{o}}^{\mathrm{x}}$ \\
electrón & $e^{\prime}$ \\
Hueco electrónico & $h$ \\
\hline
\end{tabular}





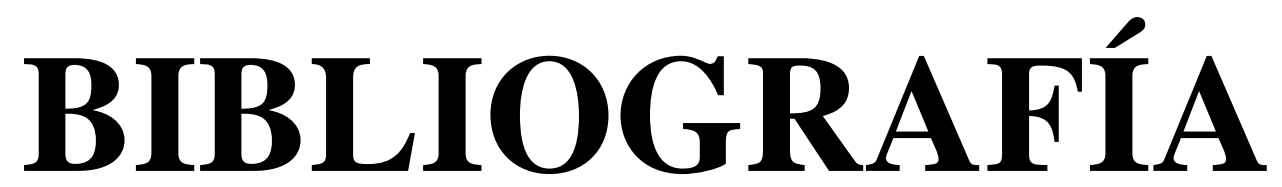

"Cuando bebas agua, recuerda la fuente"

Proberbio Chino 



\section{BIBLIOGRAFÍA}

[1] J. Wu, X. Wei, N.P. Padture, P.G. Klemens, M. Gell, P. Miranzo, M.I. Osendi, J. Am. Cer. Soc 35, 3031-3035 (2002).

[2] R. Vassen, X. Cao, F. Tietz, D. Basu, D. Stöver, J. Am. Ceram. Soc. 83, 2023-2028 (2000).

[3] N.P. Padture, M. Gell, E.H. Jordan, Science 296, 280-284 (2002).

[4] B.P. Mandal, S.K. Deshpande, A.K. Tyagi, J. Mater. Res. 23, 911-916 (2008).

[5] B. Liu, J.Y. Wang, Y.C. Zhou, T. Liao, F.Z. Li, Acta Mater. 55, 2949-2957 (2007).

[6] H. Lehmann, D. Pitzer, G. Pracht, R. Vassen, D. Stöver, J. Am. Ceram. Soc. 86, 1338-1344 (2003).

[7] B. Mandal, A. Tyagi, Barc Newsletter Issue 313, 6-13 (2010).

[8] J.K. Gill, O.P. Pandey, K. Singh, Int. J. Hydrog. Energy 37, 3857-3864 (2012).

[9] T. V. Barinova, I.P. Borovinskaya, V.I. Ratnikov, T.I. Ignat'eva, a. F. Belikova, N. V. Skachkova, N.Y. Khomenko, Int. J. Self-Propagating HighTemperature Synth. 20, 67-71 (2011).

[10] O. Merka, D.W. Bahnemann, M. Wark, Improved, ChemCatChem. 4, 18191827 (2012).

[11] M. Saif, M. Shebl, A.I. Nabeel, R. Shokry, H. Hafez, A. Mbarek, K. Damak, R. Maalej, M.S.A. Abdel-Mottaleb, B Chem. 220, 162-170 (2015).

[12] P. Dai, X. Zhang, M. Zhou, X. Li, J. Yang, P. Sun, C. Xu, Y. Liu, J. Am. 
Ceram. Soc. 95, 658-662 (2012).

[13] N. Pailhé, M. Gaudon, A. Demourgues, Mater. Res. Bull. 44, 1771-1777 (2009).

[14] F. Matteucci, G. Cruciani, M. Dondi, G. Baldi, A. Barzanti, Acta Mater. 55, 2229-2238 (2007).

[15] S. Ishida, F. Ren, N. Takeuchi, J. Am. Ceram. Soc. 76, 2644-2648 (1993).

[16] Z. Zhang, S.C. Middleburgh, M. de los Reyes, G.R. Lumpkin, B.J. Kennedy, P.E.R. Blanchard, E. Reynolds, L.-Y. Jang, J. Phys. Chem. C. 117, 2674026749 (2013).

[17] M.A. Subramanian, G. Aravamudan, G.V.S. Rao, Prog. Solid St. Chem. 15, 55-143 (1983).

[18] M.J.D. Rushton, R.W. Grimes, C.R. Stanek, S. Owens, J. Mater. Res. 19, 1603-1604 (2004).

[19] H.Y. Xiao, F.X. Zhang, F. Gao, M. Lang, R.C. Ewing, W.J. Weber, Phys. Chem. Chem. Phys. 12, 12472-12477 (2010).

[20] J. Lian, X.T. Zu, K.V.G. Kutty, J. Chen, L.M. Wang, R.C. Ewing, R.C. Ewing, Phys. Rev. B - Condens. Matter Mater. Phys. 66, 541081-541085 (2002).

[21] S.T. Norberg, S. Hull, S.G. Eriksson, I. Ahmed, F. Kinyanjui, J.J. Biendicho, Chem. Mater. 24, 4294-4300 (2012).

[22] H. Li, Q. Tao, N. Li, R. Tang, Y. Zhao, H. Zhu, P. Zhu, X. Wang, J. Alloys Compd. 660, 446-449 (2016).

[23] C.R. Stanek, Atomic Scale Disorder in Fluorite and Fluorite Related Oxides, PhD Thesys University of London (2003).

[24] W.R. Panero, L. Stixrude, R.C. Ewing, Phys. Rev. B. 70, 054110 (2004). 
[25] Z. Zhang, S.C. Middleburgh, M.D.L. Reyes, G.R. Lumpkin, B.J. Kennedy, P.E.R. Blanchard, E. Reynolds, J. Phys. Chem. 117, 26740-26749 (2013).

[26] K.E. Sickafus, R.W. Grimes, J.A. Valdez, A. Cleave, M. Tang, M. Ishimaru, S.M. Corish, C.R. Stanek, B.P. Uberuaga, Nat. Mater. 6, 217-223 (2007).

[27] P.O.L. Duwez, F.H. Brown, F. Odell, J Electrochem. Soc, 356-362 (2003).

[28] F. Fu-Kang, A.K.Kuznetsov, E.K. Keler, Chem.Sci. (Engl. Transl.) 20, 601610 (1963).

[29] H.G. Scott, J. Mater. Sci. 10, 1527-1535 (1975).

[30] C. Pascual, P. Duran, J. Am. Cer. Soc. 66, 23-27 (1982).

[31] V. S. Stubican, Adv. Ceram. 24A, 71 (1988).

[32] S.A. Degtyarev, G.F. Voronin, Calphad. 12, 73-82 (1988).

[33] Y. Du, Z. Jin, P. Huang, J. Am. Ceram. Soc. 74, 1569-1577 (1991).

[34] M. Chen, B. Hallstedt, L.J. Gauckler, Solid State Ionics. 170, 255-274 (2004).

[35] O. Fabrichnaya, S. Lakiza, C. Wang, M. Zinkevich, F. Aldinger, A, J. Alloys Compd. 453, 271-281 (2008).

[36] V.S. Stubican, R.C. Hink, S.P. Ray,J. Am. Ceram. Soc. 6, 17-21 (1978).

[37] H. Yokohawa, Key Eng. Mater. 153-154, 37-74 (1998).

[38] N.S. Jacobson, Z.K. Liu, L. Kaufman, F. Zhang,J. Am. Ceram. Soc. 87, 1559-1566 (2004).

[39] M. Yoshimura, J. Am. Ceram. Soc. Bull. 67,1950-1955 (1988).

[40] J.-P. Brog, C.-L. Chanez, A. Crochet, K.M. Fromm, RSC Adv. 3, 16905 (2013). 
[41] E.H. Kisi, C.J. Howard, Key Eng. Mater. 153-154, 1-36 (1998).

[42] R. Stevens, Magnesium Elektron 113, (1986).

[43] Binary alloy phase diagrams. Vol. 3, ASM International, (1990).

[44] G. Štefanić, S. Musić,Croat. Chem. Acta. 75, 727-767 ( 2002).

[45] M.J.Osendi, J.S.Moya, Bol. Soc. Esp. Ceram.Vidr. 21, 33-40 (1982).

[46] N. Shibata, J. Katamura, A. Kuwabara, Y. Ikuhara, T. Sakuma, Mater. Sci. Eng. A. 312, 90-98 (2001).

[47] S. Fabris, A.T. Paxton, M.W. Finnis, Acta Mater. 50, 5171-5178 (2002).

[48] A. Bogicevic, C. Wolverton, G.M. Crosbie, E.B. Stechel, Phys. Rev. B. 64, 1-14 (2001).

[49] J.R. Kelly, I. Denry, Dent. Mater. 24, 289-298 (2008).

[50] P. Li, I.-W. Chen, J.E. Penner-Hahn, J. Am. Ceram. Soc. 77, 1289-1295 (1994).

[51] A. Kuwabara, J. Katamura, Y. Ikuhara, T. Sakuma, J. Am. Ceram. Soc. 85, 2557-2561 (2002).

[52] G. Witz, V. Shklover, W. Steurer, S. Bachegowda, H.P. Bossmann, J. Am. Ceram. Soc. 90, 2935-2940 (2007).

[53] M. Gaudon, C. Laberty-Robert, F. Ansart, P. Stevens, A. Rousset, Solid State Sci. 5, 1377-1383 (2003).

[54] K. K.Srivastava, R. N. Patil, C. B. Choudhury, K. B. G. K. Gokhale and E. C. Subbarao, Trans J Br Ceram Soc. 73, 85-91 (1974).

[55] F.F. Lange, H. Shubert, N. Claussen, M. Ruhle, J. Mater. Sci. 21, 768-774 (1986).

[56] V. Srikanth, E.C. Subbarao, J. Mater. Sci. 29, 3363-3371 (1994). 
[57] R.Ruh, K.S. Mazdiyasni, P.G Valentine, H.O Bielstein Com, J. Am. Cer. Soc. 6, 190-192 (1984).

[58] H.G. Scott, J. Mater. Sci.12, 311-316 (1977).

[59] M. Jayaratna, M. Yoshimura, S.Somiya, Com. J. Am. Cer. Soc. 240-242, (1984).

[60] R.S. Roth, J. Res Natl. Bur. Std. 56, 17-25 (1956).

[61] D.K. Smith, J. Am. Ceram. Soc. 49, 48-49 (1966).

[62] X. Zou, G. Zhou, H. Yi, G. Zhang, S. Wang, J. Am. Ceram. Soc. 94, 10021004 (2011).

[63] M. Kumar, I.A. Raj, R. Pattabiraman, Mater. Chem. Phys. 108, 102-108 (2008).

[64] M. Kumar, M.A. Kulandainathan, I.A. Raj, R. Chandrasekaran, R. Pattabiraman, Mater. Chem. Phys. 92, 295-302 (2005).

[65] L. Gao, Y. An, H. Zhu, L. Wang, J. Chen, N. Wang, G. Ou, J. Mater. Sci. 46, 1337-1340 (2010).

[66] P. Escribano, J.B.C. Castello, E. Cordoncillo, Esmaltes y Pigmentos Ceramicos, Faenza Editrice Ibérica (2001).

[67] F. Melero, Materiales y procesos avanzados. Materiales de alta tecnología, Consejo Superior de Investigaciones Científicas (1993).

[68] J. Livage, C. Sanchez, J. Non. Cryst. Solids. 145, 11-19 (1992).

[69] C. Sanchez, J. Livage, M. Henry, F. Babonneau, J. Non. Cryst. Solids. 100, 65-76 (1988).

[70] U. Schubert, Chemistry and Fundamentals of the Sol-Gel Process in: SolGel Handbook (2015). 
[71] S. Chakrabarti, J. Sahu, A. Biswas, H.N. Acharya, J. Mater. Sci. Lett. 11, 763-766 (1992).

[72] M.S. Andrés, N. Sancho, An. La Real Soc. Española Química 106, 58-65 (2010).

[73] G. Monros, Encyclopedia of Color Science and Technology, 1-15 (2016).

[74] G. Monrós, El Color de la cerámica : nuevos mecanismos en pigmentos para los nuevos procesados de la industria cerámica, Publicacions de la Universitat Jaume I. Servei de Comunicació i Publicacions, Castelló de la Plana (2003).

[75] J.M.G. Vallejo, A.H. Gismero, Técnicas fisicoquímicas en medio ambiente, UNED (2011).

[76] D.C.M.A.M. Oxides, C.C. Subcommittee, DCMA Classification and Chemical Description of the Mixed Metal Oxide Inorganic Colored Pigments, Metal Oxides and Ceramic Colors Subcommittee, Dry Color Manufacturers' Association (1982).

[77] J. Godt, F. Scheidig, C. Grosse-Siestrup, V. Esche, P. Brandenburg, A. Reich, D.A. Groneberg, J. Occup. Med. Toxicol. 1, 1-6 (2006).

[78] ECHA (European Chemicals Agency), ANNEX XVII TO REACH. Conditions of restriction, 63-65 (2010).

[79] J. Wu, K. Li, X. Xu, J. Yu, X. Li, J. Tian, Mater. Chem. Phys. 203, 193-201 (2018).

[80] J. Wu, K. Li, X. Xu, Y. Zhang, X. Xu, X. Lao, J. Wuhan Univ. Technol. Mater. Sci. 30, 1247-1254 (2015).

[81] R.M. Cornell, U. Schwertmann, The Iron Oxides:Structure, Properties, Reactions, Occurrences and Uses Wiley-VCH (2003). 
[82] M. Llusar, J.A. Badenes, J. Calbo, M.A. Tcna, G. Monros, E Br. Ceram. Trans. 99, 14-22 (2000).

[83] K. Pyon, B. Lee, J. Cerm. Soc. Jap. 117, 258-263 (2009).

[84] G. Cappelletti, S. Ardizzone, P. Fermo, S. Gilardoni, J. Eur. Ceram. Soc. 25, 911-917 (2005).

[85] E. Carreto, C. Piña, H. Arriola, C. Barahona A., N. Nava, V. Castaño, J. Radioanal. Nucl. Chem. 250, 453-458 (2001).

[86] R. Eppler, J. Am. Cer. Soc 62, 47-49 (1979).

[87] B. Huang, Y. Xiao, C. Huang, J. Chen, X. Sun, Dye. Pigment. 147, 225-233 (2017).

[88] A.K. V Raj, P. Prabhakar Rao, S. Sameera, S. Divya, Dye. Pigment. 122, 116-125 (2015).

[89] C. Gargori García, S. Cerro Lloria, N. Fas Argamasilla, M. Llusar Vicent, G. Monrós Tomás, Bol. Nac. Soc. Esp. Ceram. Y Vidr. 56, 166-176 (2017).

[90] L.M. Schabbach, D.L. Marinoski, S. Güths, A.M. Bernardin, M.C. Fredel, Sol. Energy. 159, 113-124 (2018).

[91] A.K. Bendiganavale, V.C. Malshe, Recent Patents on Chemical Engineering, 67-79 (2008).

[92] M.Jansen, H.P. Letschert, Nature 404, 980-982 (2000).

[93] T. Thongkanluang, Int. J. Appl. Ceram. Technol 8, 1451-1458 (2011).

[94] T. Thongkanluang, P. Limsuwan, P. Rakkwamsuk, J. Cerm. Soc. Jap 118, 349-352 (2010).

[95] G. George, V.S. Vishnu, M.L.P. Reddy, Dye. Pigment. 88, 109-115 (2011).

[96] L.N. Sun, H.J. Zhang, Q.G. Meng, F.Y. Liu, L.S. Fu, C.Y. Peng, J.B. Yu, 
G.L. Zheng, S. Bin Wang, J. Phys. Chem. B. 109, 6174-6182 (2005).

[97] R. Iano, R. Lazu, P. Barvinschi, Adv. Powder Technol. 22, 396-400 (2011).

[98] G.A. Molander, Chem. Rev. 92, 29-68 (1992).

[99] M. Shibasaki, N. Yoshikawa, Chem. Rev. 102, 2187-2209 (2002).

[100] F. Hulliger, H.R. Ott,Phys. B Condens. Matter. 67, 291-298 (1987).

[101] M. Machida, S. Murakami, T. Kijima, S. Matsushima, M. Arai, J. Phys. Chem. B 105, 3289-3294 (2001).

[102] C.H. Evans, Trends Biochem. Sci. 8, 445-449 (1983).

[103] M. Martos, B. Julian-López, E. Cordoncillo, P. Escribano, J. Phys. Chem. B. 112, 2319-25 (2008).

[104] M Martos, B. Julian-Lopez, E. Cordoncillo, P. Escribano, J. Am. Ceram. Soc. 92, 2987-2992 (2009).

[105] V. De la Luz, M. Prades, H. Beltrán, E. Cordoncillo, J. Eur. Ceram. Soc. 33, 3359-3368 (2013).

[106] A. García, M. Llusar, J. Calbo, M.A. Tena, G. Monrós, Green Chem. 3, 238242 (2001).

[107] L. Yuan, A. Han, M. Ye, X. Chen, L. Yao, C. Ding, Dye. Pigment. 148, 137-146 (2018).

[108] A.K.V. Raj, P. Prabhakar Rao, S. Divya, T.R. Ajuthara, Powder Technol. 311, 52-58 (2017).

[109] M. Zhao, A. Han, M. Ye, T. Wu, Sol. Energy 97, 350-355 (2013).

[110] L. Stránská, P. Šulcová, M. Vlček,J. Therm. Anal. Calorim. 113, 127-135 (2013). 
[111] Q. Du, G. Zhou, J. Zhou, X. Jia, H. Zhou, J. Alloys Compd. 552, 152-156 (2013).

[112] M. Saif, J. Lumin. 135, 187-195 (2013).

[113] J. Papan, K. Vuković, S.P. Ahrenkiel, D.J. Jovanović, M.D. Dramićanin, J. Alloys Compd. 712, 437-444 (2017).

[114] A. Zhang, M. Lü, Z. Qiu, Y. Zhou, Q. Ma, Mater. Chem. Phys. 109, 105108 (2008).

[115] F.A. Kröger, H.J. Vink, Solid State Phys. Adv. Res. Appl. 3, 307-435 (1956).

[116] S.P.S. Badwal, Solid State Ionics 52, 23-32 (1992).

[117] N. Mahato, A. Banerjee, A. Gupta, S. Omar, K. Balani, Prog. Mater. Sci. 72, 141-337 (2015).

[118] J.M. Dixon, L.D. LaGrange, U. Merten, C.F. Miller, J.T. Porter, J. Electrochem. Soc. 110, 276 (1963).

[119] Y. Yamamura, Molecular dynamics analysis of ionic conduction mechanism in yttria-stabilized zirconia, Solid State Ionics. 126, 181-189 (1999).

[120] C. Haering, A. Roosen, H. Schichl, Solid State Ionics. 176, $253-259$ (2005).

[121] T. Arima, K. Fukuyo, K. Idemitsu, Y. Inagaki, J. Mol. Liq. 113, 67-73 (2004).

[122] M. Jovaní, H. Beltrán-Mir, E. Cordoncillo, A.R. West, Inorg. Chem. 56, 7081-7088 (2017).

[123] J. Schefold, A. Brisse, M. Zahid, J. Electrochem. Soc. 156, B897-B904 (2009).

[124] A.R. West, Solid state chemistry and its applications, 2nd ed. Wiley (2014). 
[125] M. Prades, N. Maso, H. Beltran, E. Cordoncillo, A.R. West, J. Mater. Chem 20, 5335-5344 (2010).

[126] N. Maso, H. Beltran, M. Prades, E. Cordoncillo, A.R. West,A.R. West, Phys. Chem. Chem. Phys.16, 19408-19416 (2014).

[127] Z. Suo, J. Mech. Phys. Solids. 41, 1155-1176 (1993).

[128] X.-B. Yin, Z.-H. Tan, X. Guo, Phys. Chem. Chem. Phys. 17, 134-137 (2015).

[129] D.B. Strukov, G.S. Snider, D.R. Stewart, R.S. Williams, Nature 453, 80 (2008).

[130] N. Masó, M. Prades, H. Beltrán, E. Cordoncillo, D.C. Sinclair, A.R. West, Appl. Phys. Lett. 97, 1-4 (2010).

[131] H. Beltrán, M. Prades, N. Masó, E. Cordoncillo, A.R. West, J. Am. Ceram. Soc. 93, 500-505 (2010).

[132] L. Gil Escrig, M. Prades, H. Beltran, E. Cordoncillo, N. Mas, A.R. West, J. Am. Ceram. Soc. 97, 2815-2824 (2014).

[133] N. Masó, A.R. West, Chem. Mater. 24, 2127-2132 (2012).

[134] N.Q. Minh, Ceramic Fuel Cells, J. Am. Ceram. Soc. 76, 563-588 (1993).

[135] R.M. Ormerod, Chem. Soc. Rev. 32, 17-28 (2003).

[136] B.C.H. Steele, J. Power Sources. 49, 1-14 (1994).

[137] N. Maso, A.R. West, Chem. Mater. 27, 1552-1558 (2015).

[138] W. Lai, S.M. Haile, J. Am. Ceram. Soc. 88, 2979-2997 (2005).

[139] J. Jamnik, J. Maier, J. Electrochem. Soc. 146, 4183-4188 (1999).

[140] Ž. Dohnalová, P. Šulcová, M. Trojan,J. Therm. Anal. Calorim. 101, 973978. (2010). 
[141] M. Llusar, L. Vitásková, P. Šulcová, M.A. Tena, J.A. Badenes, G. Monrós, J. Eur. Ceram. Soc. 30, 37-52. (2010).

[142] A. Fernández-Osorio, C.E. Rivera, A. Vázquez-Olmos, J. Chávez, Dye. Pigment. 119, 22-29 (2015).

[143] A. Lira-Campos, M. Mayorga-Rojas, Rev. Mex. Física. E57, 114-118. (2011).

[144] G. Wakefield, H.A. Keron, P.J. Dobson, J.L. Hutchison, J. Phys. Chem. Solids 60, 503-508 (1999).

[145] P.A.M. Berdowski, M.J.J. Lammers, G. Blasse, Chem. Phys. Lett. 113, 387390 (1985).

[146] J.C.Krupa, N.A. Kulagin, Physiscs of Laser Crystals, Springer Netherlands (2003).

[147] P. Dorenbos, E. van der Kolk, Opt. Mater. 30, 1052-1057 (2008).

[148] H.Hoefdraad, Charge-Transfer Spectra of lanthanide ions in oxides, $\mathrm{PhD}$ Thesys Willemstad (1947).

[149] K. Fuji and K. Sono, Repts. Govt. Res. Inst. Ceram 6, 18 (1952).

[150] J.A. Badenes, M. Llusar, J.B. Vicent, M.A. Tena, G. Monros, J. Mater. Sci. 37, 1413-1420 (2002)

[151] J.A. Badenes, M. Llusar, M.A. Tena, J. Calbo, G. Monrós,J. Eur. Ceram. Soc. 22, 1981-1990 (2002).

[152] J.K. Kar, R. Stevens, C.R. Bowen, Adv. Appl. Ceram. 104, 233-238 (2013).

[153] N. Montoya, G. Herrera, J. Alarcon,Ceram. Int. 37, 3609-3616 (2011).

[154] D. Guo, Q. Yang, P. Chen, Y. Chu, Y. Zhang, P. Rao, Dye. Pigment. 153, 74-83 (2018). 
[155] L. Sandhya Kumari, P. Prabhakar Rao, M. Lakshmipathy Reddy, J. Alloys Compd. 461, 509-515 (2008).

[156] R.C. Olegário, E.C. Ferreira De Souza, J.F. Marcelino Borges, J.B. Marimon Da Cunha, A.V. Chaves De Andrade, S.R. Masetto Antunes, A.C. Antunes, Dye. Pigment. 97, 113-117 (2013).

[157] H. Takashima, K. Ueda, M. Itoh, Appl. Phys. Lett. 89, 1-4 (2006).

[158] P.W. Metz, K. Hasse, D. Parisi, N. Hansen, C. Kränkel, M. Tonelli, G. Huber, Opt. Lett.39, 5158-5161 (2014).

[159] J. Oliva, E. De La Rosa, L.A. Diaz-Torres, A. Torres, P. Salas, O. Meza, J. Lumin. 154, 185-192 (2014).

[160] P. Dai, C. Ji, L. Shen, Q. Qian, G. Guo, X. Zhang, N. Bao, J. Rare Earths. 35, 341-346 (2017).

[161] A.A. Kaminskii, H.J. Eichler, B. Liu, P. Meindl, Phys. Stat.Sol. 138, K45K48 (1993).

[162] P. Boutinaud, R. Mahiou, E. Cavalli, M. Bettinelli, J. Lumin. 122-123, 430433 (2007).

[163] F. Ramos-Brito, H.S. Murrieta, J.A. Hernández, E. Camarillo, M. GarcíaHipólito, R. Martínez-Martínez, O. Álvarez-Fragoso, C. Falcony, J. Phys. D. Appl. Phys. 39, 2079-2083 (2006).

[164] Q. Du, G. Zhou, H. Zhou, Z. Yang, Opt. Mater. 35, 257-262 (2012).

[165] M. Jovani, A. Sanz, H. Beltran-Mir, E. Cordoncillo, Dye. Pigment. 133, 3340 (2016).

[166] J.H. Lee, Y.J. Kim, Ceram. Int. 34, 1113-1116 (2008).

[167] U. Kempe, S.M. Thomas, G. Geipel, R. Thomas, M. Plötze, R. Böttcher, G. Grambole, J. Hoentsch, M. Trinkler, Am. Mineral. 95, 335-347 (2010). 
[168] M. Dondi, F. Matteucci, I. Zama, G. Cruciani, Mater. Res. Bull. 42, 64-76 (2007).

[169] M. Dondi, F. Matteucci, G. Baldi, A. Barzanti, G. Cruciani, I. Zama, C.L. Bianchi, Dye. Pigment 76, 179-186 (2008).

[170] M. Descostes, F. Mercier, N. Thromat, C. Beaucaire, M. Gautier-soyer, Appl. Surf. Sci. 165, 288-302 (2000).

[171] T. Yamashita, P. Hayes, Appl. Surf. Sci. 254, 2441-2449 (2008).

[172] P.C.J. Graat, M.A.J. Somers, Appl. Surf. Sci. 100-101, 36-40 (1996).

[173] C.D. Wagner, L.H. Gale, R.H. Raymond, Anal. Chem. 51, 466-482 (1979).

[174] L.S. Kumari, P.P. Rao, P. Koshy, J. Am. Ceram. Soc. 93, 1402-1408 (2010).

[175] L.S. Kumari, T.H. Gayathri, S.F. Sameera, P.P. Rao, J. Am. Ceram. Soc. 94, 320-323 (2011).

[176] J. Zou, P. Zhang, C. Liu, Y. Peng, Dye. Pigment. 109, 113-119 (2014).

[177] Y. Li, S. Mei, Y. Byon, J. Wang, G. Zhang, Highly S ACS Sustainable Chem. Eng 2, 318-321 (2014).

[178] A. Han, M. Zhao, M. Ye, J. Liao, Z. Zhang, N. Li, Sol. Energy 91, 32-36 (2013).

[179] V. Sarasamma Vishnu, M. Lakshmipathi Reddy, Sol. Energy Mater. Sol. Cells. 95, 2685-2692 (2011).

[180] M. Kumar, M.A. Kulandainathan, I.A. Raj, R. Chandrasekaran, R. Pattabiraman, Mater. Chem. Phys. 92, 303-309 (2005).

[181] E. Gilardi, G. Gregori, Y. Wang, W. Sigle, P.A. van Aken, J. Maier, ACS Appl. Mater. Interfaces 9(32), 27257-27265 (2017). 
[182] A.B. Stambouli, E. Traversa, J. Renew. Sustain. Energy Rev., 6, 433-455 (2002).

[183] M.A. Hernandez, A.R. West, J. Mater. Chem. A Mater. Energy Sustain. 4, 1298-1305 (2016).

[184] J.A. Kilner, B.C.H. Steele, Academic. Press. In. (1981).

[185] R.L. Carlin, Introduction to ligand field theory (Ballhausen. Carl J.), J. Chem. Educ. 40, 390 (1963)

[186] W. Liu, J. Ji, H. Chen, C. Ye, PLoS One. 9, 1-9 (2014).

[187] A. Valea Pérez, J.M. Alonso Girón, McGraw-Hill, Madrid (1998).

[188] D.A. Skoog, J.J. Leary, C. Ariño Blasco , Análisis instrumental, ed, McGraw-Hill, Madrid (1994).

[189] D. Lin, Self-assembled nanostructures for bioanalysis Defence Research and Development Canada (2015).

[190] J.T.S. Irvine, D.C. Sinclair, A.R. West, Adv. Mater. 2, 132-138 (1990). 


\section{RENUNCIA EXPRESA DE LOS COAUTORES}

La ciencia es la progresiva aproximación del hombre al mundo real.

Max Planck 



\section{I UNIVERSITAT \\ JAUME I}

Escola de Doctorat $\cdot$ ED

Ana Sanz, como coautor/ coautora doy mi autorización a Marcos Jovaní Beltrán para la presentación de las siguientes publicaciones como parte de su tesis doctoral.

Relación de publicaciones:

-New red-shade environmental-friendly multifunctional pigment base don Tb and $\mathrm{Fe}$ doped $\mathrm{Y}_{2} \mathrm{Zr}_{2} \mathrm{O}_{7}$ for ceramic applications and cool roof coatings.

Asimismo, renuncio a poder utilizar estas publicaciones como parte de otra tesis doctoral.

Y para que conste firmo el presente documento,

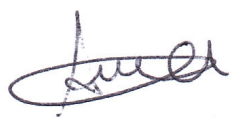

Castellón de la Plana, 25 de Julio de 2018

Todo ello, atendiendo al artículo 23 de la Normativa de los Estudios de Doctorado, regulados por el RD 99/2011, en la Universitat Jaume I (Aprobada por el Consejo de Gobierno núm. 19 de 26 de Enero de 2012, modificada por el Consejo de Gobierno núm. 29 de 27 de Noviembre de 2012 y con posterior modificación por el Consejo de Gobierno núm. 37 de 25 de Julio de 2013):

$$
\text { "(...) }
$$

"Aquellas tesis doctorales que opten por la incorporación de artículos (compendio de publicaciones) deben de ajustarse, en la medida de lo posible, a la siguiente estructura: -Introducción/objetivos - Un capítulo por artículo incorporado - Discusión general de los resultados - Conclusiones. -Aceptación de los coautores de que el doctorando presente el trabajo como tesis y renuncia expresa de estos a presentarlo como parte de otra tesis doctoral." 


\section{I UNIVERSITAT \\ JAUME I}

Escola de Doctorat $\cdot$ ED

Maria Fortuño Morte, como coautor/ coautora doy mi autorización a Marcos Jovaní Beltrán para la presentación de las siguientes publicaciones como parte de su tesis doctoral.

Relación de publicaciones:

\section{-Environmental-friendly red-orange ceramic pigment based on $\mathrm{Pr}$ and $\mathrm{Fe} \mathrm{co}-$ doped $\mathrm{Y}_{2} \mathrm{Z}_{2} \mathrm{O}_{7}$.}

Asimismo, renuncio a poder utilizar estas publicaciones como parte de otra tesis doctoral.

Y para que conste firmo el presente documento,

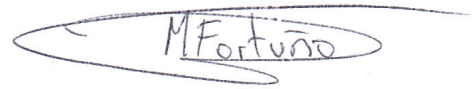

Castellón de la Plana, 25 de Julio de 2018

Todo ello, atendiendo al artículo 23 de la Normativa de los Estudios de Doctorado, regulados por el RD 99/2011, en la Universitat Jaume I (Aprobada por el Consejo de Gobierno núm. 19 de 26 de Enero de 2012, modificada por el Consejo de Gobierno núm. 29 de 27 de Noviembre de 2012 y con posterior modificación por el Consejo de Gobierno núm. 37 de 25 de Julio de 2013): "(...)

"Aquellas tesis doctorales que opten por la incorporación de artículos (compendio de publicaciones) deben de ajustarse, en la medida de lo posible, a la siguiente estructura: -Introducción/objetivos - Un capítulo por artículo incorporado - Discusión general de los resultados - Conclusiones. -Aceptación de los coautores de que el doctorando presente el trabajo como tesis y renuncia expresa de estos a presentarlo como parte de otra tesis doctoral." 


\section{I UNIVERSITAT JAUME I}

Escola de Doctorat $\cdot$ ED

Anthony R. West, como coautor/ coautora doy mi autorización a Marcos Jovaní Beltrán para la presentación de las siguientes publicaciones como parte de su tesis doctoral.

Relación de publicaciones:

\section{-Atmosphere- and Voltage-Dependent Electronic Condutivity of Oxide-Ion- Conducting $\mathrm{Zr}_{1-\mathrm{x}} \mathrm{Y}_{\mathrm{x}} \mathrm{O}_{2-\mathrm{x} / 2}$}

Asimismo, renuncio a poder utilizar estas publicaciones como parte de otra tesis doctoral.

Y para que conste firmo el presente documento,

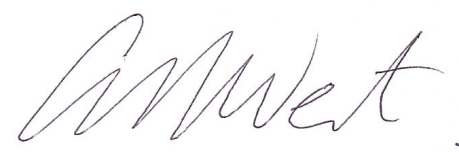

Sheffield, 24 de Enero de 2018

Todo ello, atendiendo al artículo 23 de la Normativa de los Estudios de Doctorado, regulados por el RD 99/2011, en la Universitat Jaume I (Aprobada por el Consejo de Gobierno núm. 19 de 26 de Enero de 2012, modificada por el Consejo de Gobierno núm. 29 de 27 de Noviembre de 2012 y con posterior modificación por el Consejo de Gobierno núm. 37 de 25 de Julio de 2013): “(...)

"Aquellas tesis doctorales que opten por la incorporación de artículos (compendio de publicaciones) deben de ajustarse, en la medida de lo posible, a la siguiente estructura: -Introducción/objetivos - Un capítulo por artículo incorporado - Discusión general de los resultados - Conclusiones. -Aceptación de los coautores de que el doctorando presente el trabajo como tesis y renuncia expresa de estos a presentarlo como parte de otra tesis 


\section{UNIVERSITAT \\ JAUME I}

Escola de Doctorat · ED

Héctor Beltrán Mir, como coautor/ coautora doy mi autorización a Marcos Jovaní Beltrán para la presentación de las siguientes publicaciones como parte de su tesis doctoral.

Relación de publicaciones:

-New red-shade environmental-friendly multifunctional pigment based on Tb and Fe doped $\mathrm{Y}_{2} \mathrm{Zr}_{2} \mathrm{O}_{7}$ for ceramic applications and cool roof coatings.

\section{-Atmosphere and Voltage-Dependent Electronic Conductivity of Oxide-Ion- Conducting $\mathrm{Zr}_{1-\times} \mathrm{Y}_{\mathrm{x}} \mathrm{O}_{2 \times \times 12}$}

\section{-Environmental-friendly red-orange ceramic pigment based on $\mathrm{Pr}$ and $\mathrm{Fe} \mathrm{co}=$ doped $\mathrm{Y}_{2} \mathrm{Zr}_{2} \mathrm{O}_{7}$}

Asimismo, renuncio a poder utilizar estas publicaciones como parte de otra tesis doctoral.

Y para que conste firmo el presente documento,

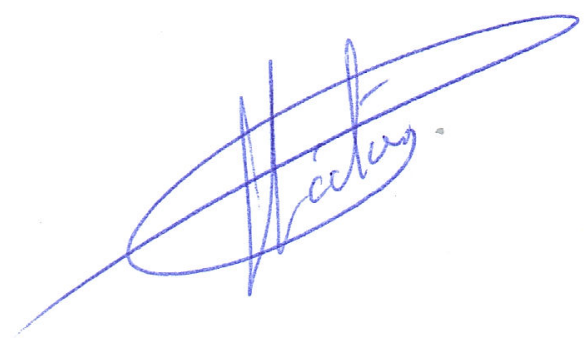

Castellón de la Plana, 25 de Septiembre de 2018

Todo ello, atendiendo al artículo 23 de la Normativa de los Estudios de Doctorado, regulados por el RD 99/2011, en la Universitat Jaume I (Aprobada por el Consejo de Gobierno núm. 19 de 26 de Enero de 2012, modificada por el Consejo de Gobierno núm. 29 de 27 de Noviembre de 2012 y con posterior modificación por el Consejo de Gobierno núm. 37 de 25 de Julio de 2013):

$$
\text { "(...) }
$$

"Aquellas tesis doctorales que opten por la incorporación de articulos (compendio de publicaciones) deben de ajustarse, en la medida de lo posible, a la siguiente estructura: -Introducción/objetivos - Un capitulo por articulo incorporado - Discusión general de los resultados - Conclusiones. -Aceptación de los coautores de que el doctorando presente el trabajo como tesis y renuncia expresa de estos a presentarlo como parte de otra tesis doctoral." 


\section{UNIVERSTAT \\ JAUME I}

Escola de Doctorat $\cdot E D$

Eloisa Cordoncillo Cordoncillo, como coautor/ coautora doy mi autorización a Marcos Jovaní Beltrán para la presentación de las siguientes publicaciones como parte de su tesis doctoral.

Relación de publicaciones:

-New red-shade environmental-friendly multifunctional pigment based on Tb and Fe doped $\mathrm{Y}_{2} \mathrm{Zr}_{2} \mathrm{O}_{7}$ for ceramic applications and cool roof coatings.

\section{-Atmosphere and Voltage-Dependent Electronic Conductivity of Oxide-lon- Conducting $\mathrm{Zr}_{1-\mathrm{x}} \mathrm{Y}_{\mathrm{x}} \mathrm{O}_{2 \times \times / 2}$}

-Environmental-friendly red-orange ceramic pigment based on $\mathrm{Pr}$ and $\mathrm{Fe} \mathrm{Co}=$ doped $\mathrm{Y}_{2} \mathrm{Zr}_{2} \mathrm{O}_{7}$.

Asimismo, renuncio a poder utilizar estas publicaciones como parte de otra tesis doctoral.

Y para que conste firmo el presente documento,

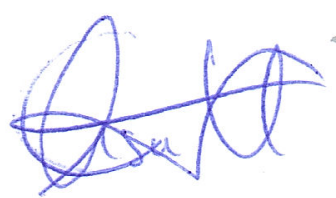

Castellón de la Plana, 25 de Septiembre de 2018

Todo ello, atendiendo al artículo 23 de la Normativa de los Estudios de Doctorado, regulados por el RD 99/2011, en la Universitat Jaume I (Aprobada por el Consejo de Gobierno núm. 19 de 26 de Enero de 2012, modificada por el Consejo de Gobierno núm. 29 de 27 de Noviembre de 2012 y con posterior modificación por el Consejo de Gobierno núm. 37 de 25 de Julio de 2013): "(...)

"Aquellas tesis doctorales que opten por la incorporación de artículos (compendio de publicaciones) deben de ajustarse, en la medida de lo posible, a la siguiente estructura: -Introducción/objetivos - Un capitulo por articulo incorporado - Discusión general de los resultados - Conclusiones. -Aceptación de los coautores de que el doctorando presente el trabajo como tesis y renuncia expresa de estos a presentarlo como parte de otra tesis doctoral." 



\section{PUBLICACIONES PREVIAS}





\title{
Pigments based on $\mathrm{Cr}$ and $\mathrm{Sb}$ doped $\mathrm{TiO}_{2}$ prepared by microemulsion- mediated solvothermal synthesis for inkjet printing on ceramics
}

\author{
Marc Jovaní a, María Domingo a , Thales R. Machado ${ }^{\mathrm{a}, \mathrm{b}}$, Elson Longo ${ }^{\mathrm{c}}$, \\ Héctor Beltrán-Mir ${ }^{a}{ }^{*}$, Eloisa Cordoncillo ${ }^{a}$ \\ a Departamento de Química Inorgánica y Orgánica, Universitat Jaume I de Castellón, Avda. Sos Baynat s/n, 12071 Castellón de la Plana, Spain \\ ${ }^{\mathrm{b}}$ LIEC-Laboratório Interdisciplinar de Eletroquímica e Cerâmica, Departamento de Química, UFSCar-Universidade Federal de São Carlos, Rod. Washington \\ Luis km 235, P.O. Box 676, 13565-905 São Carlos, São Paulo, Brazil \\ ${ }^{c}$ LIEC-Laboratório Interdisciplinar de Eletroquímica e Cerâmica, Instituto de Química, UNESP-Universidade Estadual Paulista Júlio de Mesquita Filho, P.O. \\ Box 355, 14801-907 Araraquara, São Paulo, Brazil
}

\section{A R T I C L E I N F O}

\section{Article history:}

Received 31 July 2014

Received in revised form

22 December 2014

Accepted 15 January 2015

Available online 23 January 2015

\section{Keywords}

Pigment

Titanium dioxide

Orange ceramic pigment

Inkjet

Ceramics

Solvothermal synthesis

\begin{abstract}
A B S T R A C T
A ceramic pigment with nominal composition $\mathrm{Ti}_{0.97} \mathrm{Cr}_{0.015} \mathrm{Sb}_{0.015} \mathrm{O}_{2}$ was prepared by microemulsionmediated solvothermal method at $180{ }^{\circ} \mathrm{C}$. Anatase or rutile single phase was obtained depending on the synthesis conditions. Scanning electron microscope analysis showed the formation of spheres with particle size around $600 \mathrm{~nm}$. The anatase to rutile transformation temperature was determined by XRD and Raman spectroscopy. The evolution of the colour was studied, and it was related with the polymorphic transition. Yellow pigments were obtained at low temperature and huge orange colour was observed at high temperature. Powders prepared at $180{ }^{\circ} \mathrm{C}$ were tested with an industrial frit. Similar chromatic coordinates of an industrial orange ceramic pigment obtained at high temperatures were observed. $\zeta$-potential values of the particles were $\sim-57 \mathrm{mV}$. The size, shape, colour and electrostatic stability of these particles make them potential candidates to be applied in glazes or inkjet printers as orange ceramic pigments.
\end{abstract}

() 2015 Elsevier Ltd. All rights reserved.

\section{Introduction}

Titanium dioxide, $\mathrm{TiO}_{2}$, has a wide range of applications. Since its commercial production in the early twentieth century, it has been widely used as a pigment [1], sunscreens [2,3], paints [4], toothpaste [5], etc. Titanium dioxide occurs mainly in three crystalline forms: rutile, anatase and brookite. Rutile is the stable phase, and anatase and brookite are metastable. Both anatase, space group I4/amd, and rutile, space group $\mathrm{P}_{2} / \mathrm{mnm}$, are tetragonal. Structures consist of $\mathrm{TiO}_{6}$ octahedra, sharing four edges in anatase and two edges in rutile $[6,7]$.

Nowadays, the field of nanotechnology has generated a great deal of interest because materials have numerous new properties in nanosize-scaled. These size-dependent properties include new phase transition behaviour, different thermal and mechanical properties, interesting surface activity and reactivity, and unusual optical, electrical and magnetic characteristics [8-10]. In this way,

\footnotetext{
* Corresponding author. Tel.: +34 964 728245; fax: +34 964728214

E-mail address: mir@uji.es (H. Beltrán-Mir).
}

and more closely in the field of pigments, $\mathrm{TiO}_{2}$ have a massive potential market. Study of the particle size of $\mathrm{TiO}_{2}$ is essential because it affects phase and thermal stability as well as surface and bulk properties [11-13]. The knowledge of the particle size is crucial to predict the behaviour of the nanoparticles in industrial processes, like in the case of ceramic decoration which the use of high temperatures are required [14].

Ceramic submicronic pigments have been developed for inkjet decoration of ceramic tiles using quadrichromic technology, being a new field of application. These pigments with particle size less than $1 \mu \mathrm{m}$ are able to overcome some actual problems of the inkjet industrial processes [15]. The use of pigmenting particles at the nanoscale is necessary for inkjet applications [16,17]. At present in the industry, these particles are basically obtaining by different milling steps, producing submicronic particles with no round shape $[18,19]$. The use of submicronic pigments in inkjet technology can solved problems like nozzle clogging, dispersion or instability caused by micronized pigments, and moreover, remove the milling stages [14].

Wet chemistry methods are one of the best options to prepare $\mathrm{TiO}_{2}$ nanoparticles. The literature reports approaches for the 
synthesis of nanoparticles of titania, including thermal hydrolysis $[20,21]$, sol-gel [22,23], hydro/solvothermal method [24,25] and microemulsion processes [26,27]. Among them, solvothermal and microemulsion methods are extensively used for the preparation of nanomaterials.

Solvothermal method has many advantages such as: (a) the final product can be obtained directly at relatively lower reaction temperature, (b) crystalline products with different composition, structure, and morphology could be prepared modifying the synthesis conditions like temperature, $\mathrm{pH}$, times or reactant concentration, and (c) it produces high purity particles compare with traditional solid-solid routes.

Therefore, the solvothermal synthesis is a good method for the preparation of oxide ceramic fine powders [24]. However, large sizes of $\mathrm{TiO}_{2}$ nanocrystals and poor dispersion stability usually appear in the materials prepared by solvothermal methods.

Microemulsions is a powerful method for obtaining ultrafine and nanometric particles with controlled size and shape [28]. $\mathrm{TiO}_{2}$ nanomaterials prepared by micelle method have often amorphous structure, and calcination is usually necessary in order to induce high crystallinity [10].

Based on the advantages of each method, a combination of microemulsion with solvothermal method has been explored to prepare nanomaterials such as $\mathrm{SrCO}_{3}$ nanostructures [29] or $\mathrm{Ca}_{10}\left(\mathrm{PO}_{4}\right)_{6}(\mathrm{OH})_{2}$ [30]. Microemulsions with nanosized aqueous cores have been used as the reaction media for preparation of $\mathrm{TiO}_{2}$ nanomaterials [31,32]. Shen et al. [33] have successfully synthesized rutile and anatase with microemulsion-mediated solvothermal method. They studied the preparation of rutile or anatase modifying the synthesis conditions such as the amount of urea in aqueous phase of the microemulsion. The effect of $\mathrm{pH}$ on $\mathrm{TiO}_{2}$ phase structure have been also studied [12,34]. Regarding these studies, the low pH favours the formation of rutile phase while more alkaline media favours anatase phase formation. Other studies were done in order to control the growth of the particles. Somiya et al. [35] prepared nanomaterials by hydrothermal microemulsion process in order to prevent grain growth of the nanoparticles. They conclude that the aqueous micelles in microemulsions act as microreactors to confine the growth of $\mathrm{TiO}_{2}$ powders.

As it mention before, $\mathrm{TiO}_{2}$ has been widely used as a pigment. In the industry, where the solid solution used is $\mathrm{Ti}_{0.97} \mathrm{Cr}_{0.015} \mathrm{Sb}_{0.015} \mathrm{O}_{2}$, the pigment is manufactured starting from anatase with chromium (III) oxide as chromophore element and antimony (III) oxide as counterions in presence of several mineralizers. In this case, the colour is acquired by calcinations at high temperatures around $1200^{\circ} \mathrm{C}$, where the development of the colour occurs during the anatase-rutile transformation [36]. Anatase to rutile transformation is reconstructive, therefore, transformation involves breaking and reforming bonds [7]. This reconstructive transformation involves a contraction of the c-axis and involving a volume contraction around 8\% [37].

In the literature, synthesis of $\mathrm{Cr}-\mathrm{Sb}-\mathrm{TiO}_{2}$ pigment was carried out by different preparation routes. $\mathrm{C}$. Gargoni et al.[38] prepared samples of composition $\mathrm{Ti}_{0.97} \mathrm{Cr}_{0.015} \mathrm{Sb}_{0.015} \mathrm{O}_{2}$ with rutile phase by several routes: ceramic method, heterogeneous ammonia coprecipitation method, homogeneous urea coprecipitation method, Pechini polyester method, and aqueous-organic coprecipitation method in a water-ditehylenglycol. Authors concluded that final colour in a conventional glaze from powders obtained by nonconventional methods was worse than colour obtained from powders prepared by the ceramic method. Other authors [14,39] used the polyol non-conventional method to synthesize nanoparticles of this pigment. Stable ceramic inks were prepared with a nanometric size by this method.

In this work, $\mathrm{Cr}$,Sb-doped $\mathrm{TiO}_{2}$ ceramic pigments were prepared by microemulsion-mediated solvothermal method. As far as we know, synthesis of this pigment has not been yet reported using this method. This route allows an accurate control of the phase and the particle size of the pigment, and therefore, it is possible to determine the influence of these properties to the final colour at high temperature in ceramic decoration. In spite of this method have been used to prepare $\mathrm{TiO}_{2}$, the incorporation of the $\mathrm{Cr}$ and $\mathrm{Sb}$ to this structure have not been reported using this method. In the literature [33] and, as well as in this study, the phase (anatase or rutile) was controlled by the $\mathrm{pH}$ of the aqueous phase. However, time of the solvothermal treatment has been studied in this work due to the presence of the chromophores ions. These ions must be incorporated in the structure to form a solid solution, and therefore, they can also affect to the final phase present and the kinetic conditions of the reaction. Due to it, the time of the reaction is an important parameter to consider here. Studies of the rutile-anatase transformation were also conducted in this work.

Microemulsion-mediated solvothermal method has been able to synthesise pigments with optimal colouration, controlling the phase, shape and size of the particles to be applied in inkjet printing of ceramics. For this application, the inorganic pigment was obtained at high temperature by the traditional solid-solid reaction. However, in this method, calcinations were unnecessary because the solvothermal treatment promoting the crystallization of the phase at low temperatures. Therefore, these $\mathrm{Cr}, \mathrm{Sb}$-doped $\mathrm{TiO}_{2}$ submicroparticles obtained at low temperatures $\left(180{ }^{\circ} \mathrm{C}\right)$ would have potential applications in the field of ceramic inks.

\section{Experimental}

Samples of $\mathrm{Ti}_{0.97} \mathrm{Cr}_{0.015} \mathrm{Sb}_{0.015} \mathrm{O}_{2}$ solid solution were prepared by a microemulsion-mediated solvothermal route. The synthesis procedure was as follows: first, $10 \mathrm{~mL}$ of Triton X-100 (surfactant), $3 \mathrm{~mL}$ of $\mathrm{n}$-hexanol (cosurfactant $98 \%$ ) and $16 \mathrm{~mL}$ of cyclohexane (Sigma-Aldrich, $\geq 99.5 \%$ ) were mixed under magnetic stirring, making up the oil phase. Second, $2 \mathrm{~mL}$ of $\mathrm{TiCl}_{4}$ (Fluka, $\geq 99 \%$ ) solution, previously prepared in an acid medium of $\mathrm{HCl} 4 \mathrm{M}, 2 \mathrm{~mL}$ of $\mathrm{H}_{2} \mathrm{O}$ and the specifics amounts of $\mathrm{CrCl}_{3} \cdot 6 \mathrm{H}_{2} \mathrm{O}$ (Probus, 93\%) and $\mathrm{SbCl}_{3}$ (Sigma-Aldrich, $\geq 99 \%$ ), together with the necessary amount of urea (Fluka, $\geq 99.5 \%$ ) to obtain the desired phase, were mixed under magnetic stirring (aqueous phase). The amount of urea was fixed to $4.5 \mathrm{~g}$ and $1.5 \mathrm{~g}$ per gram of pigment to obtain anatase (A) or rutile $(R)$ single phase, respectively [33].

Then, the aqueous phase was added dropwise to the oil phase under stirring mediated a peristaltic pump at room temperature, forming a clear microemulsion. The microemulsion was mixed under magnetic stirring for $48 \mathrm{~h}$ and, then, placed in a Teflon-lined stainless steel autoclave and heated at $180{ }^{\circ} \mathrm{C}$ in an oven for variable times. The precipitate was collected by centrifugation and washed repeatedly with ethanol. After this process, samples were dried in air at room temperature. A scheme of the general preparation of the samples is shown in Fig. 1, and the different treatment conditions used in each case (time and the amount of urea) are shown in Table 1.

It is well known that the anatase-rutile phase transition involves a volume contraction, and it depends on variables such as size, morphology, etc. [7]. Therefore, in order to study the anatase-rutile phase transition in these samples, the powder with anatase phase obtained at $180^{\circ} \mathrm{C}$ was annealed at different temperatures between 750 and $1080^{\circ} \mathrm{C}$ for $2 \mathrm{~h}$ and cooled slowly inside the furnace.

Powders of samples at $180{ }^{\circ} \mathrm{C}$, where single phase and optimal colour were obtained, were mixed with one industrial frit (4\% in weight of the pigment) using water as a dispersing medium. Then, the dispersion was applied to white twice-fire bodies, to verify composition stability as a ceramic colourant. A commercial transparent frit was chosen. The frit composition used is given in Table 2. 


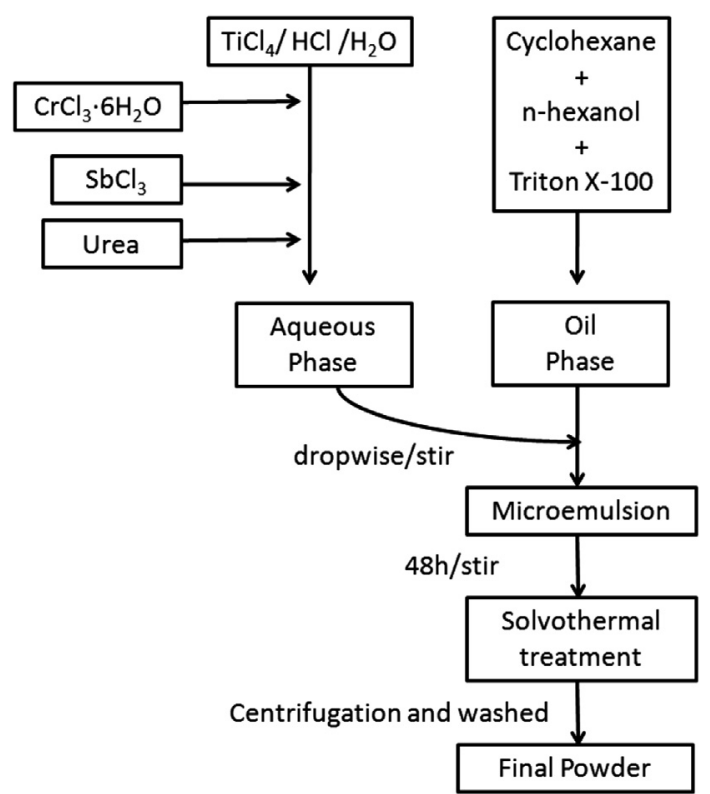

Fig. 1. Scheme of the $\mathrm{TiO}_{2}$ sample preparation.

After drying, the pieces were fired in an electric kiln. The heat treatment applied, corresponds to a standard firing cycle used in a ceramic tile industry where the highest temperature of the cycle was $1080{ }^{\circ} \mathrm{C}$ for $5 \mathrm{~min}$. This cycle involve five steps: ramping to $800^{\circ} \mathrm{C}$ in $18 \mathrm{~min}$, heating from $800^{\circ} \mathrm{C}$ to glaze firing temperature in $17 \mathrm{~min}, 5 \mathrm{~min}$ hold at $1080{ }^{\circ} \mathrm{C}$, cooling to $600{ }^{\circ} \mathrm{C}$ in $20 \mathrm{~min}$, and finally cooling to room temperature in $15 \mathrm{~min}$.

\subsection{Characterization}

Phase analysis of the samples was performed by powder XRD using a Bruker D4 Endeavour diffractometer with $\mathrm{CuK}_{\alpha}$ radiation. Data were collected by step-scanning from $2 \theta=20$ to $70^{\circ}$ with a step size of $0.05^{\circ}$ and $1.5 \mathrm{~s}$ of counting time at each step.

Scanning electron micrographs of the samples were taken on a field emission scanning electron microscope (SEM) JEOL 7001F, equipped with a spectrometer of energy dispersion of X-ray (EDX) from Oxford instruments, using acceleration voltage $=15 \mathrm{kV}$. Samples for microstructures and microanalysis determinations were deposited in an aluminium holder and sputtered by platinum. Dynamic Light Scattering (DLS, ZetaSizer-NanoSeries Malvern Instruments, Malvern, UK) was also used to measure the $\zeta$-potential of the as-prepared powder samples.

Raman spectra were recorder on a RFS/100/S Bruker Fourier transform (FT-Raman) spectrometer, with a Nd:YAG laser excitation light at $1064 \mathrm{~nm}$ in a spectral resolution of $4 \mathrm{~cm}^{-1}$, in order to confirm the polymorphic phase (anatase or rutile).

UV-Visible diffuse reflectance spectroscopy and colourimetric study of the glazed fired samples were performed on a CARY 500 SCAN VARIAN spectrophotometer in the $200-800 \mathrm{~nm}$ range. $\mathrm{BaSO}_{4}$

Table 1

Different treatment conditions to obtain rutile (R) or anatase $(A)$ phase.

\begin{tabular}{lll}
\hline Ref. & Urea(g)/Pigment $(\mathrm{g})$ & Time $(\mathrm{h})$ \\
\hline R1 & 1.5 & 17 \\
R2 & 1.5 & 24 \\
R3 & 1.5 & 48 \\
A1 & 4.5 & 17 \\
A2 & 4.5 & 24 \\
A3 & 4.5 & 48 \\
\hline
\end{tabular}

Table 2

Frit composition.

\begin{tabular}{|c|c|c|c|c|c|c|}
\hline \multicolumn{7}{|c|}{ Composition (wt\%) } \\
\hline $\mathrm{SiO}_{2}$ & $\mathrm{Al}_{2} \mathrm{O}_{3}$ & $\mathrm{RO}^{\mathrm{b}}$ & $\mathrm{R}_{2} \mathrm{O}^{\mathrm{b}}$ & $\mathrm{ZnO}$ & $\mathrm{ZrO}_{2}$ & Temperature $/{ }^{\circ} \mathrm{C}$ \\
\hline 67 & 13 & 9.4 & 10 & 0.4 & 0.2 & 1080 \\
\hline
\end{tabular}

b $\mathrm{R}=$ alkaline or alkaline earth metals.

was used as a reference. Reflectance $\left(R_{\infty}\right)$ was converted to absorbance $(K / S)$ by the Kubelka-Munk equation: $K / S=2\left(1-R_{\infty}\right) \times 2 R_{\infty}^{-1}$ [40]. The positions of the main absorbance peaks in the optical spectra were determined through a deconvolution procedure that allowed obtaining more accurately values for the electronic transitions. The CIELab colour parameters $L^{*}, a^{*}$, and $b^{*}$ of the glazed fired compositions and the anatase powders at different temperatures were determined by coupling an analytical software for colour measurements to the Varian spectrophotometer, using a standard illuminant D65, to differentiate the pigment in terms of colour. $L^{*}$ is the lightness axis [black $(0)$ to white $(100)$ ], $a^{*}$ is the green $(<0)$ to red $(>0)$ axis, and $b^{*}$ is the blue $(<0)$ to yellow $(>0)$ axis.

\section{Results and discussion}

\subsection{Optimization of the crystalline phase and microstructural characterization}

Microemulsions were heated at $180{ }^{\circ} \mathrm{C}$ for variable times to optimize the preparation of a rutile or anatase single phase. Several
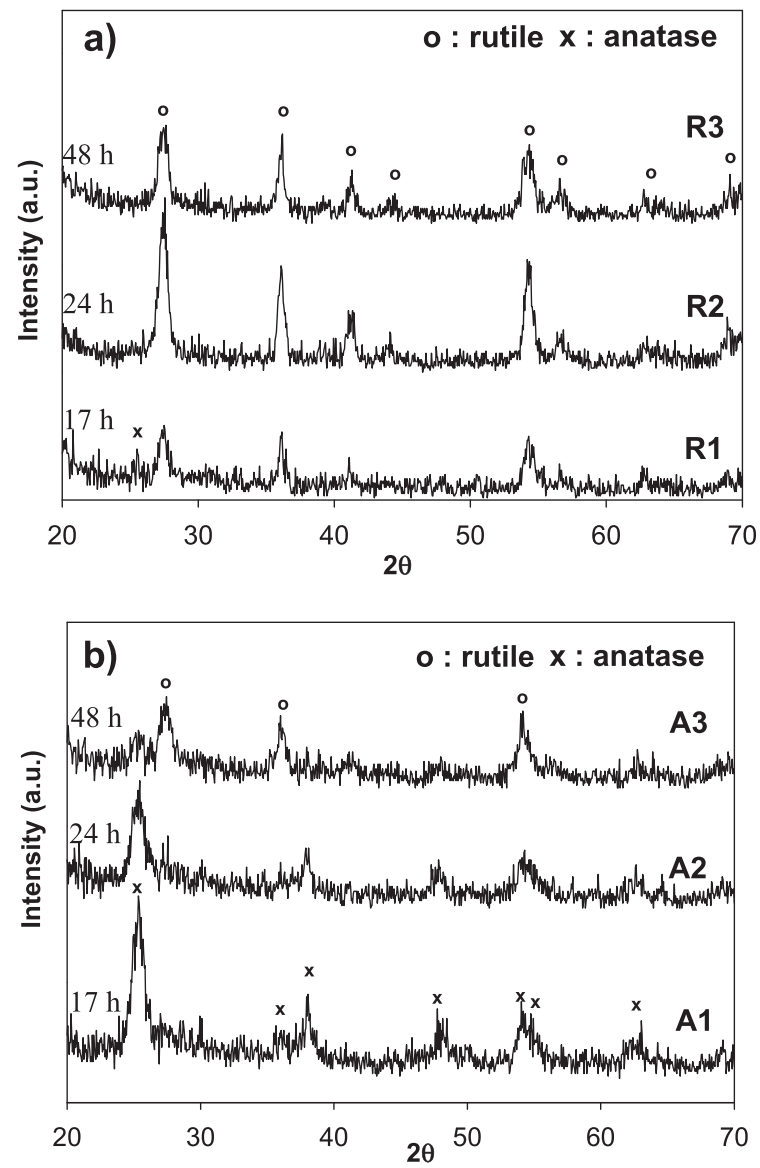

Fig. 2. XRD patterns of $\mathrm{TiO}_{2}$ synthesised with different amounts of urea and different times of reaction at $180{ }^{\circ} \mathrm{C}$ in order to prepare rutile (a) or anatase (b) phases. 

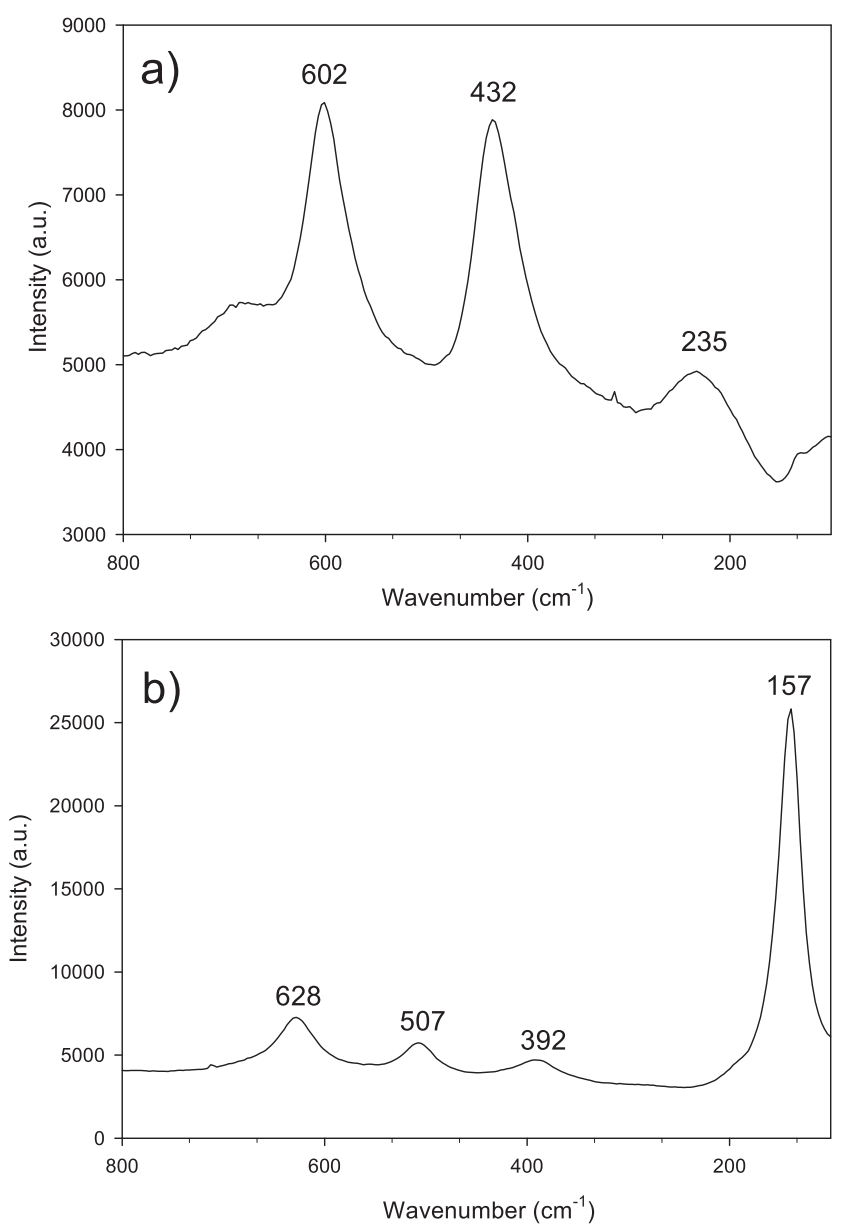

Fig. 3. Raman spectra for $\mathrm{TiO}_{2}$ samples prepared with (a) $1.5 \mathrm{~g}$ of urea and after $24 \mathrm{~h}$ of reaction, $\mathrm{R} 2$, and (b) $4.5 \mathrm{~g}$ of urea and after $17 \mathrm{~h}$ of reaction, A1. Rutile or anatase phases were observed in (a) and (b), respectively.
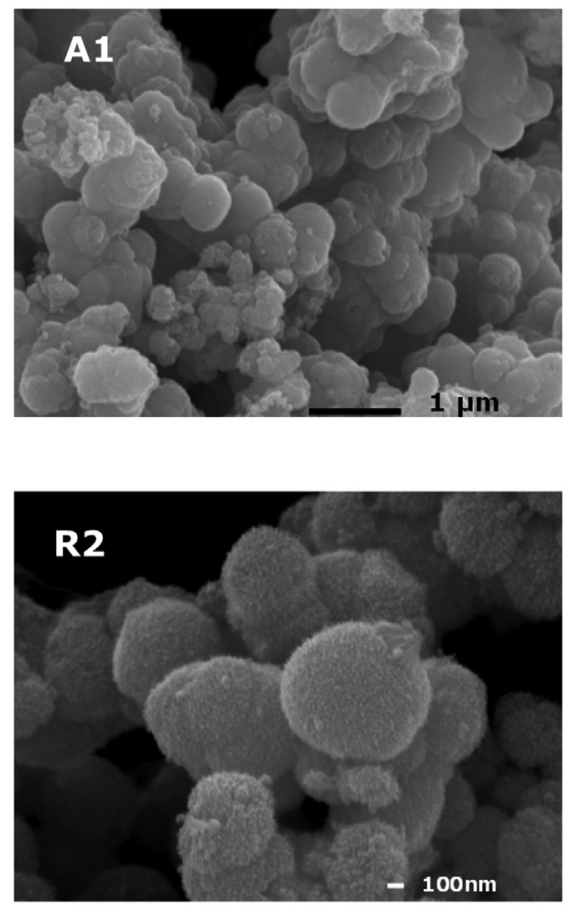
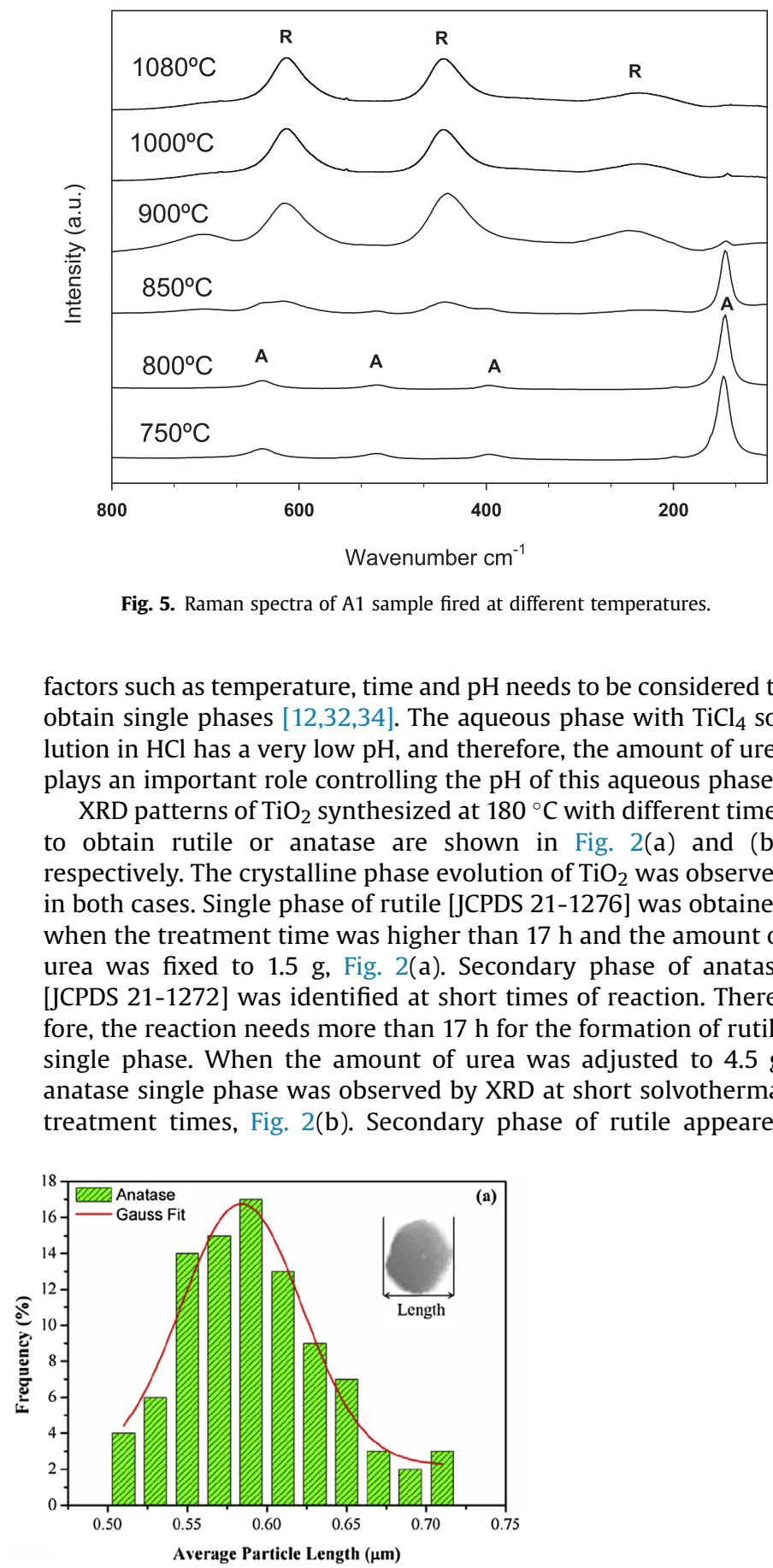

Fig. 5. Raman spectra of A1 sample fired at different temperatures.

factors such as temperature, time and $\mathrm{pH}$ needs to be considered to obtain single phases $[12,32,34]$. The aqueous phase with $\mathrm{TiCl}_{4}$ solution in $\mathrm{HCl}$ has a very low $\mathrm{pH}$, and therefore, the amount of urea plays an important role controlling the $\mathrm{pH}$ of this aqueous phase.

XRD patterns of $\mathrm{TiO}_{2}$ synthesized at $180{ }^{\circ} \mathrm{C}$ with different times to obtain rutile or anatase are shown in Fig. 2(a) and (b), respectively. The crystalline phase evolution of $\mathrm{TiO}_{2}$ was observed in both cases. Single phase of rutile [JCPDS 21-1276] was obtained when the treatment time was higher than $17 \mathrm{~h}$ and the amount of urea was fixed to $1.5 \mathrm{~g}$, Fig. 2(a). Secondary phase of anatase [JCPDS 21-1272] was identified at short times of reaction. Therefore, the reaction needs more than $17 \mathrm{~h}$ for the formation of rutile single phase. When the amount of urea was adjusted to $4.5 \mathrm{~g}$, anatase single phase was observed by XRD at short solvothermal treatment times, Fig. 2(b). Secondary phase of rutile appeared

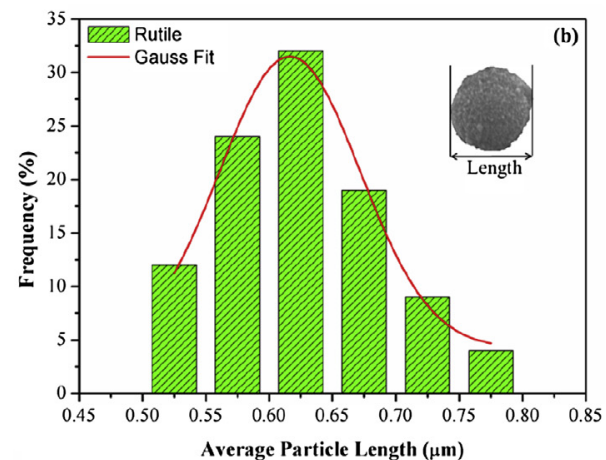

Fig. 4. SEM images of $A 1$ (a) and R2 (b) powder samples with the average particle size in each case. 
Table 3

Chromatic coordinates of A1 powder samples at different temperatures.

\begin{tabular}{llrl}
\hline & $\mathrm{L}^{*}$ & $\mathrm{a}^{*}$ & $\mathrm{~b}^{*}$ \\
\hline $180{ }^{\circ} \mathrm{C}(\mathrm{A} 1)$ & 93 & -5 & 21 \\
$750{ }^{\circ} \mathrm{C}$ & 78 & 0 & 23 \\
$800{ }^{\circ} \mathrm{C}$ & 76 & 1 & 23 \\
$850{ }^{\circ} \mathrm{C}$ & 77 & 3 & 28 \\
$900{ }^{\circ} \mathrm{C}$ & 77 & 5 & 31 \\
$1000{ }^{\circ} \mathrm{C}$ & 72 & 8 & 31 \\
$1080{ }^{\circ} \mathrm{C}$ & 70 & 14 & 36 \\
\hline
\end{tabular}

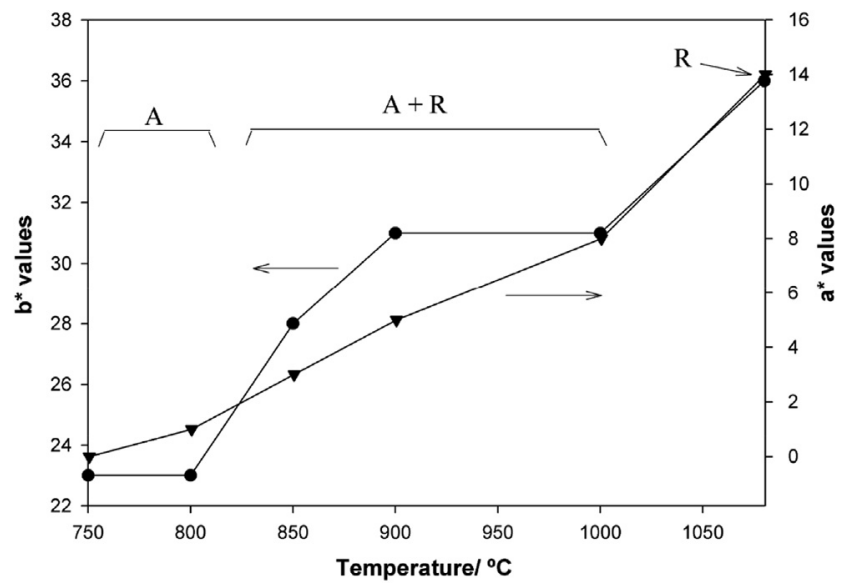

Fig. 6. CIELab chromatic coordinates of the A1 powder fired at different temperatures.

when the reaction time was increasing. R3 and R2 samples consist of single phase of rutile, while A1 sample is $100 \%$ anatase. $\mathrm{R} 2$ and A1 samples were chosen to continue the study due to both samples presents single phase by XRD and the shortest reaction times.

In order to confirm results obtained by $\mathrm{XRD}, \mathrm{TiO}_{2}$ powders were further characterized by Raman spectroscopy. Fig. 3 shows the Raman spectra of R2 and A1, showing the characteristic bands of the rutile and anatase single phase, respectively [41]. Peaks located at 235,432 and $602 \mathrm{~cm}^{-1}$ can be assigned to the rutile phase, Fig. 3(a). No peaks that could be assigned to anatase $\mathrm{TiO}_{2}$ or brookite were detected. In contrast, peaks located at 157, 392, 507 and $628 \mathrm{~cm}^{-1}$, Fig. 3(b), can be assigned to the anatase phase, and no other peaks were observed. Peaks that could be assigned to the doping oxides, such as $\mathrm{Cr}_{2} \mathrm{O}_{3}$, located at $550 \mathrm{~cm}^{-1}$ [42], were not found. These results were consistent with the XRD results shown in Fig. 2.

Products prepared by ceramic method at high temperature must be milled to adjust the grain size of the pigments in function of their applications. It is important to know the microstructure and grain size of the samples to select one or other application. For example, grain sizes of below one micron are required for inkjet applications [15]. Therefore, the average grain size of the R2 and A1 samples were analysed by SEM. Micrographs and the grain size distribution of both powder samples are shown in Fig. 4. In both cases, there was no evidence of secondary phases by EDX, and therefore, single-phase of $\mathrm{Cr}, \mathrm{Sb}-\mathrm{TiO}_{2}$ solid solutions were obtained. Spherical particles were observed and the grain size distribution was around $600 \mathrm{~nm}$ in both samples. Shape of the particles is also important for inkjet processes. Particles with rounded shape, as obtained in this work, are more suitable for inkjet fluid-dynamics instead of angular shapes, which are usually obtained from high energy ball milling. Production of inkjet inks involves a problem of the pigment sedimentation in the dispersant [15]. In this way, measurements of $\zeta$ potential were performed in water with $0.1 \%$ of sodium hexametaphosphate (65-70\%, Aldrich) [43], in order to determine the electrostatic stabilization of the R2 and A1 samples. Values of the $\zeta$-potential were $-57 \mathrm{mV}$ and $-56.5 \mathrm{mV}$, respectively. These negative values showed that the pigment can be dispersed, avoiding the sedimentation. $\zeta$-potential values around $20 \mathrm{mV}$ are obtained for the industrial pigment using glycol as dispersant [14]. Note that high $\zeta$-potential values (positive or negative) are better to avoid sedimentation and therefore, to be applied in inkjet technology. But, other important properties such as surface tension, density or viscosity of the ink should be consider before the application [11].
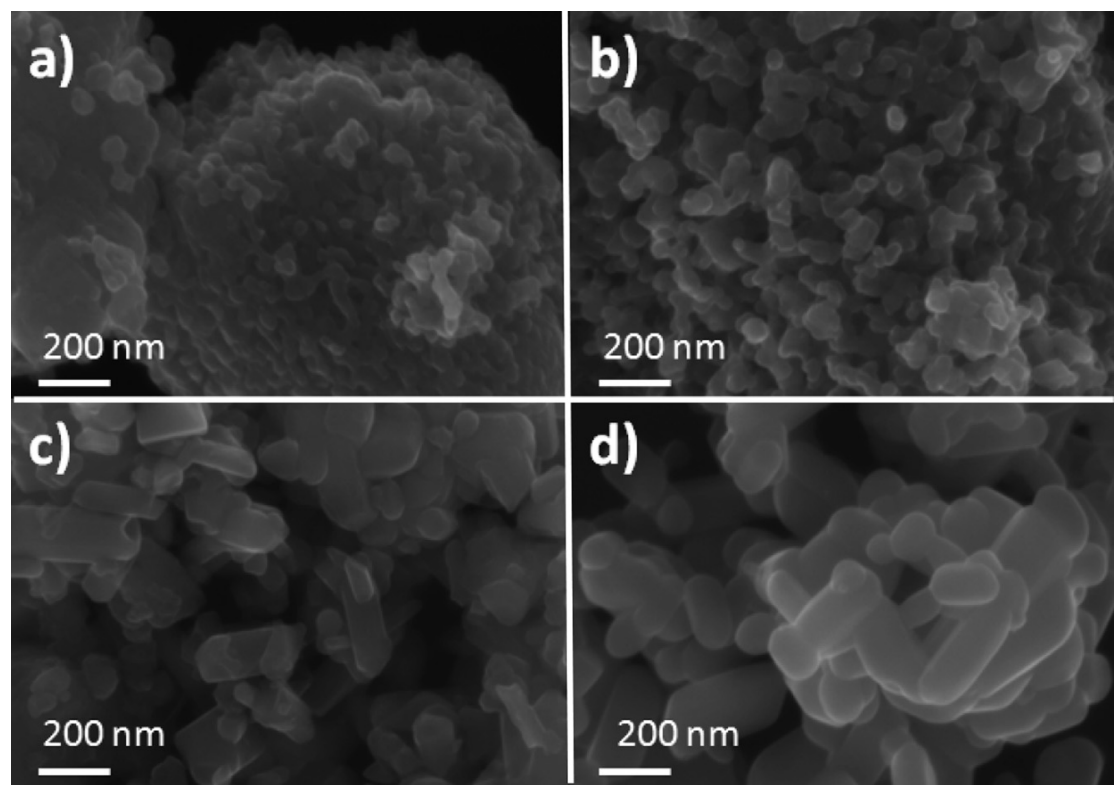

Fig. 7. Micrographs of the $\mathrm{A} 1$ sample at different temperatures: (a) 750 , (b) 800 , (c) 850 and (d) $900{ }^{\circ} \mathrm{C}$. 
3.2. Study of the anatase-rutile transformation and evaluation of the colouring performance of the pigment

Fig. 5 shows the evolution with temperature (from 750 to $1080{ }^{\circ} \mathrm{C}$ ) of the $\mathrm{TiO}_{2}$ polymorphs by Raman spectroscopy. The anatase-rutile transformation occurred at temperatures higher than $850{ }^{\circ} \mathrm{C}$, and it was essentially completed at $1000{ }^{\circ} \mathrm{C}$. The chromatic coordinates of the samples at different temperatures are shown in Table 3 and Fig. 6. A1 sample presents a slight yellow colouration at $180^{\circ} \mathrm{C}$. It is possible to relate the increase of $a^{*}$ and $b^{*}$ chromatic coordinates with the phase transitions between $750{ }^{\circ} \mathrm{C}$ and $1080{ }^{\circ} \mathrm{C}$. The $b^{*}$ coordinate increase from 23 to 36 suggesting
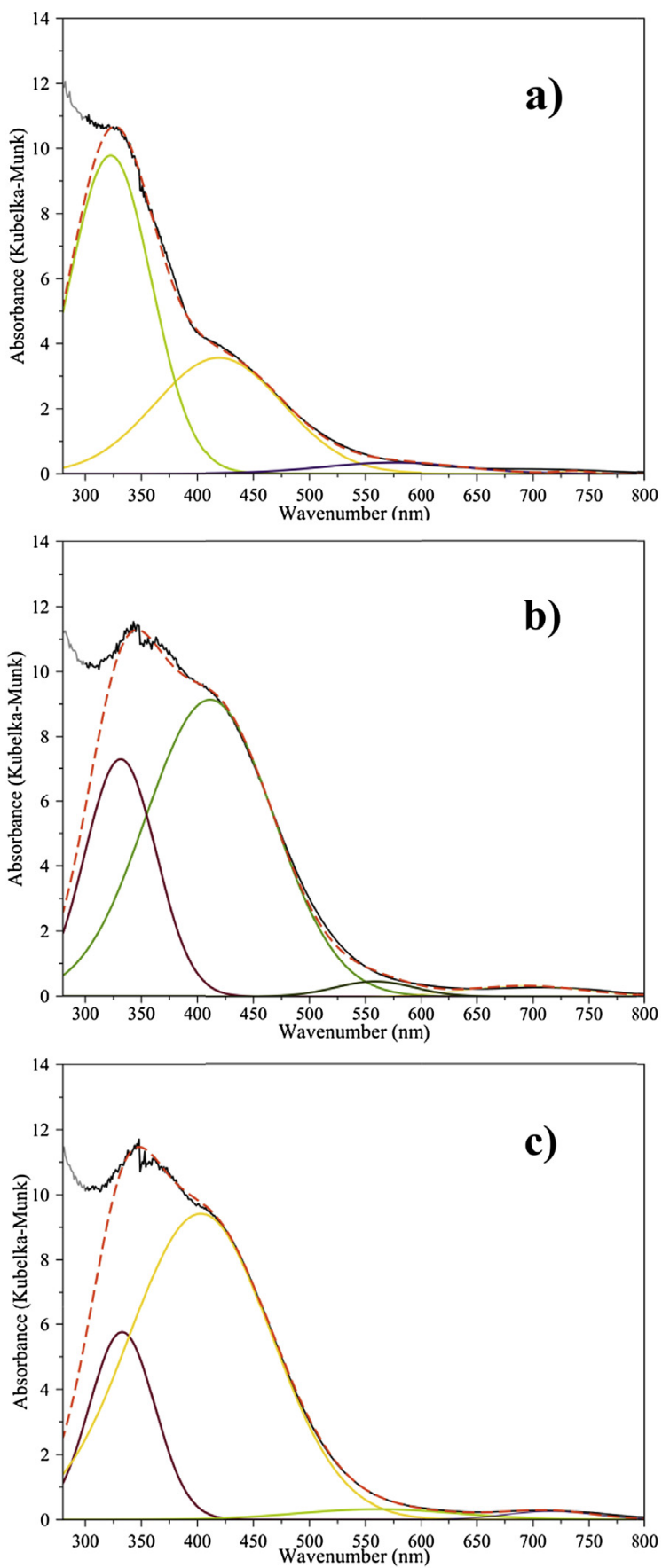

Fig. 8. Diffuse Reflectance spectra for R2 (a), A1 (b) and a commercial ceramic pigment (c) after glazing with deconvolution of optical bands. that there is a relation between the polymorph transformation and the increase of $b^{*}$ coordinate. It could be explain either by the incorporation of the $\mathrm{Cr}$ and $\mathrm{Sb}$ in different environments or, as suggest Matteucci et al. [36,44], because there is a change of the $\mathrm{Sb}$ oxidation state. However, $a^{*}$ coordinate show an approximately linear increase with temperature over the range $750-1000{ }^{\circ} \mathrm{C}$. It is important to highlight a constant $b^{*}$ value in the interval between 900 and $1000{ }^{\circ} \mathrm{C}$. In this range, the main polymorph is rutile but anatase phase exist as minor phase. These results suggest that the phase transformation is still going on at this range of temperatures, probably due to the necessary time for the reconstruction of the structure, from anatase to rutile. After the reconstructive transformation was occurred, and single phase of rutile was obtained at $1080{ }^{\circ} \mathrm{C}$, the value of $b^{*}$ increase significantly.

In order to assess the morphology of the powders during the phase transformation, SEM analysis was made at each temperature. Fig. 7 shows the micrographs for the evolution of the morphology at different temperatures. Shape of the particles was different depending on the polymorph. When anatase is presented, the particles were almost spherical with certain agglomeration, Fig. 7(a) and (b). During the transition, when rutile phase was the majority phase, the particles were prismatic. This fact suggests the elongation of the particles in the polymorphic transition.

\subsection{Stability of the particles as a pigment}

In order to determine the stability of the powders prepared at $180^{\circ} \mathrm{C}$ after glazing, a micropowder/frit mixture was prepared and fired according the cycle set out in the experimental part at the maximum temperature of $1080^{\circ} \mathrm{C}$. The pieces with the glaze were also characterized by UV-Vis and values of the CIELab parameters were obtained. Diffuse reflectance spectra of R2, A1 and a commercial ceramic pigment fired at higher temperatures $\left(>1200{ }^{\circ} \mathrm{C}\right)$ and glazed in the same conditions are presented in Fig. 8 for the 270-800 nm wavelength range. Three main absorption bands are identified in the three spectra after the deconvolution: a broad band located at high energy and centred at $\sim 340 \mathrm{~nm}$ (ultraviolet region) attributed to the bandgap of the rutile [45]; and two bands centred at $\sim 445$ and $560 \mathrm{~nm}$ attributed to the $d-d$ transitions of the $\mathrm{Cr}(\mathrm{III})\left[{ }^{4} \mathrm{~A}_{2}\left({ }^{4} \mathrm{~F}\right) \rightarrow{ }^{4} \mathrm{~T}_{1}\left({ }^{4} \mathrm{~F}\right)\right.$ and ${ }^{4} \mathrm{~A}_{2}\left({ }^{4} \mathrm{~F}\right) \rightarrow{ }^{4} \mathrm{~T}_{2}\left({ }^{4} \mathrm{~F}\right)$, respectively $]$ in an octahedral coordination [39]. Higher absorption of the $d-d$ transition ${ }^{4} \mathrm{~A}_{2}\left({ }^{4} \mathrm{~F}\right) \rightarrow{ }^{4} \mathrm{~T}_{1}\left({ }^{4} \mathrm{~F}\right)$ was observed for the $\mathrm{A} 1$ glazed sample and this intensity was similar to the glazed commercial pigment. Precursor of titanium in solid-solid reactions is usually anatase, which transforms to rutile during the synthesis at high temperatures [38]. The intensity of the absorption bands could be related with the chromatic coordinates shown in Table 4. Good chemical and thermal stability into the frit was obtained and pigments acquired orange colour after glazing with the frit for both samples. Chromatic coordinates were slightly higher for the A1 and similar to those obtained for the commercial ceramic pigment in glaze (similar $\mathrm{a}^{*}$ values). Photographs of the samples before and after mixed with the frit are shown in Fig. 9. In summary, colour of both samples in a standard frit was similar to the colouration of the commercial ceramic pigment obtained at high temperatures. Therefore,

Table 4

CIELab parameters of R2 and A1 samples after glazing (frit). CIELab parameters of a commercial ceramic pigment are included as comparison.

\begin{tabular}{llll}
\hline & \multicolumn{4}{l}{ Powder/Glaze $1080{ }^{\circ} \mathrm{C}$} \\
\cline { 2 - 4 } & $\mathrm{L}^{*}$ & $\mathrm{a}^{*}$ & $\mathrm{~b}^{*}$ \\
\hline R2 & 65 & 12 & 39 \\
$\mathrm{~A} 1$ & 64 & 16 & 41 \\
Commercial $\left(>1200^{\circ} \mathrm{C}\right)$ & 60 & 16 & 47 \\
\hline
\end{tabular}



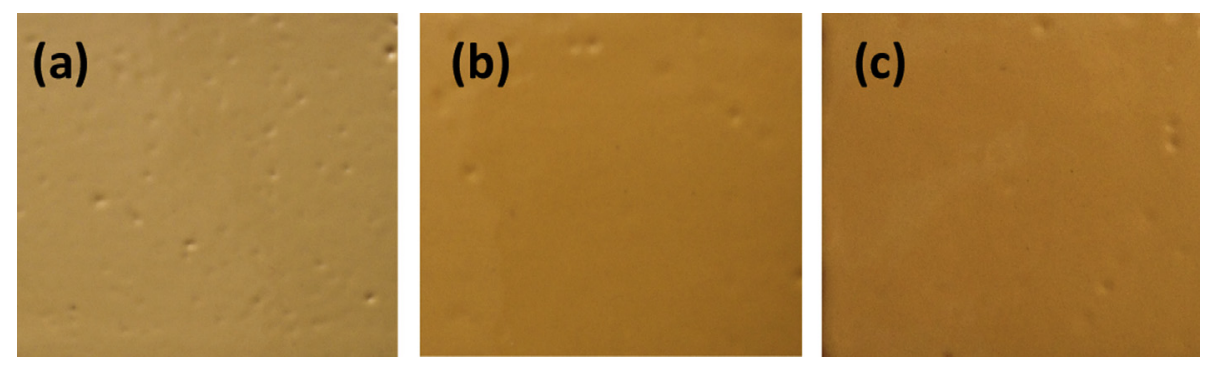

Fig. 9. Photographs of the ceramic tiles for R2 (a), A1 (b) and the commercial pigment (c) after mixed with the frit.

synthesis route has produced orange pigments after glazing, similar of those commercial pigments. It is important to note that these pigments with smaller particle size are stable in glazes, and they developed similar colour of the commercial pigment.

This novel method to synthesize spherical submicron particles at low temperature would presented benefits with respect to the method used in the ceramic industry, which makes use of high temperature calcination. Moreover, secondary phases are normally obtained from traditional ceramic route, not allowing a control of the phase, shape and size. A milling stage must be implemented to adjust the grain size of the pigment due to the high particle size caused by high-temperature calcinations. For these reasons, the novel synthesis method presented in this work to prepare pigments at low temperature would allowed several advantages compared to the traditional solid-solid method, used in the ceramic industry. A possible application of this method in the industry can avoid the milling stages and the calcinations at high temperature.

\section{Conclusions}

Anatase and rutile single phase of a yellow ceramic pigment based on $\mathrm{Cr}, \mathrm{Sb}-\mathrm{TiO}_{2}$ were obtained by microemulsion mediated solvothermal method at $180^{\circ} \mathrm{C}$. The experimental conditions were optimised in order to obtain anatase or rutile phase. These samples prepared at $180{ }^{\circ} \mathrm{C}$ were single phase by XRD, Raman spectroscopy and SEM/EDX. The solvothermal treatment time needed to obtain single phase of anatase or rutile in the $\mathrm{Ti}_{0.97} \mathrm{Cr}_{0.015} \mathrm{Sb}_{0.015} \mathrm{O}_{2}$ solid solution was $17 \mathrm{~h}$ and $24 \mathrm{~h}$, respectively. Spherical particles were formed in both cases with an average particle size of $600 \mathrm{~nm}$ and a $\zeta$-potential value around $-57 \mathrm{mV}$. The polymorphic transition temperature and the changes of the morphology that this transformation involves were determined by Raman and SEM. The anatase-rutile transformation occurred at temperatures higher than $850{ }^{\circ} \mathrm{C}$, and it was essentially completed at $1000^{\circ} \mathrm{C}$. Elongation of the particles in the polymorphic transition was observed. There is also a correlation between the polymorph transformation and the increase of the chromatic coordinates measured by UV-Vis, leading to a huge orange colour pigment. Samples have a good chemical and thermal stabilization into the frit, presenting similar chromatic coordinates to those of the commercial ceramic pigment obtained at high temperatures, especially when the anatase solid solution at $180{ }^{\circ} \mathrm{C}$ was the starting powder. Orange colour was kept after the application on glazes. Therefore, size, shape, colour and electrostatic stability of these particles, prepared by microemulsion-mediated solvothermal route, make it a potential candidate for orange ceramic pigment to be incorporated in the inkjet technology.

\section{Acknowledgements}

We thank the "Universitat Jaume I"-project No. P1 1B2013-65 (MJ, MD, HB, EC) and the "INCTMN"-2008/57872-1, "FAPESP"-2013/
11144-3; 2013/07296-2, and “CNPq" 573636/2008-7 (TRM and EL) for financial support. M.J. also thanks this project for a fellowship. We also thank to Juan Carlos Gallard (Ferro Spain S.A.) for the initial ideas.

\section{References}

[1] Pfaff G, Reynders P. Angle-dependent optical effects deriving from submicron structures of gilms and pigments. Chem Rev 1999;99:1963-82.

[2] Salvador A, Pascual-Martí MC, Adell JR, Requeni A, March JG. Analytical methodologies for atomic spectrometric determination of metallic oxides in UV sunscreen creams. J Pharm Biomed Anal 2000;22:301-6.

[3] Zallen R, Moret MP. The optical absorption edge of brookite $\mathrm{TiO}_{2}$. Solid State Commun 2006;137:154-7.

[4] Braun JH, Baidins A, Marganski RE. $\mathrm{TiO}_{2}$ pigment technology: a review. Prog Org Coatings Prog Org Coat 1992:20:105-38.

[5] Yuan S, Chen W, Hu S. Fabrication of $\mathrm{TiO}_{2}$ nanoparticles/surfactant polymer complex film on glassy carbon electrode and its application to sensing trace dopamine. Mater Sci Eng C 2005;25:479-85.

[6] Mo S, Ching Y. Electronic and optical properties of three phases of titanium dioxide: rutile, anatase, and brookite. Phys Rev 1995;51:23-32.

[7] Hanaor DH, Sorrell CC. Review of the anatase to rutile phase transformation. J Mater Sci 2010;46:855-74.

[8] Braginsky L, Shklover V. Light absorption in $\mathrm{TiO}_{2}$ nanoparticles. Eur Phys JD 1999;630:627-30.

[9] Beydoun D, Amal R. Implications of heat treatment on the properties of a magnetic iron oxide-titanium dioxide photocatalyst. Mater Sci Eng B 2002:94:71-81.

[10] Chen X, Mao SS. Titanium dioxide nanomaterials: synthesis, properties, modifications, and applications. Chem Rev 2007;107:2891-959.

[11] Reidy DJ, Holmes JD, Morris MA. The critical size mechanism for the anatase to rutile transformation in $\mathrm{TiO}_{2}$ and doped-TiO 2 .J Eur Ceram Soc 2006;26:1527-34.

[12] Yin S, Hasegawa H, Maeda D, Ishitsuka M, Sato T. Synthesis of visible-light-active nanosize rutile titania photocatalyst by low temperature dissolution-reprecipitation process. J Photochem Photobiol A Chem 2004:163:1-8.

[13] Li G, Boerio-Goates J, Woodfield BF, Li L. Evidence of linear lattice expansion and covalency enhancement in rutile $\mathrm{TiO}_{2}$ nanocrystals. Appl Phys Lett 2004;85:2059-61.

[14] Gardini D, Dondi M, Costa AL, Matteucci F, Blosi M, Galassi C, et al. Nano-sized ceramics inks for drop-on-demand ink-jet printing in quadrichromy. J Nanosci Nanotechnol 2008;8:1979-88.

[15] Dondi M, Blosi M, Gardini D, Zanelli C. Ceramic pigments for digital decoration inks: an overview. Castellón: Qualicer; 2012. p. 1-11.

[16] Kosmala A, Wright R, Zhang Q Kirby P. Synthesis of silver nano particles and fabrication of aqueous Ag inks for inkjet printing. Mater Chem Phys 2011;129: 1075-80.

[17] Feldmann C, Jungk HO. Polyol-mediated preparation of nanoscale oxide particles. Angew Chem Int Ed 2001;40:359-62.

[18] Baldi G, Bitossi M, Barzanti A. US patent 2008; 7316741 B2.

[19] Cavalcante PMT, Dondi M, Guarini G, Raimondo M, Baldi G. Colour performance of ceramic nano-pigments. Dye Pigment 2009;80:226-32.

20] Zhong Z, Ang TP, Luo J, Gan H, Gedanken A. Synthesis of one-dimensional and porous $\mathrm{TiO}_{2}$ nanostructures by controlled hydrolysis of titanium alkoxide via coupling with an esterification reaction. Chem Mater 2005;17:6814-8.

[21] Song KC, Pratsinis SE. Control of phase and pore structure of titania powders using $\mathrm{HCl}$ and $\mathrm{NH}_{4} \mathrm{OH}$ catalysts. J Am Ceram Soc 2001;84:92-8.

[22] Tayade RJ, Kulkarni RG, Jasra RV. Photocatalytic degradation of aqueous nitrobenzene by nanocrystalline $\mathrm{TiO}_{2}$. Ind Eng Chem Res 2006;45:922-7.

[23] Murakami Y, Matsumoto T, Takasu Y. Salt catalysts containing basic anions and acidic cations for the sol-gel process of titanium alkoxide: controlling the kinetics and dimensionality of the resultant titanium oxide. J Phys Rev B 1999;103:1836-40.

[24] Cheng H, Ma J, Zhao Z, Qi L. Hydrothermal preparation of uniform nanosize rutile and anatase particles. Chem Mater 1995;7:663-71.

[25] Li G, Gray KA. Preparation of mixed-phase titanium dioxide nanocomposites via solvothermal processing. Chem Mater 2007;19:1143-6.

[26] Lal M, Chhabra V. Preparation and characterization of ultrafine $\mathrm{TiO}_{2}$ particles in reverse micelles by hydrolysis of titanium di-ethylhexyl sulfosuccinate. J Mater Res 1998;13:1249-54. 
[27] Andersson M, Lars O. Preparation of nanosize anatase and rutile $\mathrm{TiO}_{2}$ by hydrothermal treatment of microemulsions and their activity for photocatalytic wet oxidation of Phenol. J Phys Chem B 2002;106:10674-9.

[28] Deorsola FA, Vallauri D. Study of the process parameters in the synthesis of $\mathrm{TiO}_{2}$ nanospheres through reactive microemulsion precipitation. Powder Technol 2009;190:304-9.

[29] Cao M, Wu X, He X, Hu C. Microemulsion-mediated solvothermal synthesis of $\mathrm{SrCO}_{3}$ nanostructures. Langmuir 2005;21:6093-6.

[30] Cao M, Wang Y, Guo C, Oi Y, Hu C. Preparation of ultrahigh-aspect-ratio hydroxyapatite nanofibers in reverse micelles under hydrothermal conditions. Langmuir 2004;20:4784-6.

[31] Wu M, Long J, Huang A, Luo Y. Microemulsion-mediated hydrothermal synthesis and characterization of nanosize rutile and anatase particles. Langmuir 1999; 15:8822-5.

[32] Lu CH, Wu WH, Kale RB. Microemulsion-mediated hydrothermal synthesis of photocatalytic $\mathrm{TiO}_{2}$ powders. J Hazard Mater 2008;154:649-54.

[33] Shen X, Zhang J, Tian B. Microemulsion-mediated solvothermal synthesis and photocatalytic properties of crystalline titania with controllable phases of anatase and rutile. J Hazard Mater 2011;192:651-7.

[34] Aruna ST, Tirosh S, Zaban A. Nanosize rutile titania particle synthesis via a hydrothermal method without mineralizers. J Mater Chem 2000;10:2388-91.

[35] Sōmiya S, Roy R. Hydrothermal synthesis of fine oxide powders. Bull Mater Sc 2000:23:453-60.

[36] Matteucci F, Cruciani G, Dondi M, Raimondo M. The role of counterions (Mo, $\mathrm{Nb}, \mathrm{Sb}, \mathrm{W})$ in $\mathrm{Cr}-$, $\mathrm{Mn}-$-, Ni- and $\mathrm{V}$-doped rutile ceramic pigments. Ceram Int 2006;32:385-92.
[37] Batzill M, Morales EH, Diebold U. Influence of nitrogen doping on the defect formation and surface properties of $\mathrm{TiO}_{2}$ rutile and anatase. Phys Rev Lett 2006;96:026103. 1-4.

[38] Gargori C, Cerro S, Galindo R, Monrós G. In situ synthesis of orange rutile ceramic pigments by non-conventional methods. Ceram Int 2010;36:23-31.

[39] Cruciani G, Dalconi MC, Dondi M, Meneghini C, Matteucci F, Barzanti A, et al. Temperature-resolved synchrotron X-ray diffraction of nanocrystalline titania in solvent: the effect of $\mathrm{Cr}-\mathrm{Sb}$ and $\mathrm{V}-\mathrm{Sb}$ doping. J Nanopart Res 2010;13: 711-9.

[40] Marfunin S. Physics of minerals and inorganic materials. Berlin, Heildelberg, New York: Springer; 1979.

[41] Chang H, Huang PJ. Thermo-raman studies on anatase and rutile. J Raman Spectrosc 1998;29:97-102.

[42] Sammelselg V, Tarre A, Lu J, Aarik J, Niilisk A, Uustare T, et al. Structural characterization of $\mathrm{TiO}_{2}-\mathrm{Cr}_{2} \mathrm{O}_{3}$ nanolaminates grown by atomic layer deposition. Surf Coatings Technol 2010;204:2015-8.

[43] Wakeman RJ, Marchant JQ. The influence of $\mathrm{pH}$ and temperature on the rheology and stability of aqueous titanium dioxide dispersions. Chem Eng J 1997;67:97-102.

[44] Dondi M, Cruciani G, Guarini G, Matteucci F, Raimondo M. The role of counterions (Mo, Nb, Sb, W) in $\mathrm{Cr}-$-, Mn-, Ni- and V-doped rutile ceramic pigments. Ceram Int 2006;32:393-405.

[45] Yan J, Wu G, Guan N, Li L, Li Z, Cao X. Understanding the effect of surface/bulk defects on the photocatalytic activity of $\mathrm{TiO}_{2}$ : anatase versus rutile. Phys Chem Chem Phys 2013;15:10978-88. 

